\title{
OBSERVATION OF MUON NEUTRINO CHARGED CURRENT EVENTS IN AN OFF-AXIS HORN-FOCUSED NEUTRINO BEAM USING THE NO $\nu$ A PROTOTYPE DETECTOR
}

By

\author{
Enrique Arrieta Díaz
}

\section{A DISSERTATION}

\author{
Submitted to \\ Michigan State University \\ in partial fulfillment of the requirements \\ for the degree of \\ Physics - Doctor of Philosophy
}

2014 


\title{
ABSTRACT \\ OBSERVATION OF MUON NEUTRINO CHARGED CURRENT EVENTS IN AN OFF-AXIS HORN-FOCUSED NEUTRINO BEAM USING THE NO $\nu$ A PROTOTYPE DETECTOR
}

\author{
By
}

\section{Enrique Arrieta Díaz}

The $\mathrm{NO} \nu \mathrm{A}$ is a long base-line neutrino oscillation experiment. It will study the oscillations between muon and electron neutrinos through the Earth. NO $\nu \mathrm{A}$ consists of two detectors separated by $810 \mathrm{~km}$. Each detector will measure the electron neutrino content of the neutrino (NuMI) beam. Differences between the measurements will reveal details about the oscillation channel. The $\mathrm{NO} \nu \mathrm{A}$ collaboration built a prototype detector on the surface at Fermilab in order to develop calibration, simulation, and reconstruction tools, using real data. This 220 ton detector is $110 \mathrm{mrad}$ off the NuMI beam axis. This off-axis location allows the observation of neutrino interactions with energies around $2 \mathrm{GeV}$, where neutrinos come predominantly from charged kaon decays. During the period between October 2011 and April 2012, the prototype detector collected neutrino data from $1.67 \times 10^{20}$ protons on target delivered by the NuMI beam. This analysis selected a number of candidate charged current muon neutrino events from the prototype data, which is $30 \%$ lower than predicted by the $\mathrm{NO} \nu \mathrm{A}$ Monte Carlo simulation. The analysis suggests that the discrepancy comes from an over estimation of the neutrino flux in the Monte Carlo simulation, and in particular, from neutrinos generated in charged kaon decays. The ratio of measured divided by the sim-

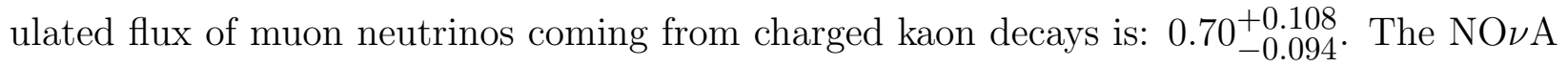
collaboration may use the findings of this analysis to introduce a more accurate prediction of the neutrino flux produced by the NuMI beam in future Monte Carlo simulations. 
To my abuelo, Andrés, Quique and Rochi.

The precious components of my high intensity beam of unconditional love. 


\section{ACKNOWLEDGMENTS}

I can not pinpoint a moment in my life as the time when I decided to be a scientist. However, I can assure that my decision consolidated during my third year of elementary school in the classroom of C. Cabezas. He impacted my life forever, and my incommensurable gratitude accompanies him now in the stars, right where he belongs. Soon after I learned from M. Rios that a successful man can only live under the strict regime of impeccable moral standards, and I have tried to follow his teachings to the best of my capacities. During my junior and senior years of high school I sat in the classroom of C. Martínez where he elegantly provided most of the knowledge in physics that I bear today. It was at this time when I told F. Casas that if by 2007 I was not a rey de reyes in Valledupar, he could be certain that I would then become a Ph.D. in physics. Thanks to him I was introduced to the books of Carl Sagan that structured my scientific thinking. Thanks to all my teachers at Abraham Lincoln school, and to my professors at FIT, Los Andes, and MSU for their wonderful lessons.

The long road that brought me to my present had many gratifying moments, and countless steep slopes and deep troughs. This road, that resembled that to Santa Ana during heavy winter times, felt very smooth thanks to the love and support of those members of my families Aarón, Arrieta, Díaz, and Hamburger, that always believed in me. I never tried to make them feel proud, however I have been working extremely hard not to make them feel ashamed. My moments of happiness were doubled, and my periods of sadness were halved, when I had the chance to share them with my siblings from the $16^{\text {th }}$ street and my fellow Wachakeros. When the wipers did not work due to the mud covering the windshield, only the GPS guidance of E. M. Aarón helped me to reach safe shelter. My personal oracle, A. Op den Bosch, kept me safe and well advised. An especial gratefulness remains in my heart 
for the members of the Solano family that cared for me like one of their own. Many friends have their two cents and their grains of sand deposited in the safe of my heart. I can not name them all, but I do treasure all their support throughout the years.

I have felt like Achilles racing the tortoise many times. Nonetheless, there is always someone out there to remind me that only a fool can not defeat a tortoise at a speed race, and that I am not a fool. M. C. Jácome and M. Nowakowski made this very clear to me. However, it is only with the help of extraordinary persons that extraordinary goals are accomplished. I am lucky enough to be surrounded by extraordinary individuals like S. D. Mahanti, J. Paley, and S. Pratt who supported me, beyond imagination, when I most needed them. There is no word in human languages to express the infinite gratitude that I have for them. My friendships with J. Clifford, E. Kessler, T. Olson, and L. C. Rodríguez made of my years at MSU a wonderful time.

The quest in $\mathrm{NO} \nu \mathrm{A}$ started with the Fellowship of the Fiber, and my companions M. Nila, R. Richards, and D. Shooltz. Days of darkness were illuminated by their amazing personalities. Then I arrived to the land of Fermilab where I found the invaluable support of M. Betancourt. After meeting her the locomotive became unstoppable. M. Sánchez and P. Vahle provided endless fuel for my endeavor. My analysis was only possible with the outstanding patronage of C. Backhouse, M. Baird, M. Frank, R. Hatcher, N. Mayer, M. Messier, M. Muether, E. Niner, R. Patterson, G. Pawloski, N. Raddatz, P. Shanahan, and Z. Wang. A. Sousa greatly contributed to my success with his knowledge about the divine and human. Indubitably the entire $\mathrm{NO} \nu \mathrm{A}$ collaboration made it happen. The last portion of my journey, at Argonne Lab, was enlightened by M. Goodman, and S. Magill, R. Talaga, and the members of the neutrino group. They made me feel like home. In particular, J. A. Sepúlveda showed me the true meaning of humbleness and kindness. I will be forever thankful to D. 
Barratt, E. Johnson, B. Wenzlick, and R. Young. Their magic always simplified my life.

Mission accomplished! Mabel can now host the party, and Manolo can prepare the hug and the entertainment. Leandro made El Cardón Guajiro, and Diomedes sang it for me. The great days are straight ahead because I am, indeed, made for great things. Thank you!

"Hay soledades que duelen mucho, hay un silencio para pensar, hoy quiero luces para que alumbren lo que me falta por caminar. Soy andariego de los caminos, con palabritas quiero decir, así es mi vida siempre cambiando, un día muy triste y un día feliz. Solo le pido a la vida, que me dé felicidad, y que mi conciencia duerma siempre tranquila. Quiero sentir el aprecio de los amigos, vivir feliz en mi Valle, no pido más. No pido más, no quiero ser golondrina que errante va. No pido más, hoy quiero luces que alumbren mi oscuridad." Gustavo Gutiérrez Cabello 


\section{TABLE OF CONTENTS}

LIST OF TABLES ....................... . . . . .

LIST OF FIGURES . . . . . . . . . . . . . . . . . . . . xi

Chapter 1 Introduction f...................... 1

1.1 Brief History Of Neutrinos . . . . . . . . . . . . . . . . . . . . 2

1.2 Motivations For The $\mathrm{NO} \nu \mathrm{A}$ Experiment . . . . . . . . . . . . . . . 3

1.3 Outline . . . . . . . . . . . . . . . . . . . . . 7

Chapter 2 Theoretical Framework for Neutrinos . . . . . . . . . . . . 9

2.1 Neutrinos In Weak Interactions . . . . . . . . . . . . . . . . . 9

2.1.1 Charged Current Interactions . . . . . . . . . . . . . . . . . . 10

2.1.1.1 Quasi-elastic Scattering . . . . . . . . . . . . . 11

2.1.1.2 Resonant Pion Production . . . . . . . . . . . . . . . 14

2.1.1.3 Deep Inelastic Scattering . . . . . . . . . . . . . . . . . 16

2.1.1.4 Inclusive Cross Section Measurements . . . . . . . . . . . 17

2.1 .2 Neutral Current Interactions . . . . . . . . . . . . . . . . . . 20

2.2 Neutrino Masses . . . . . . . . . . . . . . . . . . . . . . . . . 23

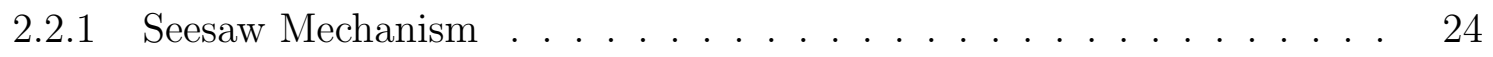

2.3 Neutrino Oscillations . . . . . . . . . . . . . . . . . . . . . . . . 26

Chapter 3 NuMI Beam Overview . . . . . . . . . . . . . . . . . . . 34

3.1 Primary Beam System . . . . . . . . . . . . . . . . . . . . . . . . . . . . . . . . 38

3.2 Secondary Beam System . . . . . . . . . . . . . . . . . . . . . 39

3.3 NuMI Beam Composition And Energy Spectrum At $110 \mathrm{mrad}$. . . . . . . . 41

Chapter 4 The NO $\nu$ Prototype Detector . . . . . . . . . . . . . . 47

4.1 Experiment Overview . . . . . . . . . . . . . . . . . . . 47

4.2 The Near Detector On The Surface . . . . . . . . . . . . . . . . 51

4.2 .1 Liquid Scintillator . . . . . . . . . . . . . . . . . . . . . 56

4.2.2 Wavelength Shifting Fiber . . . . . . . . . . . . . . . . 56

4.2.3 Avalanche Photodiodes . . . . . . . . . . . . . . . . . . 58

4.2 .4 Front-End Boards . . . . . . . . . . . . . . . . . . . 60

4.2.5 Data Acquisition System . . . . . . . . . . . . . . . . 61

4.2.6 Performance And Calibration Of The Prototype Detector . . . . . . . 63

$4.3 \mathrm{NO} \nu \mathrm{A}$ Software . . . . . . . . . . . . . . . . . . . . . . . . . . . . . . . . . . 69

4.3.1 Monte Carlo Simulation . . . . . . . . . . . . . . . . 70

4.3.2 Reconstruction Tools . . . . . . . . . . . . . . . . . 71

4.3.2.1 The Slicer . . . . . . . . . . . . . . . . 71 
4.3.2.2 Track Reconstruction . . . . . . . . . . . . . . . . . . 73

4.4 Data Quality . . . . . . . . . . . . . . . . . . . . . . 81

4.5 Cosmic Rays . . . . . . . . . . . . . . . . . . . . . . . . 8 86

Chapter 5 Event Selection . . . . . . . . . . . . . . . . . . . . 93

5.1 Charged And Neutral Current Neutrino Interactions . . . . . . . . . . . . . . 95

5.2 Event Containment Criteria . . . . . . . . . . . . . . . . . . . . . . . . . 99

5.3 Charged Current Event Selection _. . . . . . . . . . . . . . . 103

5.3 .1 Longest Track Length Cut . . . . . . . . . . . . . . . . . . . 103

5.3 .2 Cosmic Ray Cuts . . . . . . . . . . . . . . . . . . . . . . . . . 105

5.3 .3 Minimum Ionizing Particle Cut . . . . . . . . . . . . . . . . . 109

Chapter 6 Event Energy Reconstruction . . . . . . . . . . . . . . . . 115

6.1 Muon Energy Estimation . . . . . . . . . . . . . . . . . . . . . . . 115

6.2 Hadronic Energy Estimation . . . . . . . . . . . . . . . . . . . . . 121

6.3 Quasi-elastic And Non-quasi-elastic Classification . . . . . . . . . . . . 125

6.4 Neutrino Energy Estimation . . . . . . . . . . . . . . . . . . . . 130

Chapter 7 Systematic Uncertainties . . . . . . . . . . . . . . . . . . . . . 137

7.1 Energy Estimation Uncertainty . . . . . . . . . . . . . . . . . . . 138

7.2 Prototype Detector's Channels Configuration Uncertainty . . . . . . . . . . . 141

7.3 GENIE Cross Sections And Final State Physics Uncertainty . . . . . . . . 142

7.4 Unfolding Systematic Uncertainty . . . . . . . . . . . . . . . . 147

7.5 Total Systematic Uncertainty . . . . . . . . . . . . . . . . . . 148

Chapter 8 Analysis . . . . . . . . . . . . . . . . . . . . . . 152

8.1 Data And Monte Carlo Simulation Comparison . . . . . . . . . . . . . 152

8.2 Results . . . . . . . . . . . . . . . . . . . . . . . 171

Chapter 9 Discussion And Final Remarks . . . . . . . . . . . . 176

9.1 Flux Calculation . . . . . . . . . . . . . . . . . . . . . 176

9.1.1 Number Of Atoms In The Target Region . . . . . . . . . . . . 180

9.1.2 Monte Carlo Cross Sections . . . . . . . . . . . . . . . . . . . . . 182

9.1.3 Total Reconstruction Efficiency . . . . . . . . . . . . . . . . . . 184

9.1.4 $\nu_{\mu}+\bar{\nu}_{\mu}$ Flux Coming From Charged Kaon Decays . . . . . . . . . 185

9.2 Inclusive $\nu_{\mu}$ Charged Current Cross Section Calculation . . . . . . . . . . 189

9.3 Final Remarks . . . . . . . . . . . . . . . . . . . . . . . . . . . 195

APPENDICES . . . . . . . . . . . . . . . . . . . . . 197

Appendix A Mass Terms In The Weak Interaction . . . . . . . . . . . . 197

Appendix B $\mathrm{NO} \nu \mathrm{A}$ Kalman Tracker . . . . . . . . . . . . . . . . . . . . . 204

Appendix C Unfolding Algorithm, TSVDUnfold . . . . . . . . . . . . 209

Appendix D List Of Muon Neutrino Charged Current Candidate Events . 220 


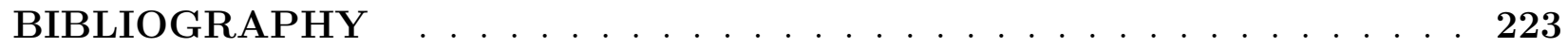




\section{LIST OF TABLES}

Table 2.1 Neutrino Oscillation Parameters. . . . . . . . . . . . . . . 32

Table 4.1 Kalman Tracker Performance. . . . . . . . . . . . . . . . . . . . . 74

Table 5.1 Event Selection Performance. . . . . . . . . . . . . . 114

Table 7.1 GENIE Parameters. . . . . . . . . . . . . . . . . . . . . . 145

Table 8.1 Event Selection Cuts. . . . . . . . . . . . . . . 159

Table 8.2 Quasi-elastic And Non-quasi-elastic Classification Performance. . . . . . . . . . . . . . . . . . 159

Table 8.3 Candidate Events With Background. . . . . . . . . . . . . . 173

Table 8.4 Number Of Candidate Events. . . . . . . . . . . . . . . . . 174

Table 8.5 Neutral Current Background. . . . . . . . . . . . . . . . . . 174

Table 8.6 Electron Neutrino Background. . . . . . . . . . . . . . . . . 175

Table 9.1 Background To Neutrinos From Kaon Decays. . . . . . . . . . . 178

Table 9.2 Candidate Events From Kaon Decays. . . . . . . . . . . . . . . . 179

Table 9.3 Chemical Composition Of The Prototype Detector. . . . . . . 181

Table D.1 Quasi-elastic Candidate Events. . . . . . . . . . . . . 220

Table D.2 Non-quasi-elastic Candidate Events, I . . . . . . . . . . . . . . . 221

Table D.3 Non-quasi-elastic Candidate Events, II . . . . . . . . . . . . . . 222 


\section{LIST OF FIGURES}

Figure 2.1 Weak Interaction Charged Current Representation Of Neutrino-nucleon Scattering. . . . . . . . . . . . . . . . . . . . 11

Figure 2.2 Experimental Results On Muon Neutrino Quasi-elastic Cross Sections. . . . . . . . . . . . . . . . . . 14

Figure 2.3 Theoretical And Experimental Results On Muon Neutrino Resonance Cross Sections. . . . . . . . . . . . . . . . 15

Figure 2.4 Experimental Results On The Total Inclusive Muon Neutrino Charged Current Cross Sections. . . . . . . . . . 17

Figure 2.5 Brookhaven National Laboratory Muon Neutrino Inclusive Cross Sections. . . . . . . . . . . . . . . . . . 18

Figure 2.6 SciBooNE Muon Neutrino Inclusive Cross Sections. . . . . 19

Figure 2.7 Weak Interaction Neutral Current Representation Of Neutrino-nucleon Scattering. . . . . . . . . . . . . . . . . . . . 21

Figure 2.8 MiniBooNE Neutral Current Differential Cross Section. . . 22

Figure 2.9 Neutrino Mass Hierarchy. . . . . . . . . . . . . . . . . . . . . . . 33

Figure $3.1 \quad$ Fermilab Accelerator Campus. . . . . . . . . . . . . . . . 34

Figure $3.2 \quad$ NuMI Beam Trajectory. . . . . . . . . . . . . . . . . . . . . 34

Figure $3.3 \quad$ NuMI Beam Energy Spectrum. . . . . . . . . . . . . . . . . 35

Figure $3.4 \quad$ Location of The NO $\nu$ A And MINOS Far Detectors. . . . 36

Figure $3.5 \quad$ Schematics Of The NuMI Complex. . . . . . . . . . . . . 37

Figure $3.6 \quad$ NuMI Beam Schematics. . . . . . . . . . . . . . . . . . . . . 37

Figure $3.7 \quad$ NuMI Target Drawing. . . . . . . . . . . . . . . . . . . . . 39

Figure 3.8 Beam And Electric Current Directions Through The NuMI Horns. . . . . . . . . . . . . . . . . . . . . 40 
Figure 3.9 Meson Transverse And Longitudinal Momenta. . . . . . . . . 43

Figure 3.10 Neutrino Energy Distribution Discriminated By The Mother Particle Of The Neutrino. . . . . . . . . . . . . . . . . 44

Figure 3.11 Neutrino Energy Distribution Discriminated By The Neu-

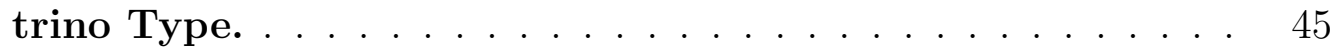

Figure 3.12 Ratio Of Neutrinos From Charged Kaon Decays Over Neutrinos From Charged Pion Decays. . . . . . . . . . . . . . 46

Figure $4.1 \quad$ Electron Neutrino Bi-probability Plot. . . . . . . . . . . . . 48

Figure 4.2 Drawing Of The $\mathbf{N O} \nu$ A Detectors. . . . . . . . . . 50

Figure 4.3 Angle Of Neutrinos With Respect To The NuMI Beam. . . 51

Figure 4.4 Meson Momentum And Neutrino Energy As A Function Of The NuMI Angle. . . . . . . . . . . . . . . . . . 52

Figure $4.5 \quad$ Drawing Of The $\mathrm{NO} \nu \mathrm{A}$ Prototype Detector. . . . . . . . 53

Figure $4.6 \quad \mathrm{NO} \nu \mathrm{A}$ Extrusions And Cells Sample. . . . . . . . . . . . 54

Figure 4.7 Side View Of The $\mathrm{NO} \nu \mathrm{A}$ Prototype Detector. . . . . . . . 54

Figure 4.8 Emission Spectra Of The Wavelength Shifting Fiber. . . . 57

Figure 4.9 Wavelength Shifting Fiber Transverse Area. . . . . . . . . 58

Figure 4.10 Avalanche Photodiode. . . . . . . . . . . . . . . . . . 59

Figure 4.11 Schematic Overview Of The Data Acquisition System. . . . 62

Figure 4.12 Event Display Of The NO $\nu$ A Prototype Detector. . . . . . . 64

Figure 4.13 Cosmic Data Of The NO $\nu$ A Prototype Detector: Light Output vs. Distance. . . . . . . . . . . . . . . . 65

Figure 4.14 Energy Calibration Of The NO $\nu$ A Prototype Detector. . . . 66

Figure 4.15 Energy Conversion Factors Of The NO $\nu$ A Prototype Detec-

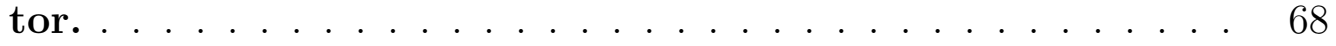

Figure 4.16 Light Level Over Time On The NO $\nu$ A Prototype Detector. 69 
Figure 4.17 Sample Cosmic Ray Event For Slicer Performance. . . . . . . 72

Figure 4.18 Event Vertex Resolution. . . . . . . . . . . . . . . . 75

Figure 4.19 Longest Track Endpoint Resolution. . . . . . . . . . . . . . 76

Figure 4.20 Sample Event Of Tracking Algorithm Corrections. Case 1. . 77

Figure 4.21 Sample Events Of Tracking Algorithm Corrections. Cases 2 To 4. . . . . . . . . . . . . . . . . . . . . . . . . . . . . . . . 79

Figure 4.22 Sample Event Of Tracking Algorithm Corrections. . . . . . . 80

Figure 4.23 Number Of Active Channels In The NO $\nu$ A Prototype Detector. . . . . . . . . . . . . . . . . . 81

Figure 4.24 Number Of Neutrino Candidates As A Function Of The Protons On Target In The NO $\nu$ A Prototype Detector. . . . . . . 83

Figure 4.25 Time Of Event Slice In The Data Of The NO $\nu$ A Prototype Detector. . . . . . . . . . . . . . . 83

Figure 4.26 Sample Trigger Time Window With A Selected Neutrino Candidate. . . . . . . . . . . . . . . . . 84

Figure 4.27 Time Of Slices In The Data Of The Prototype Detector After Event Selection. . . . . . . . . . . . . . 86

Figure 4.28 Sample Trigger Time Window With Cosmic Ray Events. . . 87

Figure 4.29 Sample Cosmic Ray Event. . . . . . . . . . . . . . . . . . . . 88

Figure 4.30 Beam To NO $\nu$ A Prototype Detector Coordinate Transformation Illustration. . . . . . . . . . . . . . . . . . . . . . . 89

Figure 4.31 The Direction Of Neutrinos From The NuMI Beam In The Prototype Detector. . . . . . . . . . . . . . . . . 91

Figure 4.32 Longest Track Angular Distributions For Data And Monte Carlo Simulation. . . . . . . . . . . . . . . . . . . . . . . . . 92

Figure $5.1 \quad$ Single Particle Events. . . . . . . . . . . . . . . . . . . 94

Figure $5.2 \quad$ Sample Events For Neutral Current Background. . . . . . . . 95 
Figure 5.3 Neutrino Energy Distributions From The NO $\nu$ A Prototype

Detector. . . . . . . . . . . . . . . . 96

Figure 5.4 Monte Carlo Prediction Of The Ratio Of Charged Current

To Neutral Current Neutrino Interactions. . . . . . . . . . . 97

Figure 5.5 Fraction of Reconstructed Charged Current Events. . . . . 97

Figure $5.6 \quad$ Ratio Of Reconstructed Over Simulated Events. . . . . . . . 98

Figure $5.7 \quad$ Vertex Region Of The $\mathrm{NO} \nu \mathrm{A}$ Prototype Detector. . . . . . 100

Figure 5.8 Containment Region Of The $\mathrm{NO} \nu \mathrm{A}$ Prototype Detector. . . 101

Figure 5.9 Monte Carlo Simulated Longest Track Length Distributions

For Muons and Non-muons. . . . . . . . . . . . . . . . 103

Figure 5.10 Monte Carlo Simulation Of The Longest Track Length Distributions. . . . . . . . . . . . . . . . . . . 104

Figure 5.11 Cosmic Rays Rejection Zone By The Cut In $\cos \theta_{Y}$. . . . 106

Figure 5.12 Cosmic Rays Rejection Zone By The Cut In $\cos \theta_{\text {NuMI }} \ldots 108$

Figure $5.13 \quad \cos \theta_{Y}$ vs. $\cos \theta_{\mathbf{N u M I}} \cdot \ldots \ldots \ldots \ldots \ldots$

Figure 5.14 Cosmic Track Mean $\boldsymbol{d} \boldsymbol{E} / \boldsymbol{d} \boldsymbol{X} \ldots \ldots \ldots$

Figure 5.15 Minimum Ionizing Particle Fraction. . . . . . . . . . . . . 110

Figure 5.16 Longest Track Particle Identity. . . . . . . . . . . . . . . . . 111

Figure 5.17 Consequences Of The Minimum Ionizing Particle Cut. . . . 112

Figure 6.1 Muon: True Energy vs. Reconstructed Track Length. . . . . 116

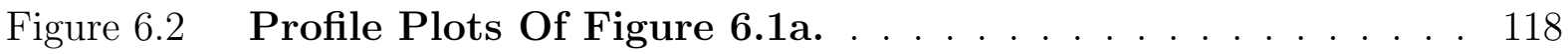

Figure 6.3 Muon Energy Resolution. . . . . . . . . . . . . . . . . . . . 119

Figure 6.4 Muon Energy Distributions. . . . . . . . . . . . . . . . 120

Figure 6.5 Hadronic Energy Classification Zones. . . . . . . . . . . . . . . 122

Figure 6.6 True Hadronic Energy vs. Deposited Energy. . . . . . . . . 123 
Figure 6.7 Hadronic Energy Resolution. . . . . . . . . . . . . . . . . 124

Figure 6.8 Hadronic Energy Distributions. . . . . . . . . . . . . . . 125

Figure 6.9 Quasi-elastic And Non-quasi-elastic Hadronic Energy Distributions. . . . . . . . . . . . . . . . 126

Figure 6.10 Muon And Hadronic Energy Distributions For The Quasielastic And Non-quasi-elastic Events. . . . . . . . . . . 127

Figure 6.11 Muon Energy Resolutions For Quasi-elastic And Non-quasielastic Events. . . . . . . . . . . . . . . . . . . 128

Figure 6.12 Overall Hadronic Energy Resolutions. . . . . . . . . . . . . . 129

Figure 6.13 Neutrino Energy Resolution. . . . . . . . . . . . . . . . 130

Figure 6.14 Neutrino Energy Distributions. . . . . . . . . . . . . . . 131

Figure 6.15 Quasi-elastic And Non-quasi-elastic True Minus Reconstructed Neutrino Energies. . . . . . . . . . . . . . . . . . . . 132

Figure 6.16 Quasi-elastic And Non-quasi-elastic Neutrino Energy Resolutions. . . . . . . . . . . . . . . . . . . . 133

Figure 6.17 Comparison Of Neutrino Energy Resolutions. . . . . . . . . . 134

Figure 6.18 Quasi-elastic And Non-quasi-elastic Neutrino Energy Distributions. . . . . . . . . . . . . . . . . . 134

Figure 6.19 Neutrino Types: Fractions And Energy Distributions. . . . 135

Figure $7.1 \quad$ Mean Energy Deposition Per Unit Length. . . . . . . . . . . 139

Figure 7.2 Comparison Of Data And Monte Carlo Simulated Longest Track Length Distributions. . . . . . . . . . . . . . . 140

Figure 7.3 Energy Estimation Systematic Uncertainty As A Function Of The Neutrino Energy. . . . . . . . . . . . . . . . . . 140

Figure 7.4 Channels Configurations Systematic Uncertainty As A Function Of The Neutrino Energy. . . . . . . . . . . . . . . . 142

Figure $7.5 \quad$ Example Of The $R$ eWeight Package Output. . . . . . . . . 144 
Figure 7.6 GENIE Parameters Systematic Uncertainty As A Function

Of The Neutrino Energy. . . . . . . . . . . . . . . . . . . 146

Figure 7.7 GENIE Parameters Total Systematic Uncertainty As A Function Of The Neutrino Energy. . . . . . . . . . . . . . . . . 146

Figure 7.8 Unfolding Algorithm Systematic Uncertainty As A Function Of The Neutrino Energy. . . . . . . . . . . . . . . . . . 147

Figure 7.9 Summary Of Systematic Uncertainties. . . . . . . . . . . . 149

Figure 7.10 Systematic Uncertainty On The Number Of Events As A Function Of The Neutrino Energy. . . . . . . . . . . . . 150

Figure $7.11 \delta N / N$ As A Function Of The Neutrino Energy. . . . . . . 151

Figure $8.1 \quad$ Angles Of The Longest Track. . . . . . . . . . . . . . . 152

Figure 8.2 Longest Track Angular Distributions Without Cosmic Background. . . . . . . . . . . . . . . . . . . 154

Figure 8.3 Reconstructed Muon Energy Distributions, No Cosmic Background. . . . . . . . . . . . . . . . . 155

Figure 8.4 Cumulative Distribution Functions For Muons. . . . . . . . . 157

Figure 8.5 Hadronic Energy Distributions, No Cosmic Background. . . 157

Figure 8.6 Selected Candidate Events Energy Distributions. . . . . . 158

Figure 8.7 Efficiency And Purity Matrix For The Quasi-elastic And Non-quasi-elastic Samples. . . . . . . . . . . . . . . . . 160

Figure 8.8 Sample Of Simulated Quasi-elastic Neutrino Events. . . . . . 162

Figure 8.9 Sample Of Simulated Non-quasi-elastic Neutrino Events. . . 164

Figure 8.10 Sample Of Quasi-elastic Neutrino Candidates. . . . . . . . 166

Figure 8.11 Sample Of Non-quasi-elastic Neutrino Candidates. . . . . . . 167

Figure 8.12 Number Of Neutrino Candidates vs. Neutrino Energy, No

Cosmic Background. . . . . . . . . . . . . . . . . . . . . . . 169 
Figure 8.13 Number Of Neutrino Candidates vs. Neutrino Energy, No Cosmic Background, Shape Comparison. . . . . . . . . . . 169

Figure 8.14 Quasi-elastic And Non-quasi-elastic Unfolded Number Of Neutrino Candidates vs. Neutrino Energy, No Cosmic Background.

Figure 8.15 Quasi-elastic And Non-quasi-elastic Unfolded Number Of Neutrino Candidates vs. Neutrino Energy, No Cosmic Background, Shape Comparison. . . . . . . . . . . . . . . 171

Figure 8.16 Number Of Neutrino Candidates vs. Neutrino Energy, Comparison Of Monte Carlo Simulation And Data. . . . . . . . . 172

Figure 8.17 Unfolded Number Of Neutrino Candidates vs. Neutrino Energy, Comparison Of Monte Carlo Simulation And Data. . . 172

Figure 9.1 Charged Kaon Longitudinal And Transverse Momenta. . . . 177

Figure 9.2 Ratio Of Neutrinos From Charged Kaon Decays Over Neutrinos From Charged Pion Decays After Event Selection Criteria Applied. . . . . . . . . . . . . . . . . . . 178

Figure 9.3 GENIE Cross Sections As A Function Of Neutrino Energy. 183

Figure 9.4 GENIE Uncertainty In The Muon Neutrino Cross Section. 183

Figure 9.5 Reconstruction Efficiency As A Function Of The Neutrino Energy. . . . . . . . . . . . . . . . . . . . . 184

Figure 9.6 Total Flux Of Muon Neutrinos Coming From Charged Kaon Decays. . . . . . . . . . . . . . . . . . 185

Figure $9.7 \quad$ Flux Systematic Uncertainties. . . . . . . . . . . . . . 186

Figure 9.8 Data Over Monte Carlo Simulated Flux Ratio. . . . . . . . 187

Figure 9.9 Total Flux Of Muon Neutrinos Coming From Charged Kaon Decays Discriminated By Interaction Type. . . . . . . . . . 187

Figure 9.10 Quasi-elastic Over Non-quasi-elastic Flux Ratio. . . . . . . 188

Figure 9.11 Muon Neutrino Monte Carlo Simulated Flux Prediction. . . 191 
Figure 9.12 Reconstruction Efficiency As A Function Of The Neutrino

Energy. . . . . . . . . . . . . . . . . . . . . 191

Figure 9.13 Inclusive Muon Neutrino Charged Current Cross Section

Per Nucleon On A Carbon Target. . . . . . . . . . . . . . . . 192

Figure 9.14 Cross Section Systematic Uncertainties. . . . . . . . . . . . . 193

Figure 9.15 Data Over Monte Carlo Simulated Muon Neutrino Charged

Current Cross Section Ratio. . . . . . . . . . . . . . . . . 193

Figure 9.16 Measurements Of Muon Neutrino Charged Current Inclusive Scattering Cross Sections. . . . . . . . . . . . . . . 194

Figure A.1 Higgs Scalar Potential. . . . . . . . . . . . . . . . . 200

Figure B.1 Sample Event Illustrating The Tracking Algorithm. . . . . . 205

Figure B.2 Flow Chart Of The Tracking Algorithm. . . . . . . . . 206

Figure B.3 Sample Event Of The Tracking Algorithm Output. . . . . . 208

Figure C.1 Neutrino Energy Distributions Before And After Unfolding. 217

Figure C.2 Results From Unfolding Fake Data. . . . . . . . . . . . . 218 


\section{Chapter 1}

\section{Introduction}

Neutrinos are among the most mysterious of all the known particles. Neutrinos are weakly interacting particles, which makes them extremely hard to detect, and yet neutrinos participate in a wide range of phenomena from the subatomic to the cosmological scales. Neutrinos are key to type-II supernovae core collapse since these carry out of the exploding star about $99 \%$ of the gravitational energy release [1]. Neutrinos actively participate in the cooling of stars that passed the He-burning stage and therefore strongly determine the lifetime of the star [2]. Their finite mass presents the first evidence of physics beyond the Standard Model, since the latter assumes that neutrinos are massless. This leads to ideas that would extend the number of fundamental particles providing a mechanism for neutrinos to acquire their mass; however through a mechanism not described in the Standard Model. As massive and extremely abundant particles, neutrinos contribute significantly to the evolution of the Universe [3]. Massive neutrinos exhibit oscillations that change their identities, and the study

of this phenomenon could provide direction in the solutions of current mysteries, e.g. the origin of the matter-antimatter asymmetry of the observable Universe. 


\subsection{Brief History Of Neutrinos}

In the 1930's experimental results showed that electrons in beta decays:

$$
n \rightarrow p+e^{-}+\text {energy, }
$$

were not monochromatic, but rather had a discrete energy spectrum. At that time, the origins of the beta decay were unknown. However, it was certain that the final products of the decay were a proton and an electron. Therefore, their energies ought to be fixed. To explain the undetectable energy, N. Bohr suggested that the concept of conservation of energy could be discarded. W. Pauli addressed his colleagues as "liebe radioaktive Damen un Herren" [4] during the 1930 Tübingen meeting to share with them his deep concern about the continuum energy spectrum of electrons. Pauli suggested that an undetectable particle carried away the missing energy, a hypothesis that would never be verified, should the particle be truly undetectable. E. Fermi named Pauli's hypothetical particle the neutrino ${ }^{1}$. Over twenty years later, F. Reins and C. L. Cowan [5] finally discovered the neutrino. The neutrinos ${ }^{2}$ emitted by a nuclear reactor interacted with protons in two tanks of water, creating neutrons and positrons. The gamma rays created in the annihilation of the positrons were detected in the interspersed tanks of liquid scintillator. These neutrinos were later identified as the electron neutrinos.

Neutrinos are produced in both pion and muon decays. However, it was not clear if the neutrinos in these two decays were all electron neutrinos, or some belonged to a different type of neutrinos. In 1962, L. Lederman and J. Steinberger discovered that there existed two

\footnotetext{
${ }^{1}$ Italian for little neutral one, to distinguish it from the much heavier neutron.

${ }^{2}$ The reaction is: $\bar{\nu}_{e}+p \rightarrow n+e^{+}$, with antineutrinos.
} 
types of neutrinos [6], the electron neutrino $\left(\nu_{e}\right)$ and the muon neutrino $\left(\nu_{\mu}\right)$. The 1988 Nobel Prize in physics was awarded to them for their discovery [7]. At this time, a model of hadrons and leptons included: the electron, the muon, their corresponding neutrinos, and the up, down, and strange quarks. The absence of kaon decays to a down quark via a strangeness changing neutral current, along with symmetry arguments, suggested the existence of a fourth quark [8], charm. This hypothesis was verified by the discovery of the bound state of charm-anticharm quarks, the $\psi / J$ meson $[9,10]$. By 1977 the existence of the third family was confirmed with the discovery of the bottom quark at Fermilab, by L. Lederman's team [11], and the discovery of the tau lepton, by the SLAC-LBL team [12]. Due to the rarity of tau neutrinos $\left(\nu_{\tau}\right)$ in neutrino beams, it took over twenty years to experimentally confirm its existence, by the DONUT collaboration, in 2000 [13].

\subsection{Motivations For The $\mathrm{NO} \nu \mathrm{A}$ Experiment}

Decades of speculation about massive neutrinos ${ }^{3}$ ended when Super-Kamiokande [16] and the Sudbury Neutrino Observatory [17] first reported strong evidence of atmospheric and solar neutrino oscillations, respectively. Neutrino oscillations are only possible if neutrinos are massive. The Super-Kamiokande result presented evidence that $\nu_{\mu}$ oscillate primarily into $\nu_{\tau}$, however they did not achieve a conclusive result on the $\nu_{\mu}$ to $\nu_{e}$ oscillation. The lack of evidence in the latter oscillation channel motivated, at that time, the neutrino community to address the role of the $\nu_{e}$ in oscillations phenomena. Three experiments: Double Chooz [18], Daya Bay [19], and RENO [20], presented positive results in 2012 on the $\nu_{\mu} \rightarrow \nu_{e}$

\footnotetext{
${ }^{3}$ B. Pontecorvo first suggested, in 1957, the concept of neutrino oscillations [14]. R. Davis found the first experimental indication of neutrino oscillations with his Homestake experiment, where a third of the expected flux of neutrinos from the Sun was measured [15]. This is known as the solar neutrino problem.
} 
oscillation channel showing that the oscillation indeed occurs. This last result presents an unrivaled opportunity to study the occurrence of CP violation in the neutrino sector [21].

The NuMI Off-Axis $\nu_{e}$ Appearance $(\mathrm{NO} \nu \mathrm{A})$ experiment, will study the neutrino oscillations phenomenon. $\mathrm{NO} \nu \mathrm{A}$ will investigate the oscillations of $\nu_{\mu}$ to $\nu_{e}$, and muon antineutrinos $\left(\bar{\nu}_{\mu}\right)$ to electron antineutrinos $\left(\bar{\nu}_{e}\right)$, using a neutrino beam produced at Fermilab and detected at the $\mathrm{NO} \nu \mathrm{A}$ far detector located near Ash River, Minnesota. $\mathrm{NO} \nu \mathrm{A}$ will determine any differences that occur in the oscillations of neutrinos and antineutrinos when the beam travels through matter. The neutrino beam at Fermilab is designed to run either in primarily neutrino or primarily antineutrino modes. Studying the differences between the interactions of neutrinos and antineutrinos with matter will help to address the following topics:

- The mass ordering of the three neutrino mass eigenstates.

- The value of a $\mathrm{CP}$ violating phase in the neutrino sector.

- Smaller uncertainties in the current values of the neutrino oscillation parameters.

The quarks and charged leptons sectors exhibit normal mass orderings, i.e. the member of the third family is much more massive than the members of the other two families. In the neutrino sector, however, the possibility of one light and two (quasi-degenerate) heavier neutrinos could occur ${ }^{4}$. Even though the absolute value of the mass gap between the lightest and heaviest neutrinos is known, it is yet to be determined which eigenstate is the lightest and which is the heaviest. This results in the uncertainty on the sign of the mass difference. This sign has a profound influence in the $\nu_{\mu} \rightarrow \nu_{e}$ oscillation enhancing (+) or suppressing (-) it. $\mathrm{NO} \nu \mathrm{A}$ is going to make a precise measurement of this oscillation channel, which will determine the sign of the mass difference, and thus the neutrino mass ordering.

\footnotetext{
${ }^{4}$ This is know as inverted mass ordering or hierarchy. See figure 2.9.
} 
By taking neutrino and antineutrino measurements, $\mathrm{NO} \nu \mathrm{A}$ will be able to establish whether there is any significant difference in the two oscillation patterns beyond that introduced by propagation of neutrinos through matter, indicating that CP symmetry is violated in neutrino oscillations. Therefore, neutrinos become prime candidates to explain the origin of the observed matter-antimatter asymmetry at the time of the Big Bang.

$\mathrm{NO} \nu \mathrm{A}$ is a two-detector experiment with one located underground at Fermilab (near detector), and the other, with a mass of $14 \mathrm{kton}$, on the surface in northern Minnesota (far detector). The two detectors are constructed from the same materials differing only in mass (300 ton for the near detector), and use the same readout electronics differing only in sampling rate (higher for the near detector). The similarity between the detectors allows the initial event rate of $\nu_{e}$ and $\nu_{\mu}$, measured by the near detector, to yield a nearly biasfree normalization of the event rate at the far detector. The two detectors are $14 \mathrm{mrad}$ off the neutrino beam axis, which provides a narrow neutrino energy spectrum ${ }^{5}$ peaked around $2 \mathrm{GeV}$. At the far detector, a $2 \mathrm{GeV}$ neutrino beam combined with its baseline length of $810 \mathrm{~km}$ produces a maximum in the $\nu_{e}$ appearance probability, and a minimum in the $\nu_{\mu}$ survival probability, with the flux dominated by oscillations to $\nu_{\tau}$. The exclusively neutral current interactions of $\nu_{\tau}$ at this energy, where the scattered $\nu_{\tau}$ carries away a large fraction of the energy, leaves little hadronic energy to produce backgrounds for the $2 \mathrm{GeV}$ $\nu_{e}$ interactions.

The two $\mathrm{NO} \nu \mathrm{A}$ detectors are matrices of PVC tubes filled with liquid scintillator, resulting in detectors with approximately $70 \%$ of their weight being active detecting material. The ionization left by charged particles created in the neutrino interactions generates light in the scintillator, which is collected by a special kind of fiber optics that transport the

\footnotetext{
${ }^{5}$ See figure 3.3.
} 
wavelength-shifted light to avalanche photodiodes. The photodiode electrical pulses are amplified, digitized, and processed by a data acquisition system. The processed data are stored on disc for later analysis.

The NO $\nu$ A collaboration built a prototype detector at Fermilab exposed to the neutrino beam to develop calibration, simulation, and reconstruction tools, using real data. A number of construction issues were identified and solved. These influenced the final design of the near and far detectors. Regardless of the location of the prototype on the surface, the neutrino interaction signals can be separated from the enormous amounts of cosmic rays that continuously illuminate the prototype detector. Cosmic rays are a background to the $\nu_{\mu}$ charged current neutrino signal ${ }^{6}$. The neutrino beam is pulsed at regular intervals, and the analysis of the data finds a peak at the time of the interaction candidates from the neutrino beam. With the ability to distinguish neutrino events from cosmic rays, the prototype is used to make a proof of principle: the collaboration searched for $\nu_{\mu}$ interactions and these were indeed found [22].

The $\nu_{\mu}$ charged current quasi-elastic cross $\operatorname{section}^{7}$ was measured [22] in the $\mathrm{NO} \nu \mathrm{A}$ prototype detector using a selection of events with one and only one reconstructed track. The neutrino energy was determined solely from the range and angle of the reconstructed muon track. Using the calorimetric capabilities of the prototype detector's design, and the neutrino flux embedded in the $\mathrm{NO} \nu \mathrm{A}$ Monte Carlo simulation, this thesis presents an estimate of the inclusive $\nu_{\mu}$ charged current cross section.

Charged kaons produced at the NuMI $\operatorname{target}^{8}$ are the source of the $\nu_{e}$ that contribute

\footnotetext{
${ }^{6}$ See section 2.1.1 for a definition of the charged current interaction.

${ }^{7}$ See section 2.1.1.1 for a definition of the charged current quasi-elastic interaction.

${ }^{8}$ See section 3.1 for a detailed description of the NuMI beam, including the charged kaon production.
} 
to the total background of the $\nu_{\mu}$ beam that reaches the far detector. Knowing the initial content of $\nu_{e}$ in the neutrino beam will minimize the systematic uncertainty in the number of $\nu_{e}$ expected from $\nu_{\mu}$ oscillations. The charged kaon decay peak in the neutrino energy is observed in the inclusive charge current interaction data to be presented. The off-axis angle, and the two-body decay of the mesons, lead to a narrow peak in the energy of the neutrinos at $2 \mathrm{GeV}$. However, the production rate of charged kaons in the NuMI target has to be determined from Monte Carlo simulations as there are no direct measurements. At the location of the prototype detector, the flux of $2 \mathrm{GeV}$ neutrinos is nearly all from the decay of charged kaons. The last two statements lead to a rather large normalization uncertainty in the measured neutrino cross section. From the few other measurements of neutrino cross sections in this energy regime, the level of uncertainty in the flux can be estimated. Therefore, both interpretations of the data, as an inclusive cross section estimate, or as a flux estimate, are presented.

\subsection{Outline}

In order to set the theoretical framework behind the neutrino interactions, chapter 2 presents the basics of the weak interactions, emphasizing those involving neutrinos (section 2.1). In addition, the possible origins of neutrino masses (section 2.2), and a review of the formalism of neutrino oscillations (section 2.3) are presented.

Chapter 3 provides an overview of the Fermilab neutrino beam that supplies the neutrinos to the $\mathrm{NO} \nu \mathrm{A}$ experiment. Sections 3.1 and 3.2 summarize the components of the neutrino beam, and the various steps involved in the neutrino production. The beam composition and energy spectrum are presented in section 3.3. 
The concepts behind the NO $\nu$ A experiment are presented in chapter 4. Section 4.1 is an overview of the experiment. The prototype detector is introduced in section 4.2. The various software tools used for data processing and analysis are presented in section 4.3. The data quality checks are reviewed in section 4.4. A brief study of cosmic rays, and its implications in the experiment, is provided in section 4.5.

The $\nu_{\mu}$ event selection procedure is described in chapter 5 . The differences between events from charged and neutral current interactions are discussed in section 5.1, the event containment criteria are presented in section 5.2, and the charged current event selection is explained in section 5.3.

The neutrino energy reconstruction process is discussed in chapter 6 . There are two steps in this process: muon energy reconstruction (section 6.1), and the hadronic energy reconstruction (section 6.2). A classification between quasi-elastic and non-quasi-elastic events (section 6.3) is necessary to better reconstruct the neutrino energy (section 6.4).

The various systematic uncertainties associated with the results are detailed in chapter 7. The analysis of the data is presented in chapter 8. A comparison between simulation and data is provided in section 8.1, and the results are presented in section 8.2. Chapter 9 discusses the implications of the results on: the neutrino flux (section 9.1), and the inclusive $\nu_{\mu}$ charged current cross section (section 9.2). Final remarks are presented in section 9.3. 


\section{Chapter 2}

\section{Theoretical Framework for Neutrinos}

The Standard Model provides the theoretical foundations for quantum chromodynamics (QCD), and it also unifies electromagnetism and the weak interaction, based on the works done by S. L. Glashow [23], S. Weinberg [24], and A. Salam [25]. Neutrinos have an important role in the weak interaction, which is briefly reviewed in this chapter. Massive neutrinos are not part of the Standard Model of particle physics. There are a few ideas to explain the origins of neutrino masses, the most popular within the neutrino community is the See Saw Mechanism, also reviewed in this chapter. Neutrino oscillations are one of the most relevant consequences of massive neutrinos, and therefore are reviewed at the end of the chapter.

\subsection{Neutrinos In Weak Interactions}

The electroweak interaction was first developed in a phenomenological fashion, and later complemented when additional measurements became available. In 1934 Fermi [26] introduced an effective Hamiltonian ${ }^{1}$ :

$$
\mathcal{H}_{\mathrm{eff}}=-\frac{G_{F}}{\sqrt{2}} J_{\mu}(x) J_{\mu}^{\dagger}(x),
$$

\footnotetext{
${ }^{1}$ Fermi coupling constant: $G_{F} /(\hbar c)^{3}=1.166 \times 10^{-5} \mathrm{GeV}^{-2}$.
} 
to model the low energy charged current weak interaction of hadrons and leptons known at the time as:

$$
J_{\mu}(x)=\bar{\Psi}_{\nu_{e}} \gamma_{\mu}\left(1-\gamma_{5}\right) \Psi_{e}+\bar{\Psi}_{p} \gamma_{\mu}\left(1-\gamma_{5}\right) \Psi_{n} .
$$

The model resembled that of electromagnetism given its vector part, however it also included an axial part introduced after the discovery of parity violation [27].

At low neutrino energies, charged current interactions are characterized by quasi-elastic scattering with a nucleon and lepton in the final state. Nucleon resonance production becomes important as their thresholds are crossed. At high energies, the cross section grows linearly with energy and becomes dominated by deep inelastic scattering. The formalism describing these interactions is described in the next section.

\subsubsection{Charged Current Interactions}

The interaction of the $W$ boson with fermions has the following Lagrangian:

$$
\mathcal{L}_{W}=-\frac{g}{2 \sqrt{2}}\left(J_{W}^{\mu} W_{\mu}^{-}+J_{W}^{\mu \dagger} W_{\mu}^{+}\right),
$$

where the charged current $(\mathrm{CC})$ is:

$$
\begin{aligned}
J_{W}^{\mu \dagger} & =\sum_{k=1}^{3}\left(\bar{\nu}^{k} \gamma^{\mu}\left(1-\gamma^{5}\right) l^{k}+\bar{u}^{k} \gamma^{\mu}\left(1-\gamma^{5}\right) d^{k}\right) \\
& =\left(\bar{\nu}_{e} \bar{\nu}_{\mu} \bar{\nu}_{\tau}\right) \gamma^{\mu}\left(1-\gamma^{5}\right) U_{l}\left(\begin{array}{l}
e \\
\mu \\
\tau
\end{array}\right)+(\bar{u} \bar{c} \bar{t}) \gamma^{\mu}\left(1-\gamma^{5}\right) V_{q}\left(\begin{array}{l}
d \\
s \\
b
\end{array}\right) .
\end{aligned}
$$


$V_{q}$ is the Cabibbo-Kobayashi-Maskawa (CKM) matrix $[28,29]$, and $U_{l}$ is the PontecorvoMaki-Nakagawa-Sakata (PMNS) leptonic mixing matrix [30,31]. The vertices of interest, from equations $(2.3,2.4)$, are: $\bar{d}_{j} u_{i} W^{-}, \bar{u}_{i} d_{j} W^{+}, \bar{l}_{j} \nu_{i} W^{-}$, and $\bar{\nu}_{i} l_{j} W^{+}$, which contribute to the neutrino scattering with nucleons. The first two vertices correspond to the lower vertex in figure 2.1, and the last two correspond to the upper vertex in figure 2.1. The interaction: $\nu_{\mu}+d \rightarrow \mu^{-}+u$, which is the underlying process of the $\nu_{\mu}+n \rightarrow \mu^{-}+p$ scattering, is an example of the interactions that are generated with the four vertices mentioned above.

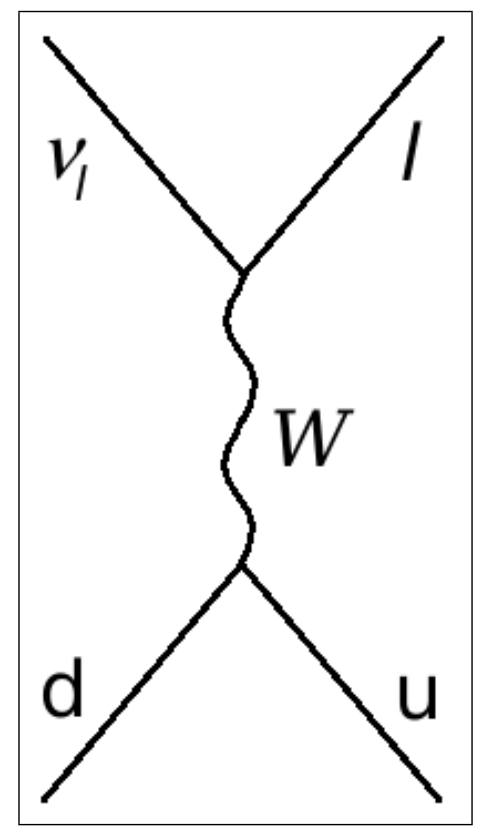

Figure 2.1 Weak Interaction Charged Current Representation Of Neutrinonucleon Scattering. In the drawing the index $l$ represents all charged leptons, the letter $d$ represents all the $Q=-e / 3$ quarks, and the letter $u$ represents all the $Q=2 e / 3$ quarks.

\subsubsection{Quasi-elastic Scattering}

For energies smaller than the $W$ mass, the amplitude $(\mathcal{M})$ :

$$
\mathcal{M}=\frac{G_{F}}{\sqrt{2}} \bar{l} \gamma_{\mu}\left(1-\gamma_{5}\right) \nu\left\langle p\left|J_{\mu}^{+}\right| n\right\rangle,
$$


of the neutrino interactions ${ }^{2}$ :

$$
\begin{aligned}
& \nu_{l}(\mathbf{k})+n(\mathbf{p}) \rightarrow l^{-}\left(\mathbf{k}^{\prime}\right)+p\left(\mathbf{p}^{\prime}\right) \\
& \bar{\nu}_{l}(\mathbf{k})+p(\mathbf{p}) \rightarrow l^{+}\left(\mathbf{k}^{\prime}\right)+n\left(\mathbf{p}^{\prime}\right)
\end{aligned}
$$

is the product of the hadronic and leptonic currents [27]:

The general hadronic current gets contributions from the motion of the quarks within the hadrons, hence the introduction of form factors is useful to describe the physics of the $\operatorname{processes}^{3}$ :

$$
\left\langle p\left|J_{\mu}^{+}\right| n\right\rangle=\bar{n}\left(g_{V} \gamma_{\mu}+f_{V} \frac{\mathbf{p}_{\mu}}{2 M_{N}}+h_{V} \frac{\mathbf{q}_{\mu}}{2 M_{N}}+g_{A} \gamma_{\mu} \gamma_{5}+\imath f_{A} \sigma_{\mu \nu} \frac{\mathbf{q}^{\nu} \gamma_{5}}{2 M_{N}}+h_{A} \frac{\mathbf{q}_{\mu} \gamma_{5}}{2 M_{N}}\right) p
$$

Here the $g_{A}, f_{A}$, and $h_{A}$ are axial form factors, and the $g_{V}, f_{V}$, and $h_{V}$ are vector form factors. The product $h_{A} \mathbf{q}_{\mu} \gamma_{5}$ is proportional to the lepton's mass, therefore is a negligible term $^{4}$, and $h_{V}$ and $f_{A}$ need to be zero due to charge conjugation and time-reversal arguments [27]. The simplified form of equation (2.7) is:

$$
\left\langle p\left|J_{\mu}^{+}\right| n\right\rangle=\bar{n}\left(g_{V} \gamma_{\mu}+f_{V} \frac{\mathbf{p}_{\mu}}{2 M_{N}}+g_{A} \gamma_{\mu} \gamma_{5}\right) p
$$

The kinematics for the processes (see equation (2.6)) in the laboratory frame, for which the

\footnotetext{
${ }^{2} \mathbf{k}$ and $\mathbf{p}$ are momenta.

${ }^{3} M_{N}$ is the mass of the target nucleon.

${ }^{4}$ True for light leptons.
} 
nucleon is at rest, are given by:

$$
\begin{aligned}
\mathbf{p} \cdot \mathbf{k} & =\mathbf{p}^{\prime} \cdot \mathbf{k}^{\prime}=M_{N} E_{\nu}, \\
\mathbf{p} \cdot \mathbf{k}^{\prime} & =\mathbf{p}^{\prime} \cdot \mathbf{k}=M_{N} E_{\nu}+\frac{\mathbf{q}^{2}}{2}, \\
\mathbf{k} \cdot \mathbf{k}^{\prime} & =\frac{m_{l}^{2}}{2}-\frac{\mathbf{q}^{2}}{2}, \\
\mathbf{p} \cdot \mathbf{p}^{\prime} & =\frac{M_{W}^{2}}{2}-\frac{\mathbf{q}^{2}}{2}, \\
Q^{2} & =4 E_{\nu} E^{\prime} \sin ^{2}\left(\frac{\theta}{2}\right),
\end{aligned}
$$

where $E_{\nu}$ is the energy of the incoming $\nu_{l}, E^{\prime}$ is the energy of the outgoing $l, Q^{2}$ is the momentum transfer, and $\theta$ is the angle between the momenta of the incoming neutrino and the outgoing charged lepton. Equations $(2.8,2.9)$ lead to the differential cross section:

$$
\begin{aligned}
\frac{d \sigma_{\mathrm{QE}}}{d E^{\prime}}= & \frac{G_{F}^{2} M_{N}}{4 \pi}\left[\left(g_{V}-g_{A}\right)^{2}+\left(g_{V}+g_{A}\right)^{2}\left(\frac{E^{\prime}}{E_{\nu}}\right)^{2}+\left(g_{V}^{2}-g_{A}^{2}\right) \frac{M_{N}}{E_{\nu}^{2}}\right] \\
+ & \frac{G_{F}^{2} M_{N}}{8 \pi}\left[f_{V}^{2} \eta+2 f_{V} g_{V}\right]\left[\left(1+\frac{E^{\prime}}{E_{\nu}}\right)^{2}-\frac{Q^{2}}{E_{\nu}^{2}} \eta\right],
\end{aligned}
$$

with:

$$
\eta=1+\frac{Q^{2}}{4 M_{N}^{2}} .
$$

Monte Carlo simulated quasi-elastic cross sections based on this function provide a reasonable representation of the data from experiments (MiniBooNE [32], ANL [33], BEBC [34], BNL [35, 36], FNAL [37], Gargamelle [38, 39], LSND [40], NOMAD [41], Serpukhov [42], and SKAT [43]), as seen in figure 2.2. The two solid curves are the Monte Carlo simulation predictions of the cross sections based on equation (2.10). The quasi-elastic cross sections become constant for energies above $10 \mathrm{GeV}$. 


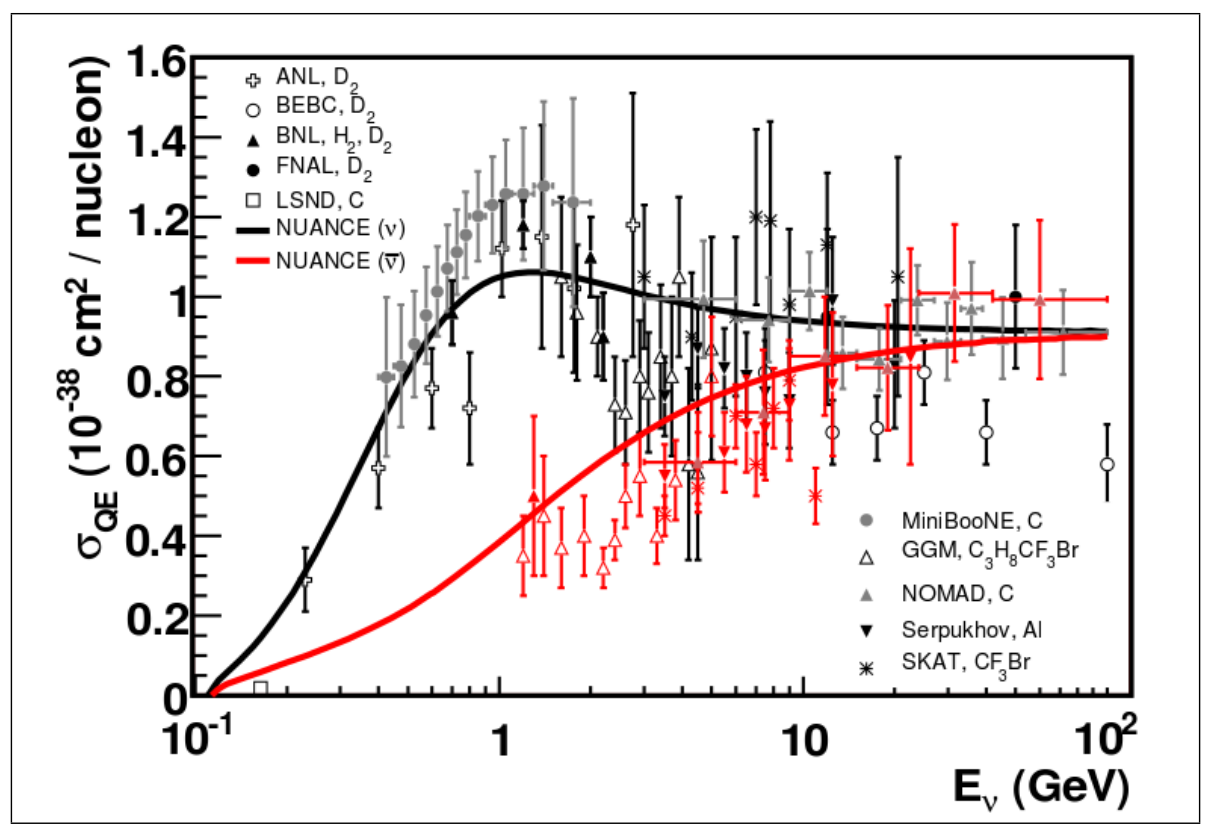

Figure 2.2 Muon Neutrino Quasi-elastic Cross Sections. Measurements of the $\nu_{\mu}$ (black) and $\bar{\nu}_{\mu}$ (red) quasi-elastic scattering cross sections (per nucleon) as a function of neutrino energy [44].

\subsubsection{Resonant Pion Production}

As neutrino energy gets larger, their scattering with nucleons can excite the latter to higher energy levels; these interactions produce baryon resonances $\left(N^{*}\right)$ :

$$
\nu_{\mu} N \rightarrow \mu^{-}+N^{*},
$$

which rapidly decays (often) into a nucleon and a charged pion:

$$
N^{*} \rightarrow \pi+N^{\prime},
$$

where $N$ and $N^{\prime}$ could be either a neutron or a proton in a nucleus. Higher order multiplicity decay modes are also possible. These resonances were studied by D. Rein and L. M. Sehgal 

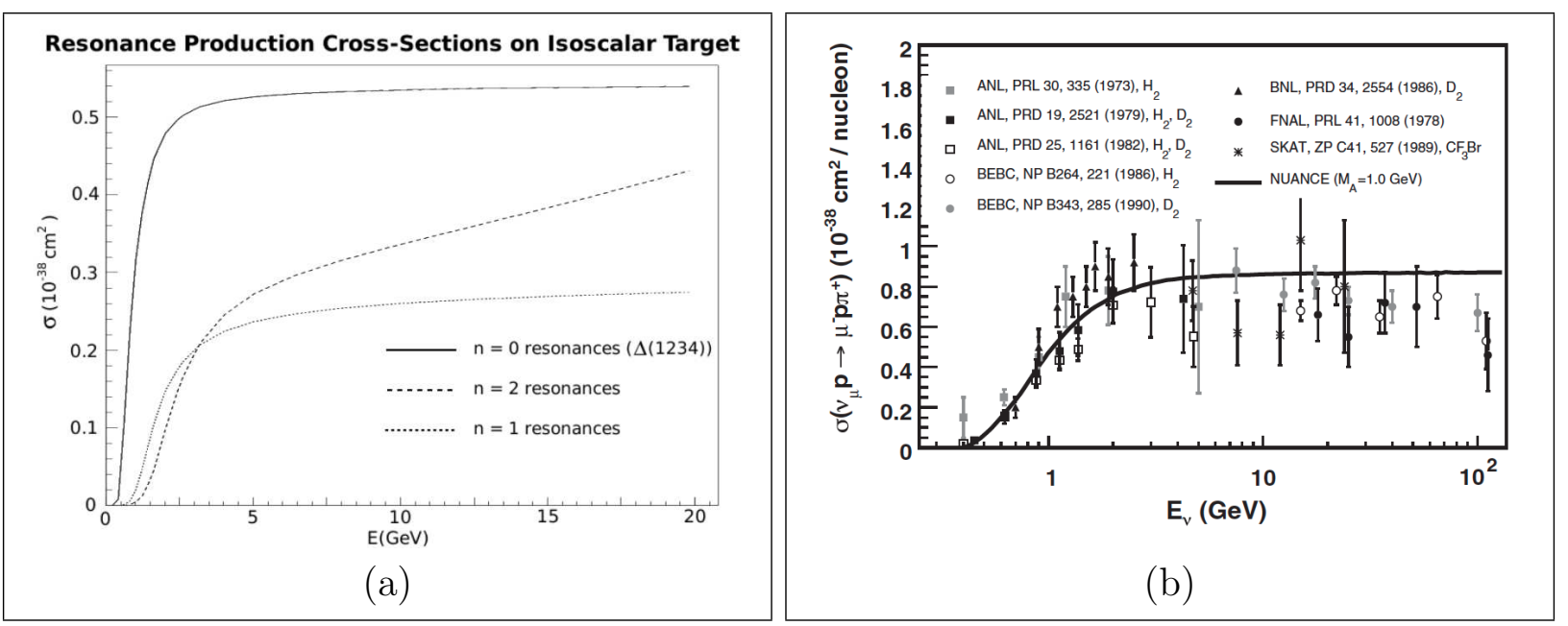

Figure 2.3 Muon Neutrino Resonance Cross Sections. (a) Resonance production cross section for differing $n_{H}$ [47]. (b) Existing measurements of the cross section for the process in equation (2.15) as a function of energy [48].

[45], based on the model of baryon resonances of R. Feynman, M. Kislinger, and F. Ravndal [46]. Their work describes the resonances in terms of excited states of three bounded quarks $(a, b$, and $c)$ with a relativistic harmonic oscillator potential:

$$
H_{\mathrm{res}}=3\left(\mathbf{p}_{a}^{2}+\mathbf{p}_{b}^{2}+\mathbf{p}_{c}^{2}\right)+\frac{1}{36} \Omega^{2}\left[\left(\mathbf{u}_{a}-\mathbf{u}_{b}\right)^{2}+\left(\mathbf{u}_{b}-\mathbf{u}_{c}\right)^{2}+\left(\mathbf{u}_{c}-\mathbf{u}_{a}\right)^{2}\right],
$$

with $\Omega^{2}=m^{2} \omega_{0}^{2}$, the $\mathbf{u}^{\prime}$ s are conjugate positions of the quarks, and the p's are fourmomentum vectors of the quarks ${ }^{5}$. The eigenvalues of the harmonic oscillator presented in [46] are a succession of integers, $n_{H}$, times $\Omega$. The first state, $n_{H}=0$, is the resonance $\Delta(1232)$, and its cross section saturates at energies around $2 \mathrm{GeV}$. In the cases where $1 \leq n_{H} \leq 2$, the theoretical cross sections for: $n_{H}=1$ saturates at energies higher than $20 \mathrm{GeV}$, and $n_{H}=2$ rises linearly with energy, as seen in figure $2.3 \mathrm{a}$. Measurements of the cross section for the process:

$$
\nu_{\mu}+p \rightarrow \mu^{-}+p+\pi^{+}
$$

\footnotetext{
${ }^{5} \mathbf{u}$ 's and p's defined in [46].
} 
have been made in bubble chamber experiments, and the results are shown in figure 2.3b.

\subsubsection{Deep Inelastic Scattering}

When hadron structures are examined on a very short distance scale, it is found that these are a set of non-interacting quarks. Short distance, in this case, translates to high energy electroweak interactions in deep inelastic (lepton-hadron) scattering of the form:

$$
l+N \rightarrow l^{\prime}+\mathrm{X}
$$

where X includes single nucleon or nucleon resonance production. The kinematics of these processes are described in terms of the following variables (recall equations (2.9)):

$$
\tilde{x}=\frac{Q^{2}}{2 M_{N} E y}, \quad y=\frac{E_{\mathrm{had}}}{E_{\nu}},
$$

where $E_{\text {had }}$ is the energy of the hadronic system. Using these variables, the deep inelastic scattering (DIS) double differential cross section is:

$$
\frac{d^{2} \sigma}{d \tilde{x} d y}=\frac{G_{F}^{2} M_{N} E_{\nu}}{\pi\left(1+\frac{Q^{2}}{M_{W}^{2}}\right)^{2}}\left[2 \tilde{x} \frac{y^{2}}{2}\left(g_{V}+f_{V}\right)-f_{V}\left(1-y-\frac{M_{N} \tilde{x} y}{2 E_{\nu}}\right)\right]
$$

Equation (2.18) is the core of the physics involved in DIS. However, additional effects must be included in any realistic description. The inclusion of non-perturbative higher twist effects [49], heavy quark production [50], target mass effects [51], nuclear effects and radiative corrections [52], higher order QCD processes [53], and lepton masses [54], further modify the scattering cross section in equation (2.18). In general, these contributions are known with 
reasonable uncertainties [48].

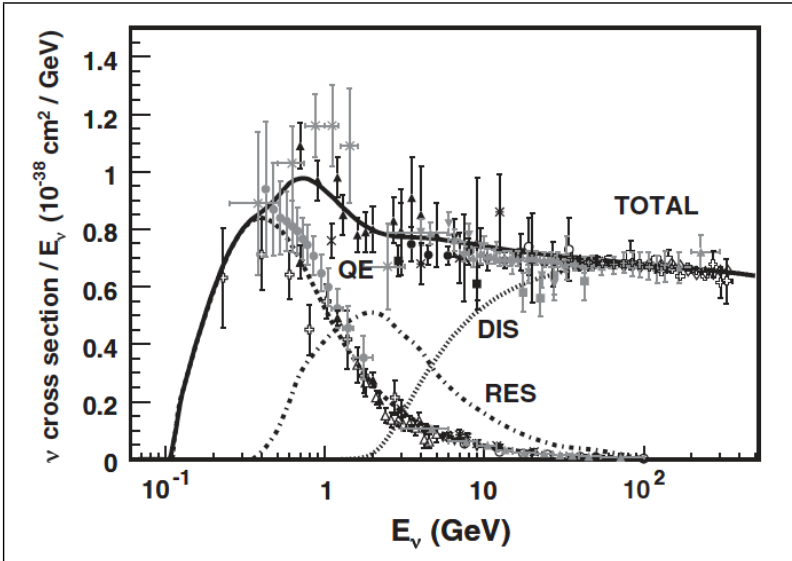

(a)

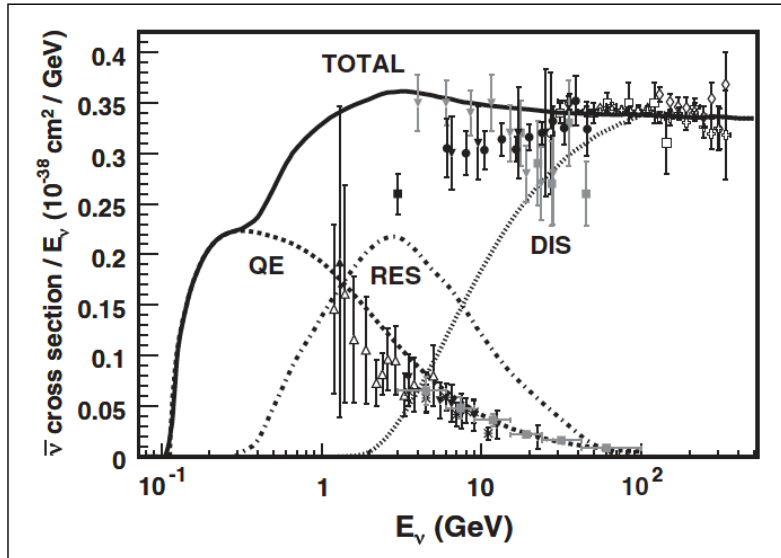

(b)

Figure 2.4 Total Inclusive Muon Neutrino Charged Current Cross Sections. Total (inclusive) (a) $\nu_{\mu}$ and (b) $\bar{\nu}_{\mu}$ charged current cross sections per nucleon over neutrino energy as a function of neutrino energy. The total cross sections (solid lines) are the sum of: QE (dashed), resonance (dot-dashed), and deep inelastic (dotted) [48].

A collection of data acquired over the last 50 years, using different experimental techniques [48], is presented in figure 2.4. These figures show the measured and predicted QE and inclusive $\mathrm{CC}$ cross sections. For energies higher than $3 \mathrm{GeV}$ the inclusive cross section is dominated by the DIS processes, as can be seen in the region where the ratio $\sigma_{D I S} / \sigma_{\mathrm{QE}}>1$. To isolate the DIS events, neutrino experiments usually apply kinematic cuts to remove QE scattering and resonance contributions from the data.

\subsubsection{Inclusive Cross Section Measurements}

There are only two experiments that have measured the $\nu_{\mu} \mathrm{CC}$ inclusive cross section at neutrino energies around $2 \mathrm{GeV}$ : Brookhaven's $7 \mathrm{ft}$ bubble chamber [55] (1982) and SciBoone (2011). The experiment at Brookhaven used a $7 \mathrm{ft}$ deuterium bubble chamber exposed to the Alternating Gradient Synchrotron wide-band neutrino beam. The value of the inclusive cross section divided by the neutrino energy falls significantly from $0.4 \mathrm{GeV}$ to $1.5 \mathrm{GeV}$, and then 
becomes constant, as seen in figure 2.5. Above $1.6 \mathrm{GeV}$, the measured inclusive $\mathrm{CC}$ cross section per nucleon divided by the neutrino energy is $\sigma / E=(0.80 \pm 0.03) \times 10^{-38} \times \mathrm{cm}^{2} / \mathrm{GeV}$ [55], the average of the values shown in figure 2.5 .

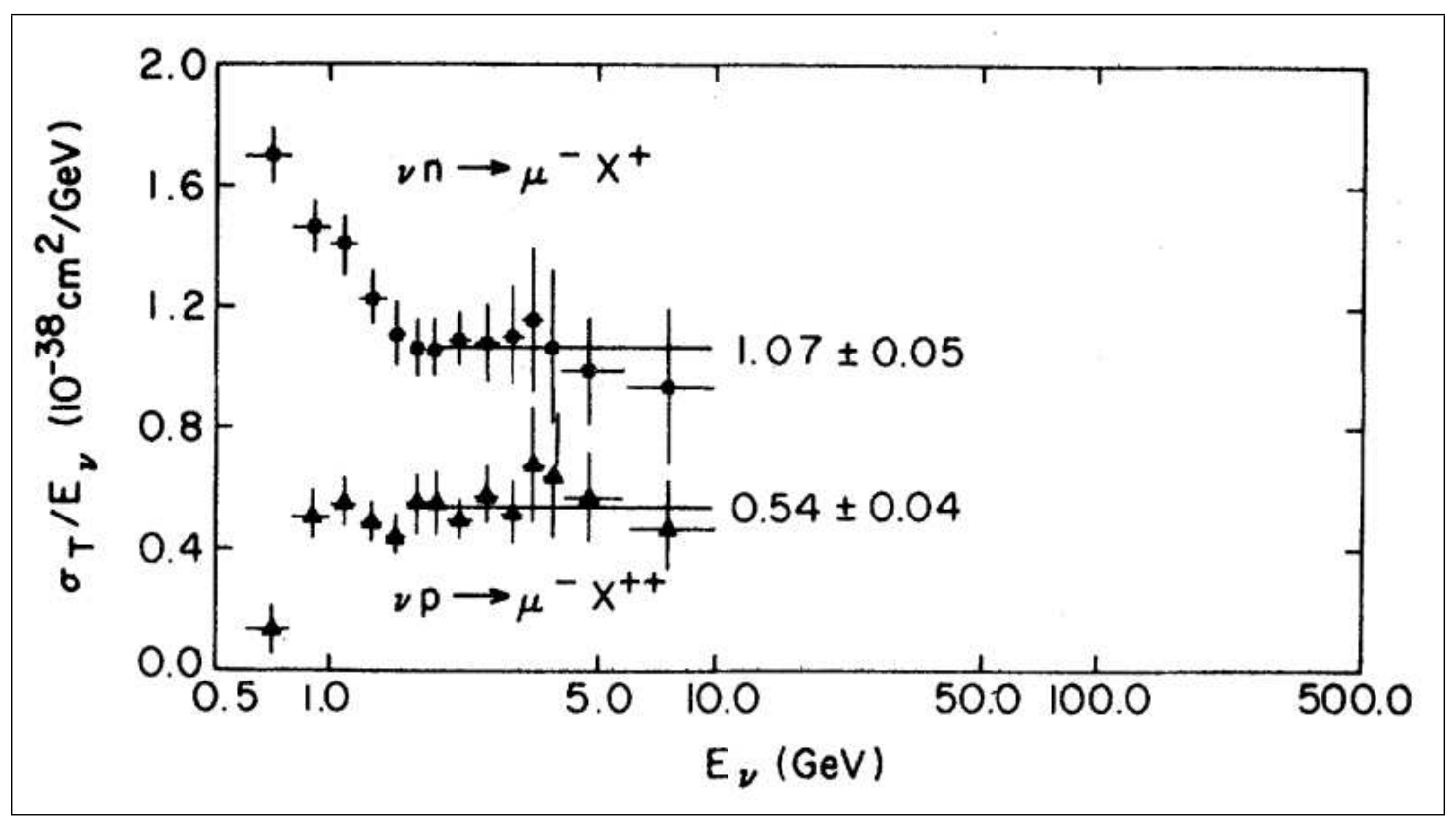

Figure 2.5 Brookhaven National Laboratory Muon Neutrino Inclusive Cross Sections. $\nu_{\mu}$ inclusive cross section over neutrino energy as a function of the neutrino energy for: $\nu p$ and $\nu n$ interactions. The solid lines are the best fits for neutrino energies higher than $1.6 \mathrm{GeV}[55]$.

At neutrino energies around $2 \mathrm{GeV}$, nuclear effects are important. When the $W$ boson's four-momentum is low enough such that its de Broglie wavelength is comparable to the size of the target nucleus, the scattering involves the entire nucleus. The cross sections on deuterium targets are not directly applicable to the heavier nuclear target materials used in current accelerator-based neutrino experiments. In neutrino scattering on a nucleon (in a nuclear target), if there is a small momentum transfer to the final state nucleon, this can only exist in excited states allowed by Pauli's Exclusion Principle. In the Fermi gas model, the excited nucleons undergo transitions across the Fermi surface, with momentum around 


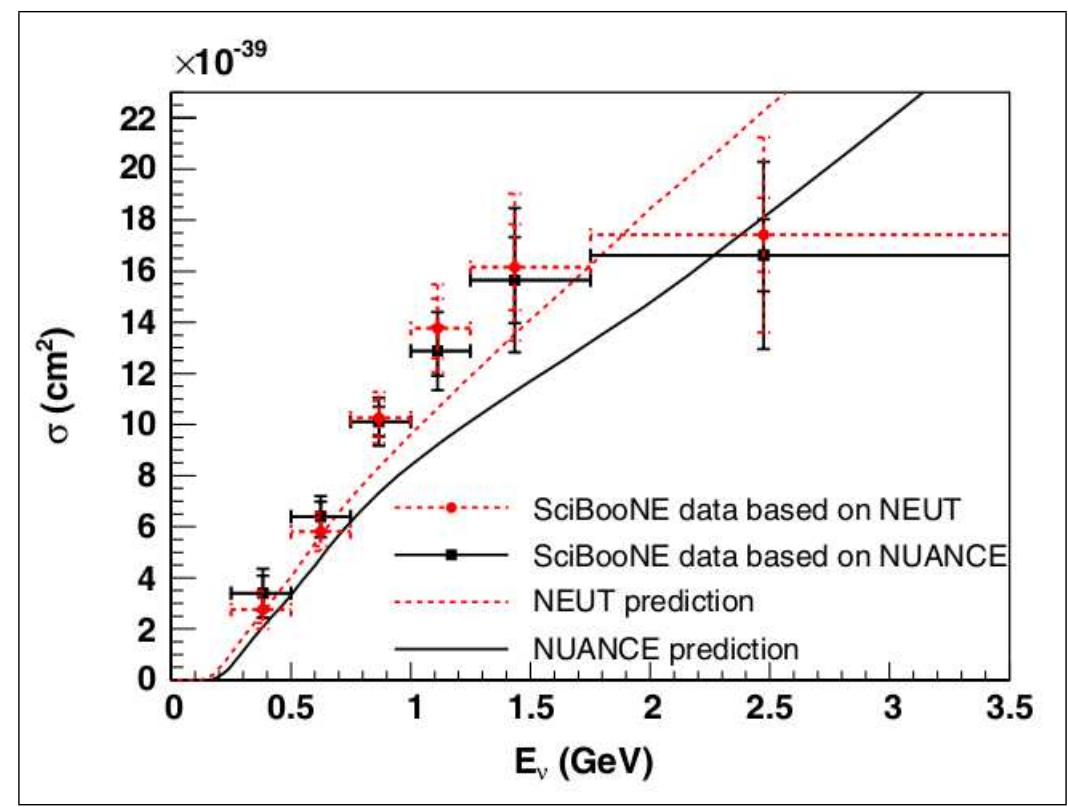

Figure 2.6 SciBooNE Muon Neutrino Inclusive Cross Sections. $\nu_{\mu}$ inclusive cross section per nucleon on a polystyrene target $\left(\mathrm{C}_{8} \mathrm{H}_{8}\right)$ [56]. The NEUT (dashed red line) [57] and NUANCE (black line) [58] predictions are shown for reference.

$250 \mathrm{MeV} / \mathrm{c}$, from low energy states, which are all occupied, to high energy states. Thus, the neutrino-nucleon interaction can only occur if there is an available higher energy state for the nucleon to occupy. This is known as Pauli-Blocking [59]. When the nucleon involved in the scattering interacts with other nucleons, the kinematics and multiplicity of the final state are diverse. SciBoone measured the inclusive $\nu_{\mu} \mathrm{CC}$ interactions, using the Fermilab Booster neutrino beam, on a polystyrene target. Their results are shown in figure 2.6. The rise of the cross section with energy agrees somewhat better with the predictions of the NEUT simulation. The smaller error bars show the uncertainties of the rate normalization factors and the larger error bars represent the total error including the flux uncertainties.

The two results presented in this section are consistent with each other, and are useful to tune various neutrino interaction models in the $2 \mathrm{GeV}$ region. 


\subsubsection{Neutral Current Interactions}

Though hints for the existence of neutrino scattering without final state leptons had been around for a number of years, compelling experimental evidence for weak neutral current interactions [60] became available at about the same time as the electroweak unification theory was proposed, and it depended crucially on the existence of weak neutral currents.

The fermionic Lagrangian ${ }^{6}$ describes neutral and charged currents. There is a Lagrangian, equivalent to that in equation (2.3), that describes the interaction of the $Z$ boson with fermions, given by [27]:

$$
\begin{aligned}
\mathcal{L}_{Z}= & \frac{G_{F}}{\sqrt{2}} \sum_{k=1}^{3} \bar{\nu}^{k} \gamma^{\mu}\left(1-\gamma^{5}\right) \nu^{k}\left\{\bar{u}^{k} \gamma^{\mu}\left[u_{L}^{k}\left(1-\gamma^{5}\right)+u_{R}^{k}\left(1+\gamma^{5}\right)\right] u^{k}\right\} \\
+ & \frac{G_{F}}{\sqrt{2}} \sum_{k=1}^{3} \bar{\nu}^{k} \gamma^{\mu}\left(1-\gamma^{5}\right) \nu^{k}\left\{\bar{d}^{k} \gamma^{\mu}\left[d_{L}^{k}\left(1-\gamma^{5}\right)+d_{R}^{k}\left(1+\gamma^{5}\right)\right] d^{k}\right\} .
\end{aligned}
$$

The vertices of interest that come out from equation (2.19) are: $\bar{u}_{j} u_{i} Z, \bar{d}_{j} d_{i} Z$, and $\bar{\nu}_{j} \nu_{i} Z$, which contribute to the neutrino scattering with nucleons. The first two vertices correspond to the lower vertex in figure 2.7, and the last vertex corresponds to the upper vertex in figure 2.7. The interaction $\nu_{\mu}+d \rightarrow \nu_{\mu}+d$ is an example of the scattering that is generated with the three vertices mentioned above.

Equations $(2.9,2.19)$ lead to the elastic neutral current differential cross section [48]:

$$
\frac{d \sigma}{d Q^{2}}=\frac{G_{F}^{2} M_{N}^{2}}{8 \pi E_{\nu}^{2}}\left[A \pm \frac{4 M_{N} E_{\nu}-Q^{2}}{M_{N}^{2}} B+\frac{\left(4 M_{N} E_{\nu}-Q^{2}\right)^{2}}{M_{N}^{4}} C\right]
$$

\footnotetext{
${ }^{6}$ See equation (A.12).
} 


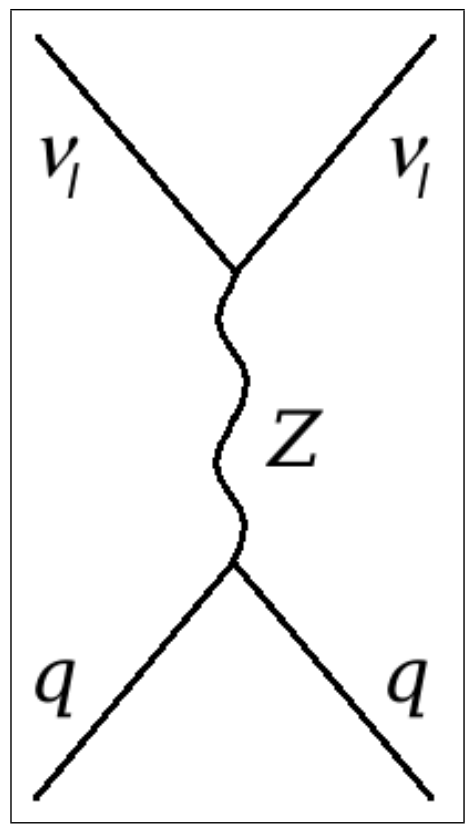

Figure 2.7 Weak Interaction Neutral Current Representation Of Neutrino-nucleon Scattering. In the drawing the index $l$ represents all charged leptons, the letter $q$ represents all quarks.

with:

$$
\begin{aligned}
A & =\frac{Q^{2}}{M_{N}^{2}}\left[g_{A}^{2} \eta_{+}-\left(g_{V}+f_{V}\right)^{2} \eta_{-}+f_{V}^{2} \frac{Q^{2}}{4 M_{N}^{2}} \eta_{-}+\left(g_{V}-f_{V}\right) f_{V} \frac{Q^{2}}{M_{N}^{2}}\right] \\
B & =\frac{Q^{2}}{M_{N}^{2}} g_{A} f_{V} \\
C & =\frac{1}{4}\left[g_{A}^{2}+\left(g_{V}+f_{V}\right)^{2}+f_{V}^{2}\left(\eta_{-}-1\right)\right] \\
\eta_{ \pm} & =1 \pm \frac{Q^{2}}{4 M_{N}^{2}}
\end{aligned}
$$

The MiniBooNE collaboration has published a measurement of the elastic neutral current differential cross section for the process:

$$
\nu+N \rightarrow \nu+N
$$


on a $\mathrm{CH}_{2}$ target [61], as seen in figure 2.8.

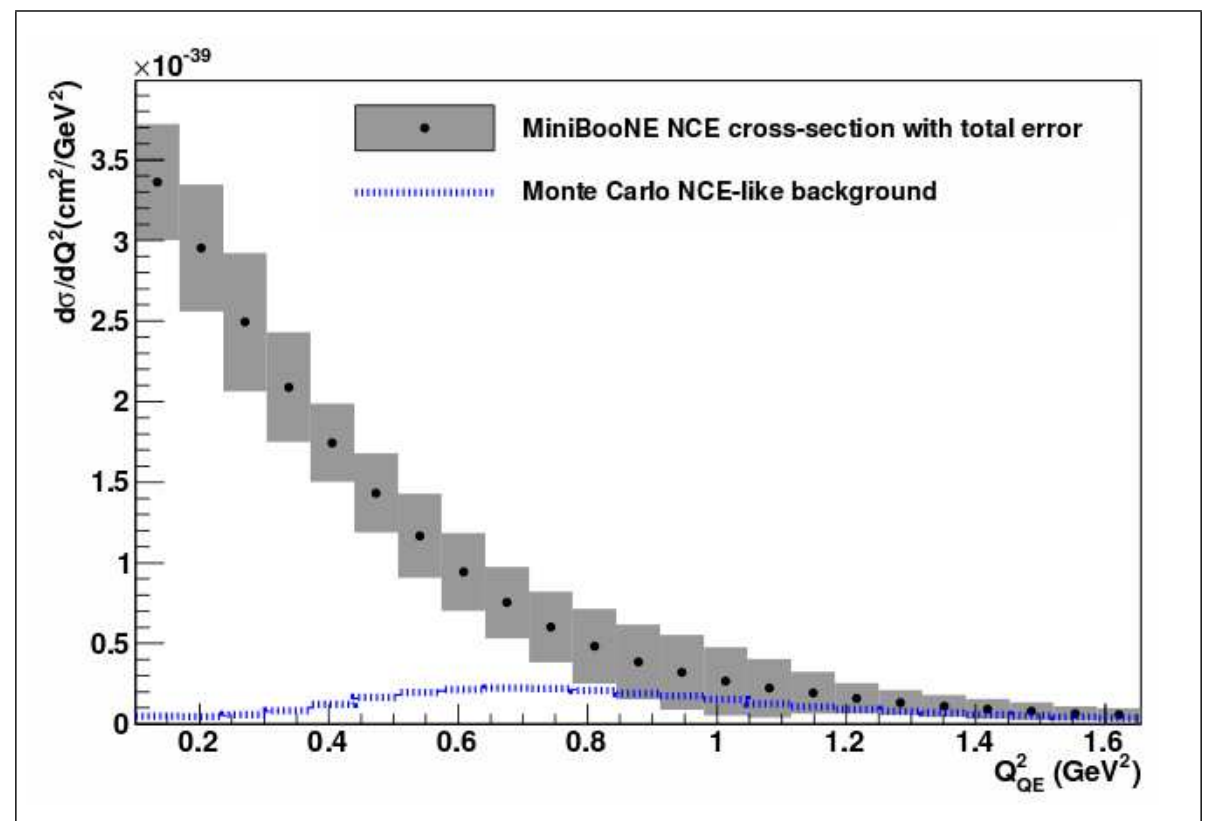

Figure 2.8 MiniBooNE Flux-averaged Neutral Current Differential Cross Section. The blue line is the predicted spectrum of elastic neutral current background which has been subtracted out from the total differential cross section [61].

C. H. Llewellyn-Smith [62] showed that the semileptonic neutral and charged currents ${ }^{7}$ cross sections are related ${ }^{8}$ through $\sin \theta_{W}$ by:

$$
R^{\nu}=\frac{\sigma_{\mathrm{NC}}}{\sigma_{\mathrm{CC}}}=\left[\frac{1}{2}-\sin ^{2} \theta_{W}+\frac{5}{9} \sin ^{4} \theta_{W}\right](1+r)
$$

where $r$ is the ratio of the antineutrino charge current cross section to the neutrino one. The CHARM collaboration [63] measured this ratio for energies larger than $2 \mathrm{GeV}$ to be:

$$
R^{\nu}=0.317 \pm 0.006
$$

${ }^{7}$ Charged current QE interactions.

${ }^{8}$ See equation (A.22). 


\section{$2.2 \quad$ Neutrino Masses}

The formalism ${ }^{9}$ by which particles acquire their mass in the Standard Model requires both left-handed ${ }^{10}$ and right-handed ${ }^{11}$ states for a given particle to have a mass term in the Lagrangian. Neutrinos are a special case since there is no evidence that supports the existence of right-handed neutrinos. Yet, there is no argument or symmetry within the Standard Model that explicitly forbids the existence of such neutrinos. In the case of the photon ${ }^{12}$, the gauge symmetry requires it to be massless.

In view of this, there are two possible mass terms: the Dirac term ${ }^{13}$ :

$$
m_{D}\left(\bar{\psi}_{R} \psi_{L}+\text { h.c. }\right)
$$

and the Majorana term:

$$
m_{M}\left(\bar{\psi}_{L}^{c} \psi_{L}+\text { h.c. }\right)
$$

where $\psi^{c}=C \gamma^{0} \psi^{*}$ is the charge conjugated field of $\psi$, and $\psi_{L}^{c} \equiv\left(1+\gamma_{5}\right) \psi^{c} / 2$ is right-handed. The Majorana term in equation (2.26) violates lepton number conservation by two units $^{14}$ and makes neutrinos indistinguishable from antineutrinos [64]. All the massive fundamental particles in the Standard Model have some type of charge (even though gluons are massless, these are linear combinations of color states), which changes sign under charge conjugation

\footnotetext{
${ }^{9}$ See appendix A for a brief summary of the formalism.

${ }^{10}$ See equation (A.2).

${ }^{11}$ See equation (A.3).

${ }^{12}$ See equation (A.19).

${ }^{13}$ See equation (A.20).

${ }^{14}$ In the Dirac mass term, the matter field has lepton number: +1 , and the antimatter field has lepton number: -1 . In the Majorana mass term, both fields have lepton number: +1 .
} 
providing a clear distinction between particles and antiparticles. A Majorana mass term is therefore only possible for neutral particles, exhibiting no charges of any kind; a feature that only neutrinos exhibit.

\subsubsection{Seesaw Mechanism}

The seesaw mechanism [65] is the simplest renormalizable model that introduces a mass term for neutrinos in the Lagrangian:

$$
\begin{aligned}
\mathcal{L}_{M} & =m_{D} \bar{\psi}_{R} \psi_{L}+\frac{m_{M}}{2} \bar{\psi}_{R}^{c} \psi_{R}+\text { h.c.. } \\
& =\frac{1}{2}\left(\bar{\psi}_{L}^{c} \psi_{R}\right)\left(\begin{array}{cc}
0 & m_{D} \\
m_{D} & m_{M}
\end{array}\right)\left(\begin{array}{c}
\psi_{L} \\
\psi_{R}^{c}
\end{array}\right)+\text { h.c. }
\end{aligned}
$$

The Lagrangian in equation (2.27) is simplified by diagonalizing its mass matrix. The diagonalization procedure defines a new two component field $\nu_{M}$, written as:

$$
\nu_{M} \equiv \psi_{L}+\psi_{L}^{c} \equiv\left(\begin{array}{c}
\nu_{\mathrm{sm}} \\
\nu_{\mathrm{nsm}}
\end{array}\right),
$$

such that:

$$
\mathcal{L}_{M}=\frac{1}{2}\left(\bar{\nu}_{\mathrm{sm}} \bar{\nu}_{\mathrm{nsm}}\right)\left(\begin{array}{cc}
m_{\mathrm{sm}} & 0 \\
0 & m_{\mathrm{nsm}}
\end{array}\right)\left(\begin{array}{c}
\nu_{\mathrm{sm}} \\
\nu_{\mathrm{nsm}}
\end{array}\right) .
$$

The $m_{D}$ scale is in the order of a typical Standard Model fermion, and $m_{M} \gg m_{D}$. The masses of the $\nu_{M}^{i}$ are [66]:

$$
m_{\mathrm{sm}} \cong \frac{m_{D}^{2}}{m_{M}}, \quad m_{\mathrm{nsm}} \cong m_{M} .
$$


The $\nu_{\mathrm{sm}}$ states are associated with the neutrinos of the Standard Model ${ }^{15}$, and the $\nu_{\mathrm{nsm}}$ states represent very massive neutrinos yet to be observed. Equation (2.30) suggests that the $m_{\mathrm{sm}}$ are very small, and the model suggests that the $m_{\mathrm{nsm}}$ are about $10^{15} \mathrm{GeV} / c^{2}$ [66]. The direct laboratory limits on the $m_{\mathrm{sm}}$ establish an upper limit in the range: $(0.3-$ 0.9) $\mathrm{eV} / \mathrm{c}^{2}$, depending on the nuclear model considered [67]. The most relevant feature of the seesaw mechanism is that it explains the lightness of the known neutrinos, the $\nu_{\mathrm{sm}}$ states, by setting a very high mass to the yet to-be-observed heavy neutrinos, the $\nu_{\mathrm{nsm}}$ states. The out-of-thermal-equilibrium decays of these heavy neutrinos are key to the attempts for explaining the baryon-antibaryon asymmetry of the Universe in terms of leptogenesis [66].

The leptonic part of the Lagrangian can be written in the mass basis of the charged leptons and of the singlet fermions as follows ${ }^{16}$ [68]:

$$
\mathcal{L}_{s s}=\mathbf{h}^{*}\left(\bar{L} \phi^{c *}\right) l_{R}-\boldsymbol{\lambda}^{*}\left(\bar{L} \phi^{*}\right) \nu_{\mathrm{nsm}}-\frac{1}{2} m_{\mathrm{nsm}} \bar{\nu}_{\mathrm{nsm}} \nu_{\mathrm{nsm}}^{c}+\text { h.c.. }
$$

with:

$$
\left(\bar{L} \phi^{c *}\right)=\left(\bar{\nu}_{L} \bar{l}_{L}\right)\left(\begin{array}{cc}
0 & -1 \\
1 & 0
\end{array}\right)\left(\begin{array}{c}
\phi^{-} \\
\phi^{* 0}
\end{array}\right)
$$

and where $\mathbf{h}$ and $\boldsymbol{\lambda}$ are matrices of Yukawa couplings. From the second Yukawa term in equation (2.31), the lightest right-handed heavy neutrino $\left(\nu_{\mathrm{nsm}}^{1}\right)$ can decay into:

$$
\nu_{\mathrm{nsm}}^{1} \rightarrow \phi l_{L}
$$

In the early Universe, when the temperature $\left(T_{U}\right)$ was $T_{U} \sim m_{M}$, the $\nu_{\text {nsm }}^{1}$ population was

${ }^{15}$ There are three neutrino eigenstates associated with each of the two states in equation (2.28).

${ }^{16}$ See equations (A.1, A.2, and A.3). 
stable. Once $T_{U}<m_{M}$ the population of $\nu_{\text {nsm }}^{1}$ is no longer stable, since they can not be produced any more, and the $\nu_{\mathrm{nsm}}^{1}$ all decayed. If there is an asymmetry ${ }^{17}(A)$ in the decay of the $\nu_{\text {nsm }}^{1}$ that favors the production of charged leptons over charged antileptons, there would be an excess of charged leptons in the early Universe. After charged leptons annihilation, such excess would be the origin of the matter-dominated Universe seen nowadays. $A$ is defined in terms of the decay rates as follows [68]:

$$
A_{k k} \equiv \frac{\Gamma\left(\nu_{\mathrm{nsm}}^{1} \rightarrow \phi l_{L}^{k}\right)-\Gamma\left(\nu_{\mathrm{nsm}}^{1} \rightarrow \bar{\phi} \bar{l}_{L}^{k}\right)}{\Gamma\left(\nu_{\mathrm{nsm}}^{1} \rightarrow \phi l_{L}\right)+\Gamma\left(\nu_{\mathrm{nsm}}^{1} \rightarrow \bar{\phi} \bar{l}_{L}\right)},
$$

where $k$ denotes the various lepton flavors. By definition: $\left|A_{k k}\right| \leq 1$. Usually, it is much smaller than 1. To account for the observed baryon asymmetry, it is required that: $\left|A_{k k}\right|>10^{-7}[68]$

\section{$2.3 \quad$ Neutrino Oscillations}

The charged current, given by equation (2.4), introduced the concept of quark and lepton mixing. The leptonic part of the Lagrangian in equation (2.3) is:

$$
\mathcal{L}_{W}^{l}=\frac{g}{2} W_{\mu}^{+}\left(\bar{\nu}_{e} \gamma^{\mu} e+\bar{\nu}_{\mu} \gamma^{\mu} \mu+\bar{\nu}_{\tau} \gamma^{\mu} \tau\right)+\text { h.c. }
$$

Taking a basis in which the charged leptons' mass term is diagonal, the neutrino flavor eigenstates are a linear combination of the neutrino mass eigenstates:

$$
\nu_{L}^{\alpha}=U_{\alpha i} \nu_{L}^{\prime i}, \quad \nu_{R}^{\alpha}=T_{\alpha i} \nu_{R}^{\prime i}
$$

\footnotetext{
${ }^{17}$ If $A>0$.
} 
which leads to the mass term:

$$
\mathcal{L}_{M}=\bar{\nu}_{L}^{\prime i} T_{i \alpha}^{\dagger} m_{\alpha \beta} U_{\beta j} \nu_{L}^{\prime i}+\text { h.c. }
$$

with:

$$
T^{\dagger} m U=m_{\mathrm{diag}}
$$

where $U$ is the PMNS matrix [69] from equation (2.4).

Since neutrinos are only observed by their role in the weak interaction, all observed neutrinos are represented in the flavor basis; however, only mass eigenstates propagate through space and time. If the resolution of real experiments allowed the direct measurement of neutrino masses in individual processes, the oscillations would not be of scientific interest at all, i.e. once a neutrino is produced in a mass eigenstate it remains in that mass eigenstate forever. The current understanding of how neutrinos interact is through the weak interaction and its flavor eigenstates, thus neutrino oscillations are unavoidable, under these conditions. The weak interaction produces neutrinos in a given flavor eigenstate, and these are detected by means of a weak interaction process which involves a given flavor eigenstate. The question is: would those two states be the same?

In vacuum, a neutrino state of the generation $\alpha$, after a time interval $t$, is given by [64]:

$$
\left|\nu^{\alpha}\right\rangle_{t}=\sum_{i} U_{\alpha i} e^{-\imath E_{i} t}\left|\nu^{\prime i}\right\rangle
$$

and the transition amplitude to the state $\nu^{\beta}$ is:

$$
\left\langle\nu^{\beta} \mid \nu^{\alpha}\right\rangle_{t}=\sum U_{\alpha i} U_{i \beta}^{\dagger} e^{-\imath E_{i} t}
$$


The lightness of neutrinos yields $|\mathbf{p}| \gg m_{i}$, which means: $E_{i}=\sqrt{\mathbf{p}^{2}+m_{i}^{2}} \simeq \mathbf{p}+m_{i}^{2} / 2 E$. With this approximation, and using equation (2.36), equation (2.39) becomes:

$$
\begin{aligned}
& \left|\nu^{\alpha}\right\rangle_{t} \simeq e^{-\imath \mathbf{p} t} U\left(\begin{array}{lll}
e^{-\imath m_{1}^{2} t / 2 E} & & \\
& e^{-\imath m_{2}^{2} t / 2 E} & \\
& & e^{-\imath m_{3}^{2} t / 2 E}
\end{array}\right) U^{\dagger}\left|\nu^{\beta}\right\rangle \\
& =e^{-\imath \mathbf{p} t} U\left(\begin{array}{rrr}
1-\imath \frac{m_{1}^{2} t}{2 E}+\cdots & & \\
& 1-\imath \frac{m_{2}^{2} t}{2 E}+\cdots & \\
& & 1-\imath \frac{m_{3}^{2} t}{2 E}+\cdots
\end{array}\right) U^{\dagger}\left|\nu^{\beta}\right\rangle .
\end{aligned}
$$

Using equation (2.38) as:

$$
U^{\dagger} m^{\dagger} m U=m_{\operatorname{diag}}^{2}=\left(\begin{array}{ccc}
m_{1}^{2} & & 0 \\
& m_{2}^{2} & \\
0 & & m_{3}^{2}
\end{array}\right),
$$

equation (2.41) becomes:

$$
\left|\nu^{\alpha}\right\rangle_{t} \simeq e^{-\imath \mathbf{p} t}\left[e^{-\imath^{\frac{m^{\dagger} m}{2 E}} t}\right]_{\alpha \beta}\left|\nu^{\beta}\right\rangle
$$

Since $\mathrm{NO} \nu \mathrm{A}$ will study the oscillation of $\nu_{\mu}$ to $\nu_{e}$, it is interesting to see the mixing between two generations ${ }^{18}$, which is a valid approximation in this case. The relevant mixing matrix $(U)$ is [64]:

$$
U=\left(\begin{array}{cc}
\cos \theta & \sin \theta \\
-\sin \theta & \cos \theta
\end{array}\right) .
$$

\footnotetext{
${ }^{18}$ For the three generation mixing see e.g. [66].
} 
Using equation (2.44), $m^{\dagger} m$ can be written as:

$$
m^{\dagger} m=U m_{\operatorname{diag}}^{2} U^{\dagger}=\frac{m_{1}^{2}+m_{2}^{2}}{2}+\frac{\Delta m^{2}}{2}\left(\begin{array}{cc}
-\cos 2 \theta & \sin 2 \theta \\
\sin \theta & \cos 2 \theta
\end{array}\right)
$$

where $\Delta m^{2}=m_{2}^{2}-m_{1}^{2}$. Equation (2.45) allows to rewrite equation (2.43) as:

$$
\left|\nu^{\alpha}\right\rangle(t)=\left(\begin{array}{cc}
\cos \frac{\Delta m^{2}}{4 E} t-\imath \sin \frac{\Delta m^{2}}{4 E} t \cos 2 \theta & -\imath \sin \frac{\Delta m^{2}}{4 E} t \sin 2 \theta \\
-\imath \sin \frac{\Delta m^{2}}{4 E} t \sin 2 \theta & \cos \frac{\Delta m^{2}}{4 E} t+\imath \sin \frac{\Delta m^{2}}{4 E} t \cos 2 \theta
\end{array}\right)\left|\nu^{\beta}\right\rangle .
$$

The transition from $\left|\nu^{\mu}\right\rangle$ to $\left|\nu^{e}\right\rangle(t)$ is then given by:

$$
\begin{aligned}
\left\langle\nu^{\mu} \mid \nu^{e}\right\rangle(t) & \quad\left(\begin{array}{ll}
0 & 1
\end{array}\right)\left(\begin{array}{cc}
\cos \frac{\Delta m^{2}}{4 E} t-\imath \sin \frac{\Delta m^{2}}{4 E} t \cos 2 \theta & -\imath \sin \frac{\Delta m^{2}}{4 E} t \sin 2 \theta \\
-\imath \sin \frac{\Delta m^{2}}{4 E} t \sin 2 \theta & \cos \frac{\Delta m^{2}}{4 E} t+\imath \sin \frac{\Delta m^{2}}{4 E} t \cos 2 \theta
\end{array}\right)\left(\begin{array}{l}
0 \\
1
\end{array}\right) \\
= & -\imath \sin \frac{\Delta m^{2}}{4 E} t \sin 2 \theta .
\end{aligned}
$$

The physical meaning of the transition: $\left\langle\nu^{\mu} \mid \nu^{e}\right\rangle(t)$, is understood through the transition probability $\left(P_{\nu_{\mu} \rightarrow \nu_{e}}\right)$ expressed as:

$$
P_{\nu_{\mu} \rightarrow \nu_{e}}=\left|\left\langle\nu^{\mu} \mid \nu^{e}\right\rangle(t)\right|^{2}=\sin ^{2} \frac{\Delta m^{2}}{4 E} t \sin ^{2} 2 \theta
$$

Since neutrinos travel at nearly the speed of light, to a good approximation: $t \approx L / c$, where $L$ is the distance covered by the neutrino between the creation and detection points. With this small change, oscillation experiments might place their detectors at distances from the 
source that fulfill ${ }^{19}$ :

$$
\pi \leq \frac{\Delta m^{2} L}{2 E}
$$

The transition probability is maximal when:

$$
\pi=\frac{\Delta m^{2} L}{2 E}
$$

The ratio $L / E$ is of paramount importance in neutrino oscillation experiments since it determines much of the logistics of their experimental setup. All neutrino oscillation experiments that use a neutrino beam, send it through matter. Interactions with matter introduce changes in the formalism described in this section since $\nu_{e}$ interacts with electrons via charged and neutral current interactions, while $\nu_{\mu}$ and $\nu_{\tau}$ only interact with electrons via the neutral current interaction. As a consequence, there is a coherent effect in the transitions. See [64] for a formalism that describes neutrino oscillations in matter.

The formalism presented above is a simplification of the three neutrino oscillations. Nevertheless, this simplification is sufficiently good to allow the full problem to be represented as a collection of two neutrino transitions for which $U$ is:

$$
\begin{aligned}
U= & \left(\begin{array}{ccc}
1 & 0 & 0 \\
0 & c_{23} & s_{23} \\
0 & -s_{23} & c_{23}
\end{array}\right)\left(\begin{array}{ccc}
c_{13} & 0 & s_{13} e^{-\imath \delta} \\
0 & 1 & 0 \\
-s_{13} e^{\imath \delta} & 0 & c_{13}
\end{array}\right)\left(\begin{array}{ccc}
c_{12} & s_{12} & 0 \\
-s_{12} & c_{12} & 0 \\
0 & 0 & 1
\end{array}\right)\left(\begin{array}{ccc}
e^{\imath \frac{\varphi_{1}}{2}} & 0 & 0 \\
0 & e^{\imath \frac{\varphi_{2}}{2}} & 0 \\
0 & 0 & 1
\end{array}\right), \\
= & \left(\begin{array}{ccc}
c_{12} c_{13} e^{\imath \frac{\varphi_{1}}{2}} & s_{12} c_{13} e^{\imath \frac{\varphi_{2}}{2}} & s_{13} e^{-\imath \delta} \\
\left(-s_{12} c_{23}-c_{12} s_{23} s_{13} e^{\imath \delta}\right) e^{\imath \frac{\varphi_{1}}{2}} & \left(c_{12} c_{23}-s_{12} s_{23} s_{13} e^{\imath \delta}\right) e^{\imath \frac{\varphi_{2}}{2}} & s_{23} c_{13} \\
\left(s_{12} s_{23}-c_{12} c_{23} s_{13} e^{\imath \delta}\right) e^{\imath \frac{\varphi_{1}}{2}} & \left(-c_{12} s_{23}-s_{12} c_{23} s_{13} e^{\imath \delta}\right) e^{\imath \frac{\varphi_{2}}{2}} & c_{23} c_{13}
\end{array}\right) \cdot(2.51)
\end{aligned}
$$

\footnotetext{
${ }^{19}$ In natural units: $c=1=\hbar$.
} 
Here: $c_{i j} \equiv \cos \theta_{i j}$, and $s_{i j} \equiv \sin \theta_{i j} . \theta_{12}, \theta_{23}$, and $\theta_{13}$, are the mixing angles. The phase $\delta$ is the leptonic analogue of the single phase that appears in the quark mixing matrix, and $\varphi_{1}$, and $\varphi_{2}$ are Majorana phases that are relevant if neutrinos are their own antiparticles. The importance of the phases is evident when the Lagrangian in equation (2.35) is CP transformed:

$$
(C P) \mathcal{L}_{W}^{l}(C P)^{-1}=\frac{g}{2} W_{\mu}^{-} \bar{l}_{l} \gamma^{\mu} U^{*} \nu+\frac{g}{2} W_{\mu}^{+} \bar{\nu}_{l} \gamma^{\mu} U^{T} l
$$

If $U^{*} \neq U$, i.e. if $U$ is complex due to $\delta \neq 0$, the weak interaction is not CP invariant in the neutrino sector. The existence of phases is of great relevance to leptogenesis since these determine $^{20}$ whether there is an asymmetry in the production of leptons and antileptons in the early Universe [66].

In the three neutrino oscillations scenario, the parameter $\theta_{13}$ has an important role in the transition probability $\left(P_{\nu_{\mu} \rightarrow \nu_{e}}\right)$ presented in equation (2.48), which is the probability in vacuum for the two neutrino case. Once the matter effects are taken into account, equation (2.48) becomes $^{21}$ :

$$
\begin{aligned}
P_{\nu \mu \rightarrow \nu e}^{\text {matter }} & \approx\left(1 \pm 2 \frac{E}{E_{R}}\right) P_{\nu_{\mu} \rightarrow \nu_{e}}^{\text {vacuum }} \\
& \approx\left(1 \pm 2 \frac{E}{E_{R}}\right)\left|2 U_{\mu 3}^{*} U_{e 3} C_{1}+2 U_{\mu 2}^{*} U_{e 2} C_{2}\right|^{2}
\end{aligned}
$$

where $E_{R}$ is the matter resonance energy associated with the atmospheric mass difference $\left(\Delta m_{32}^{2}\right)$ and the electron number density in Earth $\left(N_{e}\right)$ :

$$
E_{R}=\frac{\Delta m_{32}^{2}}{2 \sqrt{2} G_{F} N_{e}} .
$$

${ }^{20}$ The decay of right-handed neutrinos in the seesaw mechanism provides the asymmetry. ${ }^{21}$ The \pm in equation (2.53) is: + for neutrinos and - for antineutrinos. 


\begin{tabular}{|c|c|}
\hline Parameter & Value \\
\hline \hline $\sin ^{2} \theta_{13}$ & $0.0219_{-0.0011}^{+0.0010}$ \\
$\sin ^{2} \theta_{23}$ & $0.451 \pm 0.001 \oplus 0.557_{-0.035}^{+0.027}$ \\
$\sin ^{2} \theta_{12}$ & $0.304 \pm 0.012$ \\
$\Delta m_{31}^{2}$ & $(2.458 \pm 0.002) \times 10^{-3} \mathrm{eV}^{2}$ \\
$\Delta m_{32}^{2}$ & $(-2.488 \pm 0.047) \times 10^{-3} \mathrm{eV}^{2}$ \\
$\Delta m_{21}^{2}$ & $\left(7.50_{-0.17}^{+0.19}\right) \times 10^{-5} \mathrm{eV}^{2}$ \\
\hline
\end{tabular}

Table 2.1 Neutrino Oscillation Parameters. Best measurements of the neutrino oscillation parameters [70].

$C_{1}$ and $C_{2}$ are oscillation parameters not relevant at the moment. From the transition matrix in equation (2.51), $P_{\nu_{\mu} \rightarrow \nu_{e}}^{\mathrm{vacuum}}$ depends on:

$$
2 U_{\mu 3}^{*} U_{e 3}=e^{-\imath \delta} \sin 2 \theta_{13} \sin \theta_{23}
$$

The experimental results obtained by Double Chooz, Daya Bay, and RENO measuring a non-zero $\theta_{13}$ allow the possibility for $\mathrm{CP}$ violation in the neutrino sector since the transition probability presented in equation (2.53) depends on the phase $\delta$. The transition probability $\bar{P}_{\bar{\nu} \mu \rightarrow \bar{\nu}_{e}}^{\mathrm{vacuum}}$ changes the sign of $\delta$, which directly affects the measurement of the asymmetry $(\tilde{A}):$

$$
\tilde{A}=\left|\frac{P-\bar{P}}{P+\bar{P}}\right|
$$

If it is non-zero, $\delta$ is non-zero, resulting in the mentioned $\mathrm{CP}$ violation. Both $\tilde{A}$ and $A_{k k}$ (recall equation (2.34)) are influenced by the phases $\delta$ and $\varphi$. A CP violation in the light neutrino sector could have $\mathrm{CP}$ violation implications in the heavy neutrino sector, which is the key to leptogenesis, as mentioned in section 2.2.1.

All the free parameters in the neutrino oscillation formalism have been measured [70], except for the phases. Table 2.1 summarizes the most accurate measurements of these 
parameters. Two open questions concerning these parameters remain unanswered, and are the subject of study of the next generation of neutrino oscillation experiments, including $\mathrm{NO} \nu \mathrm{A}$. The first is related to the octant of $\theta_{23}$, since this is measured through the expression $\sin ^{2} 2 \theta_{23}$, its measured value only shows that it is close to $\pi / 4$. However, whether $\theta_{23}$ is smaller or greater than $\pi / 4$ remains uncertain. The possibility for $\theta_{23}=\pi / 4$ implies that $\nu_{3}$ would have exactly the same amount of $\nu_{\mu}$ and $\nu_{\tau}$. The second question concerns the mass ordering, or mass hierarchy, of the mass eigenstates. Oscillation measurements are related to $\sin ^{2} \Delta m^{2} / 4 E$ (see equation (2.48)), where the sign of the $\Delta m^{2}$ is undetermined. The current knowledge is that there are two quasi-degenerate mass eigenstates, $m_{1}$ and $m_{2}$, and a third one, $m_{3}$, that is either much heavier (normal hierarchy), or much lighter (inverted hierarchy) than the other two, as shown in figure 2.9. In the normal hierarchy scenario, neutrino oscillations are enhanced and the antineutrino oscillations are attenuated, for the inverted hierarchy the opposite is true.

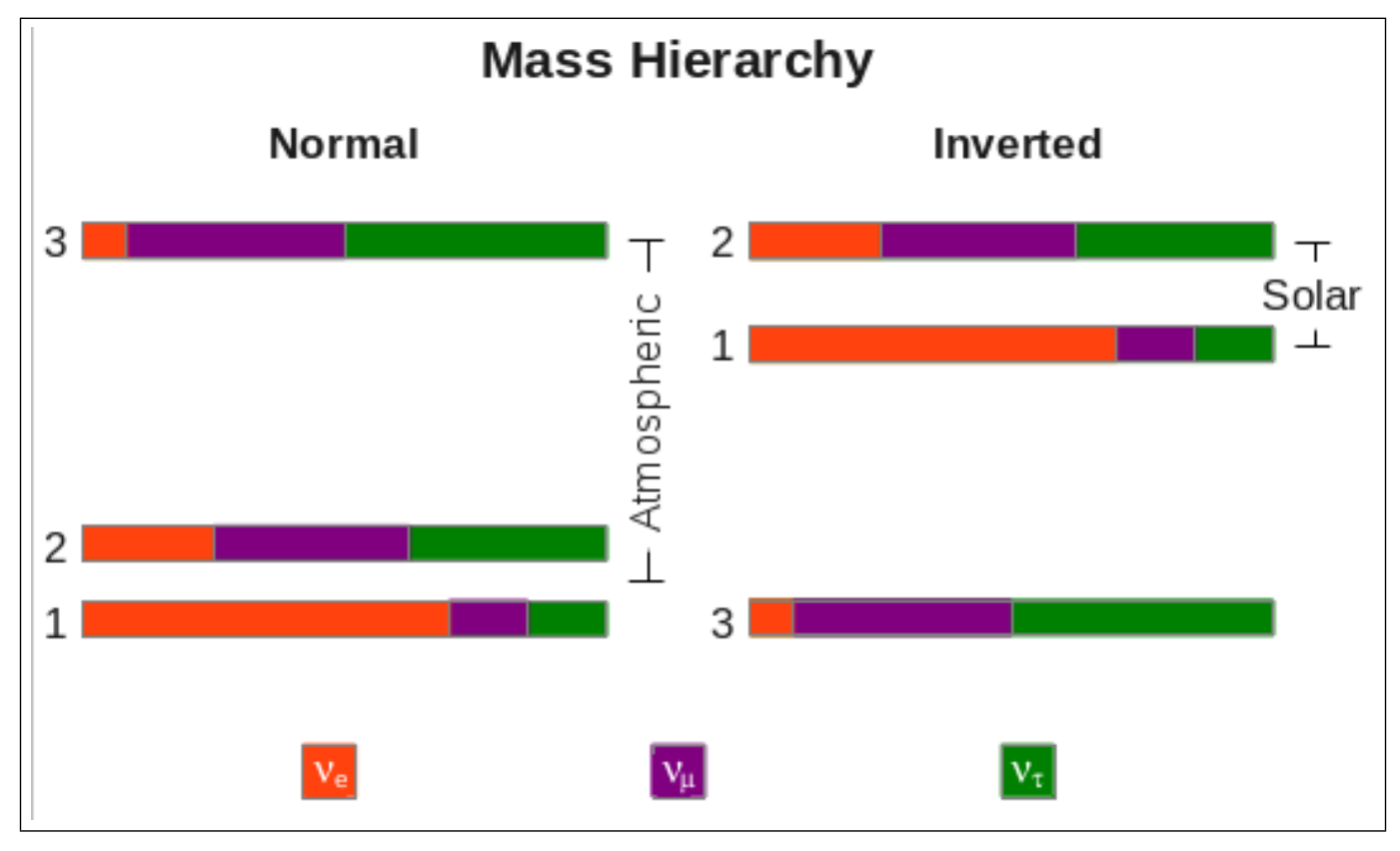

Figure 2.9 Neutrino Mass Hierarchy. 


\section{Chapter 3}

\section{NuMI Beam Overview}

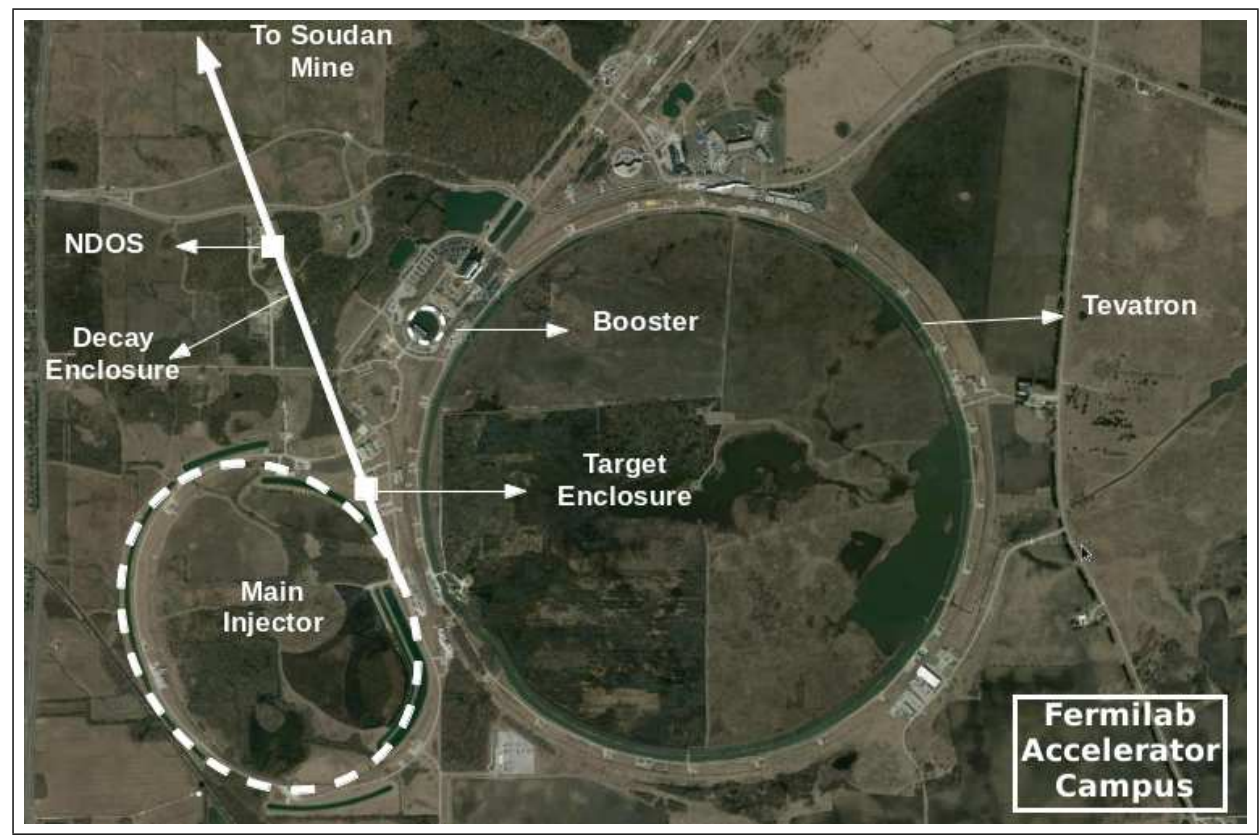

Figure 3.1 Fermilab Accelerator Campus. Picture from Google Maps.

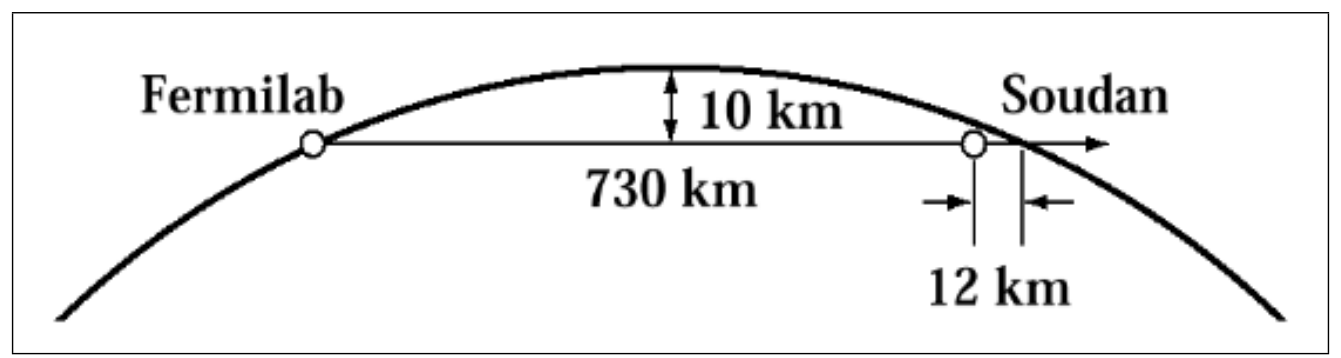

Figure 3.2 NuMI Beam Trajectory. Taken from [71].

The accelerator complex at Fermilab is currently dedicated to providing protons from the Main Injector (MI) to seed the neutrino beam that is used by the $\mathrm{NO} \nu \mathrm{A}$ experiment for 
their neutrino oscillation investigations, and used by other experiments that study neutrino interactions. In the next few years, accelerator-based neutrino experiments may resolve the neutrino mass hierarchy, the octant ambiguity in $\theta_{23}$, and begin to see hints that there is a large violation of the CP symmetry in neutrino oscillations.

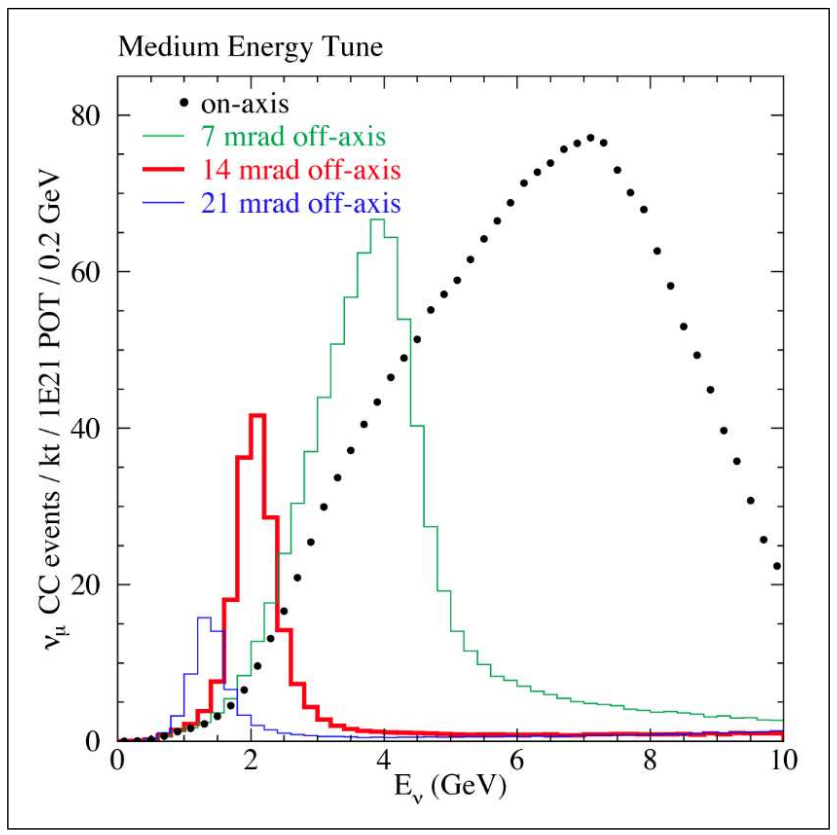

Figure 3.3 NuMI Beam Energy Spectrum. Neutrino event rates as a function of neutrino energy and off-axis angle [72].

The MI [73] is a very high intensity ${ }^{1}$ proton accelerator located at Fermilab (see figure 3.1). The MI provides $120 \mathrm{GeV}$ protons that serve as input to the Neutrinos at the Main Injector (NuMI) beam, which has a power ${ }^{2}$ of $320 \mathrm{~kW}$. This beam travels from the Fermilab Accelerator Campus, through the Earth, to the MI Neutrino Oscillation Search (MINOS) underground laboratory at Soudan, Minnesota (see figure 3.2). The energy spectrum of the beam, shown in figure 3.3, allows experiments like $\mathrm{NO} \nu \mathrm{A}$ to locate their detectors off the

\footnotetext{
${ }^{1}$ The number of protons per pulse is on the order of $10^{13}[72]$.

${ }^{2}$ The power cited is the one delivered to the $\mathrm{NO} \nu \mathrm{A}$ prototype detector. The MI is currently in the middle of an upgrade to deliver $700 \mathrm{~kW}$.
} 
beam axis (see figure 3.4) to obtain narrower neutrino energy spectra.

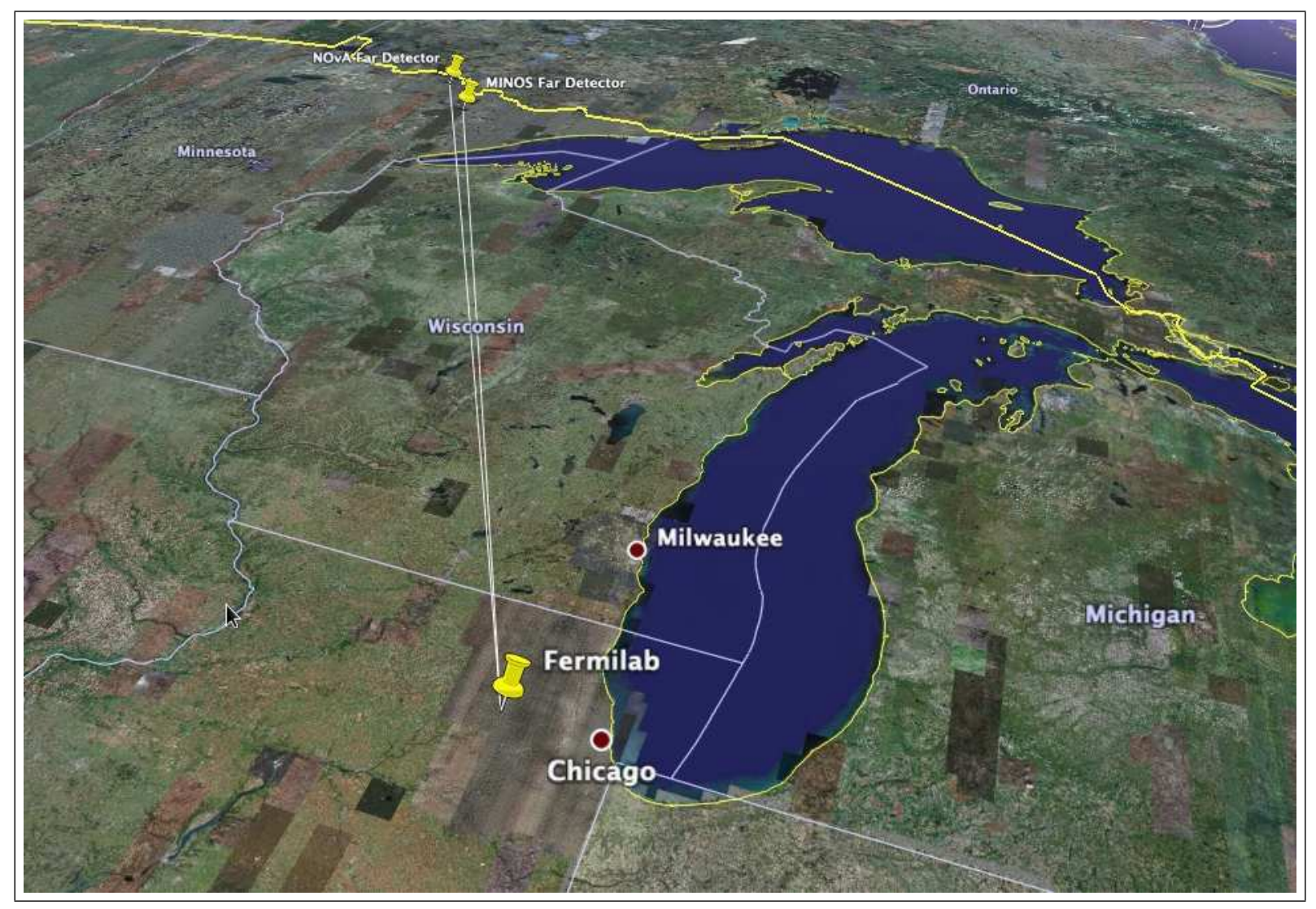

Figure 3.4 Location Of The $\mathbf{N O} \nu \mathbf{A}$ And MINOS Far Detectors. The MINOS far detector in Minnesota is on-axis. Picture from Google Earth.

Protons from the MI are directed every $1.9 \mathrm{~s}$, by single-turn extraction, into the NuMI beamline. At $320 \mathrm{~kW}$, there are $2.5 \times 10^{13}$ protons delivered in spills of $8 \mu \mathrm{s}$, and focused onto a target, producing secondary mesons, i.e. charged pions and kaons. For the neutrino beam to be able to reach the Soudan MINOS far detector site, the proton beam is directed downward at $58 \mathrm{mrad}$ before it strikes the $0.95 \mathrm{~m}$ long NuMI graphite target, as shown in figure 3.5. The forward-going mesons are focused and allowed to decay, producing the desired neutrino beam. The focusing is performed by a set of two parabolic magnetic horns. The charged pions and kaons selected by the horns propagate down a $675 \mathrm{~m}$ long ( $1 \mathrm{~m}$ radius) decay tunnel. A hadron absorber (beam stop) is placed at the end of the decay tunnel 
to remove the residual flux of protons and mesons, followed by a set of muon monitoring detectors, as shown in figure $3.6[71]$.

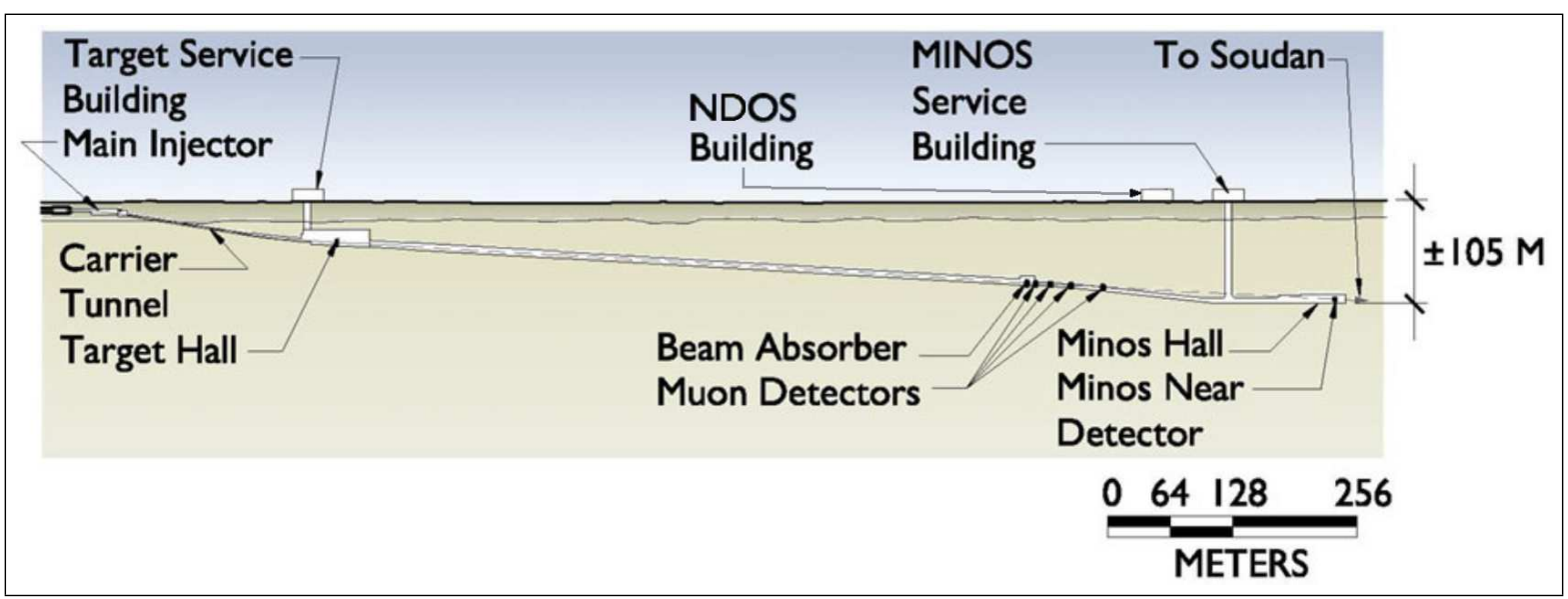

Figure 3.5 Schematics Of The NuMI Complex. Figure taken from [71].

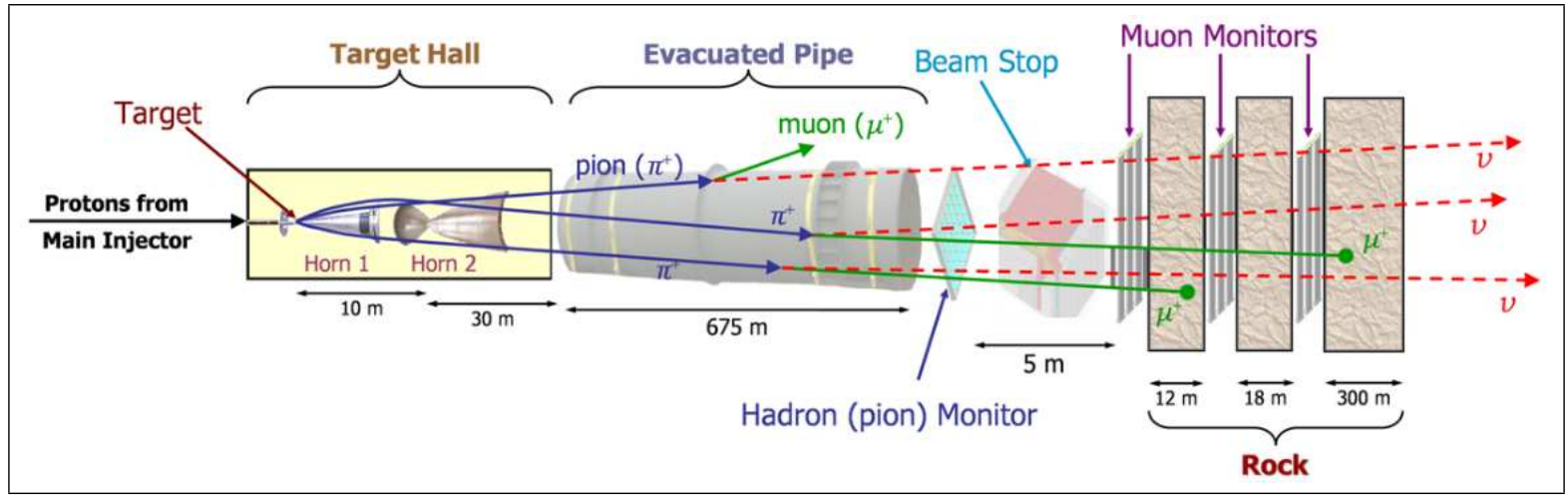

Figure 3.6 NuMI Beam Schematics. Taken from [74].

In neutrino oscillation searches, the flavor composition of the neutrino beam should be well known. The ideal case is to have a pure flavor beam, i.e. only one neutrino flavor. The NuMI beam design goals include: achieving the highest possible $\nu_{\mu}$ intensity, low backgrounds from other neutrino flavors, well understood spectra to control systematic uncertainties, and the selection of neutrino energy spectrum matched to the oscillation physics [75]. 


\subsection{Primary Beam System}

The process of producing the neutrino beam starts with a set of primary protons that hits a fixed target, which produces interactions that yield the mesons that decay to produce the neutrinos.

The primary protons are extracted from the MI ring and transferred through the extraction enclosure through a steeply inclined carrier pipe to the target region located about $50 \mathrm{~m}$ underground. The extraction mechanism is a horizontal kick, leading to Lambertson magnets ${ }^{3}$ deflecting the beam primarily in the vertical direction. The extracted protons are focused and bent strongly downward by a string of quadrupoles and bending magnets so that they enter the target hall located $122 \mathrm{~m}$ downstream of the extraction enclosure. Another set of bending magnets brings the protons to the correct pitch, $58 \mathrm{mrad}$, for a zero targeting angle beam directed toward the Soudan site [75].

To create the neutrino beam from the primary proton beam, these protons strike the NuMI target to produce hadrons. This target is sufficiently long to enable most of the primary protons to interact, as well as thin enough such that secondary interactions of the charged pions and kaons are minimized by allowing them to escape through the sides, as illustrated in figure 3.7. The depth of the field of the horn focusing system sets a limit on the length of the target. The desired flux of charged pions and kaons out of the target decreases with increasing radius due to particle re-absorption. The target stress due to the heat load of the proton beam also decreases with increasing radius. As a consequence of the last two statements, the target was designed to obtain the maximum yield and to ensure integrity against mechanical failure [75].

\footnotetext{
${ }^{3}$ Lambertson magnets are used to separate two adjacent beams by providing a bending field for one beam and a field-free region for the other beam [76].
} 


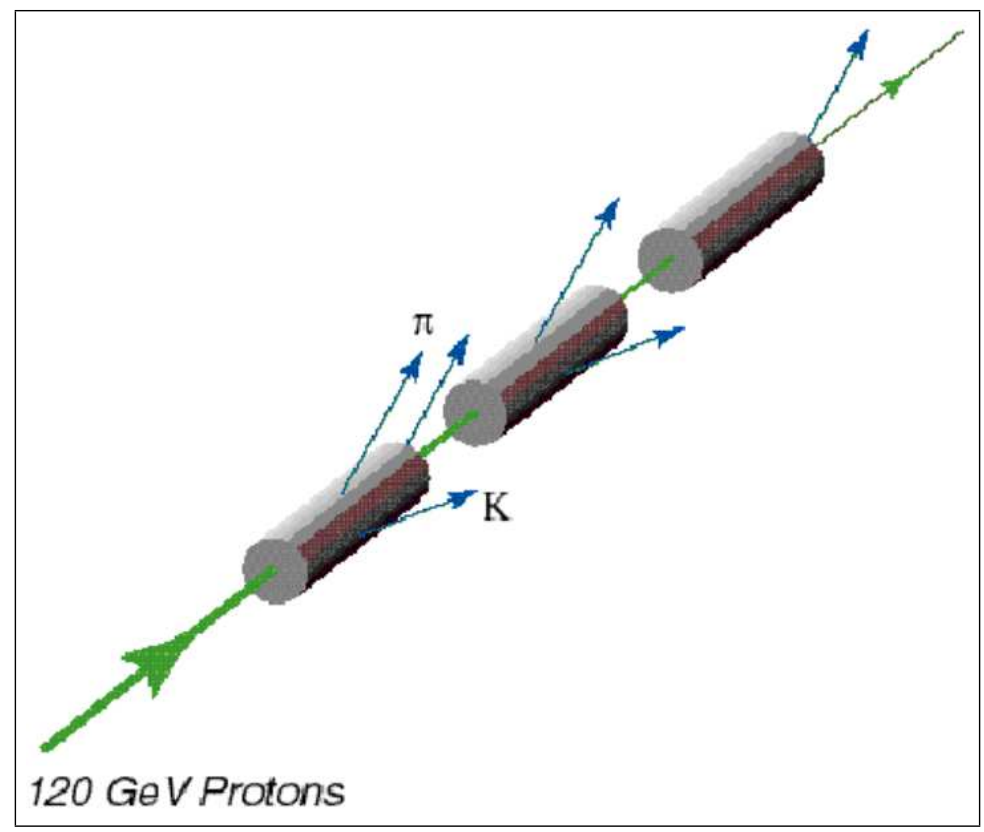

Figure 3.7 NuMI Target Drawing. Schematic drawing of a long and slim segmented target designed for optimum production and decay of charged pions and kaons [75].

\subsection{Secondary Beam System}

The second stage in the production of the neutrino beam is to focus the mesons coming out of the target in order to direct them toward the location of the detector. Therefore the focusing horns produce toroidal magnetic fields and act as lenses to bend the secondary particles back to the primary proton direction. The horns can either focus all the mesons at a particular momenta or some of the mesons at all momenta. The parabolic shaped horns produce magnetic fields that act as lenses, where the focal length is proportional to the momenta of the mesons. The selection of a particular target position causes particles of a certain momentum to be focused by the first horn. Mesons that were well focused by this horn pass unaffected through a central aperture in the second horn. Other mesons move to larger radii and are focused by the second horn, extending the momentum bite of the system, as illustrated in figure 3.6. The horns are designed for $205 \mathrm{kA}$ maximum current 
pulses of $5.2 \mathrm{~ms}$ half-sine wave width, with a repetition rate of $1.87 \mathrm{~s}$. The direction of the current flowing in the horns determines which mesons are focused depending on the sign of their electric charge. The forward horn current (FHC) focuses positive mesons which decay into neutrinos, and the reverse horn current (RHC) focuses negative mesons which decay into antineutrinos [75]. Figure 3.8 illustrates the direction of the two currents in the horns, as well as the beam direction through the horns.

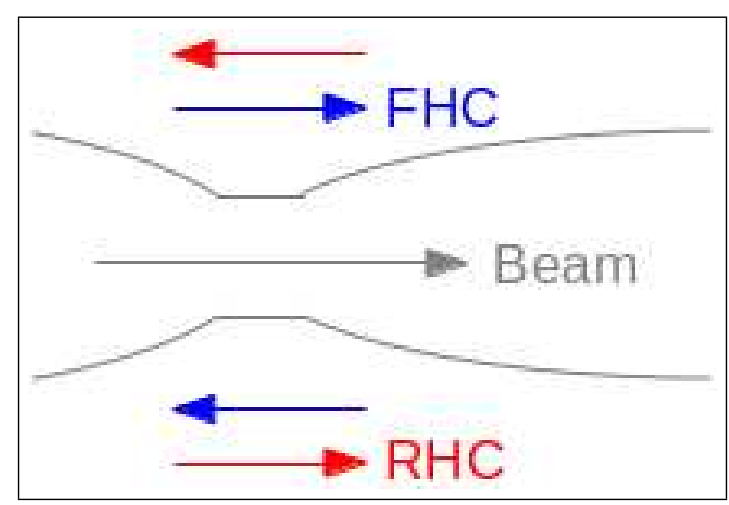

Figure 3.8 Beam And Electric Current Directions Through The NuMI Horns. The drawing illustrates the direction of the beam through the horn. The FHC (RHC) flows in the direction of the blue (red) arrows inside the conductors that make the horns.

The decay tunnel design allows for a sufficient flux of $\nu_{\mu}$ within the energy band required by MINOS and NO $\nu$ A. Alignment is paramount part of the tunnel's design. Misalignment of the decay tunnel along its length can not occlude the aperture of the tunnel by more than $2 \%$. The volume of the decay tunnel is carefully chosen to reach a compromise between the neutrino flux and the cost of construction ${ }^{4}$. The choice of tunnel's radius balanced the loss of secondary particles, that interact with the walls, with the cost of construction. A high vacuum inside the tunnel is necessary to prevent unwanted interactions with air molecules. The vacuum level in the tunnel ought to be 1 Torr or lower [75].

The main purpose of the hadron absorber is to eliminate hadrons that would overload the

\footnotetext{
${ }^{4}$ Pions of energies about $50 \mathrm{GeV}$ have a mean decay length of several $\mathrm{km}$.
} 
data acquisition systems of neutrino experiments on-site. All primary protons that did not interact with the target are absorbed at this stage. This absorber is not thick enough to stop the muons present in the beam, which are undesired background to the measurements of the neutrino experiments. These muons can be eliminated by providing sufficient material to absorb their energy. The NuMI beamline is located in dolomite, which is a dense rock. The $340 \mathrm{~m}$ of dolomite between the end of the hadron absorber and the MINOS hall is sufficient to stop all muons coming from the decay tunnel [75].

The neutrino beam monitoring systems enables the beam users to measure the quality of the neutrino beam. The neutrino flux is monitored through measuring: the spatial distribution of the hadrons directly upstream of the absorber, and the muons at various locations within the dolomite shield, as seen in figure 3.6. In order to detect variations, the muon intensity measurement is normalized to the number of incoming protons, and to each other, while the measured profiles are compared to nominal profiles [75].

\subsection{NuMI Beam Composition And Energy Spectrum}

\section{At $110 \mathrm{mrad}$}

Neutrinos are produced in many weak processes, including particle decays. For $\mathrm{NO} \nu \mathrm{A}$, the two most important sources of neutrinos are charged pion and kaon decays. The charged kaons are mesons made of two quarks: $K^{+}: u \bar{s}$, and $K^{-}: \bar{u} s$, with mean life

time: $\tau_{K}=(1.2380 \pm 0.0021) \times 10^{-8} \mathrm{~s}$, and mass: $m_{K}=(493.677 \pm 0.016) \mathrm{MeV} / c^{2}$ [77]. These have decay modes with only leptons (leptonic modes), with leptons and hadrons (semileptonic modes), and with only hadrons (hadronic modes). The modes with the largest 
branching ratios $\operatorname{are}^{5}[77]$ :

$$
\begin{aligned}
K^{ \pm} & \rightarrow \mu^{ \pm}+\nu_{\mu}\left(\bar{\nu}_{\mu}\right), \quad \text { leptonic mode, branching ratio: } 63.55 \pm 0.11 \% \\
& \rightarrow \pi^{ \pm}+\pi^{0}, \quad \text { hadronic mode, branching ratio: } 20.66 \pm 0.08 \% \\
& \rightarrow \pi^{0}+e^{ \pm}+\nu_{e}\left(\bar{\nu}_{e}\right), \quad \text { semileptonic mode, branching ratio: } 5.07 \pm 0.04 \% \\
& \rightarrow \pi^{0}+\mu^{ \pm}+\nu_{\mu}\left(\bar{\nu}_{\mu}\right), \quad \text { semileptonic mode, branching ratio: } 3.353 \pm 0.034 \%
\end{aligned}
$$

About $96 \%$ of all the $\nu_{\mu}$, from charged kaon decays, reaching the $\mathrm{NO} \nu \mathrm{A}$ prototype detector at $110 \mathrm{mrad}$ off the NuMI beam axis come from the leptonic mode. The rest of the $\nu_{\mu}$, at the prototype's location come from the semileptonic decay. A small $\nu_{e}$ component of the FHC of the NuMI beam comes from the semileptonic charged kaon decays. At the $\mathrm{NO} \nu \mathrm{A}$ far detector, the knowledge of this $\nu_{e}$ initial component of the beam is extremely important since it is the principal background to the $\nu_{e}$ appearance signal. There is no direct measurement of the charged kaons flux from the NuMI beam, therefore a measurement that can constrain the charged kaon production yield prediction could help to reduce the uncertainties in the $\nu_{e}$ flux at $14 \mathrm{mrad}$.

The charged pions contain two valence quarks: $\pi^{+}: u \bar{d}$, and $\pi^{-}: \bar{u} d$, with mean life time: $\tau_{\pi}=(2.6033 \pm 0.0005) \times 10^{-8} \mathrm{~s}$, and mass: $m_{\pi}=(139.570 \pm 0.00035) \mathrm{MeV} / c^{2}[78]$. The decay mode with the largest branching ratio is ${ }^{6}$ [78]:

$$
\pi^{ \pm} \rightarrow \mu^{ \pm}+\nu_{\mu}\left(\bar{\nu}_{\mu}\right), \quad \text { leptonic mode, branching ratio: } 99.98770 \pm 0.00004 \%
$$

The leptonic decay modes of charged kaons and pions are two body decays that produce

\footnotetext{
${ }^{5} \bar{\nu}_{\mu}\left(\bar{\nu}_{e}\right)$ are associated with $\mu^{-}\left(e^{-}\right)$and $K^{-}$.

${ }^{6} \bar{\nu}_{\mu}$ are associated with $\mu^{-}$and $\pi^{-}$.
} 


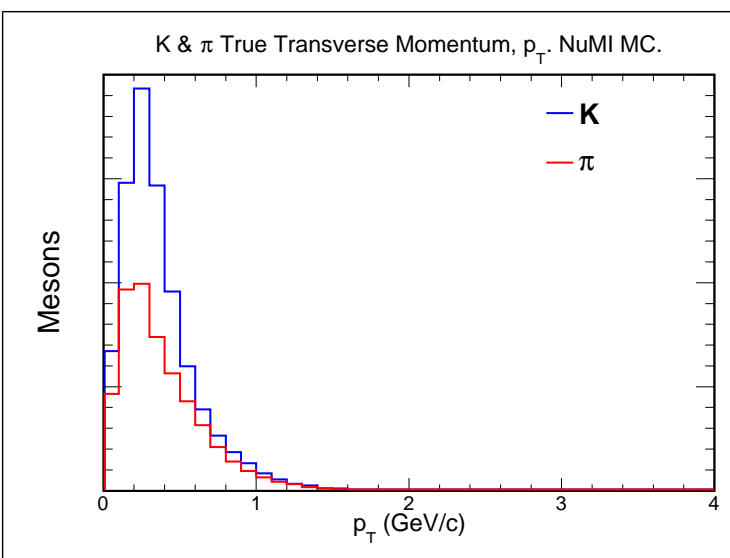

(a)

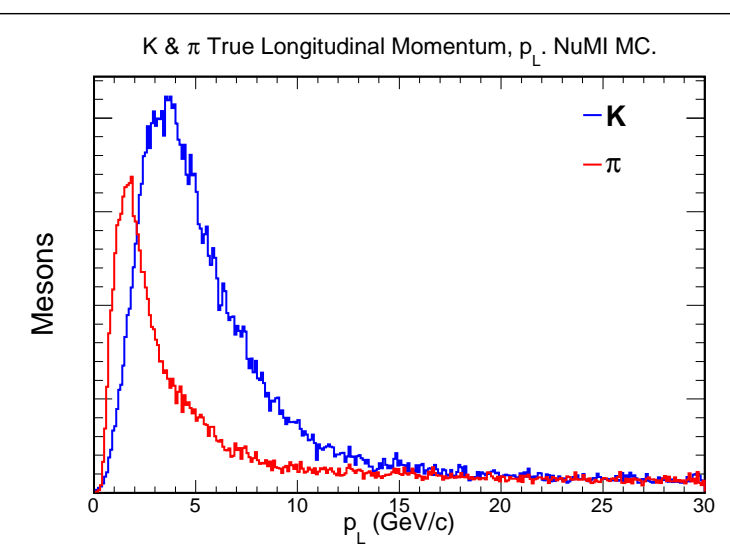

(b)

Figure 3.9 Meson Transverse And Longitudinal Momenta. Predicted (a) transverse and (b) longitudinal momentum distributions of charged kaons (blue) and pions (red) that decay into neutrinos detected in the $\mathrm{NO} \nu \mathrm{A}$ prototype detector. Momenta evaluated at the time of production of the meson in the target. MC simulation.

muons and neutrinos isotropically in the center of mass reference frame. In this reference frame the neutrino energies are fixed. The decaying meson is boosted to translate the decay into the laboratory reference frame. As a result, the neutrino energies will be relatively broad since these are now a function of the relativistic parameter $\gamma=E / m$. The neutrino energies in this case are also a function of the angle $\theta$ between the momentum of the decaying meson and the momentum of the neutrino. For small $\theta$, i.e. highly relativistic mesons, the flux and energy of the neutrinos are given by:

$$
\begin{aligned}
\Phi_{\nu} & =\left(\frac{2 \gamma}{1+\gamma^{2} \theta^{2}}\right)^{2} \frac{A}{4 \pi d^{2}} \\
E_{\nu} & =\frac{C_{m} E_{m}}{1+\gamma^{2} \theta^{2}}
\end{aligned}
$$

where $A$ is the transverse area of the detector that measures the neutrinos, $d$ is the distance between the decay point and the detector's location, $C_{m}$ is a constant that takes two values: one for charged kaons, $C_{K}=0.96$, and one for charged pions, $C_{\pi}=0.43$; and $E_{m}$ is the 
energy of the meson. Most of the mesons that yield the neutrinos seen at the location of the prototype detector have transverse momenta $\left(p_{T}\right)$ of the order of $300 \mathrm{MeV}$, as seen in figure 3.9a. The longitudinal momenta $\left(p_{L}\right)$ of the mesons is mostly below $10 \mathrm{GeV} / \mathrm{c}$, as seen in figure $3.9 \mathrm{~b}$.

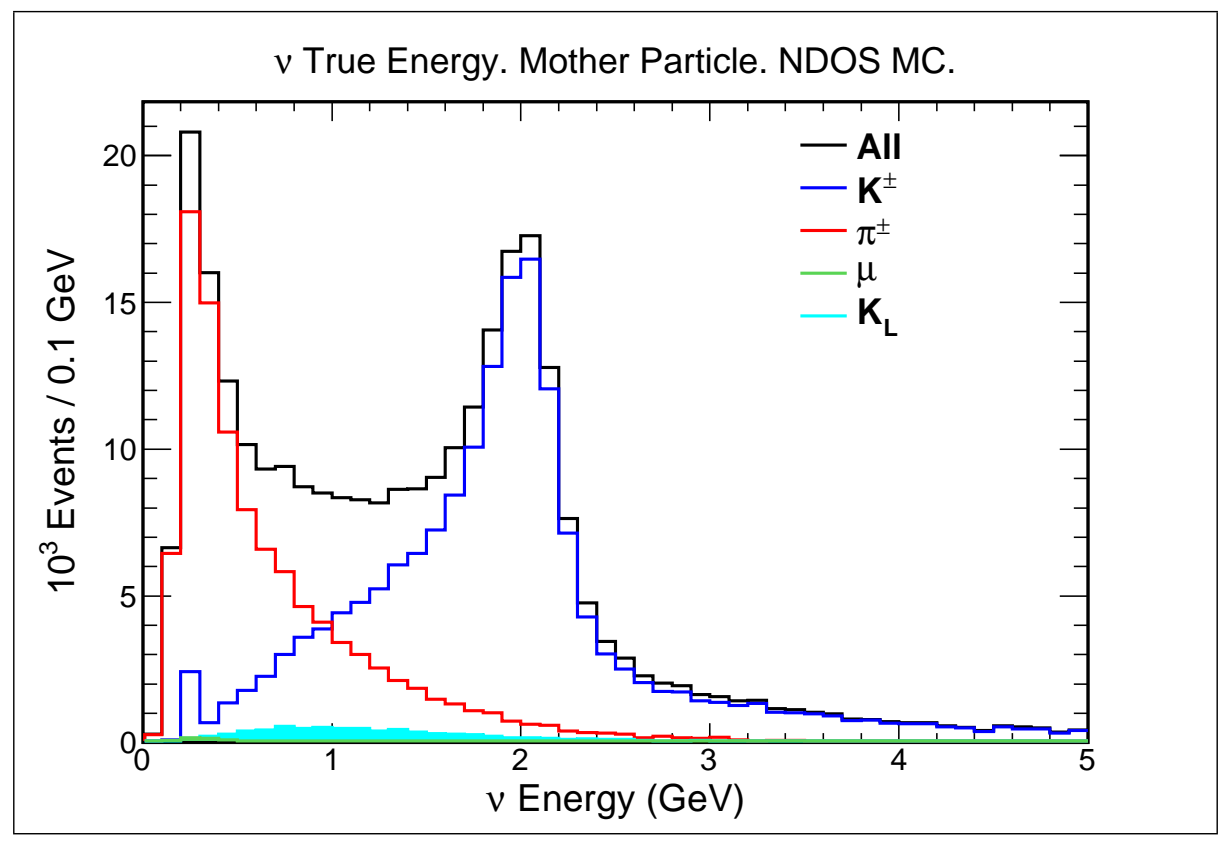

Figure 3.10 Neutrino Energy Distribution Discriminated By The Mother Particle Of The Neutrino. All mother particles (black), $\pi^{ \pm}$(red), $K^{ \pm}$(blue), $K_{L}$ (light blue), and $\mu^{ \pm}$(green) energy distributions. MC simulation.

The neutrino energy spectrum observed with the prototype detector shows two peaks: one comes from charged pion decays, around $0.2 \mathrm{GeV}$, and the second resulting from charged kaon decays, around $2 \mathrm{GeV}$. The spectrum is produced mostly by $\nu_{\mu}$. However this also receives contributions from $\bar{\nu}_{\mu}, \nu_{e}$, and $\bar{\nu}_{e}$ which arise from the semileptonic charged kaon decays, the leptonic decay of $K^{-}$that were not defocused by the horns, and muons and $K_{L}$ decays. $60.2 \%$ of all neutrinos come from charged kaon decays, $36.6 \%$ come from charged pion decays, $2.9 \%$ resulting from $K_{L}$ decays, and $0.2 \%$ arising from decayed muons, as shown in figure 3.10. The 4 neutrino components of the predicted energy spectrum, shown in figure 


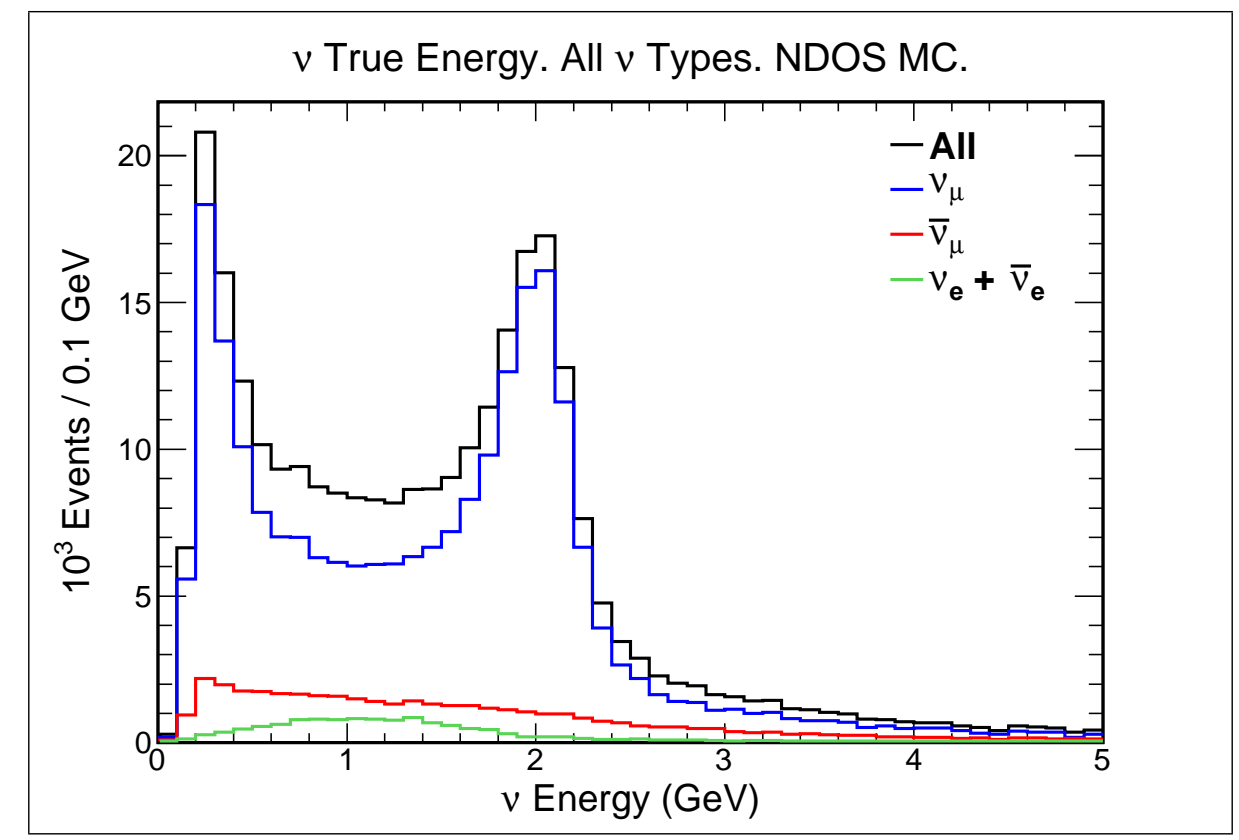

Figure 3.11 Neutrino Energy Distribution Discriminated By The Neutrino Type. All neutrino types (black), $\nu_{\mu}$ (blue), $\bar{\nu}_{\mu}$ (red), and $\nu_{e}+\bar{\nu}_{e}$ (green) energy distributions. MC simulation.

3.11, are distributed as follows: $\nu_{\mu} 81 \%, \bar{\nu}_{\mu} 14.3 \%, \nu_{e} 3.9 \%$, and $\bar{\nu}_{e} 0.8 \%$. The ratio $\frac{K}{\pi}$ as a function of energy is shown in figure 3.12. For energies higher than $1.8 \mathrm{GeV}$ the ratio remains above 10. This motivates a measurement of the neutrino flux coming from charged kaon decays due to the low background from neutrinos produced in other decays. 


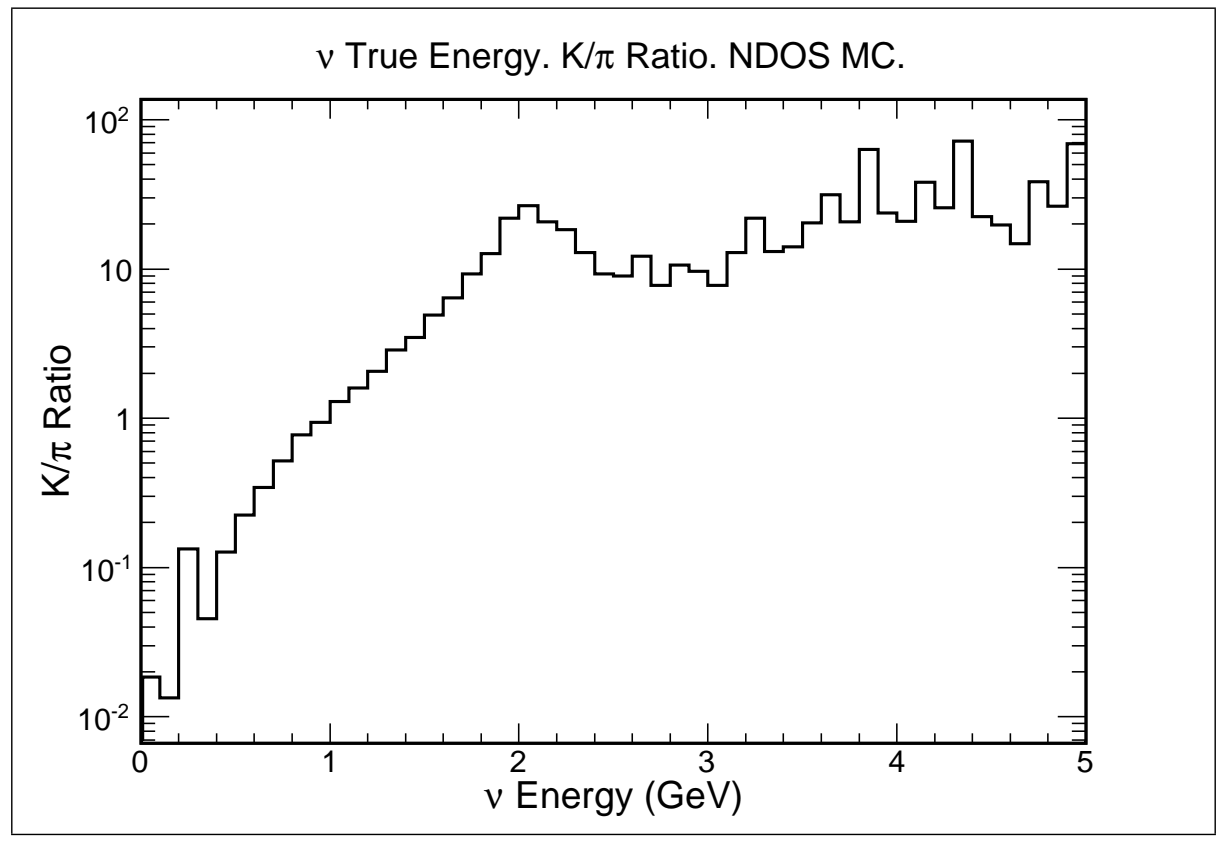

Figure 3.12 Ratio Of Neutrinos From Charged Kaon Decays Over Neutrinos From Charged Pion Decays. MC simulation. 


\section{Chapter 4}

\section{The NO $\nu$ A Prototype Detector}

\subsection{Experiment Overview}

The NO $\nu \mathrm{A}$ experiment will use the NuMI beam to measure $\nu_{\mu} \rightarrow \nu_{e}$ oscillations. The neutrino energies at the NuMI beam are a function of the energy of the meson, as well as of the angle between the momentum of the meson that produces the neutrino and momentum of the neutrino, as seen in equation (3.3). The MINOS experiment, located on the NuMI beam axis, measured neutrinos on a wide energy range [79] (see figure 3.3). The $\mathrm{NO} \nu \mathrm{A}$ experiment, 14 mrad off the NuMI beam axis, will measure neutrinos at a much narrowed energy band centered at $2 \mathrm{GeV}$. The far detector's location, $810 \mathrm{~km}$ away from the neutrino source (see figure 3.4), and the narrow neutrino energies observed there, produce the ideal conditions to observe the $\nu_{\mu} \rightarrow \nu_{e}$ oscillation very close to the maximum of probability. Using equation (2.49), this maximum occurs at:

$$
\frac{2 c \hbar \pi}{\Delta m_{32}^{2}}=\frac{L}{E} \approx 534 \frac{\mathrm{km}}{\mathrm{GeV}}
$$

while $\mathrm{NO} \nu \mathrm{A}$ has $L / E=505 \mathrm{~km} / \mathrm{GeV}$. Under such circumstances, $\mathrm{NO} \nu \mathrm{A}$ will be capable of determining the mass hierarchy and the $\mathrm{CP}$ violation with a significance up to $2 \sigma$, depending on the real value of the oscillation parameters ${ }^{1}$.

\footnotetext{
${ }^{1}$ A sample bi-probability point fulfilling the previous statement is shown in figure 4.1.
} 


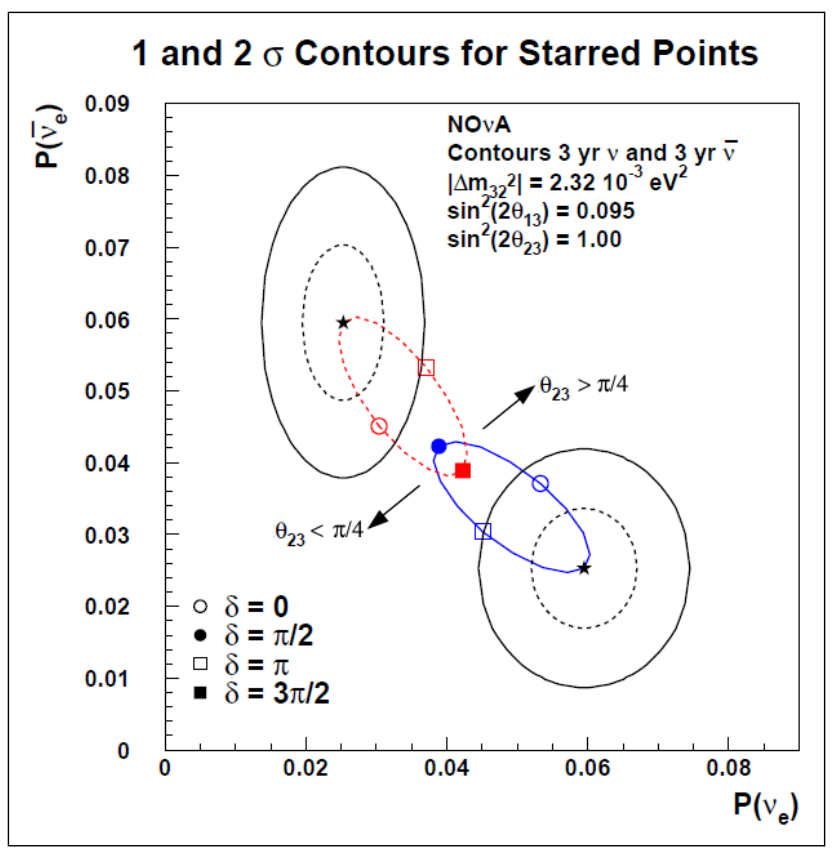

Figure 4.1 Bi-probability Plot. Four values of the phase $\delta$ are presented of each of the mass hierarchies: normal (blue) and inverted (red). Official NO $\nu$ A figure [81]. $1 \sigma$ and $2 \sigma$ contours are drawn around the estimated bi-probability points. The lower right point represented by a star is at $\delta=3 \pi / 2$. The upper left point represented by a star is at $\delta=\pi / 2$. MC simulation. 
$\mathrm{NO} \nu \mathrm{A}$ plans to collect three years of data in each mode ${ }^{2}$. From each mode, the transition probabilities (recall equation (2.53)): $P\left(\nu_{e}\right)$ for $\nu_{\mu} \rightarrow \nu_{e}$, and $P\left(\bar{\nu}_{e}\right)$ for $\bar{\nu}_{\mu} \rightarrow \bar{\nu}_{e}$, will be estimated. Once these two probabilities are calculated, a point can be drawn in a biprobability plot [80] like the one shown in figure 4.1, and the mass hierarchy and the phase $\delta$ could be resolved. $1 \sigma$ and $2 \sigma$ contours are shown around the calculated points. The ellipses are representations of the various possible values of $\delta$. The maximum CP violation occurs at $\delta=\pi / 2,3 \pi / 2$, while no $\mathrm{CP}$ violation occurs for $\delta=0, \pi$. Each mass hierarchy has its own ellipse due to matter effects. The bi-probability plots summarize all the oscillation parameters in an instructive fashion. Figure 4.1 assumes $\theta_{23}=\pi / 4$. Should $\theta_{23}>\pi / 4$, the ellipses will move towards higher $P\left(\nu_{e}\right)$ values along the $P\left(\nu_{e}\right)=P\left(\bar{\nu}_{e}\right)$ line. On the other hand, should $\theta_{23}<\pi / 4$, the ellipses will move downwards to lower $P\left(\nu_{e}\right)$ values along the $P\left(\nu_{e}\right)=P\left(\bar{\nu}_{e}\right)$ line. The best case scenario for $\mathrm{NO} \nu \mathrm{A}$ would be a normal hierarchy and $\delta=3 \pi / 2$, or an inverted hierarchy and $\delta=\pi / 2$. These two points will exclude the other mass hierarchy with a $2 \sigma$ significance.

The measured value of $\theta_{13}$ (see table 2.1) gives $\mathrm{NO} \nu \mathrm{A}$ the best sensitivity to: resolve the mass hierarchy, measure $\delta$, and resolve the octant of $\theta_{23}$. In order to make these measurements, $\mathrm{NO} \nu \mathrm{A}$ built two detectors. The near detector at Fermilab has a $3.9 \times 3.9 \mathrm{~m}^{2}$ transverse area, and is $14.3 \mathrm{~m}$ long. The far detector has a $15.6 \times 15.6 \mathrm{~m}^{2}$ transverse area, and is $63.0 \mathrm{~m}$ long. Figure 4.2 shows to scale the three detectors. An Airbus A380-800 is included in the figure for scale. The far detector is the largest free-standing PVC structure in the world.

\footnotetext{
${ }^{2}$ Neutrino and anti-neutrino modes.
} 


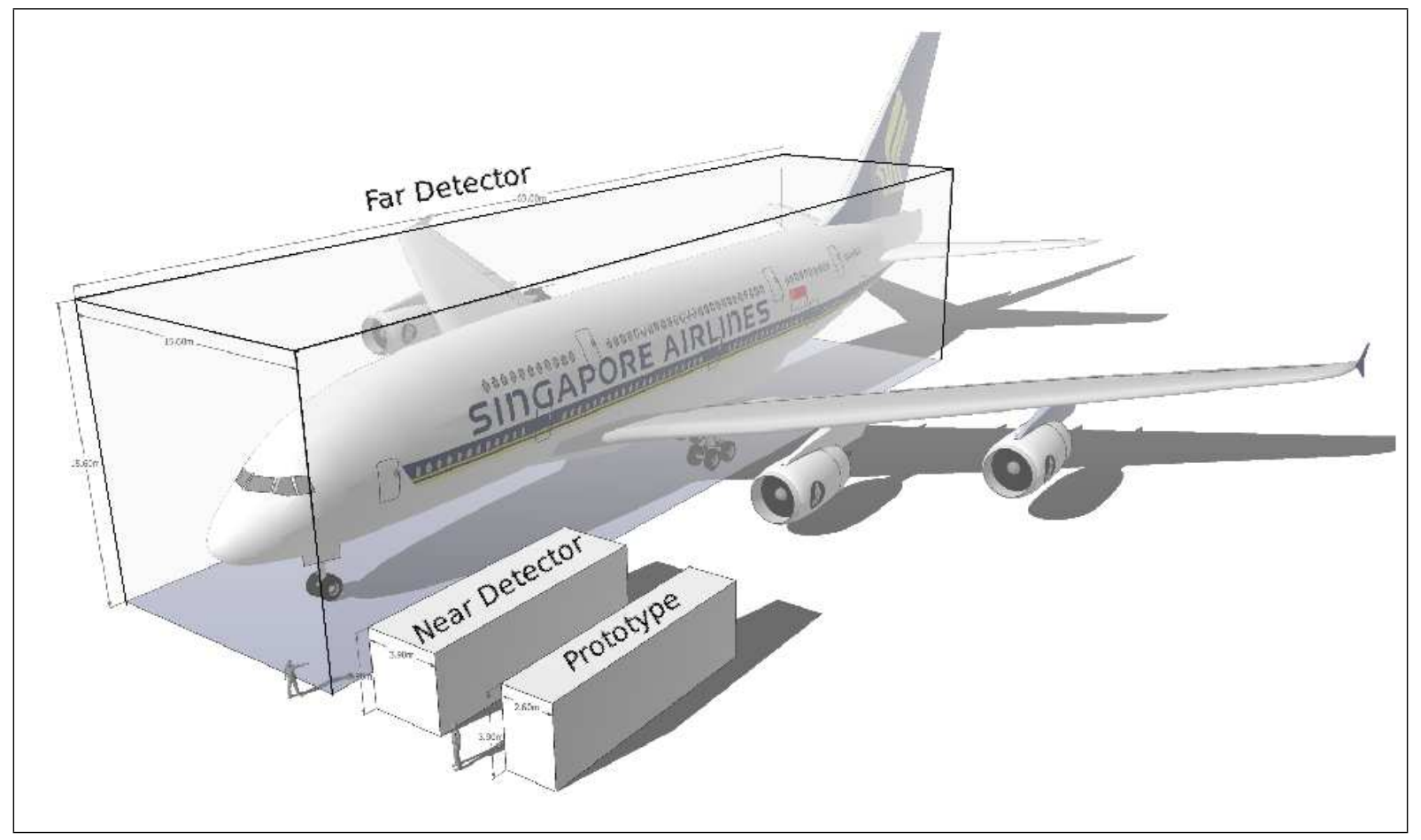

Figure 4.2 Drawing Of The NO $\nu$ A Detectors. NO $\nu$ A detectors drawn to scale. An average height person is shown to scale. An Airbus A380-800, drawn to scale, is included for illustration purposes [82]. 


\subsection{The Near Detector On The Surface}
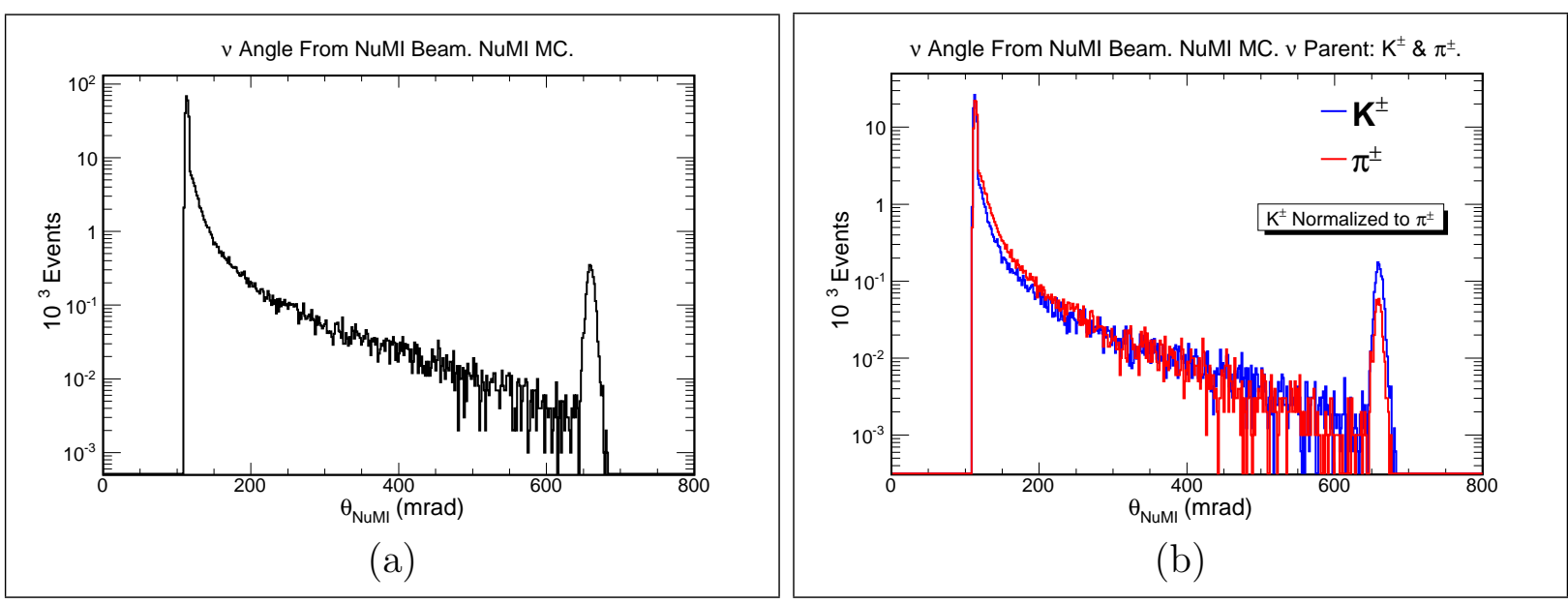

Figure 4.3 Angle Of Neutrinos With Respect To The NuMI Beam. (a) $\theta_{\text {NuMI }}$ for neutrinos from all parents. (b) $\theta_{\mathrm{NuMI}}$ for neutrinos from charged kaons (blue) and charged pions (red) parents. The charged kaons area is normalized to that of the charged pions. MC simulation.

The $\mathrm{NO} \nu \mathrm{A}$ prototype detector is located on the surface, north from the MI at Fermilab (see figure 3.1), and 110 mrad off the NuMI beam axis (see figure 3.5). This angle is measured from the NuMI target to the origin of coordinates of the prototype detector. Not all neutrinos are created at the same location along the decay pipe, their angular distribution is shown in figure $4.3 \mathrm{a}$ for all neutrino parents. About $77 \%$ of all these neutrinos have:

$$
110 \mathrm{mrad}<\theta_{\mathrm{NuMI}}<120 \mathrm{mrad}
$$

The angular distributions for neutrinos from charged kaon and pion decays are shown in figure 4.3b. Neutrinos from charged pion decays have a slightly higher rate between:

$$
120 \mathrm{mrad}<\theta_{\mathrm{NuMI}}<200 \mathrm{mrad}
$$


when compared with neutrinos from charged kaon decays. The peak of neutrinos around $650 \mathrm{mrad}$, in figure 4.3, comes predominantly from low longitudinal momentum mesons (about $75 \%$ of which are charged kaons decaying into a $\nu_{\mu}$ and a muon), as seen in figure 4.4a. The transverse momentum of these mesons is shown in figure 4.4b. Low energy neutrinos make this peak, as seen in figure 4.4c.

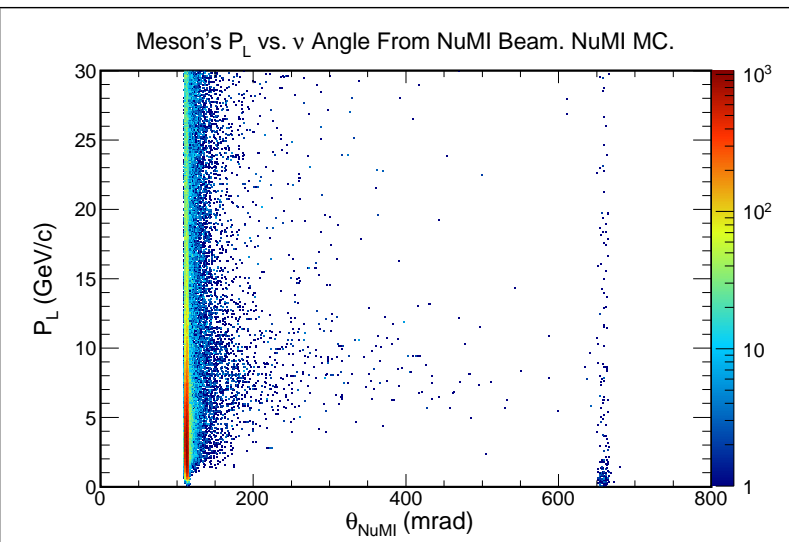

(a)

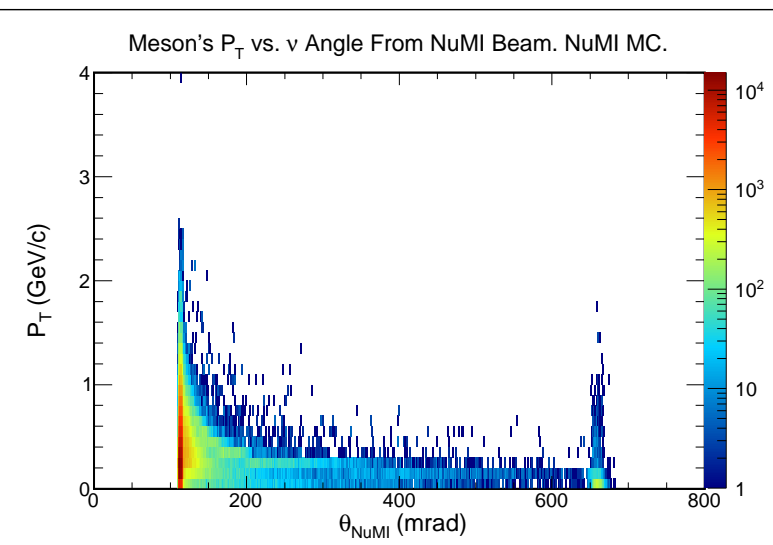

(b)

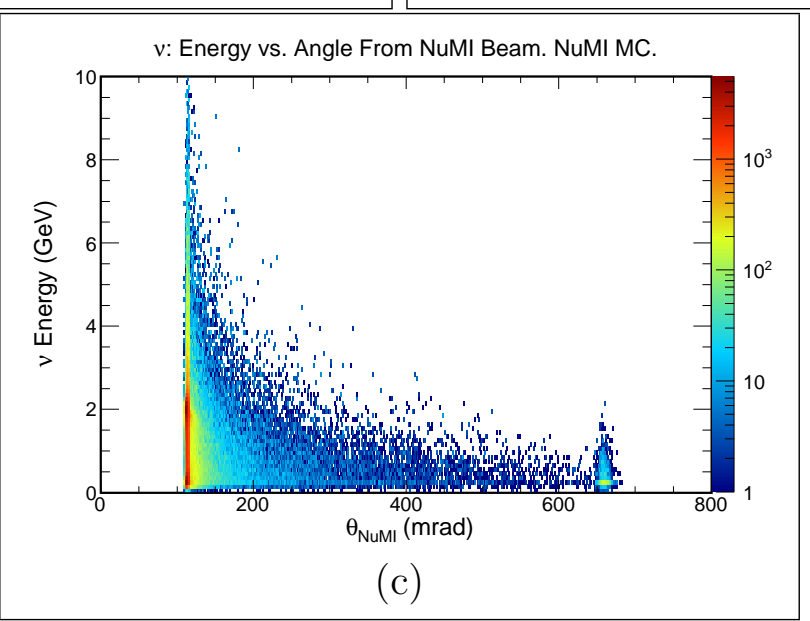

Figure 4.4 Meson Momentum And Neutrino Energy As A Function Of The NuMI Angle. (a) Charged pions and kaons longitudinal momentum as a function of $\theta_{\mathrm{NuMI}}$, (b) charged pions and kaons transverse momentum as a function of $\theta_{\mathrm{NuMI}}$, and (c) neutrino energy as a function of $\theta_{\mathrm{NuMI}}$. MC simulation.

The initial purpose of this detector was to serve as a prototype, and then be moved underground to become the near detector. For this reason it is known as the Near Detector On the Surface (NDOS). However, the collaboration decided to build a totally new and 
improved near detector. As a result the NDOS now serves as a testing facility for various hardware and software purposes.

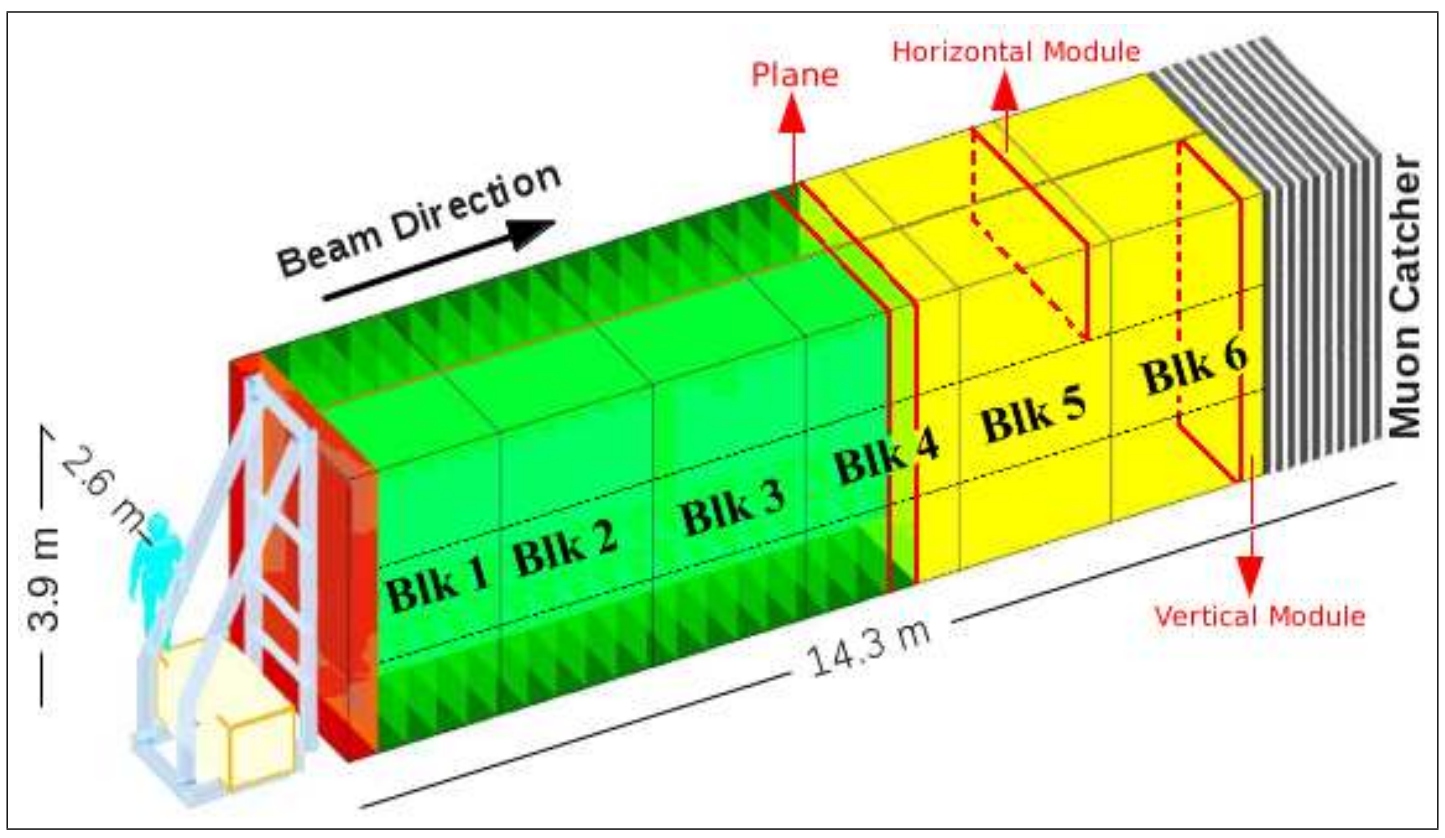

Figure 4.5 Drawing Of The Prototype Detector. The Prototype detector is a collection of six blocks, each made of 31 planes. The neutrino beam goes, in the direction indicated by the black arrow, upstream to downstream. At the end of the six blocks there is a muon catcher that helps to reconstruct the energy of the most energetic muons. Artistic representation.

The NDOS is $3.9 \mathrm{~m}$ high $(Y), 2.6 \mathrm{~m}$ wide $(X)$, and $14.3 \mathrm{~m}$ long $(Z)$, as shown in figure 4.5 . It is a modular structure made from highly reflective PVC extrusions [83]. Each extrusion has 16 cells, and each cell has a $3.9 \times 6.0 \mathrm{~cm}^{2}$ transverse area (see figure 4.6 for an illustration of a single cell). Inside each cell there is a looped wavelength shifting (WLS) fiber, both ends of the fiber are placed against the face of an avalanche photodiode (APD), and the fiber covers the full cell length laid in a U-shape. Extrusions are arranged in modules, there are two extrusions per module. Modules are arranged in planes, there are two $(X)$ or three $(Y)$ modules per plane. A block is an array of interspersed vertical (16) and horizontal (15) planes. This arrangement of interspersed planes gives the $X-Y$ coordinate system of the 


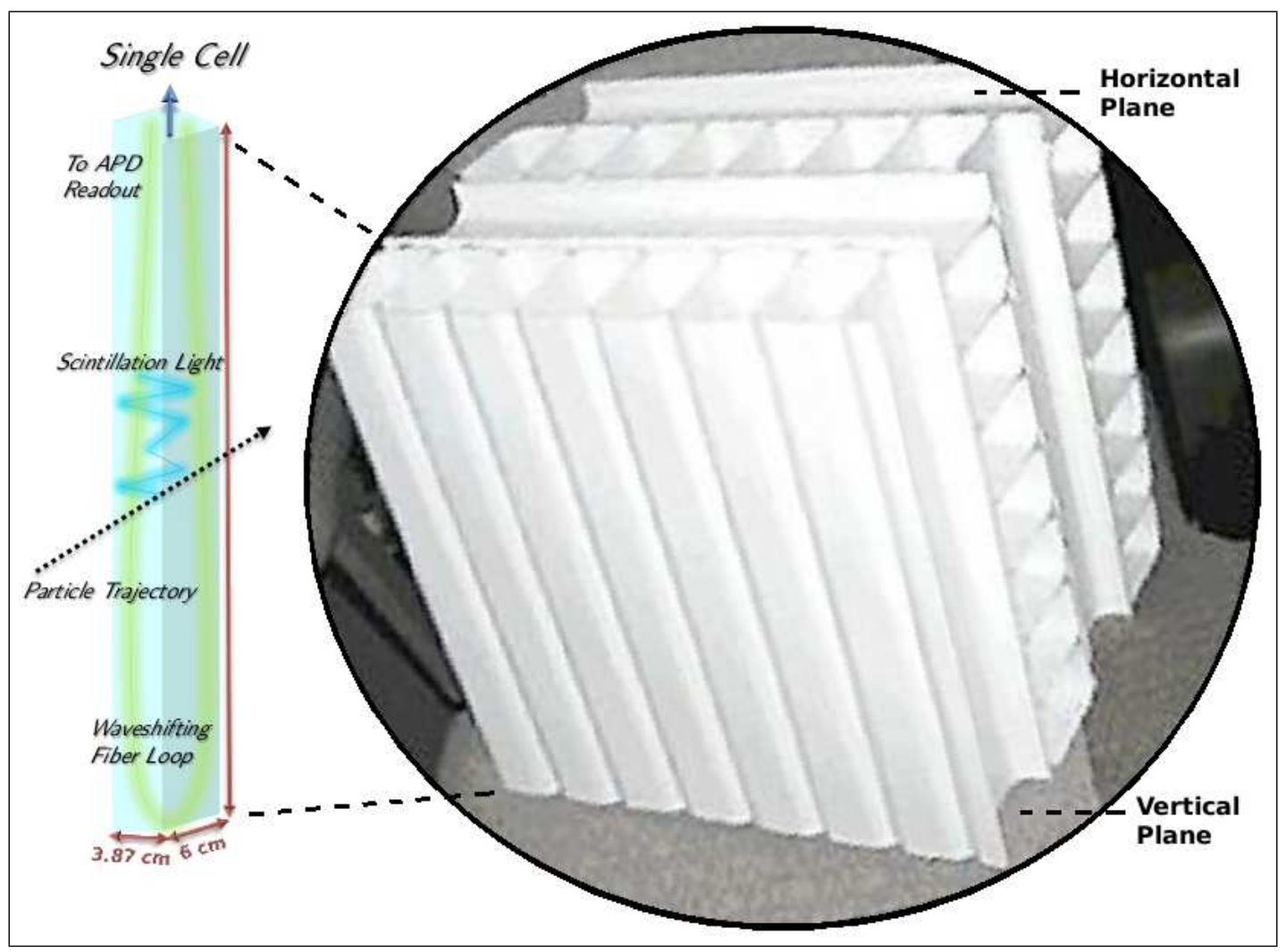

Figure 4.6 NO $\nu$ A Extrusions And Cells. Sample NO $\nu$ A PVC extrusions arranged in a modular collection of vertical and horizontal planes. Artistic representation of a single cell [82].

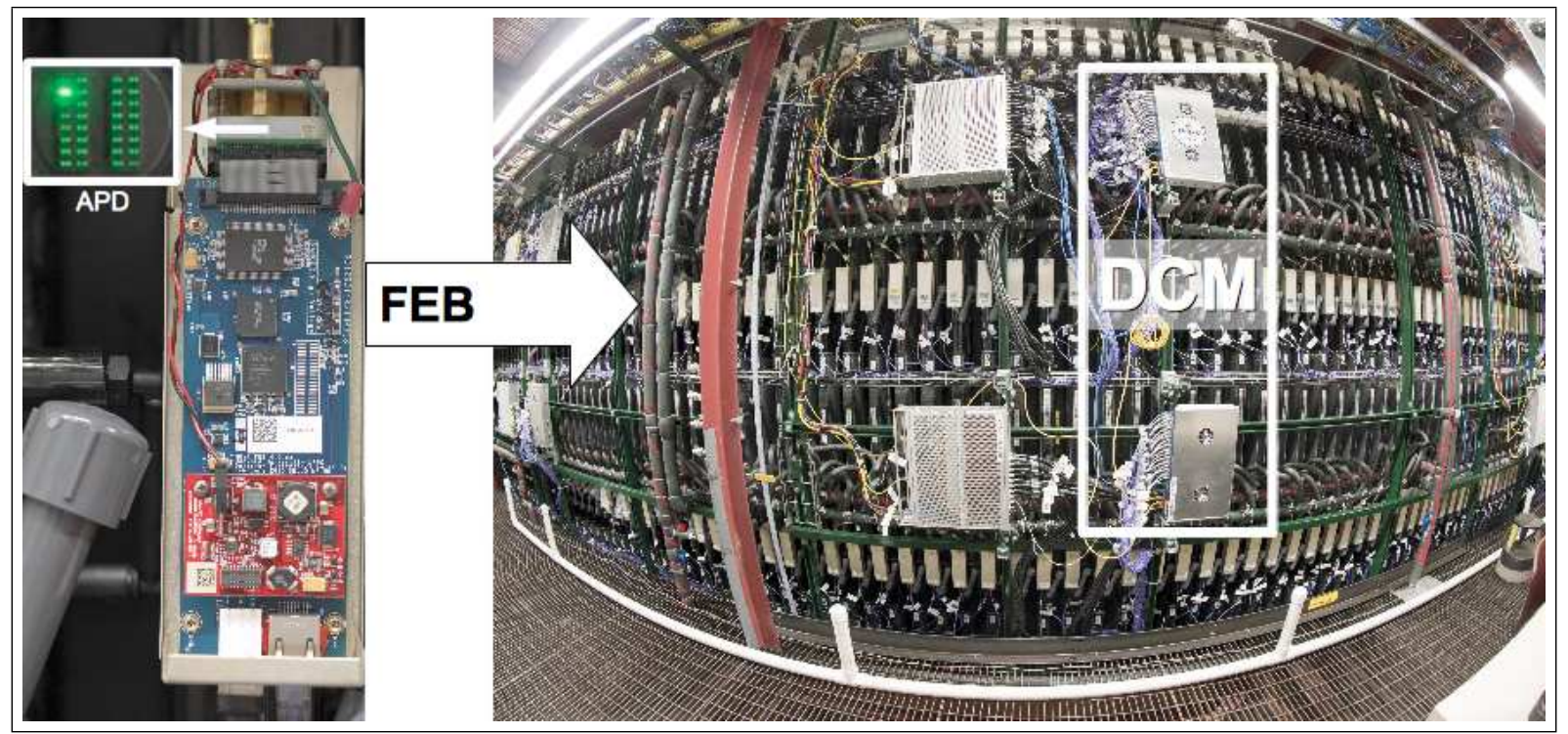

Figure 4.7 Side View Of The Prototype Detector. Horizontal planes readouts. Avalanche photodiode, front-end board and data concentrator modules [84]. 
detector. The NDOS bulk is 6 blocks long. Downstream of the detector there is a muon catcher, which is a collection of interspersed extrusions and ten $10 \mathrm{~cm}$ thick steel plates. At the end of the muon catcher, four more planes are positioned in the usual vertical-horizontal arrangement used for containment purposes, as seen in figure 4.5.

Each APD contains 32 channels or pixels, where each pixel is exposed to both ends of a single fiber in a cell. Each APD is mounted on a front-end board (FEB) which reads and digitizes the signal ${ }^{3}$. The digital output of each FEB is sent to a data concentrator module (DCM) to consolidate the information. Each DCM reads 64 FEB (figure 4.7 illustrates the arrangement of the various hardware components). All the electronics described above are mounted on the west side of the detector, as seen in figure 4.7, for the horizontal planes, and on top of the detector for the vertical planes.

The segmented nature of the $\mathrm{NO} \nu \mathrm{A}$ detectors allows for multiple sampling of the energy depositions of muons and electromagnetic showers ${ }^{4}$. The radiation length of the detectors $\left(X_{0}\right)$ is $X_{0} \approx 40 \mathrm{~cm}$, and the Molière radius is $R_{M} \approx 10 \mathrm{~cm}$. The $X_{0}$ of the detectors allows to get a very detailed sample of $2 \mathrm{GeV}$ electron showers which are, on average, about $9 X_{0}$ in length [85]. Photon showers are, on average, about $9 X_{0}$ long as well, however the gap these leave between the vertex and the start of the shower, which is $X_{0}$ long, provides an element of discrimination. With about 7 cells per $X_{0}$, the energy depositions are extremely useful in particle identification. This same powerful feature is used in muon identification. The signature of a $\nu_{\mu}$ event in the detectors is a muon, and the experimental setup of $\mathrm{NO} \nu \mathrm{A}$ allows to identify and study them with great precision and detail. Identifying electrons is extremely important for the $\nu_{e}$ appearance measurement. A background to electrons are the

\footnotetext{
${ }^{3}$ See section 4.2 .4 .

${ }^{4}$ The segmented geometry of the $\mathrm{NO} \nu \mathrm{A}$ detectors is optimized for the identification and measurement of $\nu_{e} \mathrm{CC}$ interactions, as discussed at the end of section 4.2.
} 
photons that result from $\pi^{0}$ decays.

\subsubsection{Liquid Scintillator}

The primary target in the detectors is the carbon nucleus. About $80 \%$ of the carbon nuclei in the detectors come from the liquid scintillator (LS) blend that fills the PVC extrusions. This blend is $95.79 \%$ mineral oil, used as a solvent, $4.11 \%$ scintillating pseudocumene, and $0.10 \%$ waveshifters [86]. When ionizing particles traverse the LS, ultraviolet light is emitted [87]; the emitted wavelengths are then shifted by the waveshifters to the violet-blue range. This light bounces, on average, 10 times inside a cell before it is captured by the WLS fiber. In order to avoid light losses between emission and absorption, the PVC walls have $12 \%$ $\mathrm{TiO}_{2}$ which gives a reflectance of about $90 \%$ for green wavelengths [21].

\subsubsection{Wavelength Shifting Fiber}

Wavelength shifting fibers are a critical component in the $\mathrm{NO} \nu \mathrm{A}$ detectors. These collect light emitted from the LS and transmit it into the APDs. Inside the far detector's cells the light travels as much as $16 \mathrm{~m}$, which requires shifting of the wavelengths from the short attenuation lengths of the blue (about $4 \mathrm{~m}$ ) to the longer attenuation lengths of the green (about $15 \mathrm{~m}$ ). In addition, the APDs are more efficient in the green than in the blue light. The spectra of the light emitted by the fiber as a function of the illumination point is presented in figure 4.8. The highest light power is the spectrum for the light shined into the fiber at $1 \mathrm{~m}$ from the readout. The lowest light power is the spectrum for the light shined into the fiber at $26 \mathrm{~m}$ from the readout. Intermediate spectra are for illumination points at $1 \mathrm{~m}$ intervals. The various spectra show how the peak of the distribution rapidly transforms 


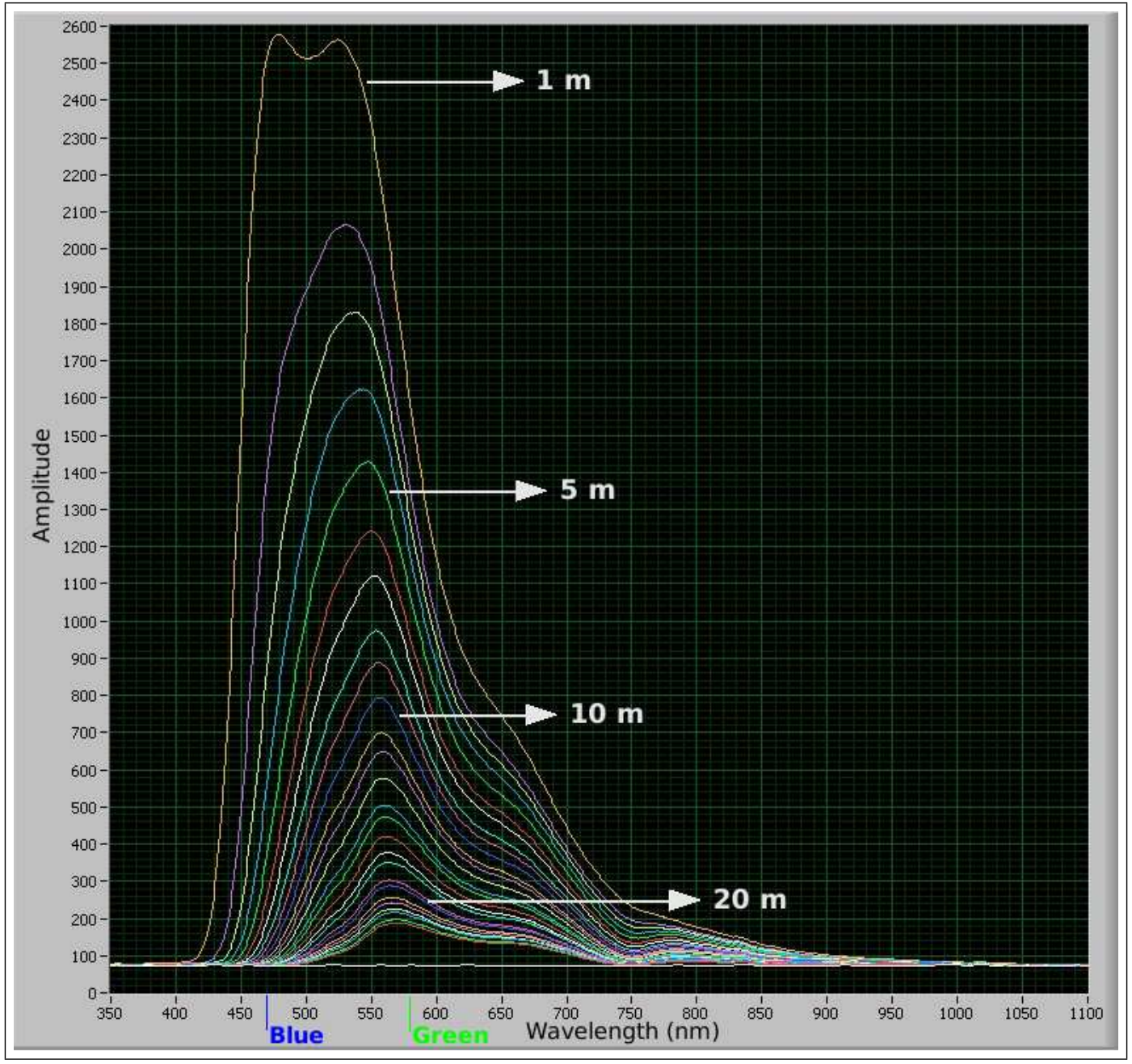

Figure 4.8 Emission Spectra Of The Wavelength Shifting Fiber. Emission spectra for the WLS fiber at various illumination points [88]. 


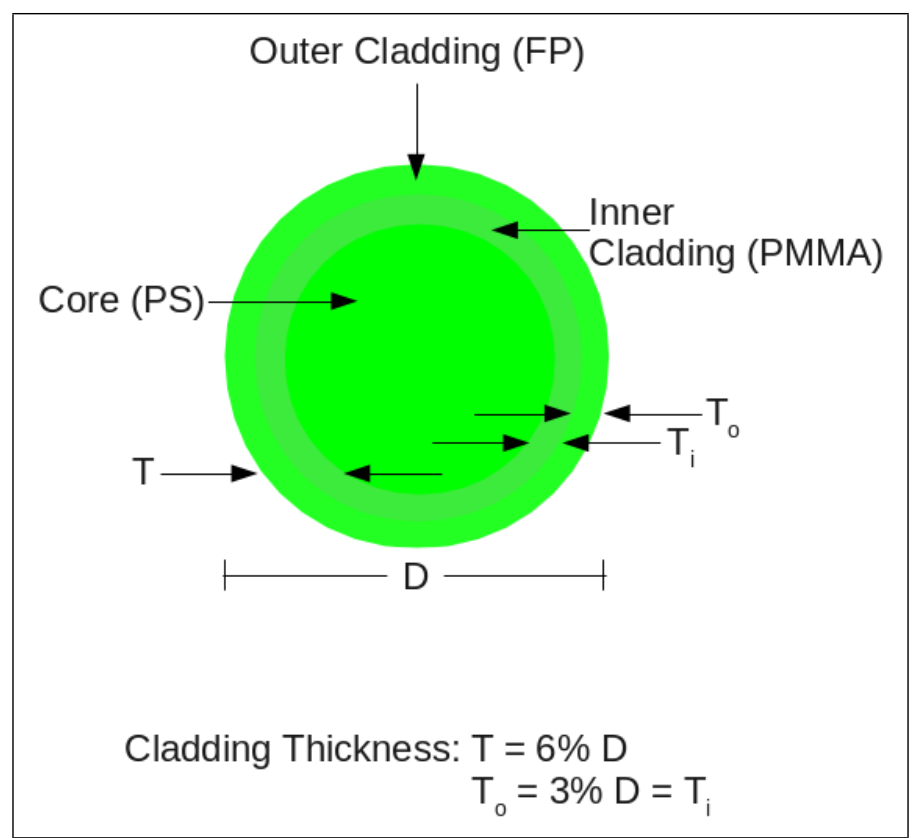

Figure 4.9 Wavelength Shifting Fiber Transverse Area.

from the $470 \mathrm{~nm}$ of the blue wavelength to the $580 \mathrm{~nm}$ of the green as the illumination point moves father apart from the readout.

The WLS fiber used by $\mathrm{NO} \nu \mathrm{A}$ is $0.7 \mathrm{~mm}$ in diameter (D). This features a polystyrene (PS) core with a refractive index of 1.59, surrounded by a polymethylmethacrylate (PMMA) inner cladding with a refractive index of 1.49 , and a fluorinared polymer (FP) outer cladding with refractive index of 1.42 , as shown in figure 4.9. This multicladding structure produces $50 \%$ higher yield than a single cladding fiber due to a large trapping efficiency [89].

\subsubsection{Avalanche Photodiodes}

The NO $\nu$ A collaboration selected APDs as photodetectors due to their high quantum efficiency and low cost. The $85 \%$ quantum efficiency enables the use of the long cells in the far detector ${ }^{5}$. The average signal for muons traversing a cell perpendicular to its walls is

\footnotetext{
${ }^{5}$ Photomultiplier Tubes have a $10 \%$ quantum efficiency under similar circumstances.
} 
30 photons at the face of the APD, which gives about 25 photoelectrons. This signal must be distinguishable from high frequency noise. APDs are made from silicon, which features a thermal generation of electron-hole pairs that mimic the signal from the fibers. These electrons are amplified at the diode junction and appear as the input to the pre-amplifier, thus contributing directly to the noise. In order to achieve a signal to noise ratio of 10:1, the APDs are cooled to $-15{ }^{\circ} \mathrm{C}$.

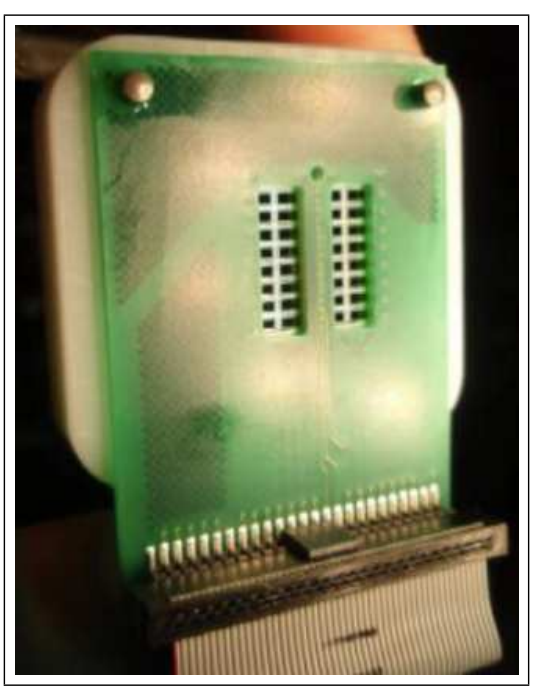

Figure 4.10 Avalanche Photodiode.

The light output of the fiber is absorbed in the APD collection region, shown in figure 4.10, where electron-hole pairs are generated and, under the influence of an applied electric field, the electrons propagate to the p-n junction. At the junction, the electric field, which determines the multiplication of the current, is high enough to produce avalanche multiplication of electrons. The current generated from these electrons is the output signal of the APD [21]. 


\subsubsection{Front-End Boards}

The readout of the $\mathrm{APD}$, at the $\mathrm{FEB}$, requires a pre-amplifier that can sample the signal throughout a $10 \mu$ s time window. The signal from the APD is amplified and shaped by a high-gain integrating amplifier with a shaping time of about $350 \mathrm{~ns}$, and the output is stored in a switched capacitor array (SCA) every 500 ns. The SCA contains 64 samples taken 500 ns apart for the 32 channels in the APD. The waveform is designed to have a 380 ns rise time and 7000 ns fall time [90]:

$$
F(t) \propto\left(1-e^{-t / 380 \mathrm{~ns}}\right) e^{-t / 7000 \mathrm{~ns}}
$$

These choices of rise and fall times are intended to minimize the overall noise induced by the electronics. Zero suppression is performed at $15-20$ photoelectrons $^{6}$ via a dual correlated sampling (DCS) algorithm which subtracts the signal from the baseline. The DCS establishes a rising edge triggered threshold under which the sampling points are zero suppressed. The DCS algorithm then subtracts the sample taken right before the threshold is crossed from the sample, with maximum amplitude, taken right after the threshold is crossed. This threshold is set independently for each channel of the detector. Fits to the shape of the waveform recover the timing and pulse height information [91].

The best timing resolution with this system is $500 / \sqrt{12} \mathrm{~ns} \approx 145 \mathrm{~ns}$. The digitized differences are stored in a field programmable gate array (FPGA) for transmission to the data acquisition (DAQ) system. Digital signal processing algorithms could be encoded in the FPGA firmware to improve both the signal-to-noise ratio and timing resolution. A FPGA

\footnotetext{
${ }^{6} \mathrm{ADC}$ and photoelectrons are proportional. See section 4.2.6 for a discussion on energy calibration.
} 
produces timing markers at periodic intervals of $50 \mu$ s interspersed with digitized hits [92].

\subsubsection{Data Acquisition System}

A DCM is a custom electronic component used to consolidate and concentrate the data from 64 FEBs. A DCM programs, configures, and monitors each FEB, and passes the timing system clock (TSC) and synchronization command to each FEB. The DCM-FEB communication is through point-to-point serial data links with dedicated differential pair lines for clock, synchronization, command and data. The digitized hits are consolidated by the DCM FPGA to $50 \mu$ s time slices containing data from all 64 FEBs. An application further consolidates this data to a longer $5 \mathrm{~ms}$ time slice and routes this time slice to a downstream buffer node for processing. All DCMs throughout the detector send hit data corresponding to one $5 \mathrm{~ms}$ time slice to a buffer node [92].

A buffer node is a commodity server residing in a 140 buffer nodes buffer farm. An external global trigger system provides triggers to a buffer node in the form of a start time and a time window, so that an application can select data corresponding to this time window from buffered data. Only trigger-selected data are written by the buffer nodes to a downstream data logger.

A data logger is a program that receives trigger-selected data from the buffer farm to format into an event. A trigger issued to the buffer farm is also issued to the data logger application for validation purposes. The event created by the data logger is written to disk, and then archived to Fermilab's mass storage. Events are also written by the data logger to a shared memory segment, from which a dispatcher application can serve the data to a quasi-online external client application.

All FEBs and DCMs throughout the DAQ system are synchronized through the use of 


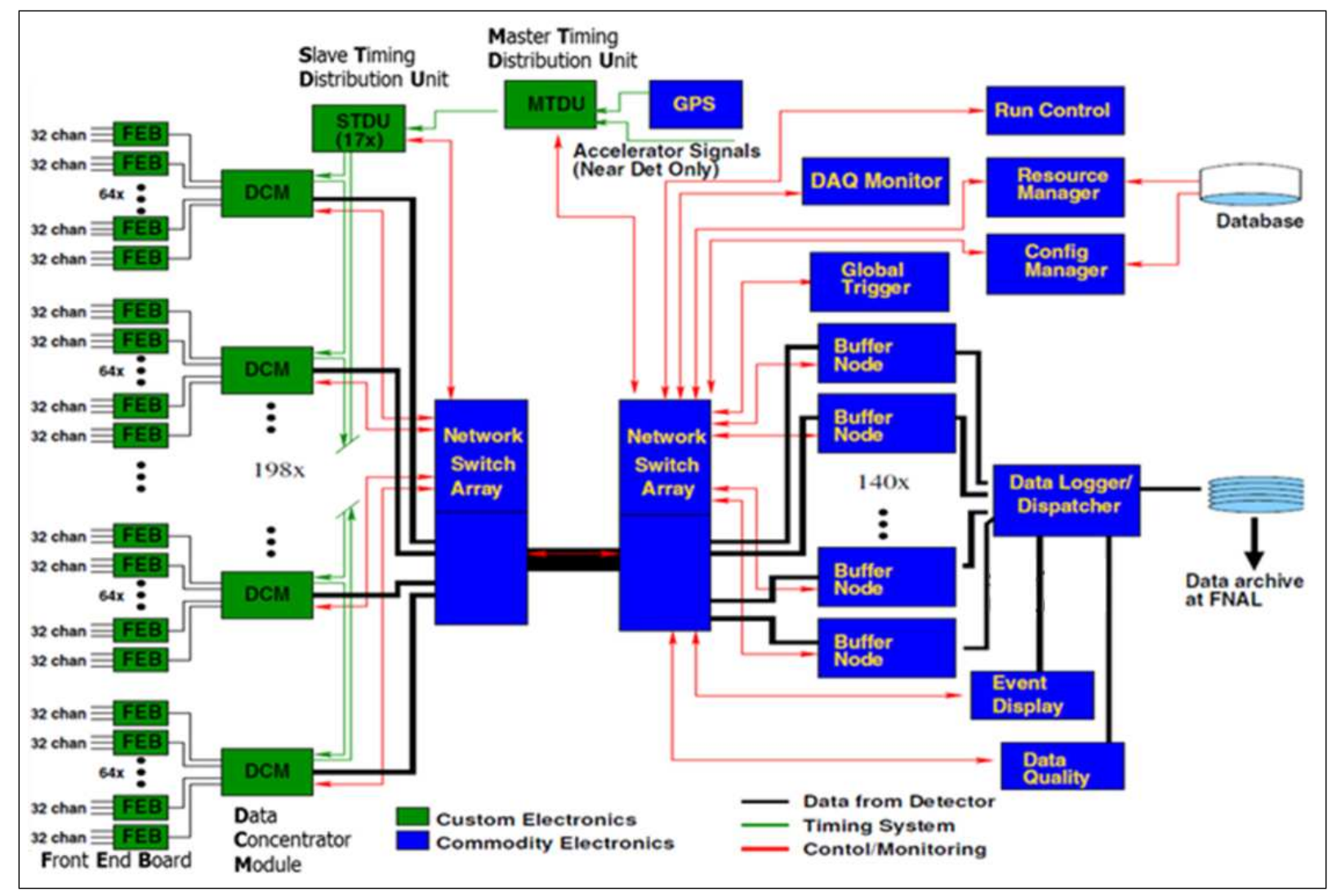

Figure 4.11 Schematic Overview Of The Data Acquisition System [92]. 
a sophisticated timing system. This system provides a stable master clock line that permit the time stamp counters that are present on the FEBs, DCMs, and the timing system to be loaded and synchronized with a universal wall time based off of a link to the GPS. The timing system can achieve unit to unit synchronization that is accurate to within one clock cycle. This system is also used to time stamp the beam spill information coming from the Fermilab accelerator complex [91].

Each timing distribution unit (TDU) has four differential pair lines for communication: synchronization, command, clock, and synchronization echo. The synchronization echo line is used to compensate for cable length propagation delays. Refer to figure 4.11 for a DAQ system schematic that illustrates the various steps and components involved in the data processing.

Individual data output files are saved by SubRuns, which are either an hour long, or 512 MB in size, which ever happens first. SubRuns are grouped in Runs which can have up to 24 SubRuns. Any time that the DAQ systems are stopped, that is the end of the SubRun and Run in progress. The SubRuns that recorded the NuMI spills have one event per spill, and each event is $500 \mu$ s long [92].

\subsubsection{Performance And Calibration Of The Prototype Detector}

The NDOS started its physics run in October 2010 during which cosmic ray data, and $\nu_{\mu}$ and $\bar{\nu}_{\mu}$ data from the NuMI beam were collected. The collaboration intended to fully instrument the detector with APDs. However, various problems occurred during commissioning, and the majority of the APDs were damaged due to moisture on their face coming from condensed water deposited when the detector was cooled down. About a third of the APD set survived the problems. The APDs that survived were strategically located throughout the detector in 


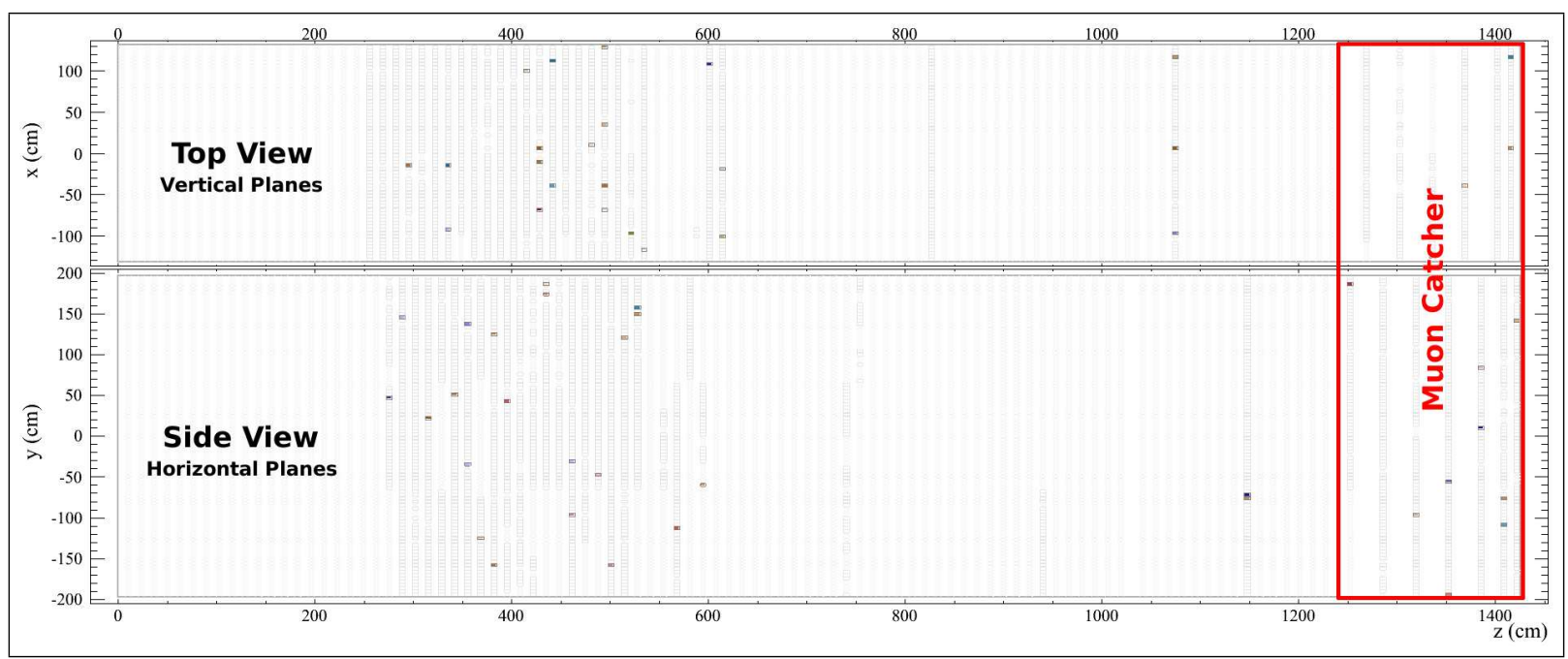

Figure 4.12 Event Display Of The Prototype Detector. Vertical planes make the top view, and provide the horizontal $(X)$ coordinate. Horizontal planes make the side view, and provide the vertical $(Y)$ coordinate.

order to optimize the observation and measurement of neutrino interactions in the detector. Most of the available APDs were gathered together in a region in order to identify the neutrino interaction point, and the rest of the available APDs were located in strategic individual planes in order to record the full length of the muon tracks. The plan with the muon catcher was to have it fully instrumented. However there are some channels maps with some regions that were also partially instrumented.

An event display of the detector is presented in figure 4.12. Cells with active APDs are drawn with black lines, while inactive cells are drawn in light grey. The top view is made from vertical planes which provide the $X$ coordinate; the side view is made from horizontal planes which provide the $Y$ coordinate. Neutrinos from the NuMI beam enter the detector from the bottom $(Y=-200 \mathrm{~cm})$. The bulk region, where most of the APDs are located, is centered at $Z=400 \mathrm{~cm}$. If APDs became available, these were installed to improve muon identification, and if APDs failed, these were removed. This configuration is known as NDOS run II, and the data recorded under this configuration goes from October 2011 until April 
2012, during which there were $1.67 \times 10^{20}$ protons on target $(\mathrm{POT})$. The APDs operated warm to avoid further losses. The active channels map for each SubRun is known, and in general, such maps change frequently.

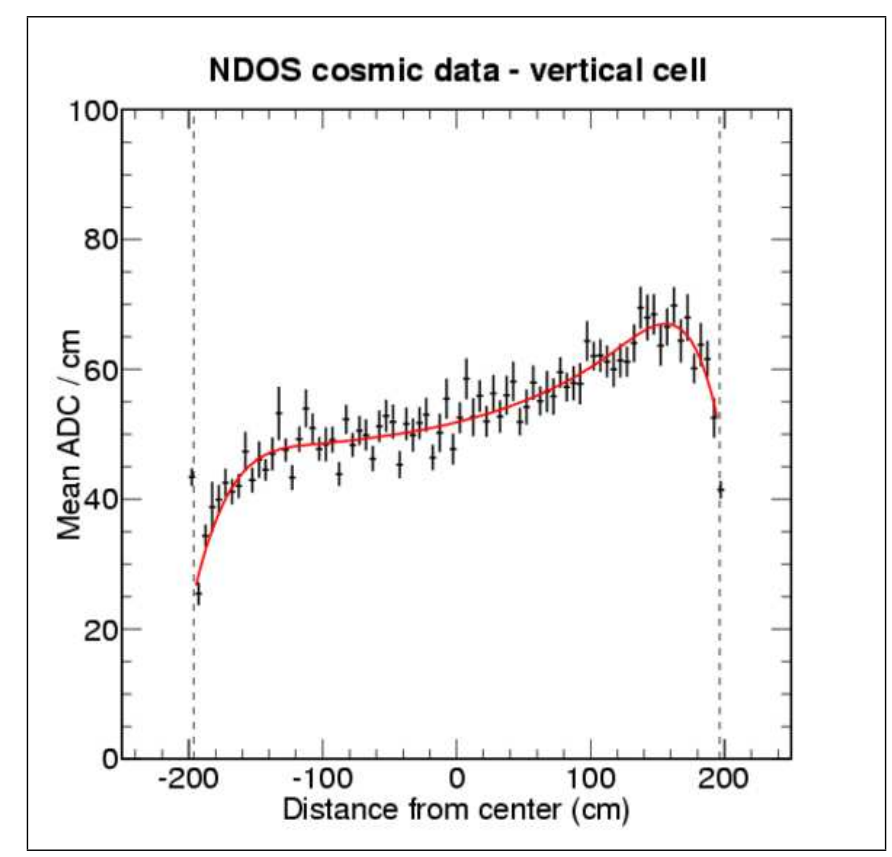

Figure 4.13 Cosmic Data Of The Prototype Detector. Distance from the center of the detector vs. mean ADC value per cm. Attenuation corrections come from the fit. The readouts are in the right. Official $\mathrm{NO} \nu \mathrm{A}$ NDOS figure [81].

The performance and calibration of the detector were initially studied using cosmic ray data. The energy calibration procedure used energy deposition of cosmic rays at different locations in the detector. The light level is measured, initially, in analog to digital converter (ADC) units of collected charge. Apart from a drop in the average light at short distances from the APD (near the beginning of the PVC cell), the mean observed light level decreases with increasing distance of the hit from the APD, as seen in figure 4.13. Here, the position $(W)$ of a hit is measured from the center of the detector. The electronics are beyond the $W=200 \mathrm{~cm}$ point. The goal of the energy calibration is to make hits depositing the same energy, but located at different distances from the readouts, result in the same corrected 
energy measurement. The mean $\mathrm{ADC} / \mathrm{cm}$ is a representation of the light attenuation per cell. The rapid fall at $Y<-140 \mathrm{~cm}$ is due to the sum of two exponential functions that represent the light attenuation in the fiber [93]:

$$
A(r) \sim A_{S} e^{-r / R_{S}}+A_{L} e^{-r / R_{L}} .
$$

Here $r$ is the distance traveled by the light inside the fiber, $R_{S}=289.5 \mathrm{~cm}$ is a short attenuation length, and $R_{L}=852.3 \mathrm{~cm}$ is a long attenuation length. Light entering the fiber is split up according to the constants $A_{S}=0.3137$ and $A_{L}=0.1669$, and propagated to the readouts according to the two attenuation lengths [93]. The rapid fall at $Y>140 \mathrm{~cm}$ is due to light absorption at the manifold that guides the fibers to the APDs.
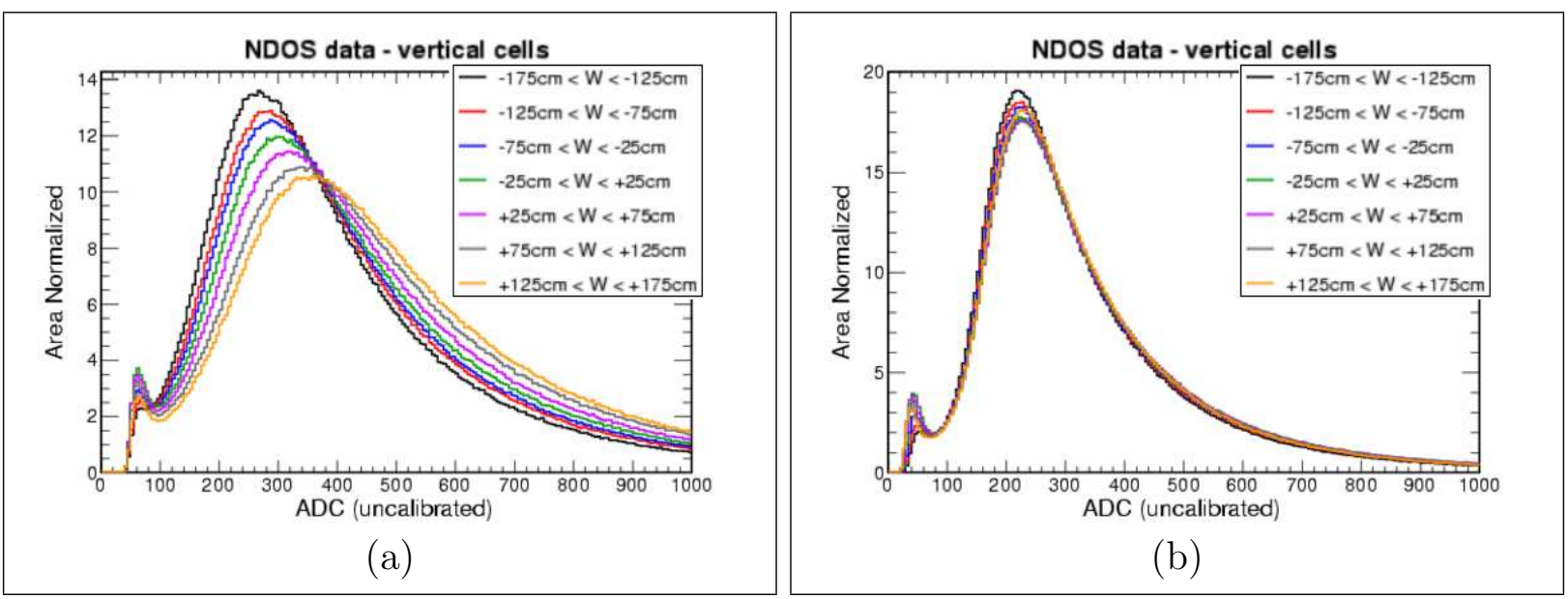

Figure 4.14 Energy Calibration Of The Prototype Detector. ADC distributions for various $W$ slices (a) before and (b) after attenuation corrections. Official $\mathrm{NO} \nu \mathrm{A}$ NDOS figure [81].

Muons passing through the detector exhibit an uncorrected light level distribution with a mean that shifts from about $400 \mathrm{ADC}$, at the closest distance to the readouts, to about $300 \mathrm{ADC}$ at the farthest distance to the readouts, as shown in figure 4.14a. The small peak at low light levels is the tail of the APDs noise distribution above threshold, and is due to 
cells in which the muon passes entirely through the detector PVC. After the attenuation corrections are applied the light level distributions overlap, as seen in figure 4.14b, so that the energy measurement is then independent of $W$.

To approximate to photoelectron (PE) units, a simple rescaling of ADC is carried out: $A D C / P E=2.275$ [93]. The linear relation between $\mathrm{ADC}$ and $\mathrm{PE}$ is seen in figures 4.15a and 4.15b, where the same conversion factor appears for the MC simulation and the data, respectively. Application of the position dependent corrections discussed above yielded a corrected number of photoelectrons (PECorr). The corrections are a function of $W$, which means that ADC and PECorr are not proportional, as seen in figures $4.15 \mathrm{c}$ and $4.15 \mathrm{~d}$. The initial assumption is that at $W=0: \mathrm{PE}=1=$ PECorr [93]. The poor $\chi^{2}$ tests indicate that the two quantities do not follow a straight line correlation.

To convert from PECorr to an absolute energy deposition, the range-energy relationship provided by the Bethe Bloch formula [87] is used. A sample of cosmic ray tracks that clearly stopped within the detector is selected and the reconstructed stopping point determined for each track. With the distance $\left(d_{c}\right)$ from the center of each cell in the track to its endpoint, a relation between PECorr $/ \mathrm{cm}$ and $d_{c}$ is established. The Bethe Bloch curves are integrated to provide the expected $d E / d X$ for every $d_{c}$. The integration takes into account the density of the material that receives the deposited energy, i.e. to distinguish between PVC, scintillator, etc.. The best fit to the profile of the PECorr/cm plot yields a calibration of: $\mathrm{GeV} / \mathrm{PECorr}=4.7807 \times 10^{-5}[93]$, about $50 \mathrm{keV}$ per corrected photoelectron .

The performance of NDOS over time is represented by the light level in a set of test cells. The set of cells is located at the center of the detector in $X-Y$ and at the south edge of the bulk of APDs, $Z=280 \mathrm{~cm}$. During the running of the NDOS there were times with the APDs system cold or warm, and FEBs gains were varied; however, the performance of the 


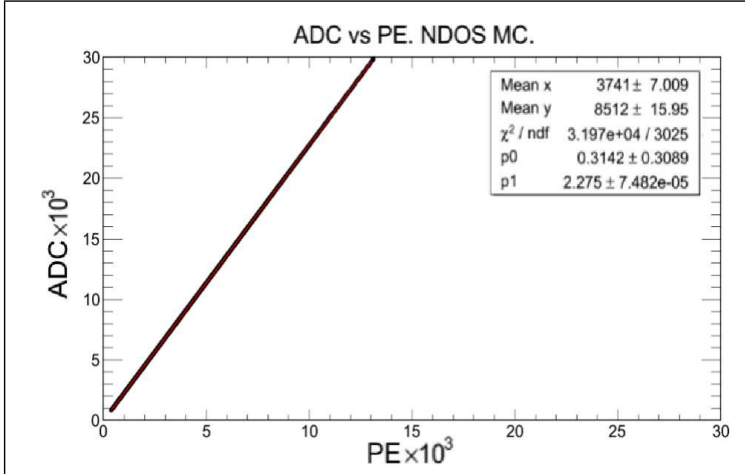

(a)

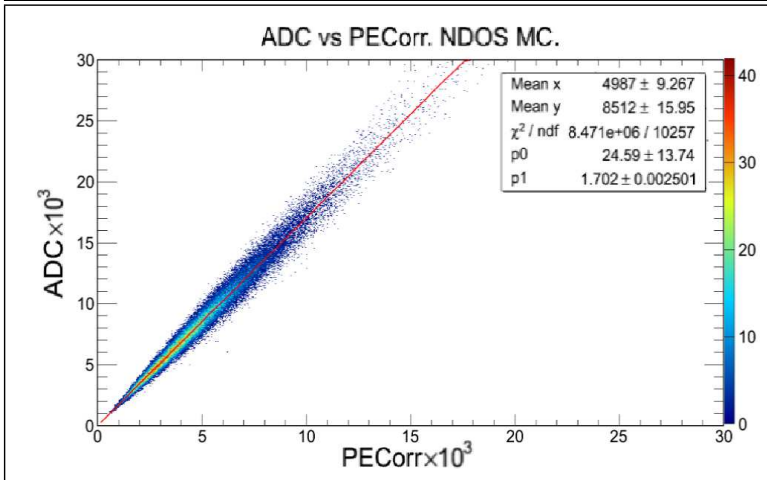

(c)

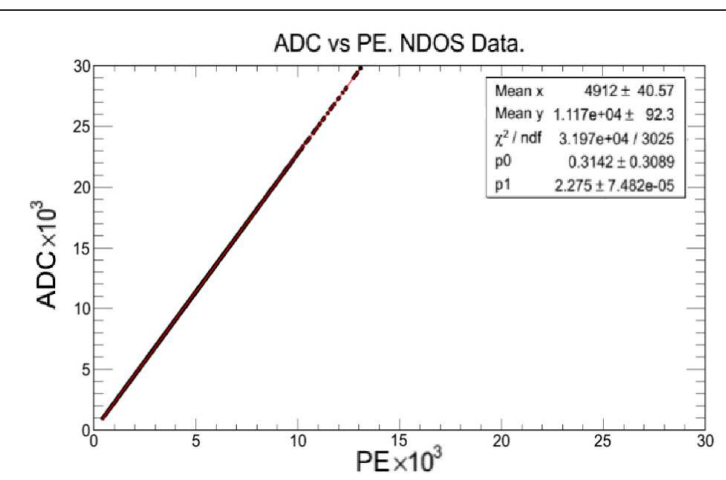

(b)

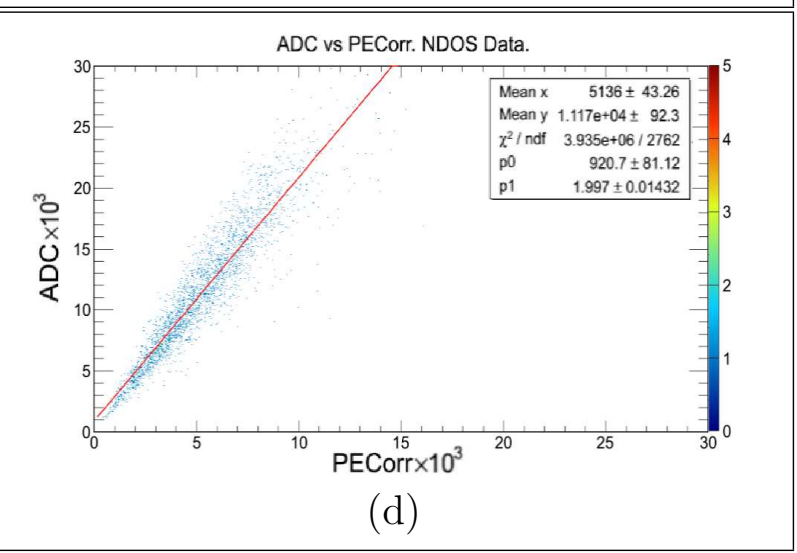

Figure 4.15 Energy Conversion Factors Of The Prototype Detector. ADC vs. PE for (a) MC simulation and (b) data. ADC vs. PECorr for (c) MC simulation and (d) data [94]. 


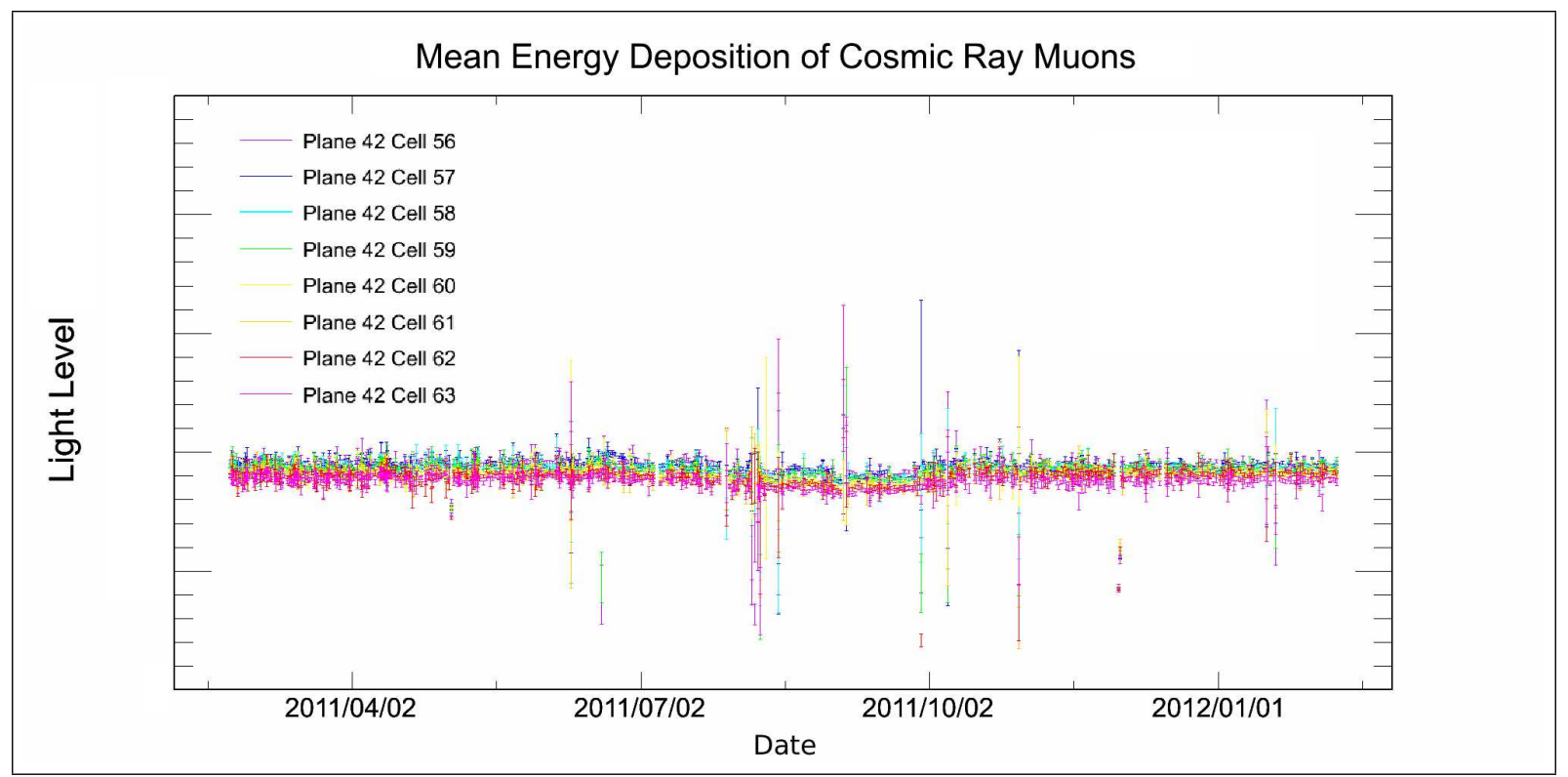

Figure 4.16 Light Level Over Time On The Prototype Detector. Light level on 8 test channels of an APD as a function of time [95].

detector remained stable, as seen in figure 4.16. The time elapsed starts six months prior to the NDOS run II, and ends a month before the end of this run. During the period shown, there were times of cold and warm APD, and the gains varied in the FEB; however, the performance of the detector remained stable.

\subsection{NO $\nu \mathrm{A}$ Software}

To evaluate the biases affecting various physics process involved in the analyses, the $\mathrm{NO} \nu \mathrm{A}$ collaboration developed a Monte Carlo (MC) detector simulation, and track reconstruction programs which could be applied to both MC and data objects. 


\subsubsection{Monte Carlo Simulation}

The $\mathrm{NO} \nu \mathrm{A}$ simulation process begins at the NuMI beam level. The MC simulates the hadron production in the NuMI target, which is followed by the propagation of the produced particles through the target material, the magnetic horns and the decay pipe. The FLUKA software [96] simulates interactions of $120 \mathrm{GeV}$ protons in the NuMI target, and the interaction of secondary particles created in the target as well. The simulation creates output files, which contain the kinematic variables of the generated particles. These files are input to the GEANT4 [97] software, which propagates the particles, and their decay products, from the NuMI target, through the magnetic horns, and down the decay pipe, where weak decays yield neutrinos. If the combination of FLUKA and GEANT4 (known as FLUGG [98]) generates a neutrino that reaches the NDOS, the GENIE [99] software simulates interactions in proportion to predetermined cross sections. The interaction is simulated within the target nucleus, and an intranuclear hadron transport yields identified final state particles with full kinematics.

The NO $\nu$ A collaboration has developed a full simulation of the NDOS using GEANT4 to propagate GENIE generated particles through the detector. The software simulates energy deposition, scattering, and decay processes that affect the particles produced during neutrino interactions. The $\mathrm{NO} \nu \mathrm{A}$ collaboration developed further simulation tools that use the energy depositions in the scintillator to generate blue light, reflect it in the PVC cells until absorbed by wavelength shifting fibers. Equation (4.5) parametrizes the attenuation of the green shifted light as it propagates through the fibers to the APD and the readouts. All the values of variables created by the MC simulations that are available for analysis are named: true values. 
To provide samples with the same biases as the real data, the MC SubRun are created with the same active channels maps. Also, the number of POT used to produce the MC sample is stored in the files, as it is done in the data.

\subsubsection{Reconstruction Tools}

The reconstruction tools developed by the $\mathrm{NO} \nu \mathrm{A}$ collaboration are intended to work with simulated and real values of the variables ${ }^{7}$. The basic unit in the reconstruction process is a hit. A hit is an energy deposition left in the detector by a particle. The simulation provides hits for every interaction of a particle within the detector; there could be many simulated hits inside an individual cell. Data also provides hits, which are energy depositions in a detector cell that generate a detected amount of light, above the electronic noise threshold, at a digitized time, and with a position provisionally given as the center of the cell. Any group of real hits within a cell that share the same time tag will be collected into one hit: a cell hit. Cell hits appear both in simulated and real data of the variables. Cell hits have stored values of: energy, time, and position; which have been properly calibrated (see section 4.2.6).

\subsubsection{The Slicer}

In order to reconstruct a neutrino interaction, whether it is simulated or data, the first stage in the reconstruction is to make slices of cell hits that share common features in space and time. The Slicer sorts the cell hits in an event by time, after which subsets of cell hits (slices) that are grouped by similar times are produced. Different slices are separated by at least

\footnotetext{
${ }^{7}$ Real values of the variables are those provided by the DAQ, and come from real measurements, or data.
} 


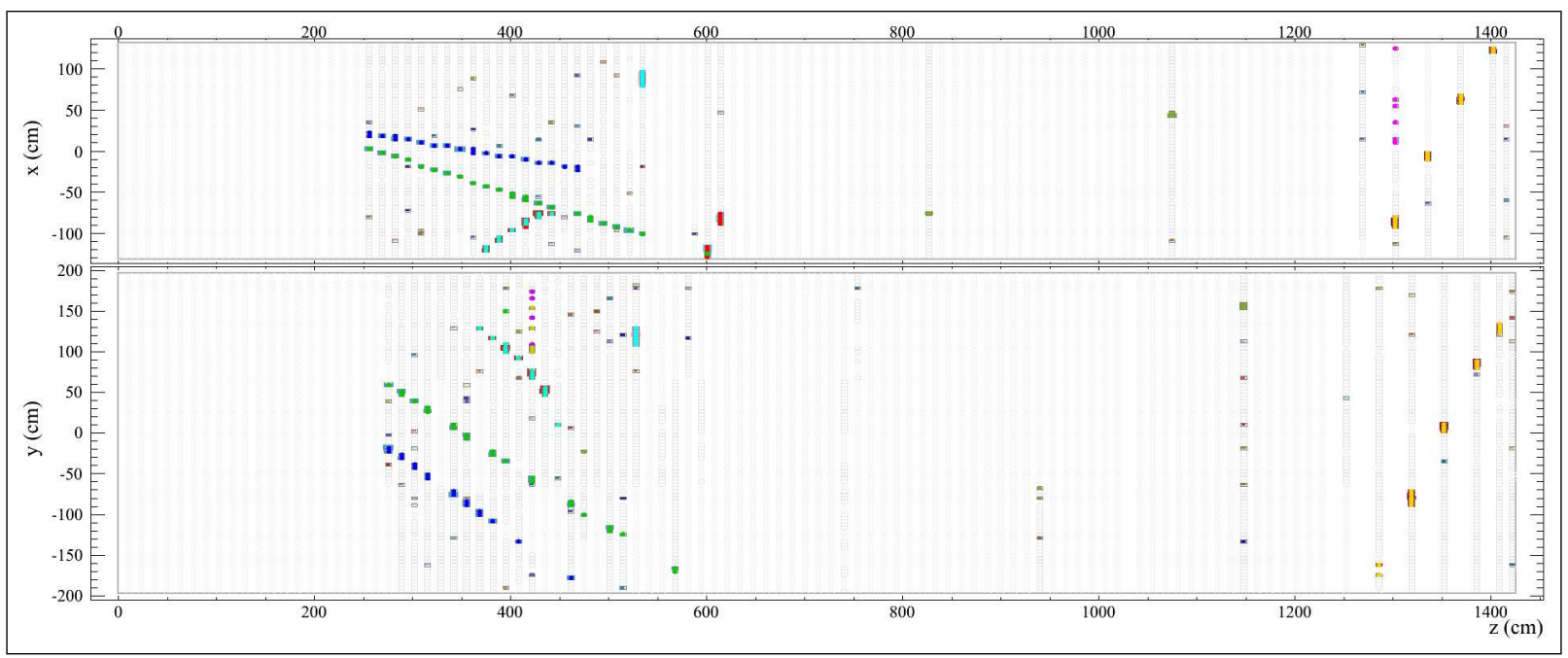

Figure 4.17 Sample Cosmic Ray Event For Slicer Performance. Colors represent the various physics slices.

530 ns. A physics slice is one where the number of cell hits is eight or greater.

Cell hits are considered noise, and removed from a physics slice, if they are isolated from other cell hits in space and/or time. Cell hits with $P E<15$ are also considered noise, and removed from the physics slice: noise subtraction. Cell hits subtracted from a physics slice are not recorded as noise, but are incorporated to the noise slice. After the classification done by the Slicer there would be: physics slices, and a noise slice that collects all the noise hits in the event. In events defined by a $500 \mu$ s time window, the performance of the Slicer is determined by its efficiency $(\epsilon)$ and purity $(p)$ :

$$
\epsilon=\frac{s_{t}}{s_{e}}=96 \%, \quad \mathrm{p}=\frac{s_{t}}{s_{p}}=99 \%
$$

where $s_{e}$ is the total number of true cell hits in the event that are not labeled as noise, $s_{t}$ is the total number of cell hits in the physics slices and not labeled as noise, and $s_{p}$ is the total number of cell hits in the physics slices [100]. Colored dots are drawn on top of the cell hits 
to represent each of the various physics slices, as shown in figure 4.17, for a sample cosmic ray event. The noise slice is not drawn.

\subsubsection{Track Reconstruction}

In $\nu_{\mu} \mathrm{CC}$ interactions, the nominal path of the muon through the detector is a straight line, though multiple scattering can cause noticeable deviations, especially near a stopping point. Nevertheless, tracking muons in the detector is straightforward, and their initial energies can be calculated from the paths lengths, if the muons stop in the detector. The $\mathrm{NO} \nu \mathrm{A}$ collaboration developed a series of tracking algorithms to reconstruct cosmic tracks, tracks from neutrino interactions, and showers. In tests on MC simulated muon tracks in the NDOS, with its large number of inactive channels, the Kalman Tracker algorithm performs better than the alternatives at reconstructing muon tracks produced by neutrino interactions.

To test the performance of the tracker on various kinds of particles, the difference between the true and the reconstructed track lengths $(\Delta L)$ divided by the true track length $(L)$ is examined. The test shows that the tracker is most efficient at reconstructing muon track lengths, and not so efficient at reconstructing the lengths of other particles. This test is most

relevant to this analysis of $\nu_{\mu} \mathrm{CC}$ interactions, where tracking is used to identify a candidate muon track. The track length allows the energy of the muon to be calculated. The remainder of the neutrino interaction energy is determined using the detector as a calorimeter. The overall efficiency $(\epsilon)$ and purity $(\mathrm{p})$ of the tracker are:

$$
\epsilon=\frac{k_{t}}{k_{e}}, \quad \mathrm{p}=\frac{k_{t}}{k_{p}},
$$

where $k_{e}$ is the total number of true cell hits that belong to a particle, $k_{t}$ is the total number 
of cell hits in the reconstructed track that belong to that particle, and $k_{p}$ is the total number of cell hits in the reconstructed track. The efficiency and purity for muons are higher than for protons and charged pions. A summary of the performance of the tracker is presented in table 4.1. For a complete study on the performance of the various NO $\nu \mathrm{A}$ trackers see [101].

\begin{tabular}{|c|c|c|c|c|}
\hline Kalman Tracker & All & $\mu$ & $\mathrm{p}$ & $\pi^{ \pm}$ \\
\hline \hline Efficiency & $75 \%$ & $92 \%$ & $69 \%$ & $68 \%$ \\
\hline Purity & $61 \%$ & $87 \%$ & $37 \%$ & $41 \%$ \\
\hline $100 \% \times \Delta L / L$ & - & $23 \%$ & $131 \%$ & $78 \%$ \\
\hline
\end{tabular}

Table 4.1 Kalman Tracker Performance. Kalman Tracker's efficiency, purity and $\Delta L / L$ for various particles [101].

The Kalman Tracker uses a Kalman filter [102] for track pattern recognition and track fitting. In this application, the filter has the ability to obtain pattern recognition and track fitting in one module, and the capability to find multiple tracks within a group of correlated cell hits. The input of the filter are slices from the Slicer, and the output are 2D and 3D tracks. The $2 \mathrm{D}$ track reconstruction is performed on each physics slice, and separately in each view. Whenever possible, the $2 \mathrm{D}$ tracks found in separate views are matched together to form 3D tracks if these belong to the same physics slice. The Kalman Tracker algorithm is described in appendix B.

The Kalman Tracker does not reconstruct a vertex. Therefore the interaction vertex is placed at the starting (lowest $Z$ coordinate) position of the longest track in the neutrino interaction. The vertex resolution is very good in the three coordinates, about a cell, as seen in figure 4.18. The tails in the distributions (beyond $1 \sigma$ ) account for $15 \%$ of all the tracks. These tails are due to the large number of inactive channels in the detector, but they are sufficiently small to make a reasonable fiducial volume cut based on the coordinates of the vertex. 


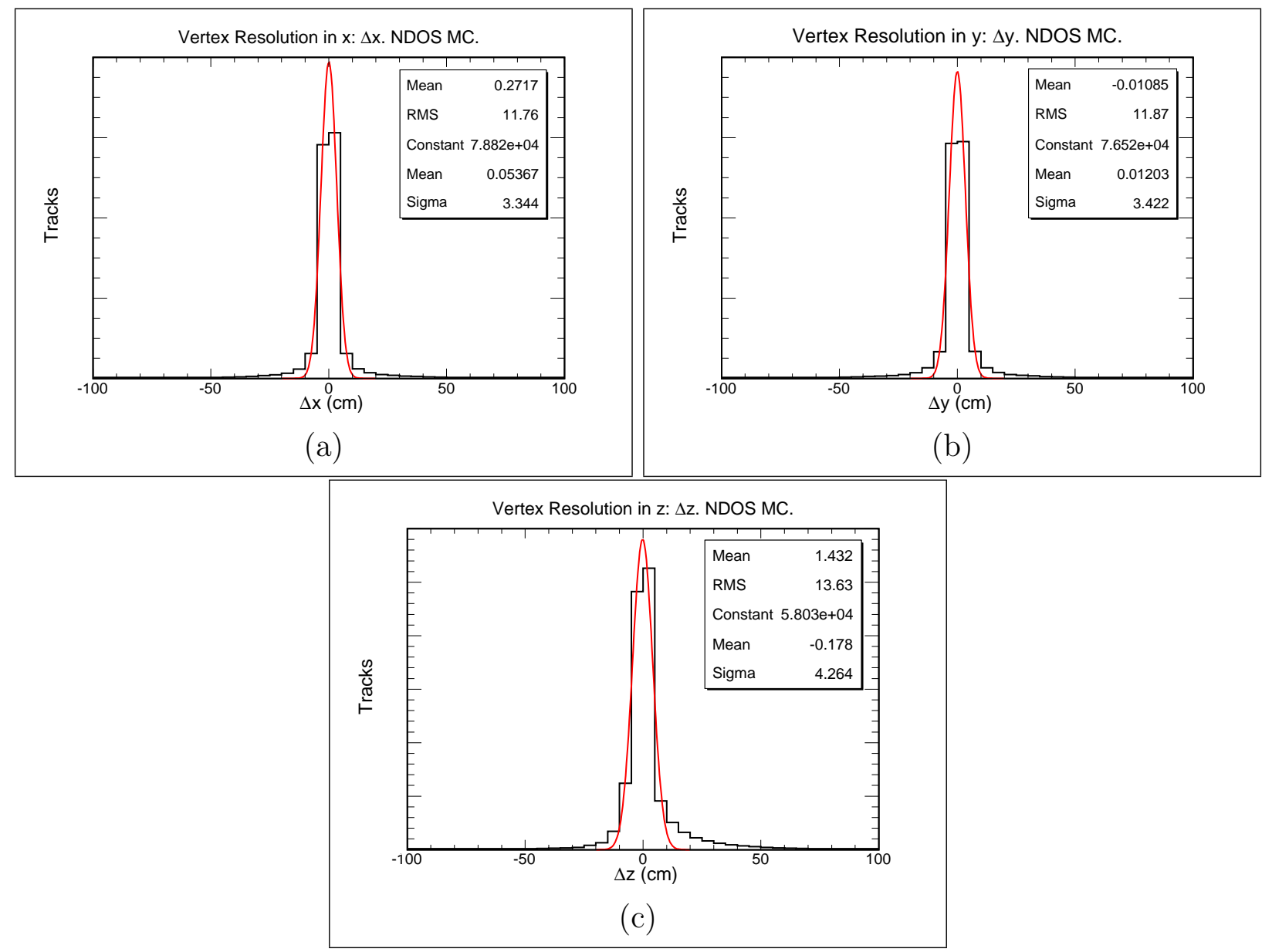

Figure 4.18 Vertex Resolution. (a) $X$, (b) $Y$, and (c) $Z$. MC simulation. 


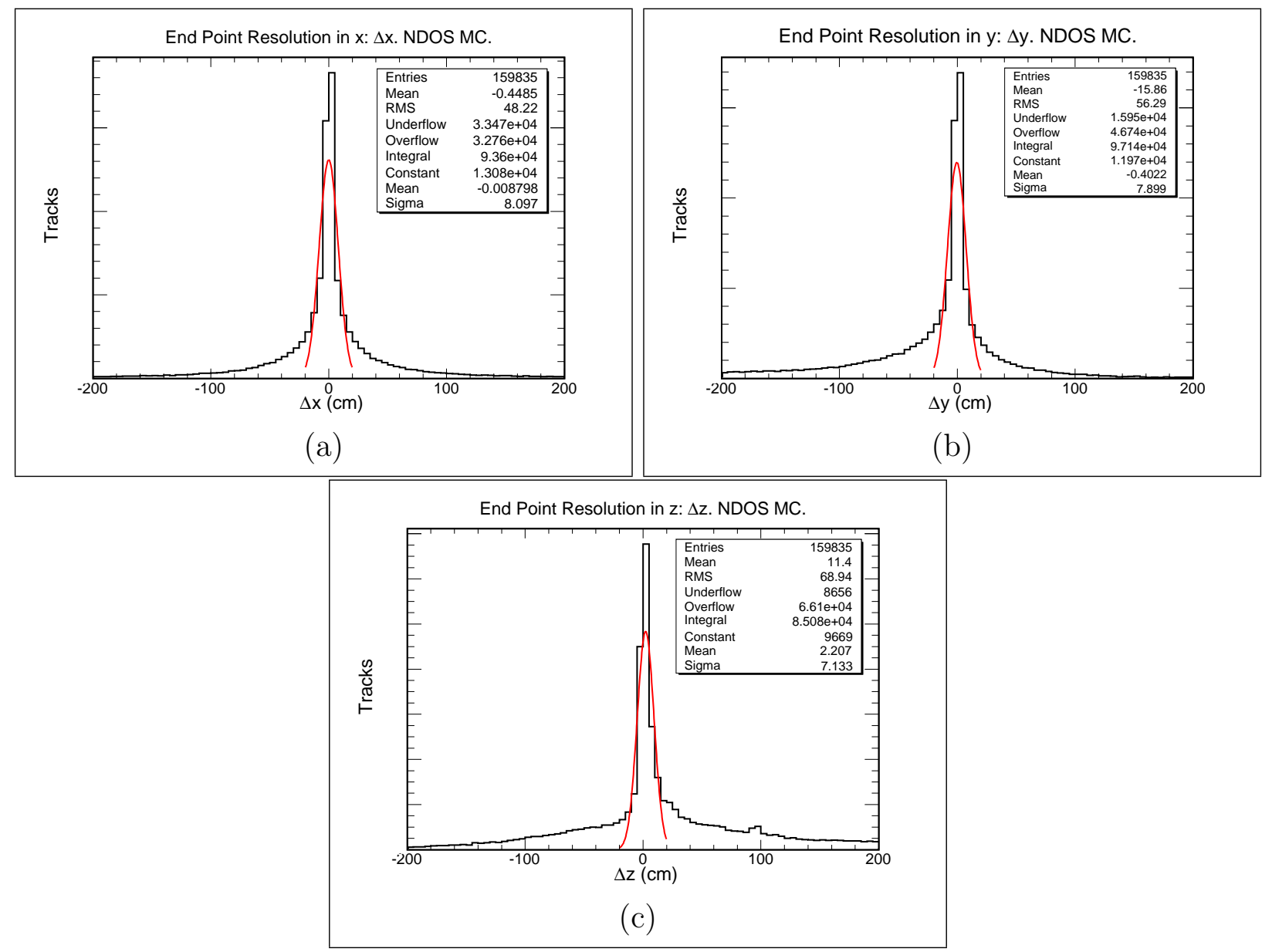

Figure 4.19 Endpoint Resolution. (a) X, (b) $Y$, and (c) Z. MC simulation. 
Finding the endpoint of the longest track has some significant problems. The large gaps in $Z$, with no active channels in the detector downstream of the interaction region, presents a serious obstacle to achieving acceptable performance with the tracker. Therefore, the resolution for the endpoint of the longest track is not as good as the vertex resolution. When muons reach the regions where only a few planes have active channels $(Z>600 \mathrm{~cm})$, the endpoint of long tracks has a large uncertainty, as seen in figure 4.19. The long tails in figures 4.19a, 4.19b, and 4.19c account for: $63.2 \%, 66.4 \%$, and $75.7 \%$ of all tracks, respectively. The overall endpoint resolution in $X$ and $Y$ is about $50 \mathrm{~cm}$, and in $Z$ is about $60 \mathrm{~cm}$. For reference, a $2 \mathrm{GeV}$ muon deposits $120 \mathrm{MeV}$ of energy in $60 \mathrm{~cm}$ of scintillator.

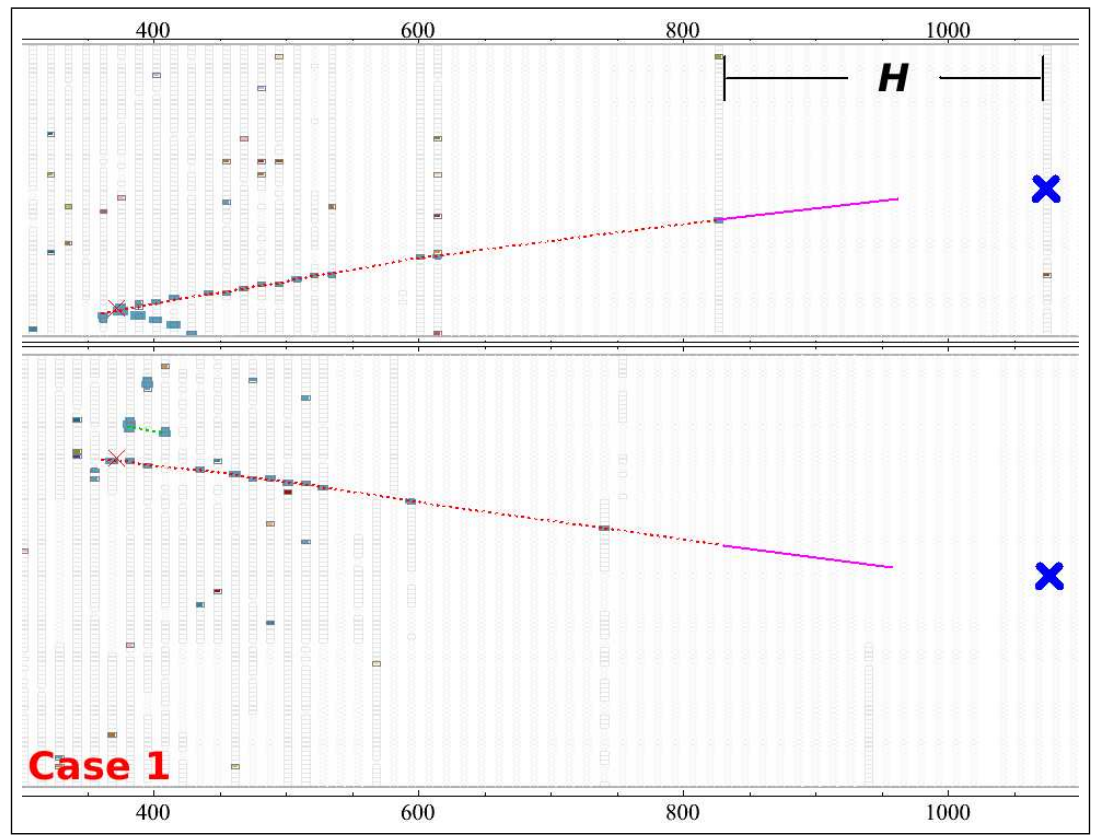

Figure 4.20 Sample Event. Case (1). MC simulation.

Reconstructed tracks have their starting and ending points in the center of an active cell. However, a real muon is not guaranteed to end its trajectory in an active cell due to the distribution of active cells. The trajectory of a long muon is more likely to end in an inactive cell. The tracker places the end of the track in the last active cell with energy deposition 
along the track's path. In the following cases, however, the real endpoint of the muon would not be accurately reconstructed:

1. The muon could have ended its path in the region between planes with active cells.

2. The muon could have passed a plane with active cells through the PVC.

3. The tracker could have missed a cell hit in an active plane.

4. The muon could have left the detector before reaching the next active plane.

About $30 \%$ of the muons exhibit one of these behaviors [103]. In case (1), shown in figure 4.20, the muon stops at the end of the dotted path in red. On average, however, a muon with this topology will have an endpoint halfway between the end of the dotted path and the blue " $x$ ", as illustrated by the magenta line segment added to the end of the reconstructed track (red). The length of this segment extends the track to a point half the distance $(H)$ between the adjacent active planes, so that $H / 2$ is added to the $Z$ coordinate of the track's endpoint. With this correction, the difference between the true and reconstructed endpoint is, on average, centered at zero, and exhibits a resolution of $25 \mathrm{~cm}$, or the thickness of 5 cells [103].

The other three cases are more difficult to accurately reconstruct. Sample events of the last three cases are presented in figure 4.21, where top views for each event are labeled: Reco for the reconstructed track, and MC for the true trajectory. In case (2), illustrated in figure 4.21a, the true muon trajectory passes through the plastic (MC top view) of the active plane, around $Z=825 \mathrm{~cm}$, deposits no energy in that plane, and then stops around $Z=970 \mathrm{~cm}$. The reconstructed track (Reco top view) ends in the previous active plane, which represents a loss of about a third of the muon's reconstructed energy. In case (3), illustrated in figure 


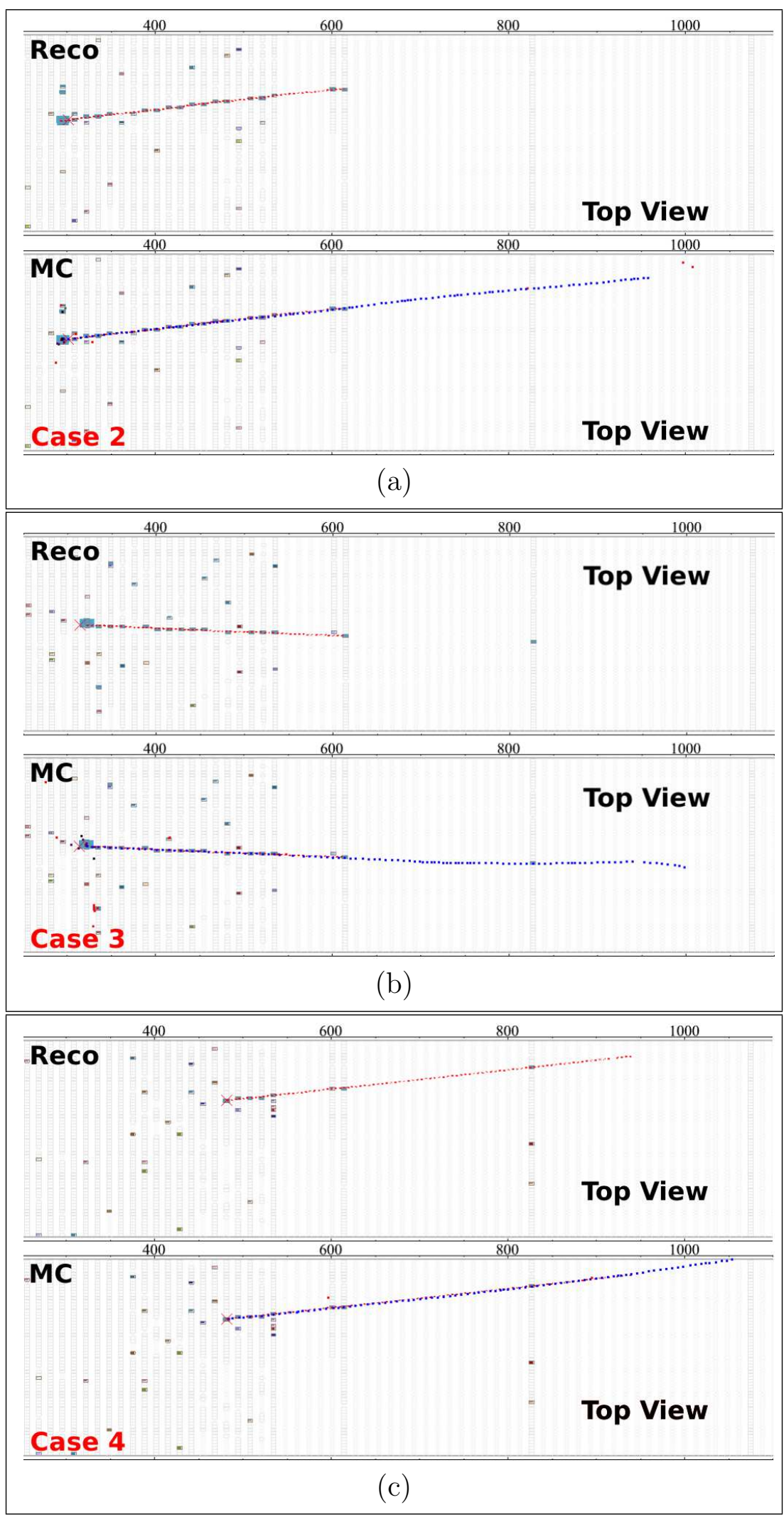

Figure 4.21 Sample Events. Cases: (a) 2, (b) 3, and (c) (4). Only the top view of the detector in each event is shown. The red tracks are reconstructed objects, and the blue tracks are the corresponding true objects. MC simulation. 
$4.21 \mathrm{~b}$, the tracker does not include in the muon track the cell hit left in the active plane around $Z=825 \mathrm{~cm}$, making the reconstructed red track around $3 \mathrm{~m}$ shorter than its true length, and its energy about $600 \mathrm{MeV}$ too low. In case (4), illustrated in figure 4.21c, the true muon trajectory leaves the detector (MC top view), at $Z=1050 \mathrm{~cm}$, before reaching the next active plane at $Z=1075 \mathrm{~cm}$. With this topology, the tracker incorrectly assumes the muon stops in the detector, which results in an energy estimation that is too low. These three cases are not properly represented by the correction applied to the track length in case (1), nonetheless, these will be corrected by the method described above since, in data, it is not possible to make a distinction from case (1).

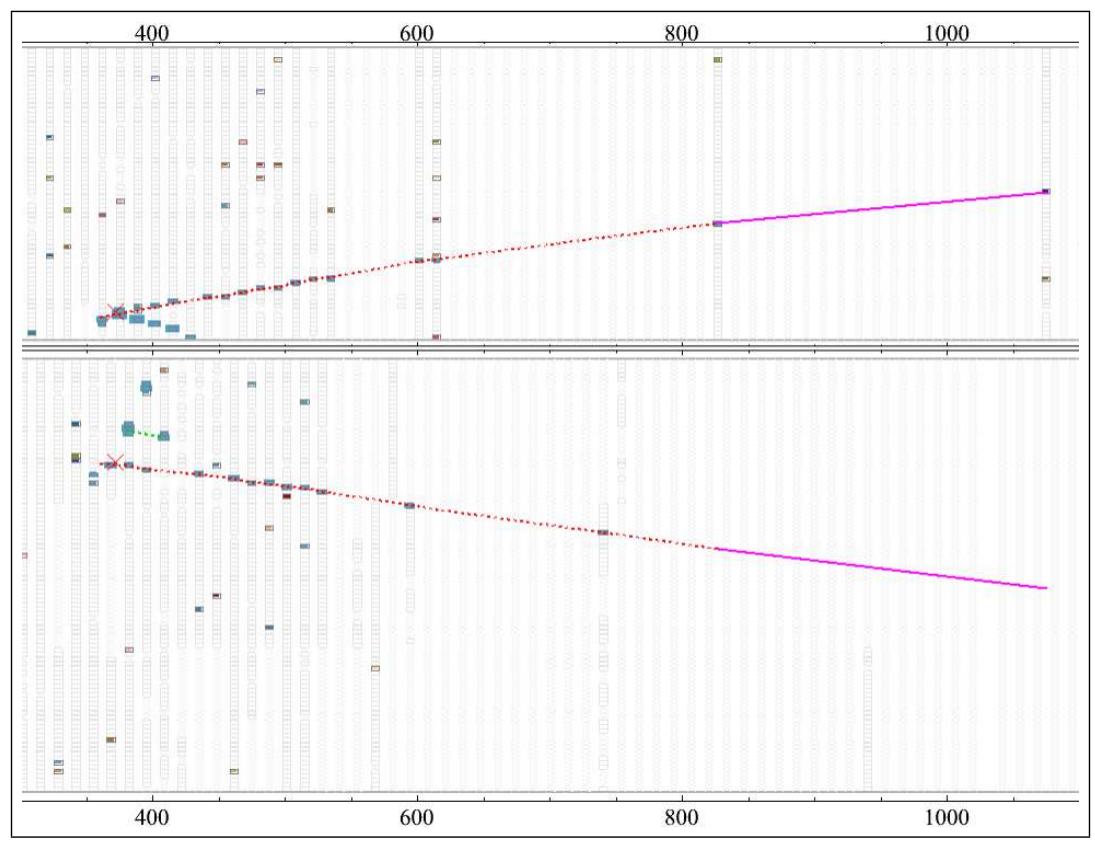

Figure 4.22 Sample Event. From figure 4.20 with extra track length correction applied for containment purposes. MC simulation.

For tracks that actually leave the detector (not contained), the length correction presented above is insufficient to obtain the correct energy. Therefore, tracks with the potential to leave the detector will be removed from the sample. The correction presented above is applied to 
the reconstructed track length of muons to better estimate, on average, the energy of the muon sample. The lack of information on the real endpoints of the muon tracks requires further manipulation of the track lengths in order to determine their containment. Only for containment purposes, an extra line segment is added to the muon tracks, with endpoints beyond $Z>600 \mathrm{~cm}$, so that the new reconstructed track length reaches the next active plane, as seen in figure 4.22. The magenta line segment goes from the last cell hit in the track to the next active plane in the projected muon trajectory. If the new endpoint of the extension (magenta line segment) is within the detector volume in both views, the track will be labeled as contained, while the uncontained tracks will be removed from the sample.

\subsection{Data Quality}

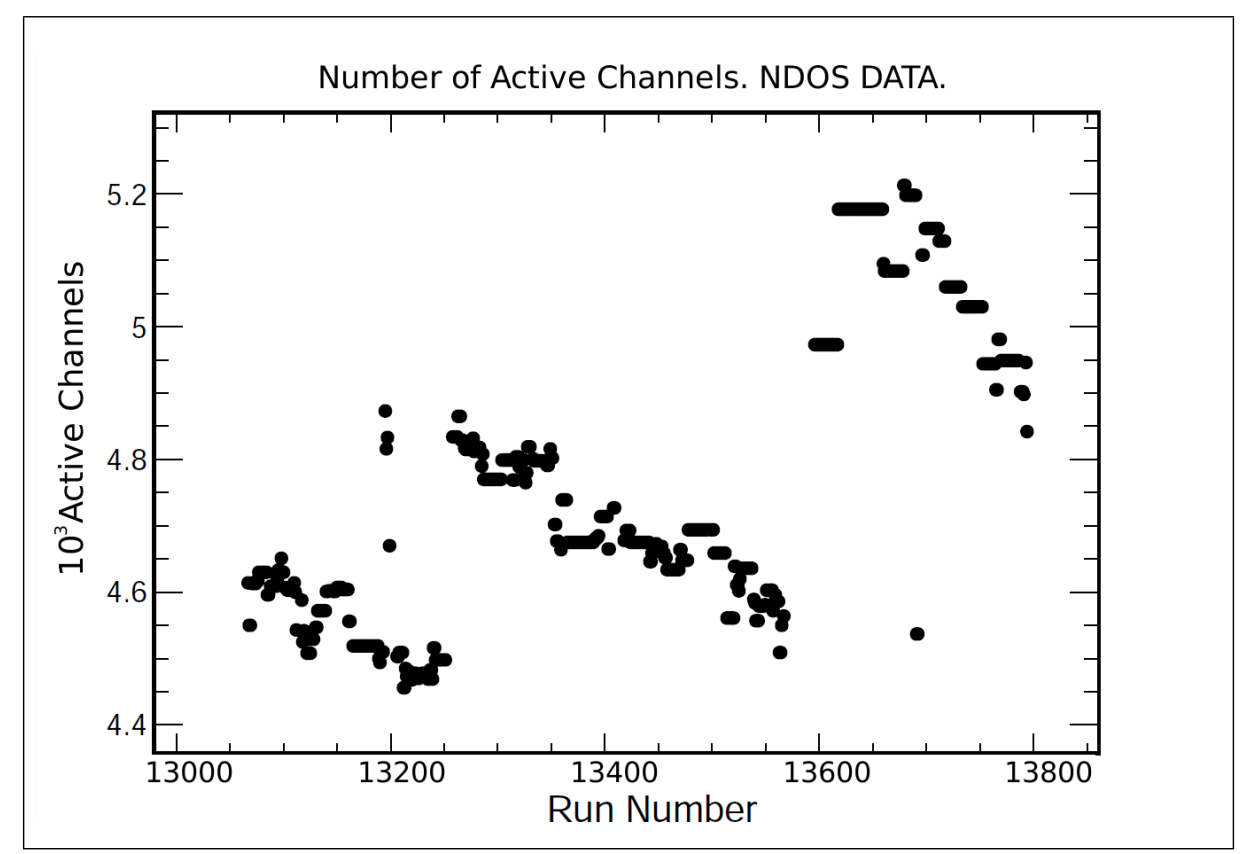

Figure 4.23 Number Of Active Channels Per Good Run. The good Run number increases with time: $13067=10 / 29 / 2011$, and $13782=04 / 30 / 2012$.

A number of quality checks are performed to make a list of good data files to be used in 
the physics analyses. A good data file, or SubRun, meets the following criteria:

- The first SubRun of a Run is longer than $30 \mathrm{~min}$.

- The file is not a collection of empty events.

- The file has less than $10 \%$ of noise-only events.

- The number of cell hits per active channel per event $\left(h_{\text {ace }}\right)$ in the file is: $0<h_{\text {ace }}<0.06$

- The average number of slices $\left(n_{s}\right)$ in the file is: $1<n_{s}<6$.

- The average slice duration $\left(t_{s}\right)$ in the file is: $0<t_{s}<1500 \mathrm{~ns}$.

- The file shows a record of synchronized DCMs.

Also, good data files must have at least 4400 active channels, as seen in figure 4.23.

In the midst of changing conditions of the NuMI beam, the reconstruction process yields a total number of neutrino candidate events that correlates well with the integrated number of POT [104], as shown in figure 4.24. Although the proton beam intensity varied throughout the data taking period, the beamline components did not change. There are a few times during the data taking period where the number of candidate events found jumped or dropped slightly in a small region of integrated POT. The candidate event rate: $2 \times 10^{-17}$ events/POT, correlates well with that expected on the basis of the neutrino beam energy spectrum and the cross sections contained within the simulation program.

The DAQ reads out cell hits per event in $500 \mu$ s wide trigger time windows centered at about the time of an expected neutrino arrival. The average time of the cell hits per physics 


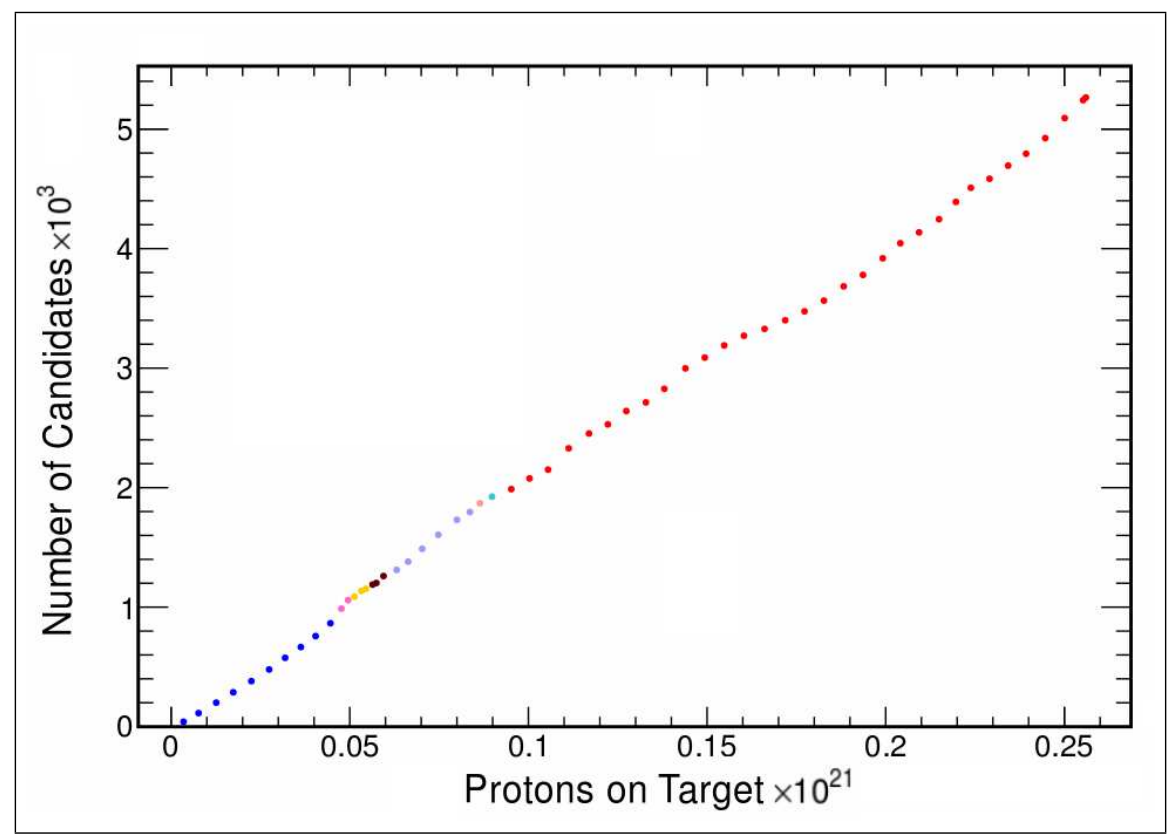

Figure 4.24 Number Of Neutrino Candidates As A Function Of The POT. Colors represent various beam configurations [104].

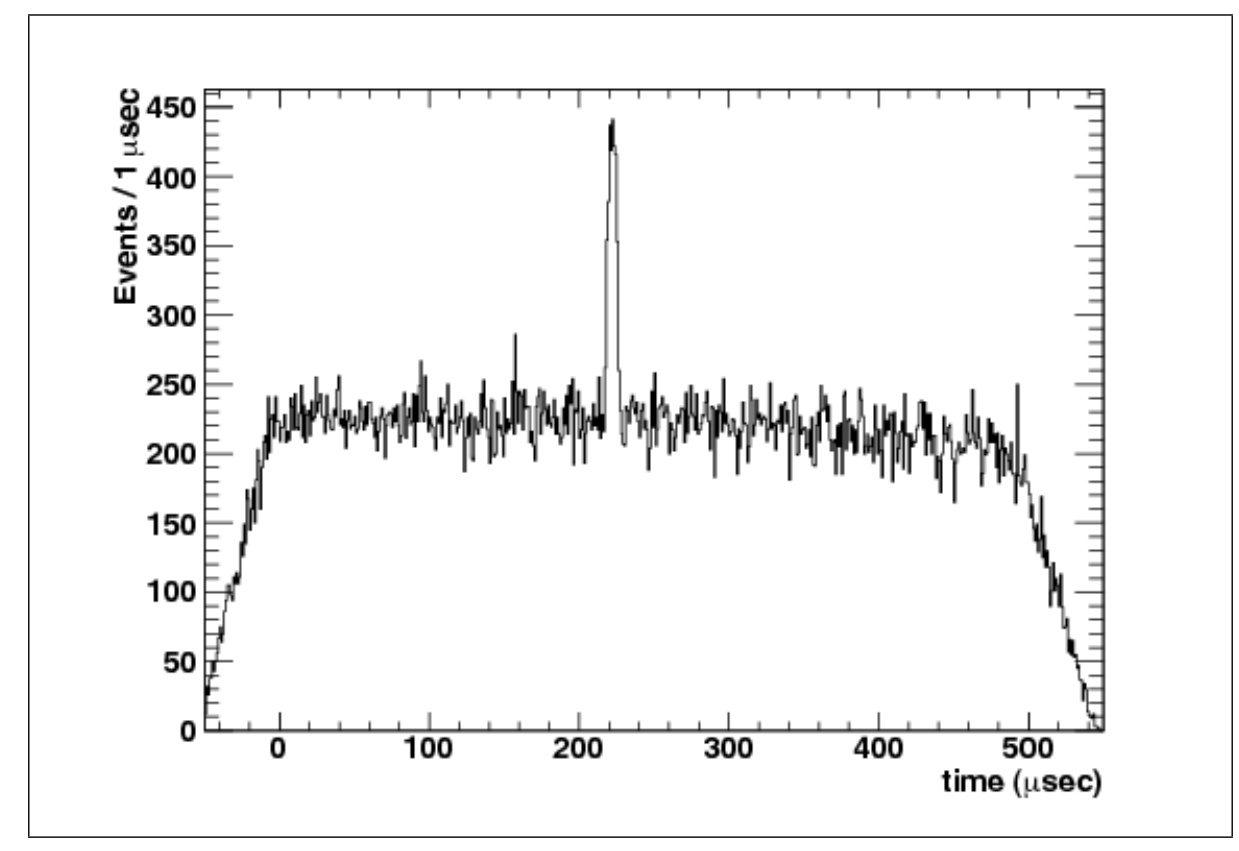

Figure 4.25 Time Of Event Slice In The Data Of The NO $\nu$ A Prototype Detector. Full DAQ window. Official NO $\nu$ A NDOS figure [105]. The bin size is $1 \mu \mathrm{s}$. 
slice is shown in figure 4.25 [105], where a physics slice is considered an event ${ }^{8}$. In the event display, the trigger time window is represented by a histogram that starts at $-50 \mu$ s and ends at $+550 \mu \mathrm{s}$. The data are read in blocks of $50 \mu \mathrm{s}$, and the boundaries of these blocks generally do not match the boundary of the $500 \mu \mathrm{s}$ time window, i.e. a portion of the first and last blocks belongs to the $500 \mu$ s time window, and the other portion does not. Two full blocks, one at the beginning and one at the end of the $500 \mu$ s time window, are presented in each event display. The amount of hits recorded in these two blocks falls rapidly for times away from the boundaries of the $500 \mu$ s time window. The neutrinos from the NuMI beam are expected within the beam spill, $10 \mu \mathrm{s}$ long, centered at $222 \mu \mathrm{s}$ into the trigger time window. A trigger time window containing a selected neutrino candidate event is shown in figure 4.26. The red arrow points to the peak of cell hits that comes from the selected neutrino candidate event at about $220 \mu \mathrm{s}$.

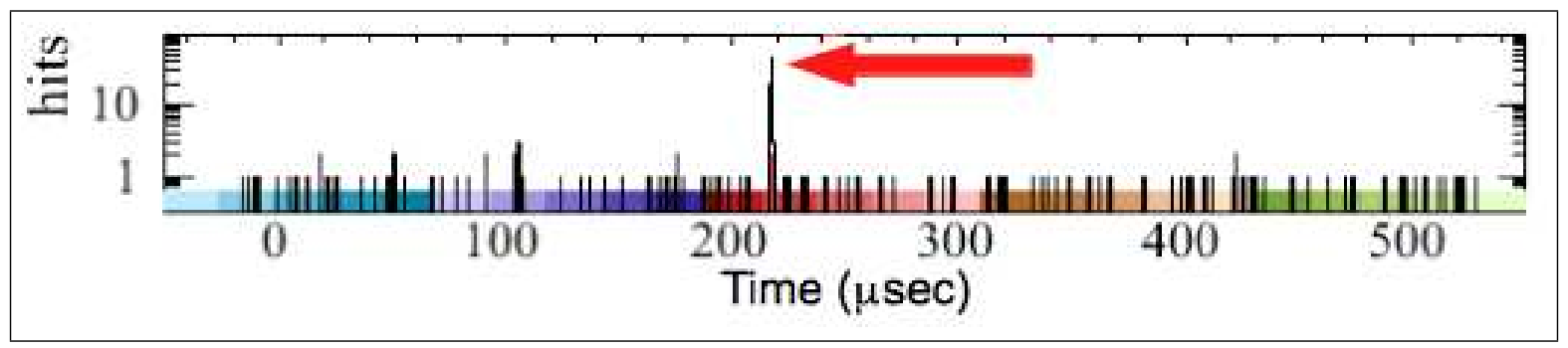

Figure 4.26 Sample Trigger Time Window. A selected neutrino candidate event with no cosmic ray background.

The out-of-time NDOS data, all from cosmic rays, which is defined by (refer to figure

${ }^{8}$ The word event used before to refer to collections of cell hits occurring within $500 \mu \mathrm{s}$ is also used to refer to a physics slice which is suppose to represent the cell hits of a neutrino interaction. 
4.25) the time intervals ${ }^{9}$ :

$$
t<+216.5 \mu \mathrm{s} \text {, and } t>+227.5 \mu \mathrm{s},
$$

has a constant rate in the time intervals:

$$
-3.5 \mu \mathrm{s}<t<+216.5 \mu \mathrm{s} \text {, and }+227.5 \mu \mathrm{s}<t<+436.5 \mu \mathrm{s} \text {. }
$$

The recorded cosmic ray rate rapidly goes to zero for:

$$
t<-3.5 \mu \mathrm{s} \text {, and } t>+436.5 \mu \mathrm{s} .
$$

The neutrino signal from the NuMI beam appears at about $222 \mu$ s in figure 4.25 , as expected. As discussed above, the beam spill interval is $10 \mu$ s wide. However the NDOS timing resolution is $0.5 \mu \mathrm{s}$, and consequently the in-time window is defined by:

$$
+216.5 \mu \mathrm{s}<t<+227.5 \mu \mathrm{s},
$$

a window that is $11 \mu$ s wide for this NDOS analysis. 


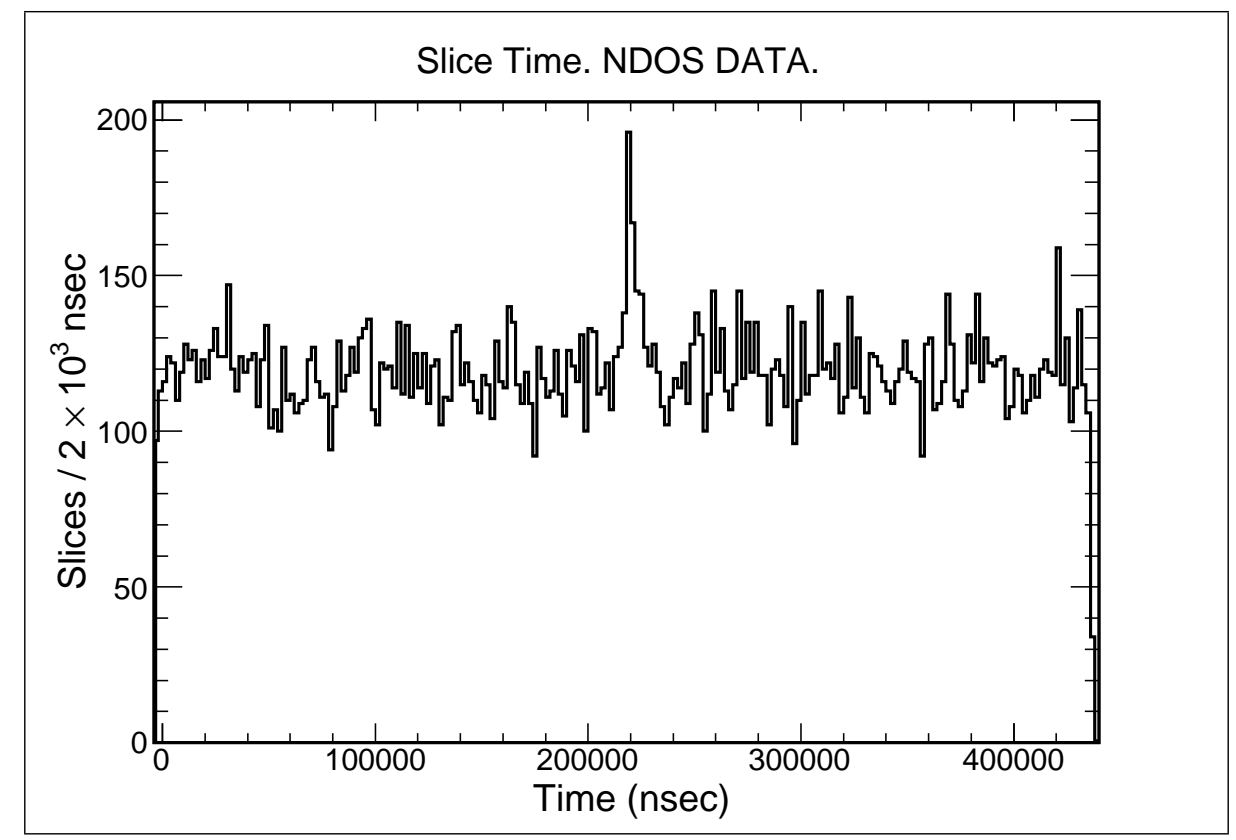

Figure 4.27 Time Of Physics Slices In The Data Of The Prototype Detector. Note the in-time data at about $220 \mu \mathrm{s}$.

\subsection{Cosmic Rays}

As a consequence of its location in a surface building, NDOS records millions of cosmic rays.

A constant rate of cosmic rays is recorded in each trigger window for:

$$
-3.5 \mu \mathrm{s}<t<+436.5 \mu \mathrm{s}
$$

Therefore, to establish the level of cosmic background in the neutrino data, cosmic rays within this time interval, $429 \mu$ s wide $^{10}$, are considered. The time interval used to establish the constant cosmic rate is shown in figure 4.27. The bin size is $2 \mu \mathrm{s}$, four times the NDOS

\footnotetext{
${ }^{9}$ These time intervals exclude the beam window.

${ }^{10}$ Note that the width of $429 \mu$ s results from the exclusion of the in-time data, which is $11 \mu$ s wide.
} 
timing resolution ${ }^{11}$. A straight line fit to the out-of-time data allows to determine that the cosmic ray rate is flat within the shown time interval. The parameters of the fit are:

$$
\begin{aligned}
\text { y-intercept: } & (114.9 \pm 1.5) \text { Slices } / 2 \times 10^{3} \mathrm{~ns}, \\
\text { slope: } & \left(\left(5.5 \times 10^{-7}\right) \pm\left(6.1 \times 10^{-6}\right)\right) \text { Slices } / 2 \times 10^{3} \mathrm{~ns}^{2} .
\end{aligned}
$$

The slope is consistent with zero, and its variation over the range of the time window is of $0.2 \%$. The timing histogram representing the trigger window of an event with four reconstructed cosmic rays is shown in figure 4.28. The peaks coming from their cells hits appear at about: $55 \mu \mathrm{s}, 90 \mu \mathrm{s}, 290 \mu \mathrm{s}$, and $380 \mu \mathrm{s}$. Note that there is no peak of hits at the NuMI trigger time, around $222 \mu \mathrm{s}$. The event display of this particular event with cosmic rays is presented in figure 4.29 .

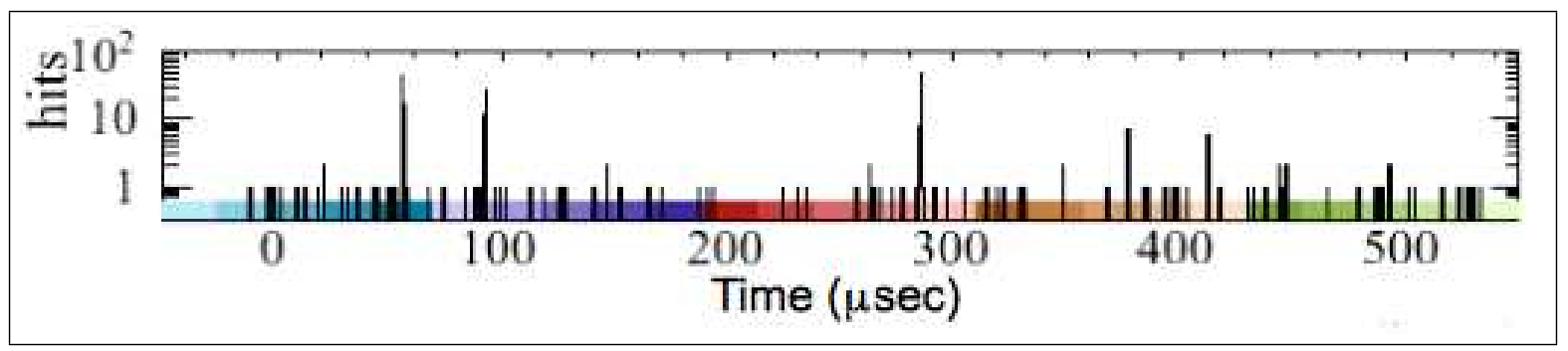

Figure 4.28 Sample Trigger Time Window. Only cosmic rays in the event.

In order to reduce the cosmic ray background that appears inside the NuMI trigger window, the angular distribution of the out-of-time cosmic tracks is compared to that from the MC simulated beam tracks. Three angles are studied: $\cos \theta_{X}, \cos \theta_{Y}$, and $\cos \theta_{\mathrm{NuMI}}$. The first two angles are those between the unit vectors of the detector's axes $X$ and $Y(\hat{i}$

${ }^{11}$ All the slices that passed the containment cuts discussed in section 5.2, and had a reconstructed track with track length longer than $2 \mathrm{~m}$ are presented in figure 4.27 . The time assigned to a slice is an average of all its cell hits times. 


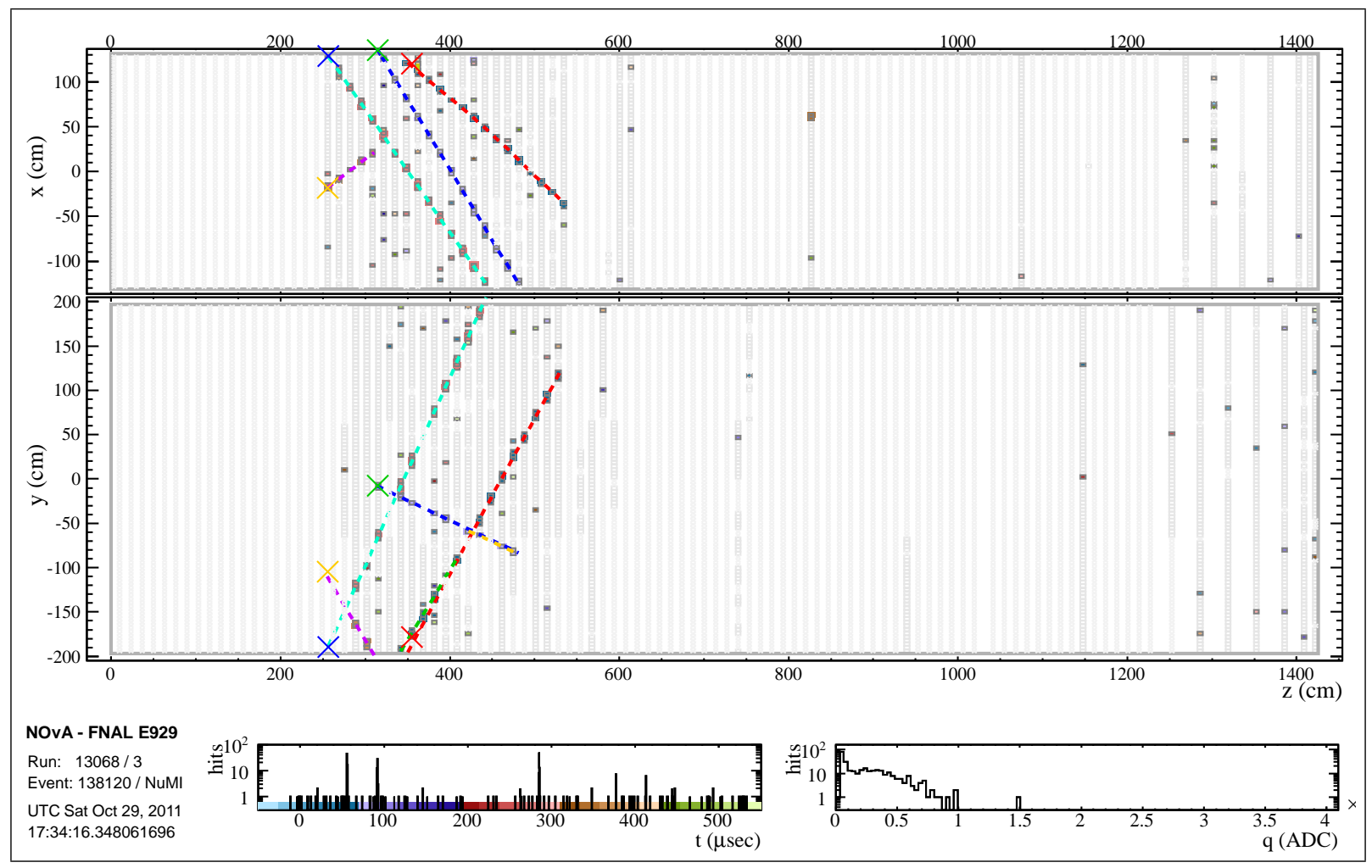

Figure 4.29 Sample Cosmic Ray Event. This figure shows a sample cosmic ray event with four reconstructed cosmic rays. 


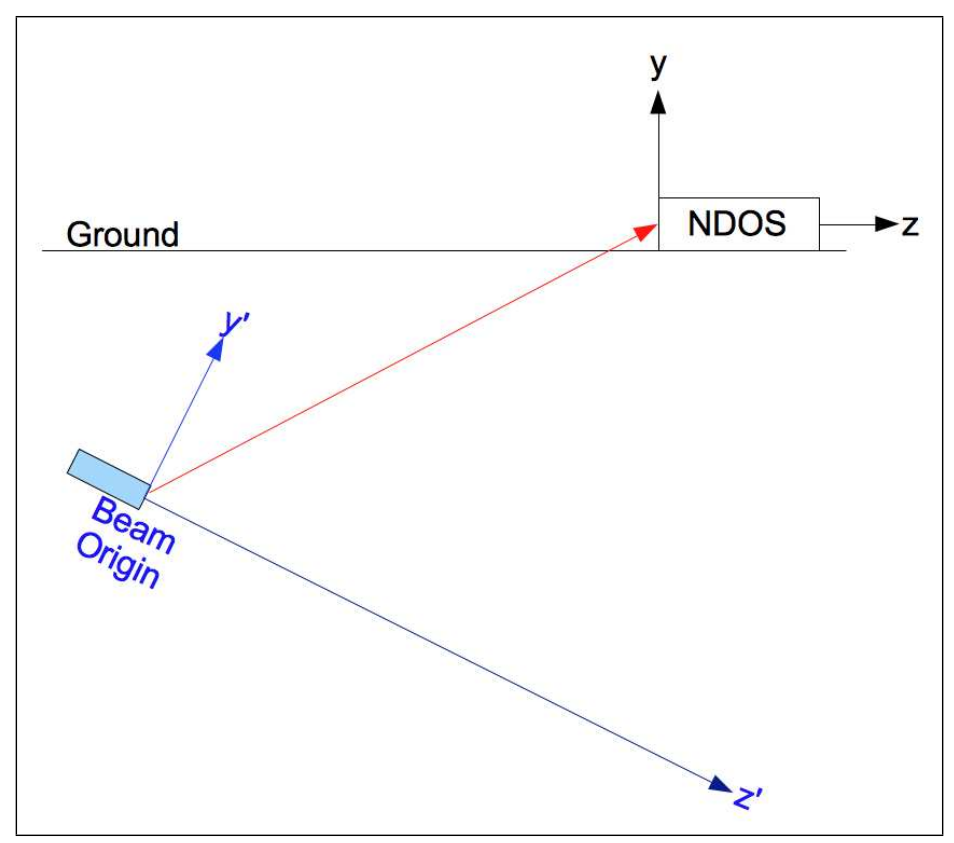

Figure 4.30 Beam To Prototype Detector Coordinate Transformation Illustration. Graphical representation of the beam reference frame (blue) and the NDOS reference frame (black). The red arrow represents the direction of the neutrinos, from the NuMI beam, that reach the NDOS. Not to scale.

and $\hat{j}$ ), and the unit vector of the longest track in the event, $\hat{v}_{t}$ :

$$
\begin{aligned}
& \hat{i} \cdot \hat{v}_{t}=\cos \theta_{X}=\frac{X_{f}-X_{i}}{L}, \\
& \hat{j} \cdot \hat{v}_{t}=\cos \theta_{Y}=\frac{Y_{f}-Y_{i}}{L}, \\
& \hat{k} \cdot \hat{v}_{t}=\cos \theta_{Z}=\frac{Z_{f}-Z_{i}}{L},
\end{aligned}
$$

where $X_{i}, Y_{i}$, and $Z_{i}$ are the starting point coordinates of the longest track in the event; $X_{f}$, $Y_{f}$ and $Z_{f}$ are the ending point coordinates of the track, and $L$ is the track's length. The $\cos \theta_{\mathrm{NuMI}}$ is the cosine of the angle between $\hat{v}_{t}$ and the unit vector of the neutrinos coming 
from the NuMI beam, $\hat{v}_{N}$ :

$$
\begin{aligned}
\hat{v}_{N} \cdot \hat{v}_{t} & =\cos \theta_{\mathrm{NuMI}}=v_{N}^{x} v_{t}^{x}+v_{N}^{y} v_{t}^{y}+v_{N}^{z} v_{t}^{z}, \\
& =v_{N}^{x} \cos \theta_{X}+v_{N}^{y} \cos \theta_{Y}+v_{N}^{z} \cos \theta_{Z}, \\
& =\frac{d x}{d s} \cos \theta_{X}+\frac{d y}{d s} \cos \theta_{Y}+\frac{d z}{d s} \cos \theta_{Z} \\
& =\frac{d z}{d s}\left(\frac{d s}{d z} \frac{d x}{d s} \cos \theta_{X}+\frac{d s}{d z} \frac{d y}{d s} \cos \theta_{Y}+\cos \theta_{Z}\right), \\
& =\frac{1}{\frac{d s}{d z}}\left(\frac{d x}{d z} \cos \theta_{X}+\frac{d y}{d z} \cos \theta_{Y}+\cos \theta_{Z}\right)
\end{aligned}
$$

The origin of the NDOS reference frame is: $d x^{\prime}=-0.29 \mathrm{~m}, d y^{\prime}=92.21 \mathrm{~m}$, and $d z^{\prime}=841.76 \mathrm{~m}$, with $d s^{\prime}=846.80 \mathrm{~m}$, in the beam coordinates. The coordinates transformation derived in [106] (see figure 4.30) allows to get that point in the NDOS reference frame: $d x=-29.0 \mathrm{~cm}$, $d y=4300.8 \mathrm{~cm}$, and $d z=78070.2 \mathrm{~cm}$, with $d s=78188.6 \mathrm{~cm}$. In the NDOS reference frame: $\frac{d s}{d z}=1.00151, \frac{d x}{d z}=0.00037$, and $\frac{d y}{d z}=0.055$. From equation (4.15), the $\cos \theta_{\mathrm{NuMI}}$ is:

$$
\cos \theta_{\mathrm{NuMI}}=\frac{0.055 \cos \theta_{Y}+\cos \theta_{Z}}{1.00151}
$$

The ratio $\frac{d x}{d z}$ is assumed to be zero in the MC simulation as it is small compared to the other fractions. The direction of $\hat{v}_{N}$ is illustrated in figure 4.31 .

Cosmic rays are a background to the neutrino signal. However the angle of a cosmic ray muon is rarely parallel to the direction of the neutrino beam. The angular distribution of the longest tracks in the simulated CC neutrino interactions are peaked in the direction of the neutrino beam, as shown in figure 4.32. The cosmic rays and the simulated tracks from neutrino events have peaks at: $\cos \theta_{X}=0$, as shown in figure $4.32 \mathrm{a}$, so that this variable does not provide much discrimination power. Cosmic ray tracks have a strong preference for the 


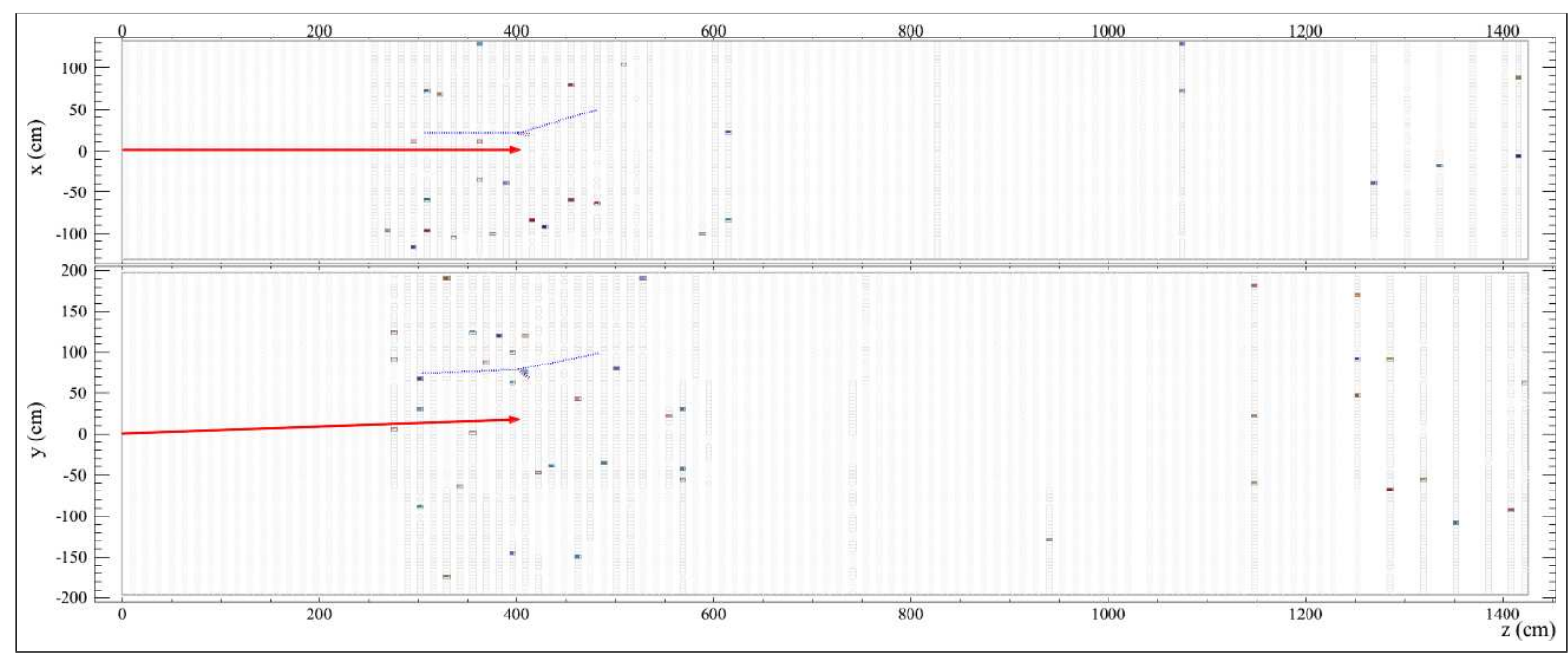

Figure 4.31 The Direction Of Neutrinos From The NuMI Beam In The NDOS. The direction of the neutrinos from the NuMI beam at the NDOS location is represented by red arrows drawn on top of the NDOS event display.

vertical direction, $\left|\cos \theta_{Y}\right| \approx 1$, while long tracks generated in neutrino interactions prefer the horizontal direction, $\cos \theta_{Y} \sim 0$, as shown in figure $4.32 \mathrm{~b}$. The greatest discrimination between cosmic rays and the longest tracks from neutrino interactions is in the angle with respect to the beam direction. The neutrino interaction tracks have a peak at: $\cos \theta_{\mathrm{NuMI}} \approx 1$, in contrast with the maximum exhibited by the cosmic rays at: $\cos \theta_{\mathrm{NuMI}} \approx 0.1$, as shown in figure $4.32 \mathrm{c}$.

Simple cuts in the angular distributions of the reconstructed tracks can make a clean separation between neutrino data and the cosmic ray background, as will be discussed in Chapter 5. All the reconstruction tools presented in this chapter aid to analyze the data that passes the quality control checks summarized here. 


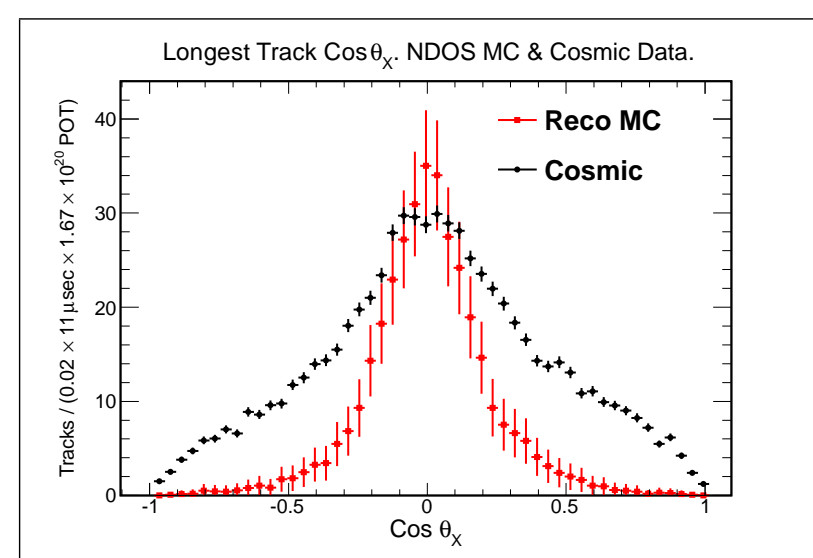

(a)
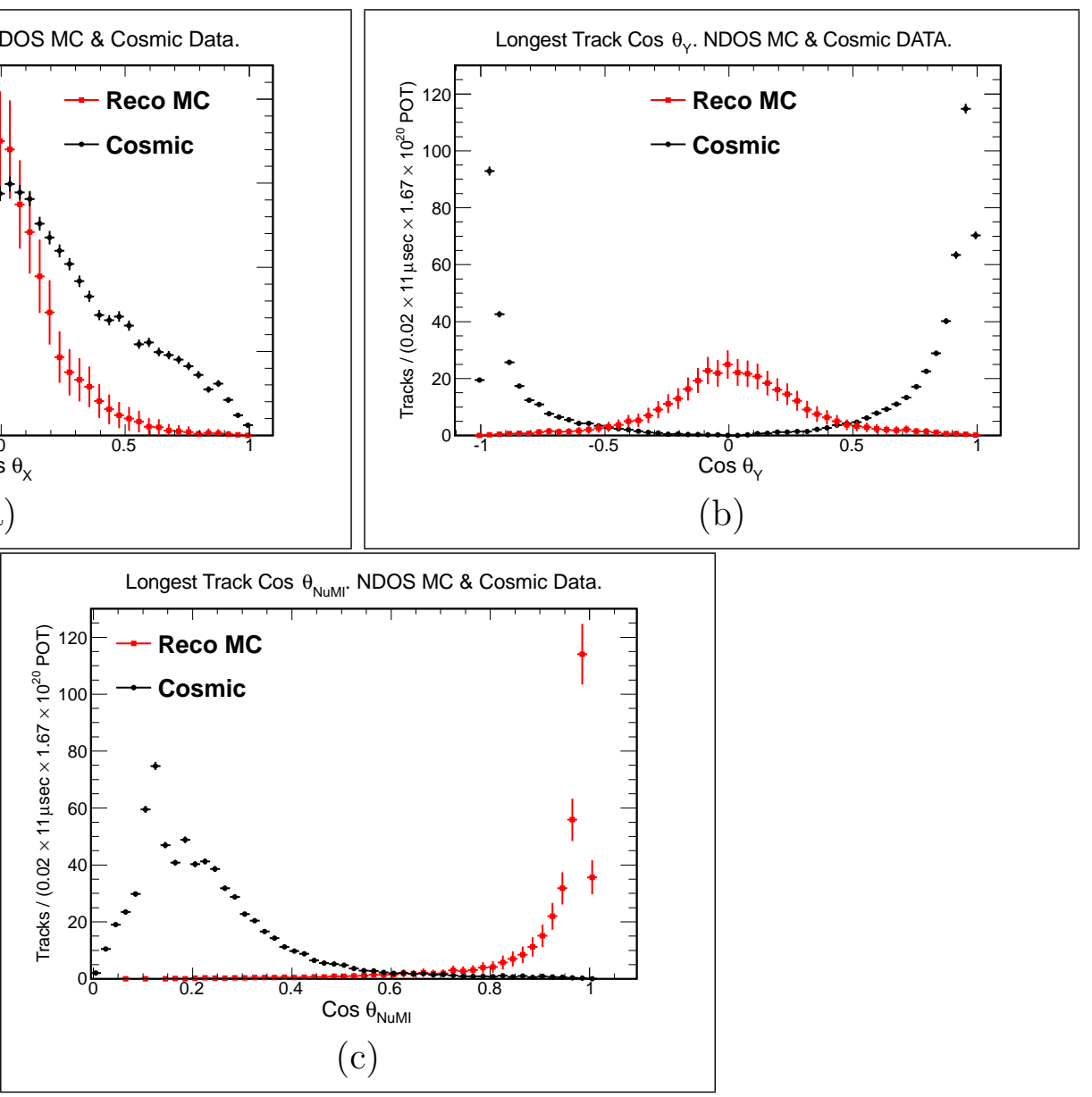

Figure 4.32 Longest Track Angular Distributions. NDOS MC (red) and cosmic ray data (black). Longest track MC and out-of-time cosmic data (a) $\cos \theta_{X}$, (b) $\cos \theta_{Y}$, and (c) $\cos \theta_{\text {NuMI }}$ distributions. 


\section{Chapter 5}

\section{Event Selection}

As discussed in section 4.4, an event is a set of data collected within a $500 \mu$ s time window; however, many different physical processes can occur within this time. The purpose of event selection is to identify probable neutrino interactions within this time window, and study them in order to determine their nature, i.e. their interaction type ( $\mathrm{CC}$ or $\mathrm{NC})$, and the neutrino type $\left(\nu_{\mu}\right.$ or $\left.\nu_{e}\right)$. In the NDOS, the word event refers to a neutrino interaction without confusion since the chances of two or more neutrino interactions in one $500 \mu \mathrm{s}$ window are extremely low.

Although muons are easy to identify in the $\mathrm{NO} \nu \mathrm{A}$ detectors, energetic charged pions and protons that behave like minimum ionizing particles (MIP) represent a background to the muon sample ${ }^{1}$. When protons and charged pions undergo hard scattering, these leave in the detector characteristic signatures, figures 5.1a and 5.1c are examples of these signatures. These two simulated single particle events have momenta around $2 \mathrm{GeV} / \mathrm{c}$, and do not represent a background to the muon signal because they are either too short or too heavily ionizing. In contrast, when protons and charged pions behave like a MIP, as seen in figures $5.1 \mathrm{~b}$ and $5.1 \mathrm{~d}$, these do represent a background to the muon signal. These two simulated single particle events also have momenta around $2 \mathrm{GeV} / \mathrm{c}$, however, these leave different signatures in the detector, similar to that of muons. Hadrons with momenta around

\footnotetext{
${ }^{1}$ Electromagnetic showers also contribute, in smaller proportions, to the background.
} 


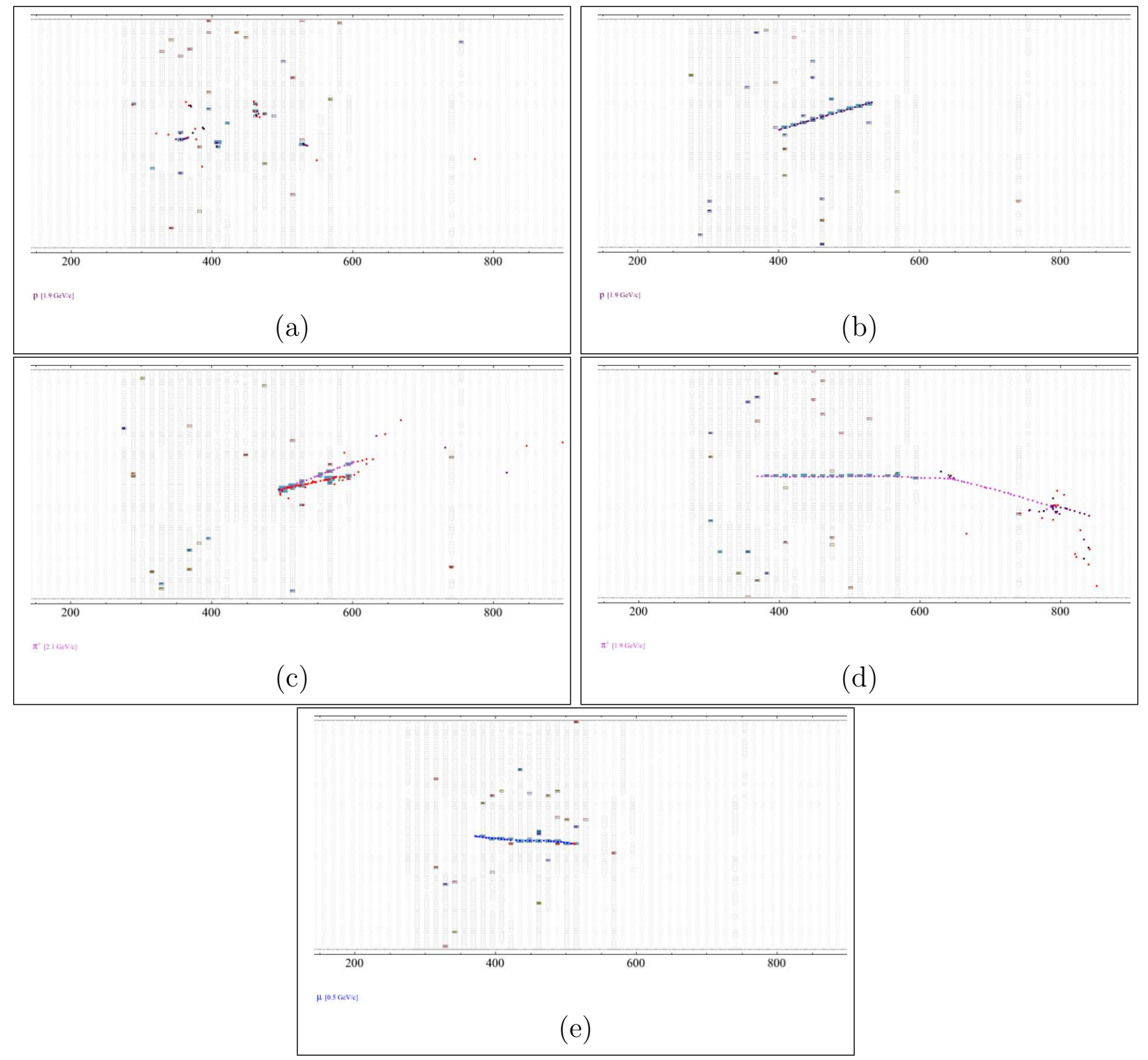

Figure 5.1 Single Particle Events. (a) Proton: $1.9 \mathrm{GeV} / \mathrm{c}$. (b) Proton: $1.9 \mathrm{GeV} / \mathrm{c}$. (c) $\pi^{+}: 2.1 \mathrm{GeV} /$ c. (d) $\pi^{+}: 1.9 \mathrm{GeV} /$ c. (e) $\mu: 0.5 \mathrm{GeV} /$ c. Colored dots represent the various energy depositions left in the detector by the particles. MC simulation. 
$2 \mathrm{GeV} / \mathrm{c}$ that behave like a MIP are not as much of a background to $2 \mathrm{GeV} / \mathrm{c}$ muons as these are to $0.5 \mathrm{GeV} / \mathrm{c}$ muons, as seen in figure 5.1e.

Described in the sections to follow are the cuts applied to reject events without a final state muon. The various efficiencies and purities of the selected sample are also presented in the following sections. The cuts that remove cosmic rays from the selected event sample are included as well.

\subsection{Charged And Neutral Current Neutrino Interac- tions}
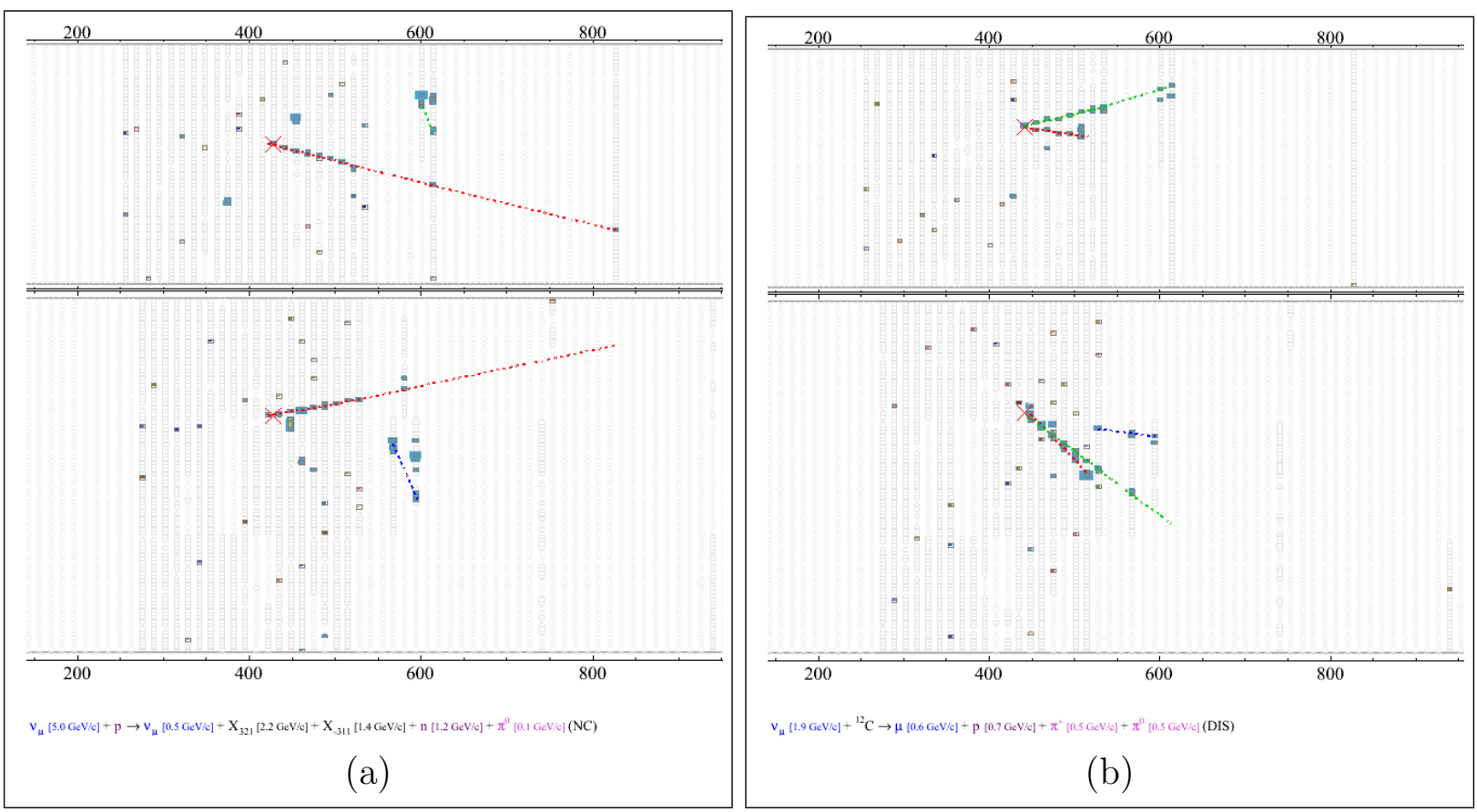

Figure 5.2 Sample Events. (a) Sample NC event: $\nu_{\mu}+p \rightarrow \nu_{\mu}+K^{+}+K^{0}+n+\pi^{0}$. (b) Sample CC event: $\nu_{\mu}+{ }^{12} \mathrm{C} \rightarrow \mu+p+\pi^{-}+\pi^{0}$. MC simulation.

$\mathrm{CC}$ neutrino interactions have a background that comes from $\mathrm{NC}$ neutrino interactions which contain a hadronic track that mimics a muon. Two sample MC events are shown in 
figure 5.2: one is a $\mathrm{NC}$ event (figure 5.2a), and the other one is a CC event (figure 5.2b). The two events in figure 5.2 show a similar energy deposition, and a long MIP track. A longest track with MIP behavior is shown in figure 5.2a; this particle is a $K^{+}$(red track). The event also features a $K^{0}$, a neutron and a $\pi^{0}$. The neutrino energy is $5.0 \mathrm{GeV}$. In contrast, figure $5.2 \mathrm{~b}$ has a muon as its longest track (green track). The event also has a $\pi^{0}$ that decays into two photons, a proton, and a $\pi^{+}$. This is a DIS event. The neutrino energy is $1.9 \mathrm{GeV}$. Figure 5.2a is an example of a NC MC simulated event that needs to be rejected in order to achieve a clean CC sample. Section 5.3 is going to address the rejection criteria.

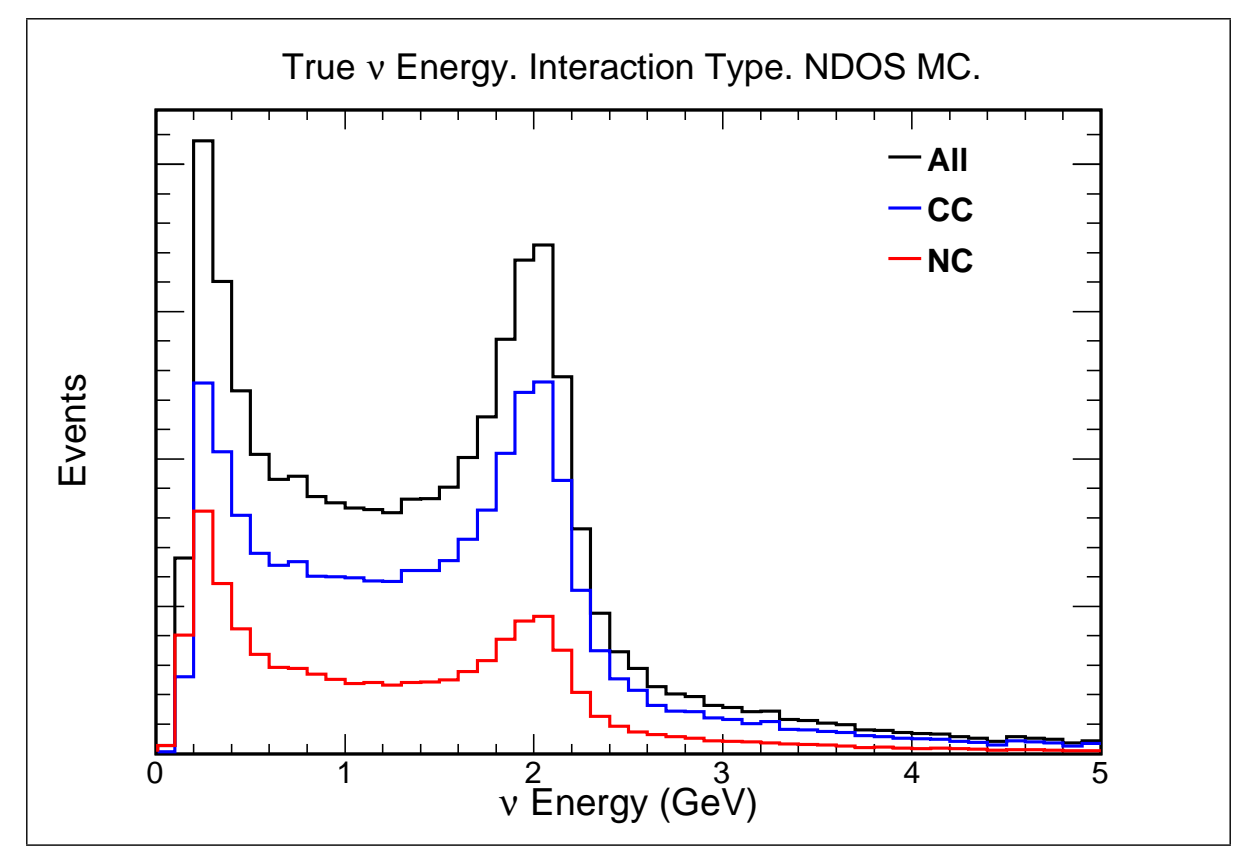

Figure 5.3 NDOS Neutrino Energy Distributions. Predicted neutrino energy distributions discriminated by interaction type. All interactions (black), CC (blue), and NC (red) energy distributions. MC simulation.

From the MC simulation it is known that $69.4 \%$ of the neutrino interactions in the NDOS are $\mathrm{CC}$ and $30.6 \%$ are $\mathrm{NC}$, as seen in figure 5.3. The $\frac{\mathrm{CC}}{\mathrm{NC}}$ ratio rises rapidly with neutrino energy, as shown in figure 5.4. For energies above $1.8 \mathrm{GeV}$ the ratio is about 2.6, consistent with measured cross sections ratios [44]. 


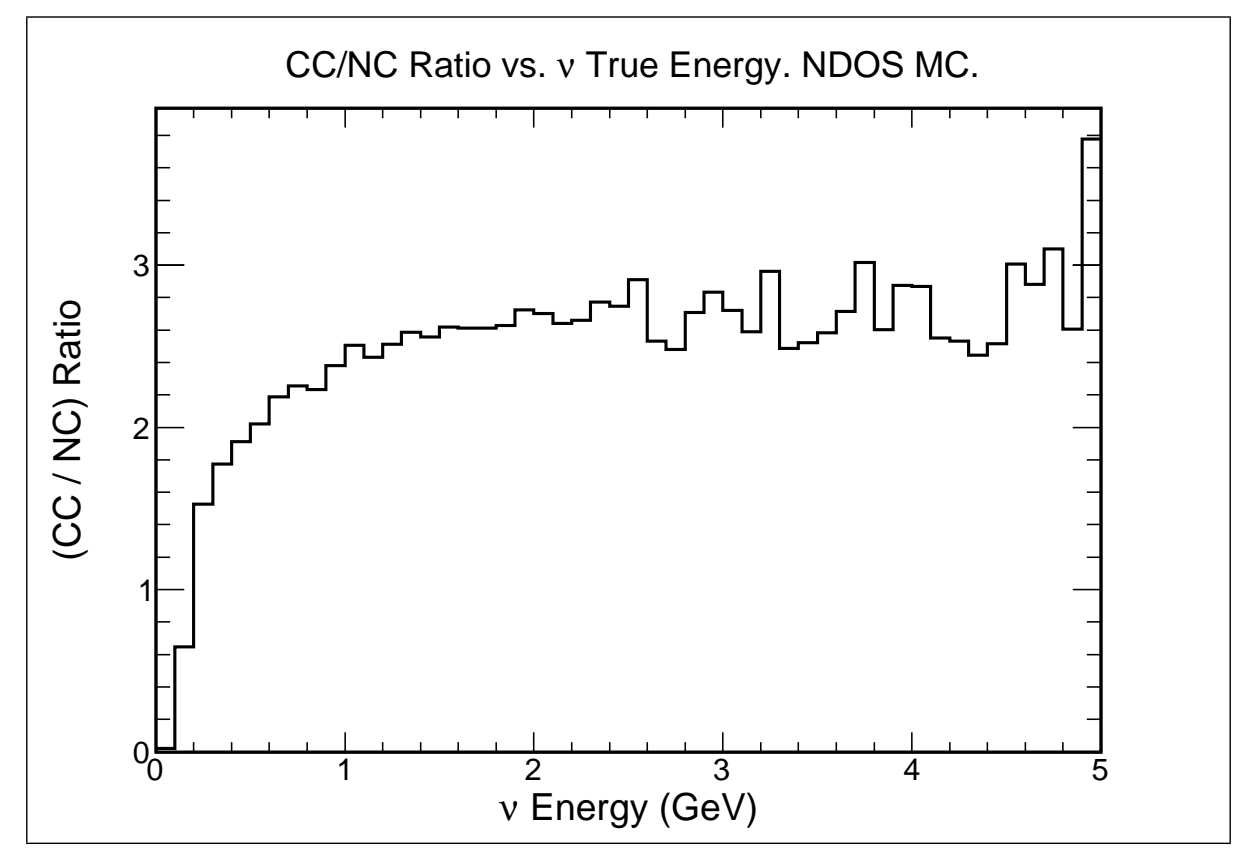

Figure 5.4 MC Prediction Of The Ratio Of CC To NC Neutrino Interactions. Ratio as a function of neutrino energy for all simulated events.

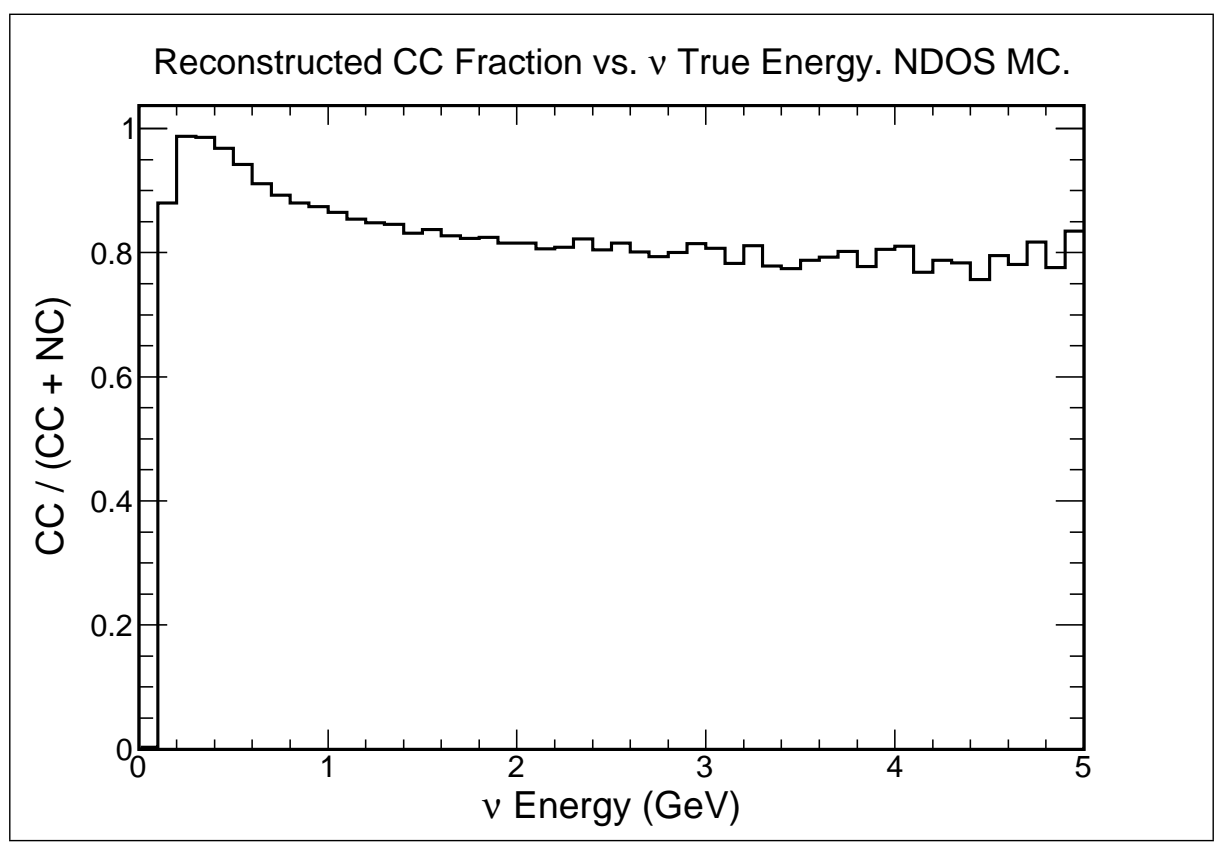

Figure 5.5 Fraction of Reconstructed CC Events. Fraction of reconstructed CC events out of the total sample: $\mathrm{CC}+\mathrm{NC}$, with at least one reconstructed track. MC simulation. 


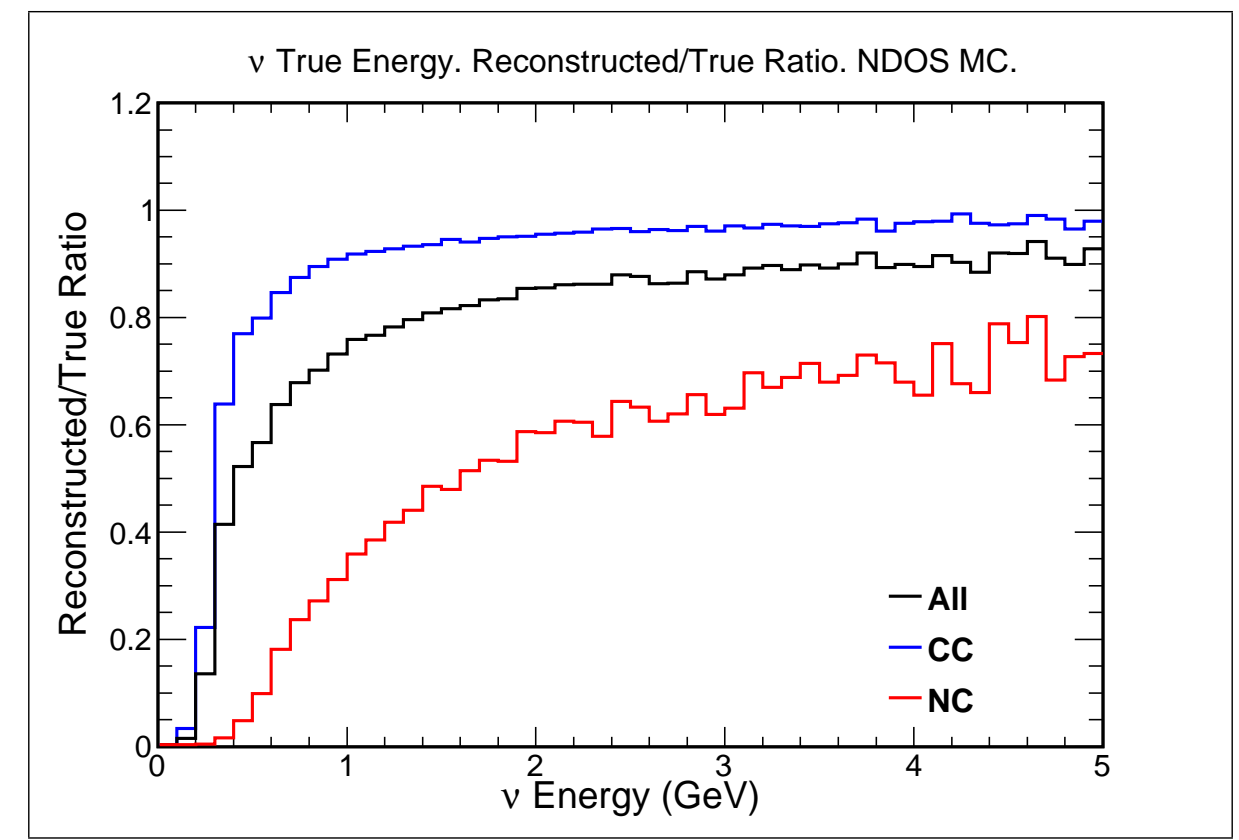

Figure 5.6 Ratio Of Reconstructed Over Simulated Events. Reconstruction ratio as a function of the predicted neutrino energy discriminated by interaction type. The ratio reconstructed/true for: all interactions (black), $\mathrm{NC}$ (red), and CC (blue) energy distributions. All reconstructed events must have at least one reconstructed track. MC simulation.

As presented in section 4.3.2.2, the requirement of a minimum of 8 hits to reconstruct a 3D track is a strong cut resulting in an efficiency that strongly favors the desired CC events, as shown in figure 5.5. At neutrino energies less than $0.1 \mathrm{GeV}$, no events are reconstructed. For energies above $0.1 \mathrm{GeV}$ the $\mathrm{CC}$ sample with at least one reconstructed track is about four times larger than the reconstructed NC sample. From the total number of reconstructed events: $81.4 \%$ are CC and $18.6 \%$ are NC. Within this sample there are three ratios of interest: all reconstructed events to all simulated events, all reconstructed CC events to all simulated $\mathrm{CC}$ events, and all reconstructed $\mathrm{NC}$ events to all simulated $\mathrm{NC}$ events, as seen figure 5.6. Events with energies less than $0.1 \mathrm{GeV}$ are not reconstructed by the reconstruction algorithms due to the lack of information. The $\mathrm{NC}$ event reconstruction rate drops below $50 \%$ for event energy less than $1.8 \mathrm{GeV}$, and the $\mathrm{CC}$ reconstruction rate dramatically drops 
for event energy below $0.4 \mathrm{GeV}$. Summarizing, $26.4 \%$ of all simulated interactions are not reconstructed. The effects of the remaining NC background is discussed in section 5.3.

\subsection{Event Containment Criteria}

Reconstructed neutrino interactions are required to originate ${ }^{2}$ in the vertex region (VR) defined as (refer to figure 5.7):

$$
\begin{gathered}
|X|<106 \mathrm{~cm}, \\
|Y|<172 \mathrm{~cm}, \\
288 \mathrm{~cm}<Z<452 \mathrm{~cm} .
\end{gathered}
$$

These VR limits are chosen such that the number of neutrino interactions occurring in it is maximum while the purity ${ }^{3}$ and efficiency ${ }^{4}$ of the limits are maximum as well. The VR limits also ensure that full cell transverse areas are included.

The VR represents $8 \%$ of the total NDOS volume. The instrumented regions in front (lower $Z$ ) and to the sides of the limits of the VR are used as veto regions in order to ensure that the longest track of each event starts within the VR. Hits within these veto regions imply that the track came from outside the VR. The reconstructed neutrino interaction 3D coordinates, or vertex, are defined at the point with lowest $Z$ coordinate of the longest track. The simulation indicates that the containment of the reconstructed interaction vertex within

\footnotetext{
${ }^{2}$ See section 4.2 .

${ }^{3}$ See equation (5.2).

${ }^{4}$ See equation (5.3).
} 


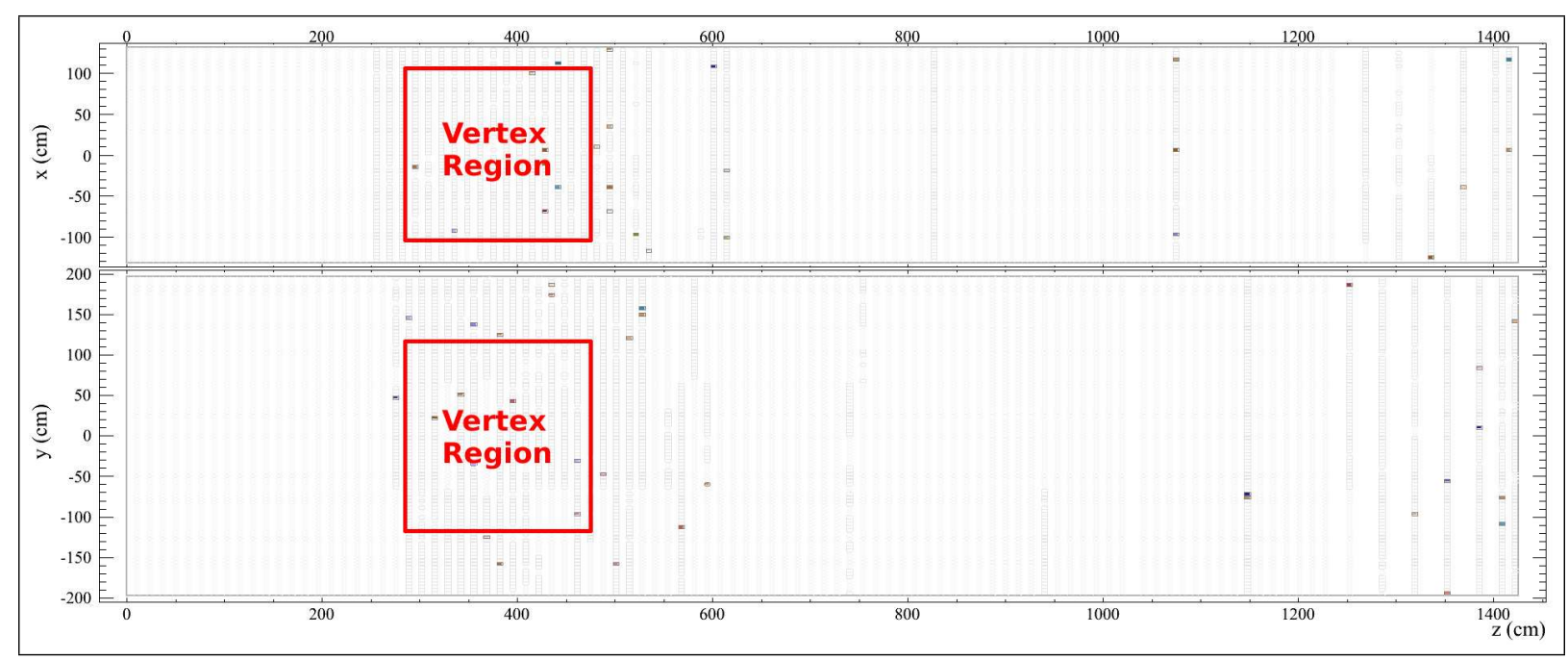

Figure 5.7 Vertex Region Of The Prototype Detector.

the VR has a purity (p) of:

$$
\mathrm{p}=\frac{N_{R}}{N_{T}}=(96.7 \pm 0.2) \%
$$

where $N_{R}$ is the number of reconstructed tracks starting within the VR that have a corresponding true track starting within the VR, and $N_{T}$ is the total number of reconstructed tracks starting within the VR. The efficiency $(\epsilon)$ is:

$$
\epsilon=\frac{N_{R}}{N_{e}}=(95.6 \pm 0.2) \%
$$

where $N_{e}$ is the number of true tracks starting within the VR. The VR purity and efficiency as a function of neutrino energy are summarized in table 5.1, at the end of this chapter.

To determine the energy of a muon it must range out and stop in the detector, therefore it has to be contained within the detector. Containment of the longest track is also one of the cuts designed to reduce background from cosmic ray muons. The longest track is required 


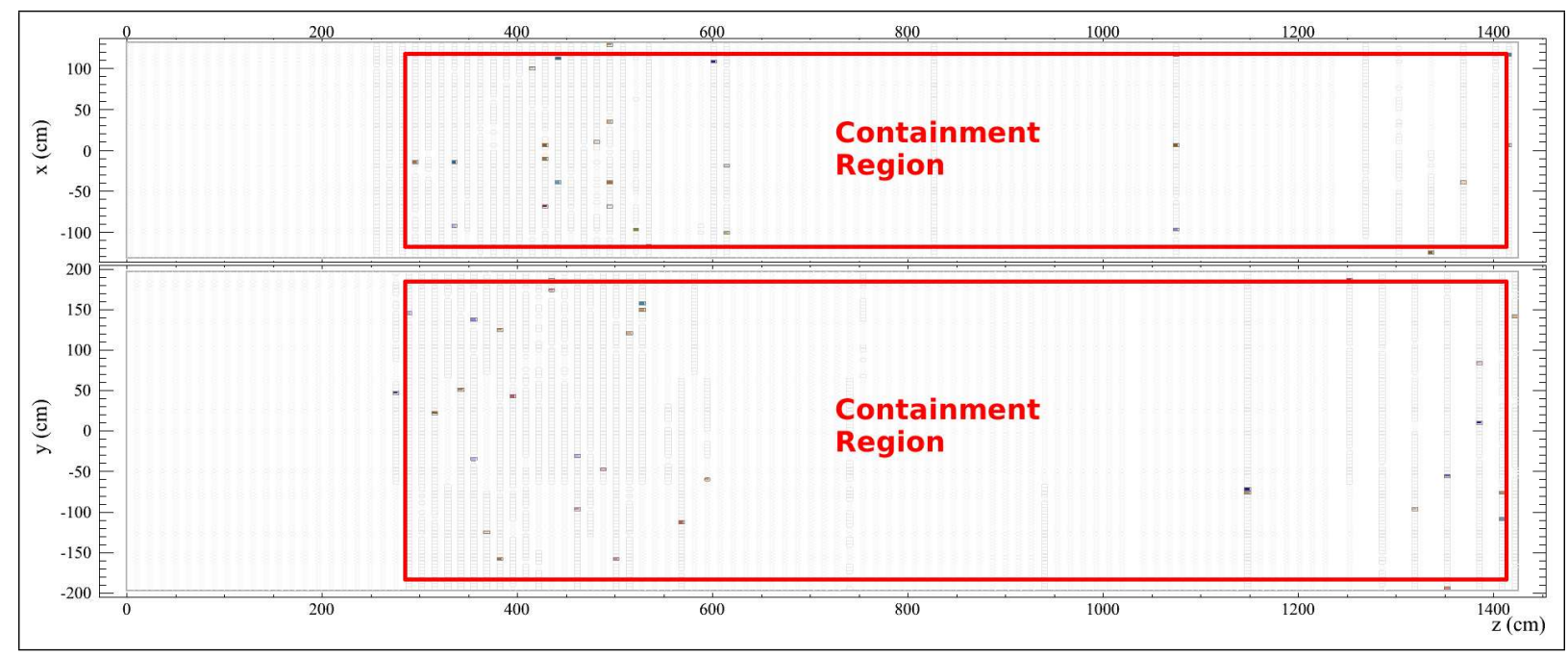

Figure 5.8 Containment Region Of The Prototype Detector.

to be contained within a containment region (CR) defined as (refer to figure 5.8):

$$
\begin{gathered}
|X|<119 \mathrm{~cm} \\
|Y|<184 \mathrm{~cm} \\
288 \mathrm{~cm}<Z<1406 \mathrm{~cm} .
\end{gathered}
$$

These CR limits are chosen such that the number of neutrino events contained in it is maximum while the purity ${ }^{5}$ and efficiency ${ }^{6}$ of the limits are maximum as well. The CR limits also ensure that full cell transverse areas are included. In order for a longest track to be fully contained, this has to start within the VR and end within the CR. Outside the CR boundaries there are three veto cells between the edges of the CR and the edges of the detector $^{7}$. Hits in these veto cells imply that the track is not fully contained, and therefore

\footnotetext{
${ }^{5}$ See equation $(5.5)$.

${ }^{6}$ See equation (5.6).

${ }^{7}$ From figure 5.8 it is clear that between $Z=0$ and $Z=288 \mathrm{~cm}$ there are more than three cells. All the instrumented volume in this region is used as veto.
} 
the particle left the detector; or it came from outside the detector. The simulation indicates that the containment of the longest track within the CR has a purity (p) of:

$$
\mathrm{p}=\frac{N_{R}}{N_{T}}=(87.7 \pm 0.4) \%
$$

where $N_{R}$ is the number of reconstructed tracks contained within the CR that have a corresponding true track contained within the $\mathrm{CR}$, and $N_{T}$ is the total number of reconstructed tracks contained within the CR. The efficiency $(\epsilon)$ is:

$$
\epsilon=\frac{N_{R}}{N_{e}}=(76.1 \pm 0.5) \%
$$

where $N_{e}$ is the number of true tracks contained within the CR. The CR purity and efficiency as a function of neutrino energy are summarized in table 5.1, at the end of this chapter. These purities and efficiencies are affected by the small number of active planes beyond the VR that are used to identify if, and where, the muon stopped within the detector.

To verify the containment of a reconstructed longest track within the VR and the CR the track must be 3D. The simulation indicates that the efficiency $(\epsilon)$ of reconstructed $3 \mathrm{D}$ tracks is:

$$
\epsilon=\frac{N_{R}}{N_{e}}=(79.6 \pm 0.2) \%
$$

where $N_{R}$ is the number of reconstructed $3 \mathrm{D}$ tracks with an associated true track, and $N_{e}$ is the total number of true tracks. The efficiencies as a function of neutrino energy are summarized in table 5.1, at the end of this chapter. The low number of active channels outside the $\mathrm{VR}$ is responsible for the $20 \%$ of longest tracks that are only reconstructed as $2 \mathrm{D}$ tracks. In these cases, one of the views does not have enough information to reconstruct 
a 2D track, therefore, with only one $2 \mathrm{D}$ reconstructed track, a 3D reconstructed track is impossible to make.

\subsection{Charged Current Event Selection}

In order to identify the $\mathrm{CC}$ neutrino events from the $\mathrm{NC}$ and cosmic ray backgrounds, the NDOS MC simulation is used find the physical variables that are most sensitive to the differences between them. To make such distinctions, various reconstructed physical variables are investigated.

\subsubsection{Longest Track Length Cut}

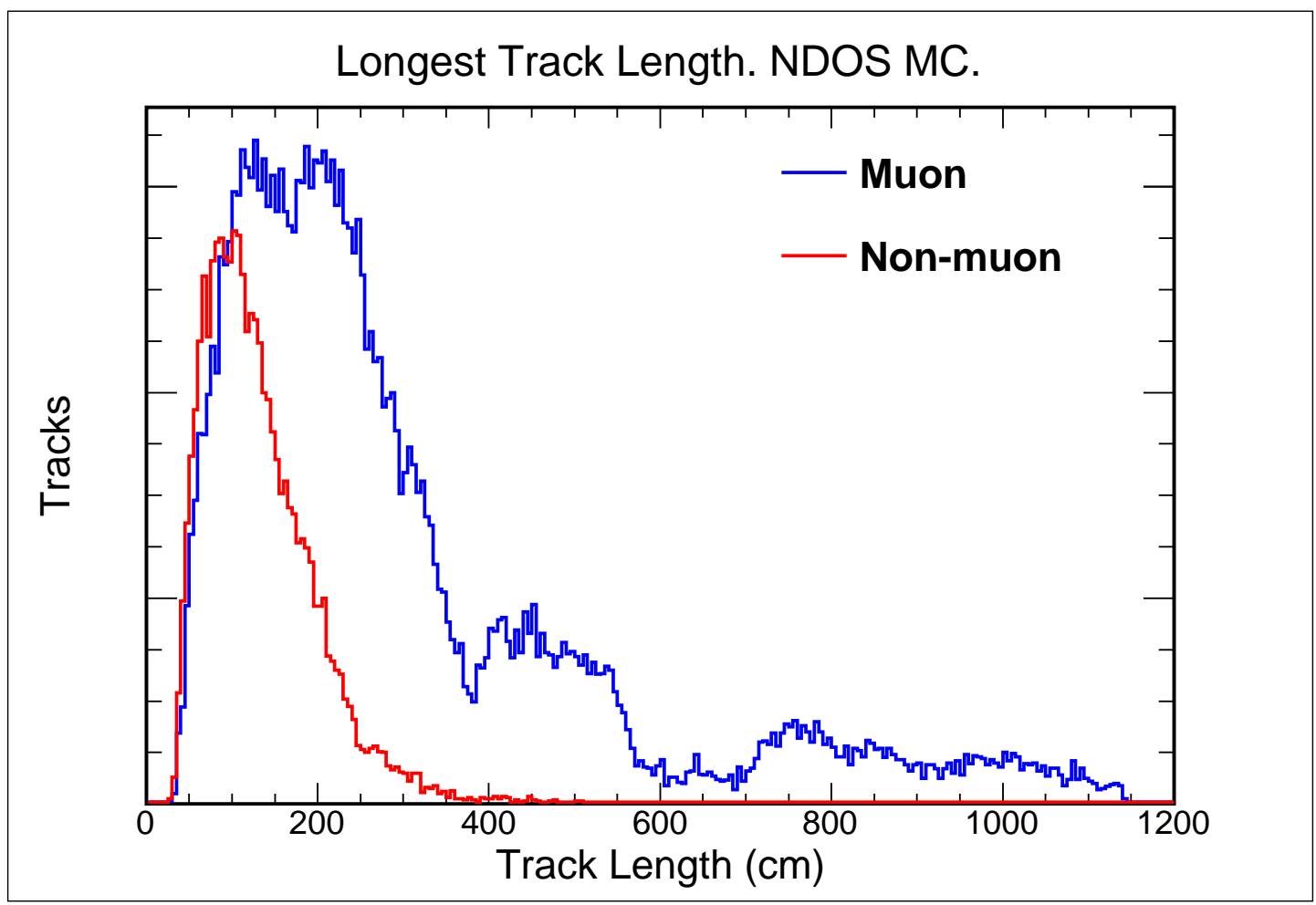

Figure 5.9 Monte Carlo Simulated Longest Track Length Distributions For Muons and Non-muons. Muon (blue), and non-muon (red) track length distributions. MC simulation. 
The main characteristic of a $\nu_{\mu} \mathrm{CC}$ event of interest is that it has a muon. In these $\mathrm{CC}$ events the longest track is typically that of the muon, and the longer the track, the lower is the background from hadronic or electromagnetic tracks (non-muon), as seen in figure 5.9. The next approach to identify $\mathrm{CC}$ events is to study the reconstructed longest track length (LTL) for each event, and separately examine CC and NC samples. These tracks must start within the VR and be 3D.

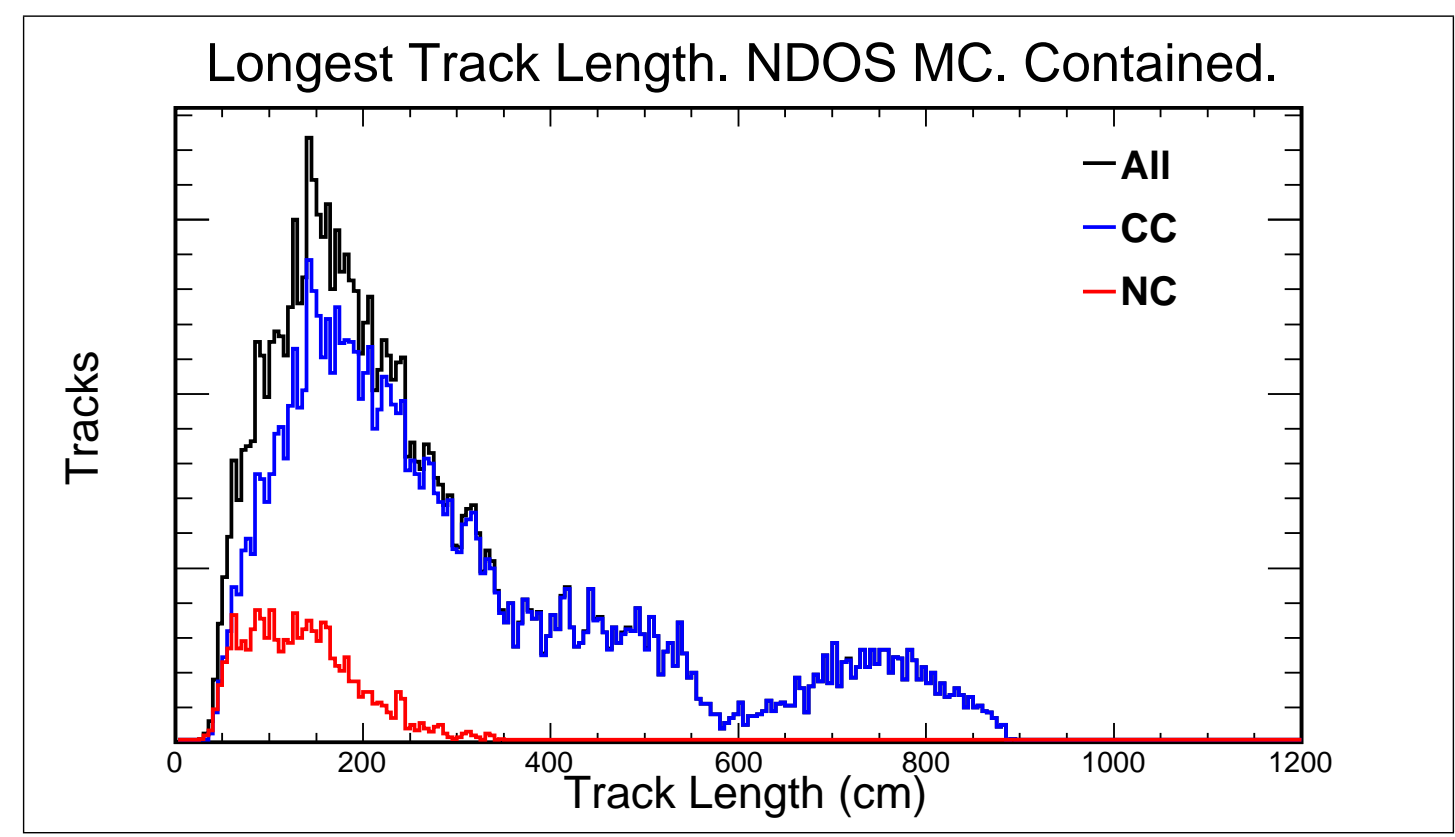

Figure 5.10 Monte Carlo Simulation Of The Longest Track Length Distributions. All interactions (black), CC (blue), and NC (red) longest track length distributions. All longest tracks are contained. MC simulation.

The LTL distributions are shown in figure 5.10 for each interaction type. Based on these distributions, a cut is chosen at:

$$
L T L>200 \mathrm{~cm} .
$$

Relation (5.8) divides the sample into two: the first one is that with $L T L>200 \mathrm{~cm}$, which has a $3.8 \%$ background coming from $\mathrm{NC}$ events, and the second one is that with $L T L<200 \mathrm{~cm}$, 
which has a $24.7 \%$ background coming from NC events. The contained reconstructed sample shows that $63.9 \%$ of all the CC reconstructed tracks, and $17.1 \%$ of all the $\mathrm{NC}$ reconstructed tracks are longer than $200 \mathrm{~cm}$. The analysis sample has all the $L T L>200 \mathrm{~cm}$ and it is assumed to be a CC sample with a $3.8 \%$ of $\mathrm{NC}$ background. The MC simulation indicates that the purity (p) of the track length cut (TLC) is:

$$
\mathrm{p}=\frac{N_{R}}{N_{T}}=(94.5 \pm 0.1) \%,
$$

where $N_{R}$ is the number of reconstructed tracks longer than $200 \mathrm{~cm}$ that have a corresponding true track longer than $200 \mathrm{~cm}$, and $N_{T}$ is the total number of reconstructed tracks longer than $200 \mathrm{~cm}$. The efficiency $(\epsilon)$ is:

$$
\epsilon=\frac{N_{R}}{N_{e}}=(59.6 \pm 0.3) \%,
$$

where $N_{e}$ is the number of true tracks longer than $200 \mathrm{~cm}$. The TLC purity and efficiency as a function of neutrino energy are summarized in table 5.1, at the end of this chapter. These relatively low efficiencies come from the combined effect of the reconstructed 3D tracks, the reconstructed end point of track, and contained tracks efficiencies.

\subsubsection{Cosmic Ray Cuts}

The containment cut on the longest track in an event removes a large fraction of the cosmic rays that are within the NuMI time window. However, those that appear to stop within the vertex region and slip through the containment cut can be mistaken for a CC neutrino event. 


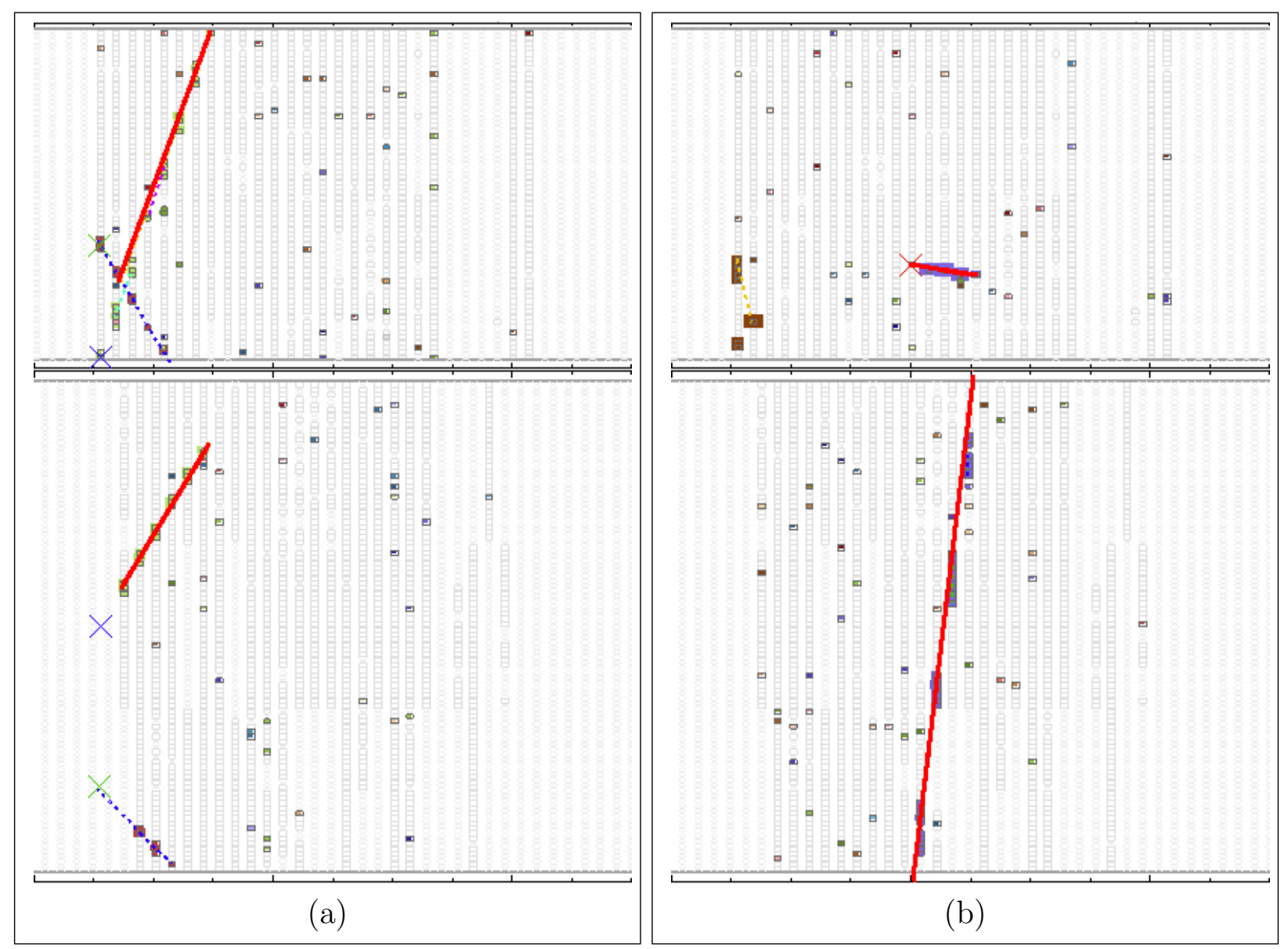

Figure 5.11 Cosmic Rays Rejection Zone By The Cut In $\cos \theta_{Y}$. Reconstructed cosmic tracks in red with (a) $\cos \theta_{Y}=0.6$, and with (b) $\cos \theta_{Y}=1.0$. 
Additional cuts on the angles of the longest track, discussed in section 4.5, are necessary to further reduce this background. In section 4.5 three cosines were defined. The $\cos \theta_{Y}$ distributions for MC simulation and out-of-time data (see figure 4.32b) present clear differences between signal and background. The longest track $\cos \theta_{Y}$ ratio: background/signal, becomes less than 1 for $\left|\cos \theta_{Y}\right|<0.6$. In order to reject cosmic rays, only in-time events with longest tracks with $\left|\cos \theta_{Y}\right|<0.6$ are accepted. This cut rejects $90.7 \%$ of the cosmic background, and keeps $94.4 \%$ of the neutrino data. The $\cos \theta_{\mathrm{NuMI}}$ distributions for $\mathrm{MC}$ simulation and out-of-time data (see figure 4.32c) present a clear difference between signal and background. The longest track $\cos \theta_{\mathrm{NuMI}}$ ratio: background/signal, becomes less than 1 for $\cos \theta_{\mathrm{NuMI}}>0.6$. To further reject cosmic rays, only in-time events with longest tracks with $\cos \theta_{\text {NuMI }}>0.6$ are accepted. This cut rejects $96.9 \%$ of the cosmic background, and keeps $96.3 \%$ of the neutrino data. The two cuts combined reject: $98.5 \%$ of all cosmic rays, and keep $93.4 \%$ of the neutrino data.

Two sample cosmic events that span the rejection region defined by the cut in $\cos \theta_{Y}$ are shown in figure 5.11. A cosmic ray with $\cos \theta_{Y}=0.6$ is shown in figure 5.11a, and a cosmic ray with $\cos \theta_{Y}=1.0$ is shown in figure 5.11b. Another two cosmic events that span the rejection region, defined by the cut in $\cos \theta_{\mathrm{NuMI}}$ are presented in figure 5.12. A cosmic ray with $\cos \theta_{\text {NuMI }}=0$ is shown in figure 5.12a, and a $\operatorname{cosmic}$ ray with $\cos \theta_{\text {NuMI }}=0.6$ is shown in figure $5.12 \mathrm{~b}$.

To summarize, the cosmic cuts accept tracks that fulfill:

$$
\left|\cos \theta_{Y}\right|<0.6, \quad \cos \theta_{\mathrm{NuMI}}>0.6 .
$$

The correlations between $\cos \theta_{Y}$ and $\cos \theta_{\text {NuMI }}$ are shown in figure 5.13. Most of the cosmic 

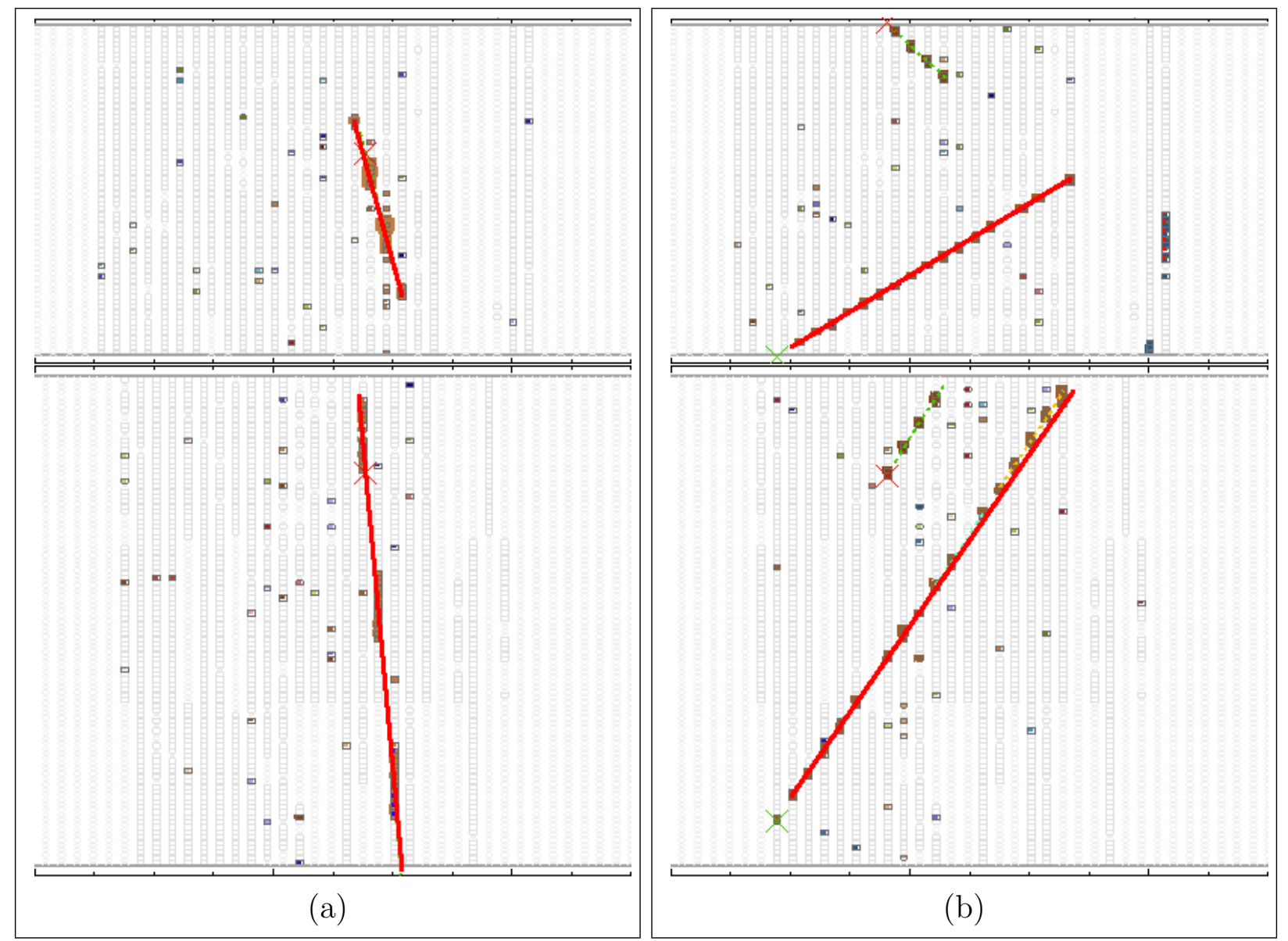

Figure 5.12 Cosmic Rays Rejection Zone By The Cut In $\cos \theta_{\text {NuMI }}$. Reconstructed cosmic tracks in red with (a) $\cos \theta_{\mathrm{NuMI}}=0$, and with (b) $\cos \theta_{\mathrm{NuMI}}=0.6$.

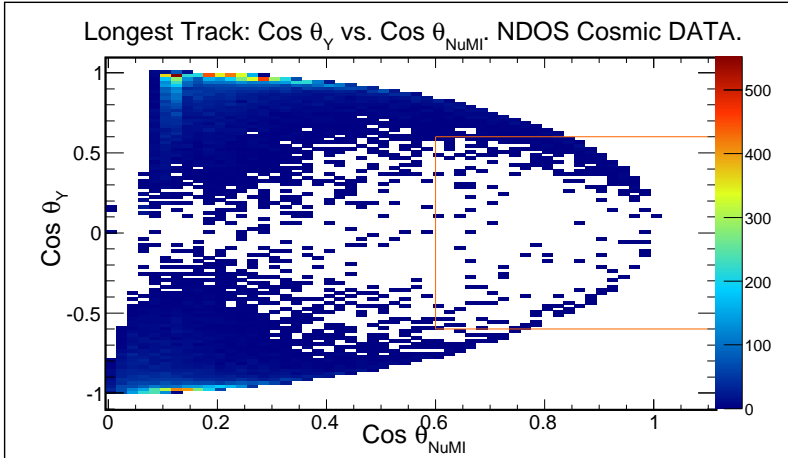

(a)

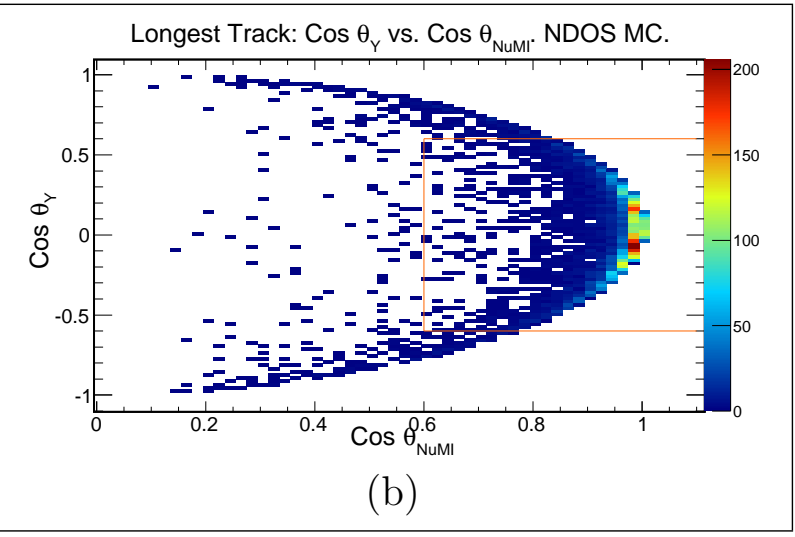

Figure $5.13 \cos \theta_{Y}$ vs. $\cos \theta_{\text {NuMI }}$. (a) Cosmic rays. (b) MC simulation. The orange rectangles define the acceptance region. 
tracks occur in the rejection region defined above, as shown in figure 5.13a, while from figure 5.13b it is clear that the MC signal is mostly inside the acceptance region.

The out-of-time cosmic rays that pass the cuts in relations (5.11) are scaled to the size of the trigger window ${ }^{8}$, and then subtracted from the in-time data that pass these cuts to obtain the final $\nu_{\mu}+\bar{\nu}_{\mu} \mathrm{CC}$ candidates sample. This subtraction is done bin by bin in the number of selected neutrino candidates vs. neutrino energy figure ${ }^{9}$.

\subsubsection{Minimum Ionizing Particle Cut}

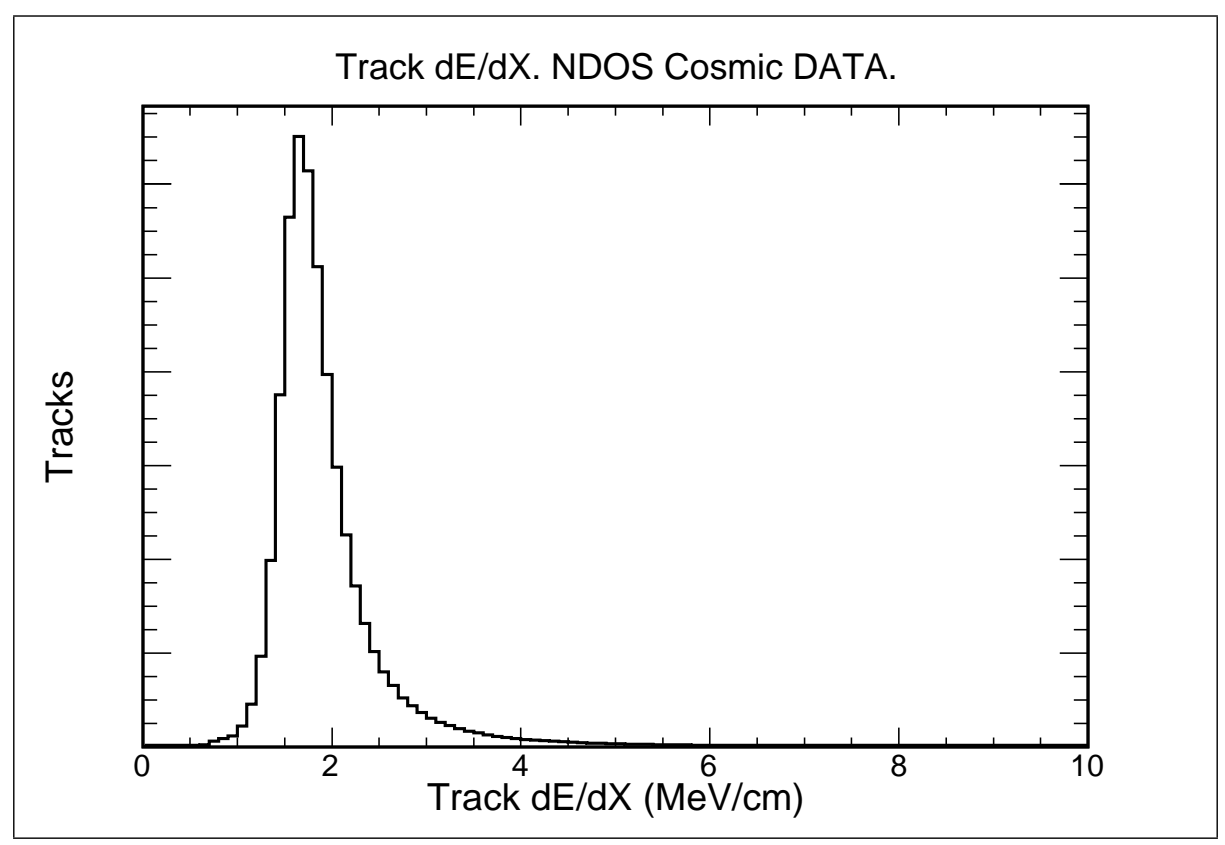

Figure 5.14 Cosmic Track Mean $\boldsymbol{d} \boldsymbol{E} / \boldsymbol{d X}$. NDOS cosmic data.

The longest track in each event is assumed to be a muon. However, a background of other particles, mostly hadrons, is present. The reconstruction algorithms can mistake an electromagnetic shower as a long single track. CC interactions of the $\nu_{e}$ component in the

\footnotetext{
${ }^{8}$ Recall relation (4.11).
}

${ }^{9}$ See figure 8.6. 
NuMI beam can produce an energetic electromagnetic shower. Most of the electromagnetic tracks that fake a muon can be rejected on the basis on their energy deposition profile.

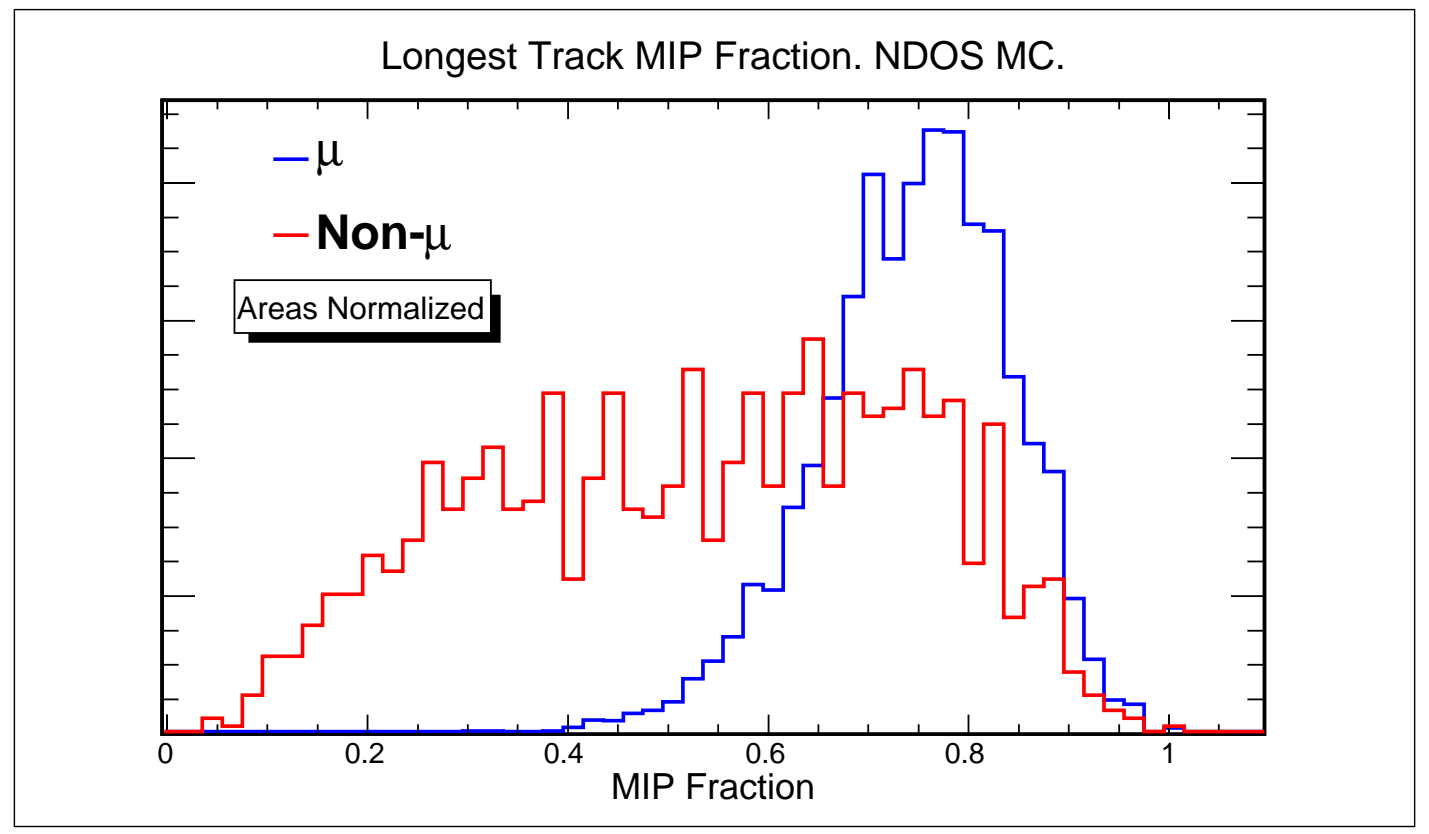

Figure 5.15 MIP Fraction. Muon (blue) and non-muon (red). Areas normalized. MC simulation.

The best way to know the energy deposition profile for muons is to investigate cosmic muons. The distribution of the mean $d E / d X$ of contained cosmic muons is presented in figure 5.14. From this figure it is possible to define a MIP fraction $\left(\mathrm{MIP}_{\text {frac }}\right)$ as the fraction of cells within a track with:

$$
1.1 \mathrm{MeV} / \mathrm{cm} \leq d E / d X \leq 2.7 \mathrm{MeV} / \mathrm{cm}
$$

range that encompasses $90 \%$ of all the cosmic muons, which have a mean of $1.95 \mathrm{MeV} / \mathrm{cm}$. A MIP must have a MIP fraction close to 1 . The MIP fraction for simulated muon and non-muon tracks is shown in figure 5.15. The mean MIP fraction for muons is 0.75 , while that of non-muons is 0.65 . 99.8\% of all muons have MIP fraction higher than 0.4, while 
$69.8 \%$ of all non-muons have MIP fraction higher than 0.4 . These percentages motivate a new cut, the MIP cut, defined as:

$$
\mathrm{MIP}_{\text {frac }}>0.4
$$

The fractions of the distinct particles producing the longest reconstructed track (LRT) are presented in figure 5.16. The fraction of events in which a muon produces the LRT is 0.85 , the other 0.15 is equally shared between protons, charged pions, electrons, and photons ${ }^{10}$. Some of these protons and charged pions come from NC interactions, however most of them are secondary particles from high $y$ (defined in equation (2.17)) interactions in the CC sample. Electrons come from the $\nu_{e} \mathrm{CC}$ background to the $\nu_{\mu} \mathrm{CC}$ signal, and can be rejected based on their energy deposition profile.

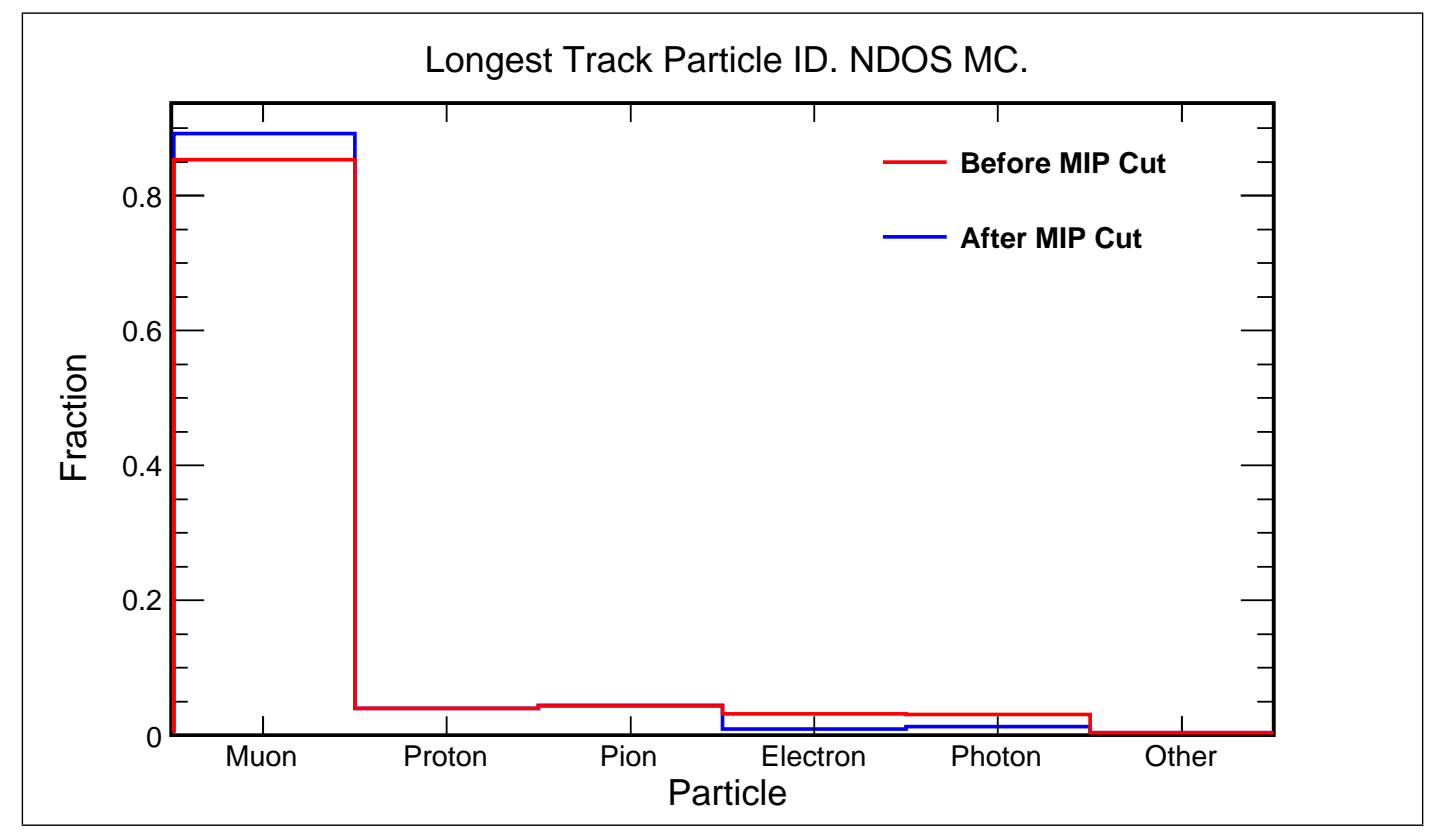

Figure 5.16 Longest Track Particle Identity. Fraction of particles prior (red) and posterior (blue) to the MIP cut. MC simulation.

Rejecting tracks based on relation (5.13) considerably reduces the fraction of electromag-

\footnotetext{
${ }^{10}$ Photons here refer to the primary particle that originates the electromagnetic shower.
} 

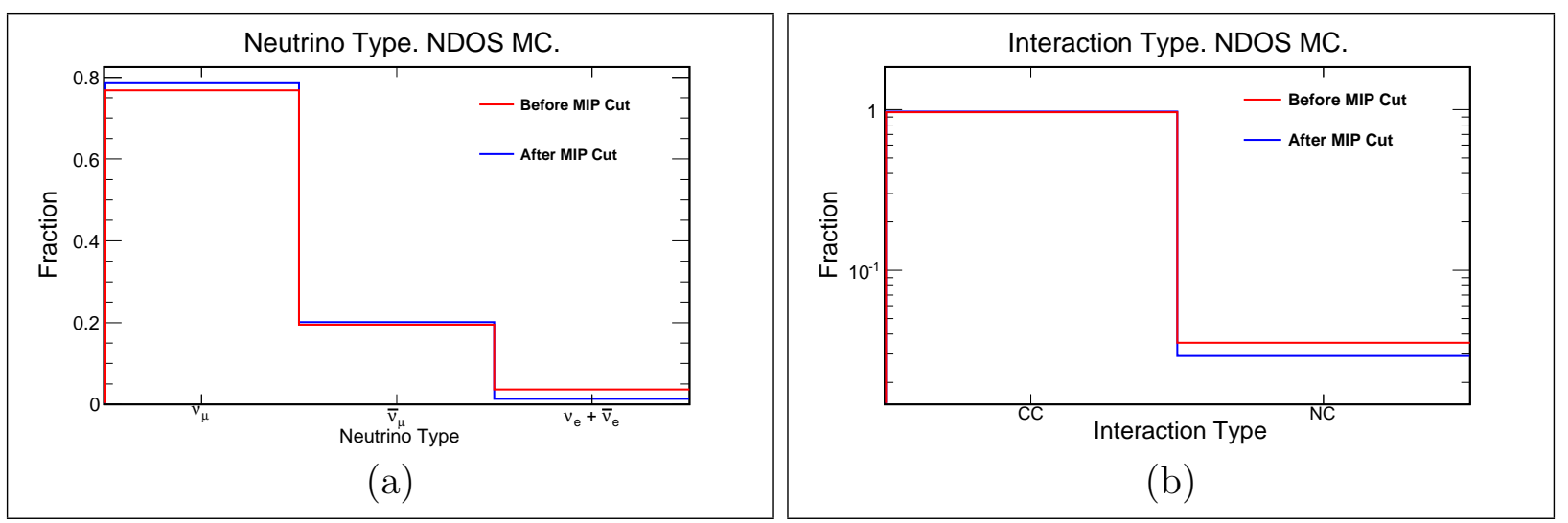

Figure 5.17 Consequences Of The MIP Cut. Before (red) and after (blue) the MIP cut for (a) neutrino types, and (b) interaction types. MC simulation.

netic tracks (from $\nu_{e}+\bar{\nu}_{e} \mathrm{CC}$ ) in the $\nu_{\mu} \mathrm{CC}$ sample from about $4 \%$ to about $1 \%$, as shown in figure 5.16. The impact of this cut on other aspects of the analysis is minimal, as seen from figure 5.17. The composition of the neutrino sample before and after the MIP cut is seen in figure $5.17 \mathrm{a}$, with $1.4 \%$ of $\nu_{e}+\bar{\nu}_{e} \mathrm{CC}$ background. The cut reduces the NC background to a $2.9 \%$ level from the initial $3.5 \%$, as seen in figure $5.17 \mathrm{~b}$. The MC simulation indicates that the purity $(\mathrm{p})$ of the MIP cut is:

$$
\mathrm{p}=\frac{N_{R}}{N_{T}}=(91.3 \pm 0.6) \%
$$

where $N_{R}$ is the number of reconstructed tracks with MIP fraction larger than 0.4 and identified in true as a muon, and $N_{T}$ is the total number of reconstructed tracks with MIP fraction larger than 0.4 . The efficiency $(\epsilon)$ is:

$$
\epsilon=\frac{N_{R}}{N_{e}}=(99.1 \pm 0.2) \%
$$

where $N_{e}$ is the number of true muons. The MIP cut purity and efficiency per energy bins are summarized in table 5.1, at the end of this chapter. 
After the event selection procedure is complete, the sought set of $\nu_{\mu} \mathrm{CC}$ events is obtained. Each of these events goes through a process of energy reconstruction that produces the neutrino energy distribution of the selected sample.

Table 5.1 shows that the efficiency on the track length cut (TLC) increases with neutrino energy as the LTL reconstruction efficiency increases with length. The purity on the containment cut (CR) decreases with increasing neutrino energy as the muons become more energetic and leave the detector more frequently. Table 5.1 also shows how the MIP cut purity decreases with increasing neutrino energy as the contained reconstructed longest track is not a muon. This is because the energetic muon escapes the detector more frequently for high y CC interactions. All the efficiencies and purities shown in table 5.1 are calculated to know the performance of each particular selection criterion. The calculations done for a particular selection criterion have no dependency on previous or posterior selection criteria. 


\begin{tabular}{|c|c|c|c|c|c|c|c|c|}
\hline Cut $\backslash \mathrm{E}(\mathrm{GeV})$ & $0.5-1.0$ & $1.0-1.5$ & $1.5-2.0$ & $2.0-2.5$ & $2.5-3.0$ & $3.0-3.5$ & $3.5-4.0$ & $4.0-10.0$ \\
\hline \hline 3D & & & & & & & & \\
Efficiency & $74.8 \%$ & $79.9 \%$ & $80.2 \%$ & $80.6 \%$ & $81.3 \%$ & $81.4 \%$ & $80.3 \%$ & $80.7 \%$ \\
Error & $0.3 \%$ & $0.3 \%$ & $0.2 \%$ & $0.3 \%$ & $0.5 \%$ & $0.7 \%$ & $0.9 \%$ & $0.5 \%$ \\
\hline TLC & & & & & & & & \\
Purity & $95.7 \%$ & $94.6 \%$ & $95.1 \%$ & $94.8 \%$ & $93.4 \%$ & $92.4 \%$ & $90.7 \%$ & $92.2 \%$ \\
Error & $0.2 \%$ & $0.2 \%$ & $0.1 \%$ & $0.2 \%$ & $0.4 \%$ & $0.5 \%$ & $0.7 \%$ & $0.4 \%$ \\
Efficiency & $38.8 \%$ & $53.8 \%$ & $59.3 \%$ & $63.2 \%$ & $69.2 \%$ & $71.5 \%$ & $76.0 \%$ & $81.6 \%$ \\
Error & $0.4 \%$ & $0.4 \%$ & $0.3 \%$ & $0.4 \%$ & $0.7 \%$ & $0.9 \%$ & $1.0 \%$ & $0.6 \%$ \\
\hline VR & & & & & & & & \\
Purity & $97.4 \%$ & $97.0 \%$ & $96.6 \%$ & $96.6 \%$ & $96.4 \%$ & $95.4 \%$ & $94.2 \%$ & $95.3 \%$ \\
Error & $0.3 \%$ & $0.2 \%$ & $0.2 \%$ & $0.2 \%$ & $0.4 \%$ & $0.5 \%$ & $0.7 \%$ & $0.4 \%$ \\
Efficiency & $97.0 \%$ & $96.4 \%$ & $95.6 \%$ & $95.5 \%$ & $94.9 \%$ & $94.8 \%$ & $93.0 \%$ & $93.9 \%$ \\
Error & $0.4 \%$ & $0.3 \%$ & $0.2 \%$ & $0.2 \%$ & $0.5 \%$ & $0.6 \%$ & $0.8 \%$ & $0.4 \%$ \\
\hline CR & & & & & & & & \\
Purity & $97.7 \%$ & $94.4 \%$ & $88.2 \%$ & $83.6 \%$ & $86.4 \%$ & $80.0 \%$ & $71.6 \%$ & $55.6 \%$ \\
Error & $0.4 \%$ & $0.4 \%$ & $0.4 \%$ & $0.5 \%$ & $0.8 \%$ & $1.2 \%$ & $1.6 \%$ & $1.1 \%$ \\
Efficiency & $71.4 \%$ & $68.8 \%$ & $72.1 \%$ & $73.8 \%$ & $84.4 \%$ & $86.2 \%$ & $80.6 \%$ & $73.2 \%$ \\
Error & $1.1 \%$ & $0.7 \%$ & $0.5 \%$ & $0.5 \%$ & $0.9 \%$ & $1.0 \%$ & $1.4 \%$ & $1.0 \%$ \\
\hline MIP & & & & & & & & \\
Purity & $99.1 \%$ & $94.3 \%$ & $90.0 \%$ & $87.7 \%$ & $87.2 \%$ & $83.3 \%$ & $77.7 \%$ & $58.6 \%$ \\
Error & $0.3 \%$ & $0.5 \%$ & $0.6 \%$ & $0.7 \%$ & $1.4 \%$ & $1.8 \%$ & $2.6 \%$ & $2.4 \%$ \\
Efficiency & $99.9 \%$ & $99.8 \%$ & $99.9 \%$ & $99.9 \%$ & $99.6 \%$ & $100 \%$ & $99.4 \%$ & $98.4 \%$ \\
Error & $0.1 \%$ & $0.1 \%$ & $0.1 \%$ & $0.1 \%$ & $0.2 \%$ & 0 & $0.5 \%$ & $0.6 \%$ \\
\hline
\end{tabular}

Table 5.1 Event Selection Performance. Purity and efficiency for the various cuts made during the event selection. The columns in this table represent the energy bins used in this analysis. The last column includes energies from $4.0 \mathrm{GeV}$ to $10 \mathrm{GeV}$. No statistically significant data exist below $0.5 \mathrm{GeV}$. 


\section{Chapter 6}

\section{Event Energy Reconstruction}

The energy of a neutrino interaction must be determined from the energy deposited in the detector by the final state particles. In $\nu_{\mu} \mathrm{CC}$ events, different physical processes are involved in the determination of the energy of the muon or the hadronic system. In some cases, a muon will stop in the detector before it decays or is captured in an atomic orbit. The ionization energy loss mechanism of a muon is well understood, so that the energy of a muon can be directly related to its range, which is estimated by its track length in the detector ${ }^{1}$. The energy deposited in the detector by hadrons is reconstructed using the detector as a calorimeter, i.e. the deposited energy is scaled, using the hadronic energy in the MC simulation, in order to estimate the full energy encompassed within the hadronic system.

\subsection{Muon Energy Estimation}

The MC simulation of the particles interacting with the detector incorporates the ionization properties of the muon as well as the distortions introduced by the detector structure and operation. For muon energies between $0.4 \mathrm{GeV}$ to above $3 \mathrm{GeV}$, and the mixture of materials of the $\mathrm{NO} \nu \mathrm{A}$ detectors (average detector density of $1.02 \mathrm{~g} / \mathrm{cm}^{3}[107]$ ), the corresponding ionization energy loss rates vary from $2.1 \mathrm{MeV} \mathrm{cm} / \mathrm{g}$ to $2.3 \mathrm{MeV} \mathrm{cm} / \mathrm{g}$. As the muon

\footnotetext{
${ }^{1}$ See [87] for a detailed discussion on passage of particles through matter.
} 


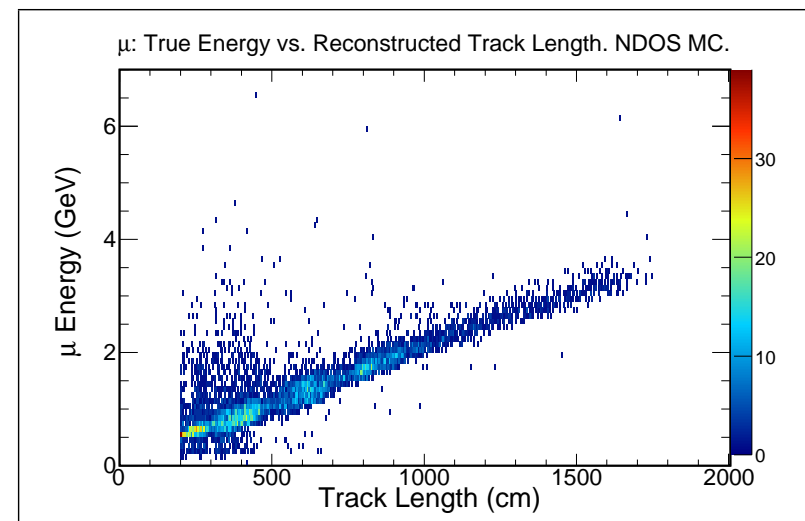

(a)

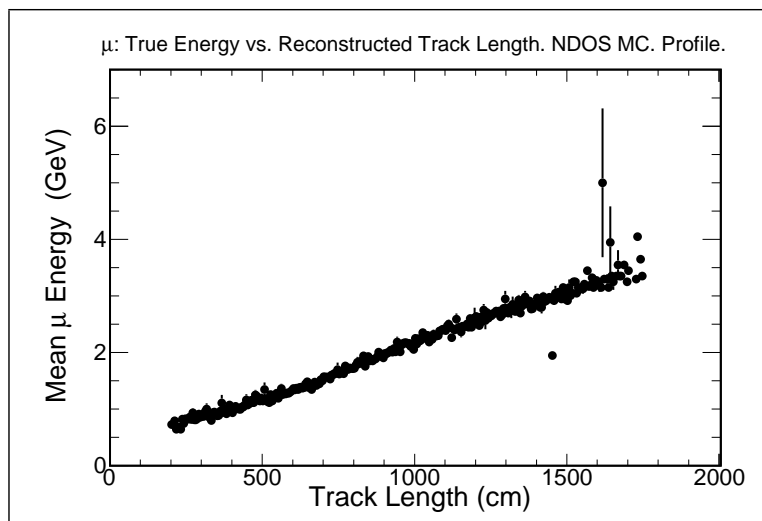

(b)

Figure 6.1 Muon: True Energy vs. Reconstructed Track Length. (a) True energy of muons as a function of the reconstructed track length. (b) Profile plot of (a). MC simulation.

energy drops below $0.4 \mathrm{GeV}$, the energy loss rate rises and reaches higher ionization levels shortly before stopping. Therefore, the experimentally determined muon energy and the range are nearly linearly related. The true muon energy and its reconstructed track length are strongly correlated, as shown in figure 6.1. The reconstructed track length is used to determine the energy of each observed muon. The true energy of the muons $\left(E_{T}\right)$ as a function of their reconstructed track length $\left(L_{R}\right)$ is (refer to figure 6.1a):

$$
E_{T}=p_{0}+p_{1} L_{R},
$$

where the $p_{i}$ are parameters to be determined. Deviations from equation (6.1), particularly at short track lengths, come from uncontained muons, as described in section 4.3.2. Equation (6.1) is a good approximation to describe the relationship between the true energy and the reconstructed track length (longer than $200 \mathrm{~cm}$ ) of the muons; however, the data shown in figure 6.1a do not follow a straight line, these are scattered inside a narrow band with some width. In order to reconstruct the energy of the muon, the most probable value of true energy per reconstructed track length bin is calculated and drawn as a function of reconstructed 
track length, as shown in figure 6.1b. The error bars are the spread of the energy per track length bin.

The $p_{i}$ in equation (6.1) are functions of $L_{R}$, and are set equal to parameters obtained from straight line fits. More than one straight line fit are required to better represent different regions in figure 6.1b. The region: $L_{R}<580 \mathrm{~cm}$ (refer to figure $6.2 \mathrm{a}$ ), has $\chi^{2}=90.91$ for ndf $=74$. The region: $580 \mathrm{~cm}<L_{R}<920 \mathrm{~cm}$ (refer to figure 6.2b), has $\chi^{2}=72.34$ for ndf $=66$. The last region: $L_{R}>920 \mathrm{~cm}$ (refer to figure $6.2 \mathrm{c}$ ), has $\chi^{2}=2634.5$ for ndf $=144$, and is more scattered than the first one. The three regions combined have $\chi^{2}=9340.46$ for ndf $=284$. In order to minimize the $\chi^{2}$, a handful of straight line fits are calculated for the first and last regions. For the last region, e.g., there are five fits with the following results:

- $920 \mathrm{~cm}<L_{R}<1115 \mathrm{~cm}: \chi^{2}=56.6$ for $\mathrm{ndf}=37$.

- $1115 \mathrm{~cm}<L_{R}<1285 \mathrm{~cm}: \chi^{2}=53.4$ for $\mathrm{ndf}=32$.

- $1285 \mathrm{~cm}<L_{R}<1375 \mathrm{~cm}: \chi^{2}=30.2$ for $\mathrm{ndf}=16$.

- $1375 \mathrm{~cm}<L_{R}<1490 \mathrm{~cm}: \chi^{2}=30.6$ for $\mathrm{ndf}=20$.

- $1490 \mathrm{~cm}<L_{R}: \chi^{2}=132.3$ for ndf $=31$.

All the straight line fits are constrained to be continuous. The parameters of the fits are used to obtain a first order approximation of the reconstructed energy.

The final version of the reconstructed muon energy is obtained after taking the difference of the true energy and the approximated reconstructed energy. Small corrections, $C_{b}$, of the order of $1 \%$ of the muon energy, are added to these approximated energies, per track length bin, in order to achieve that the mean of the difference between the true energy $\left(E_{T}\right)$ and the reconstructed energy $\left(E_{R}\right)$ of the muon is equal to zero: $\Delta E=E_{T}-E_{R}=0$, as seen in 
figure 6.3a. Finally, the reconstructed energy of the muon is:

$$
E_{R}=p_{0}\left(L_{R}\right)+p_{1}\left(L_{R}\right) L_{R}+C_{b}\left(L_{R}\right) .
$$

The parameters of the fits and corrections are used to obtain the reconstructed muon energy in data.
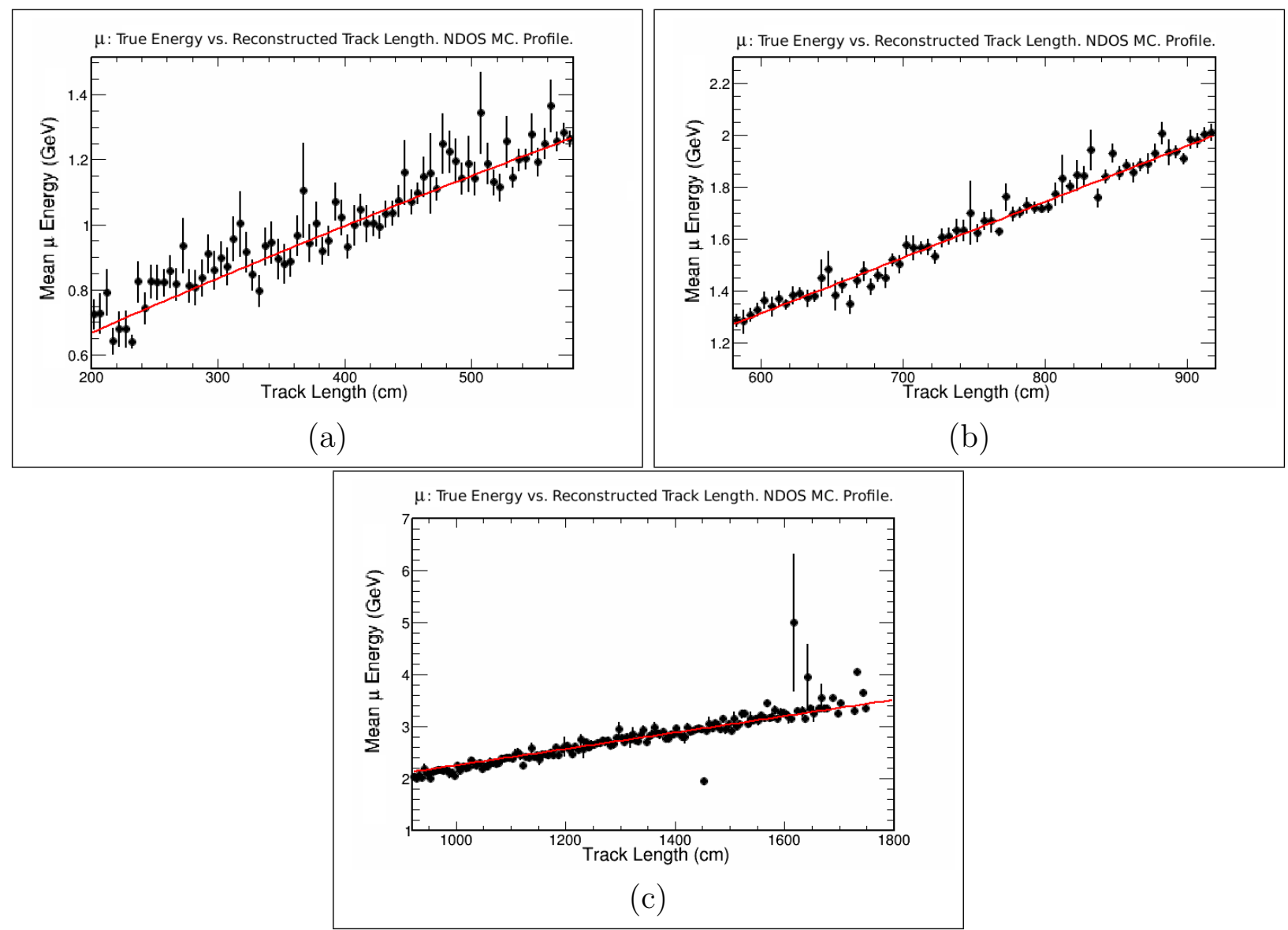

Figure 6.2 Profile Plots Of Figure 6.1a. (a) $L_{R}<580 \mathrm{~cm}$. (b) $580 \mathrm{~cm}<L_{R}<920 \mathrm{~cm}$. (c) $L_{R}>920 \mathrm{~cm}$.

The uniformity of the density of the NDOS is broken at the muon catcher, thus, muons that reach the muon catcher require an additional term in equation (6.2) that would take into account the portion of the muon's trajectory that goes through the steel. For these 
muons, equation (6.2) changes to:

$$
E_{R}=p_{0}\left(L_{R}\right)+p_{1}\left(L_{s}\right) L_{s}+p_{2}\left(L_{\mu c}\right) L_{\mu c}+C_{b}\left(L_{R}\right)
$$

where $L_{s}$ is the portion of the muon's trajectory that goes through the plastic + scintillator bulk, the $p_{2}$ are fit parameters, and $L_{\mu c}$ is the portion of the trajectory that goes through the muon catcher. The reconstructed track length is: $L_{R}=L_{s}+L_{\mu c}$.
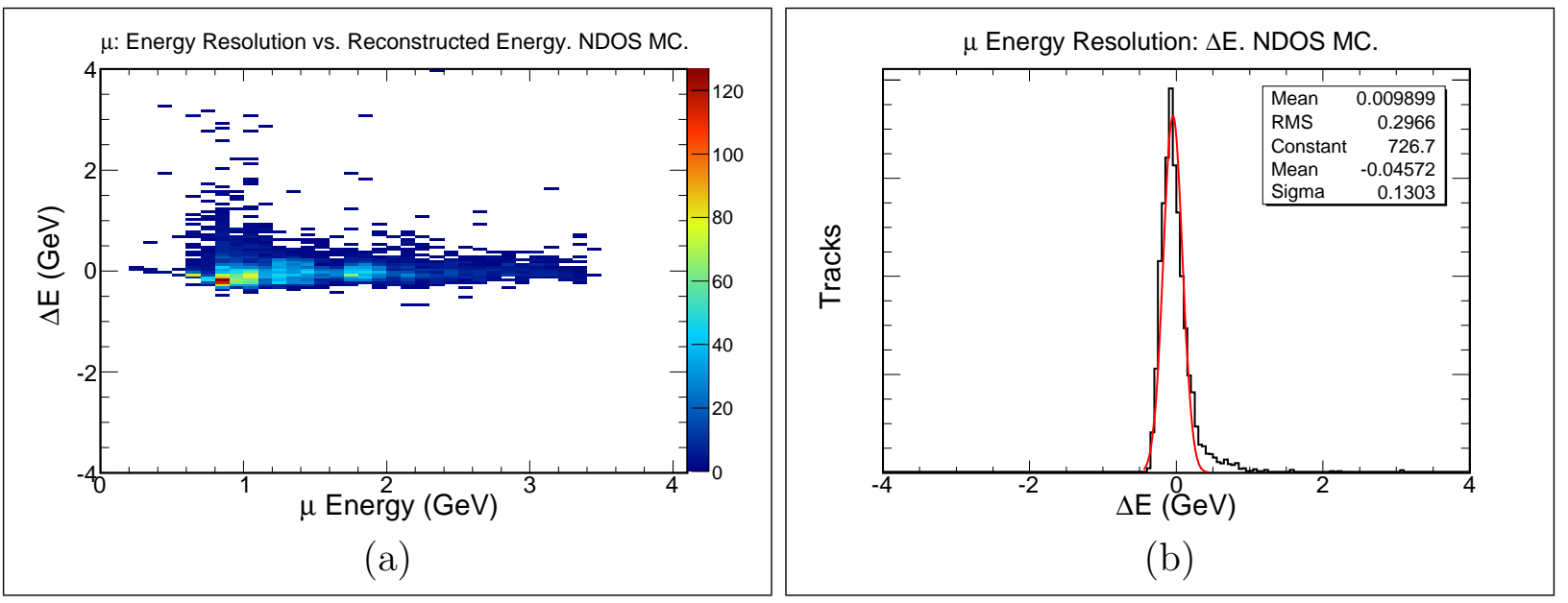

Figure 6.3 Muon Energy Resolution. (a) True minus reconstructed muon energy $(\Delta E)$ as a function of the reconstructed muon energy. The average $\Delta E$ is effectively zero. (b) Overall muon energy resolution. MC simulation.

The overall muon reconstructed energy resolution is obtained from the Gaussian fit shown in figure 6.3b. The spread in the $\Delta E$ from the fit is the reconstructed energy resolution. The tail of the distribution accounts for about $5 \%$ of the muons. These are considered contained, but their true trajectory leaves the detector, therefore the difference between true and reconstructed energies is positive and not close to zero. From the spread in the fit, the resolution in reconstructed muon energy is $130 \mathrm{MeV}$. The muon energy resolution at energies higher than $2 \mathrm{GeV}$ is better than lower energies since the containment issues are less frequent when compared to shorter, and less energetic, muon tracks. High energy muons 


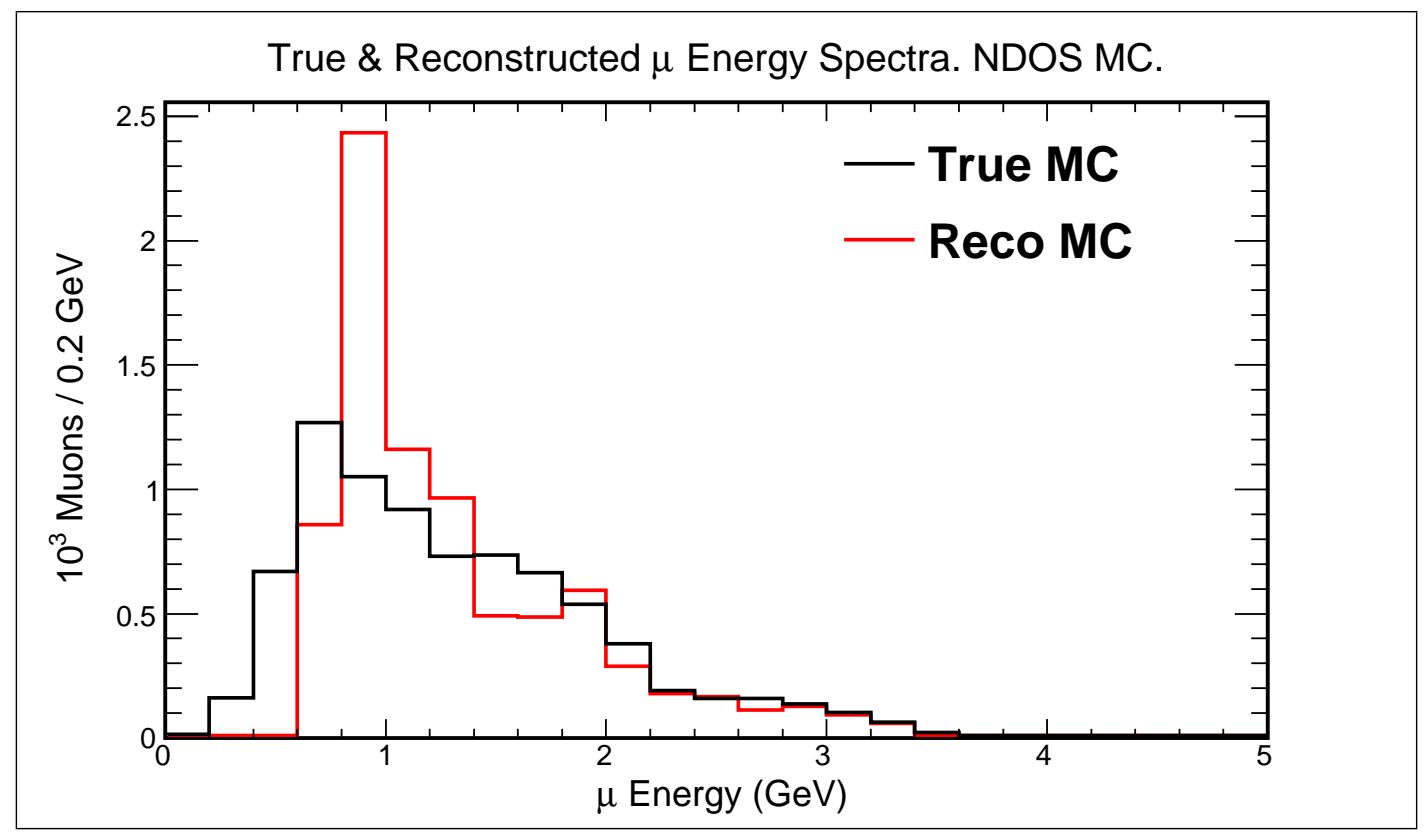

Figure 6.4 Muon Energy Distributions. Muon true (black), and reconstructed (red) energy distributions. MC simulation.

often have their momentum vector almost parallel to the beam's direction, and these reach the muon catcher, which helps to contain them. Around $1 \mathrm{GeV}$ the resolution is about $14 \%$, i.e. $140 \mathrm{MeV}$.

Even with the best attempts to properly reconstruct the muon energy, a comparison of the simulated energy for muons in CC events with their reconstructed values shows that there is a tendency to move muons with energies lower than $0.6 \mathrm{GeV}$ to an energy above $0.6 \mathrm{GeV}$, as shown in figure 6.4. The reconstructed muons with energies about $1 \mathrm{GeV}$ sharply peak while the true muon energy is more spread out. This is the result of the LTL corrections described in section 4.3.2. 


\subsection{Hadronic Energy Estimation}

The $\mathrm{NO} \nu \mathrm{A}$ detectors were designed to obtain excellent electromagnetic shower identification and energy resolution. This is accomplished with a detector comprised of primarily carbon and hydrogen (low $Z$ nuclei), resulting in an electromagnetic sampling fraction better than $65 \%$ [107]. These characteristics of the detector, however, result in a poor performance as a hadronic calorimeter.

CC interactions produce hadrons in addition to the characteristic lepton. Hadronic energy estimation is different from electromagnetic energy estimation (see [87] for details). Recalling section 4.3.2, protons and charged pions often do not leave hit patterns to be reconstructed into tracks in the $\mathrm{NO} \nu \mathrm{A}$ detector, resulting in degraded energy resolution [108]. The presence of hadronic showers combined with the neutrino event containment details studied in section 4.3.2, have as a consequence that the hadronic energy estimation is done by converting deposited energy in the detector to real deposited energy.

The observed hadronic energy is defined as the sum of all the deposited energy in the event that does not belong to the longest track, which is assumed to be the muon. Different zones in the NDOS VR measure deposited energy differently depending on their location and number of active channels. Since the neutrino event containment is only determined by the longest track properties, it is necessary to introduce a method to take into account hadronic energy that leaves the detector, is deposited in dead material or in cells without APDs. Therefore, the energy corrections are determined by dividing the NDOS VR into 6 zones, as shown in figure 6.5. The limits of these zones are defined by:

- Zone 1: $53 \mathrm{~cm}>|\mathrm{X}|>106 \mathrm{~cm}, 288 \mathrm{~cm}<\mathrm{z}<345 \mathrm{~cm}$.

- Zone 2: $53 \mathrm{~cm}>|\mathrm{X}|>106 \mathrm{~cm}, 345 \mathrm{~cm}<\mathrm{z}<402 \mathrm{~cm}$. 


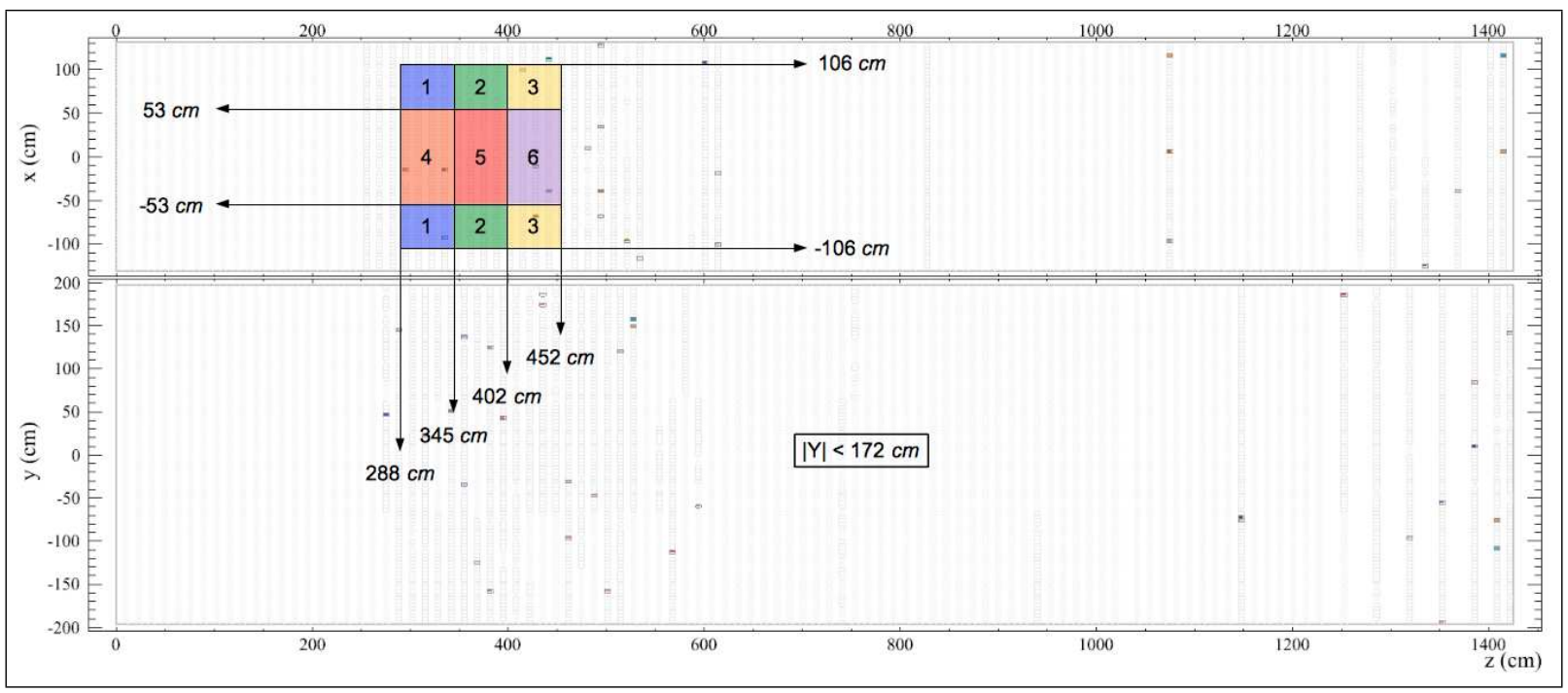

Figure 6.5 Hadronic Energy Classification Zones. The coordinates of the edges of the distinct zones are shown with arrows. All zones fulfill the condition: $172 \mathrm{~cm}>|\mathrm{Y}|$. Zone 1 is the union of the two zones labeled with the number: 1. These two zones are equivalent, and therefore are united into one. The same is true for the zones 2 and 3. Zones 4 through 6 are in the middle of the detector.

- Zone 3: $53 \mathrm{~cm}>|\mathrm{X}|>106 \mathrm{~cm}, 402 \mathrm{~cm}<\mathrm{z}<452 \mathrm{~cm}$.

- Zone 4: $53 \mathrm{~cm}<|\mathrm{X}|, 288 \mathrm{~cm}<\mathrm{z}<345 \mathrm{~cm}$.

- Zone 5: $53 \mathrm{~cm}<|\mathrm{X}|, 345 \mathrm{~cm}<\mathrm{z}<402 \mathrm{~cm}$.

- Zone 6: $53 \mathrm{~cm}<|\mathrm{X}|, 402 \mathrm{~cm}<\mathrm{z}<452 \mathrm{~cm}$.

All 6 zones are rectangular boxes fulfilling the condition: $172 \mathrm{~cm}>|\mathrm{Y}|$. Events are classified by the zone in which their vertex is located. Events with an interaction occurring in zones 1 through 3 will have a higher probability for energy to leave through the sides of the detector, when compared to the other zones. In addition, zone 3 will have a higher probability to leak energy into the region without active cells, when compared to the other zones. Events with its neutrino interaction occurring in zone 6 will deposit a good fraction of their energy into the region without active cells. Events with its neutrino interaction 
occurring in zones 4 and 5 will deposit most of the hadronic energy inside the detector and the NDOS VR. All these zones have inactive or missing channels. Due to the differences in energy deposition mentioned above, each zone needs its own energy estimation parameters. Energy estimation parameters for each zone are determined through a comparison between the deposited hadronic energy (the energy read by detector) and the true energy of the hadronic system.

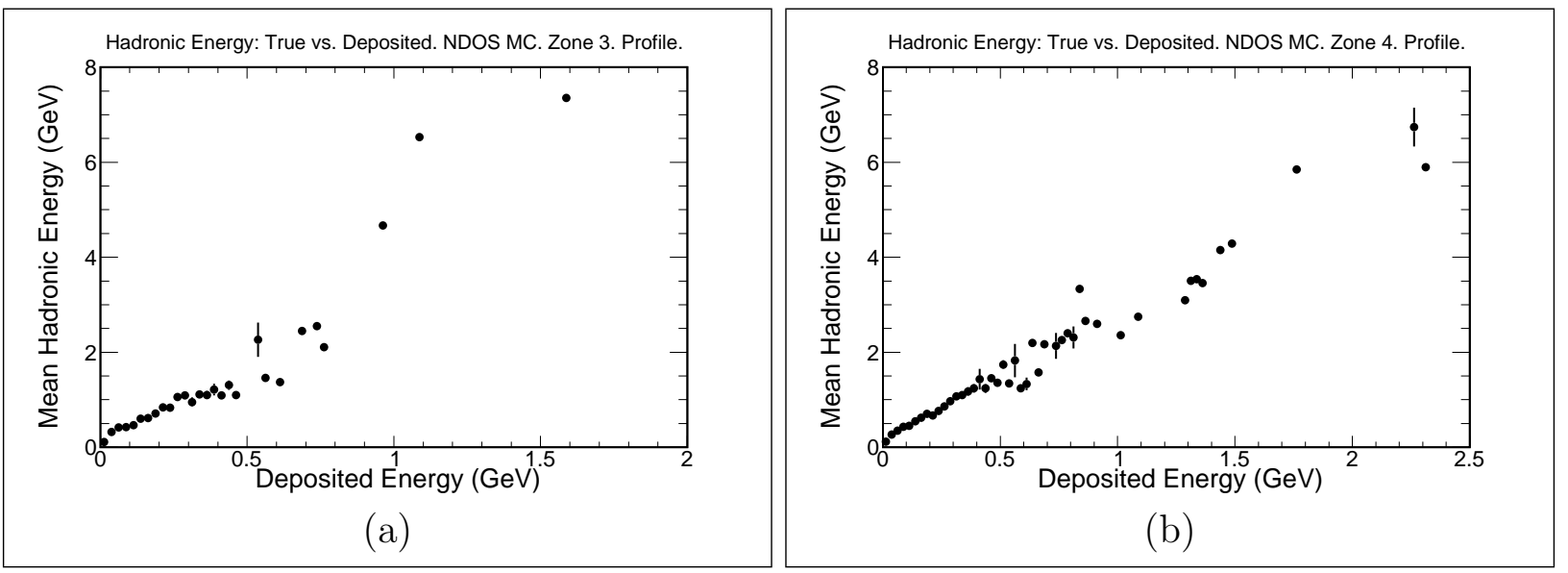

Figure 6.6 True Hadronic Energy vs. Deposited Energy. Mean true hadronic energy per deposited energy bin as a function of the deposited hadronic energy of (a) zone 3, and (b) zone 4. MC simulation.

The mean true hadronic energy, per deposited hadronic energy bin, as a function of the deposited hadronic energy of zone 3 is shown in figure $6.6 \mathrm{a}$. Zone 3 is the sample of events that deposits less energy into the detector, when compared to the other zones. Figure 6.6b is the equivalent of figure $6.6 \mathrm{a}$ for zone 4 , which is the zone that gets the largest hadronic energy deposition of all 6 zones. The correlations between true hadronic energy $\left(H_{T}\right)$ and deposited hadronic energy $\left(H_{R}\right)$, which are distinctly non-linear in all the 6 zones, help to define the reconstructed hadronic energy $\left(E_{\text {had }}\right)$. These correlations are presented by polynomials. The degree $(A)$ of the polynomial is a function of the zone. The coefficients of 
the polynomials $\left(q_{a}\right)$ are used to obtain the $E_{\text {had }}$ :

$$
E_{\mathrm{had}}=\sum_{a=0}^{A} q_{a} H_{R}^{a}
$$

There is one equation (6.4) per zone.
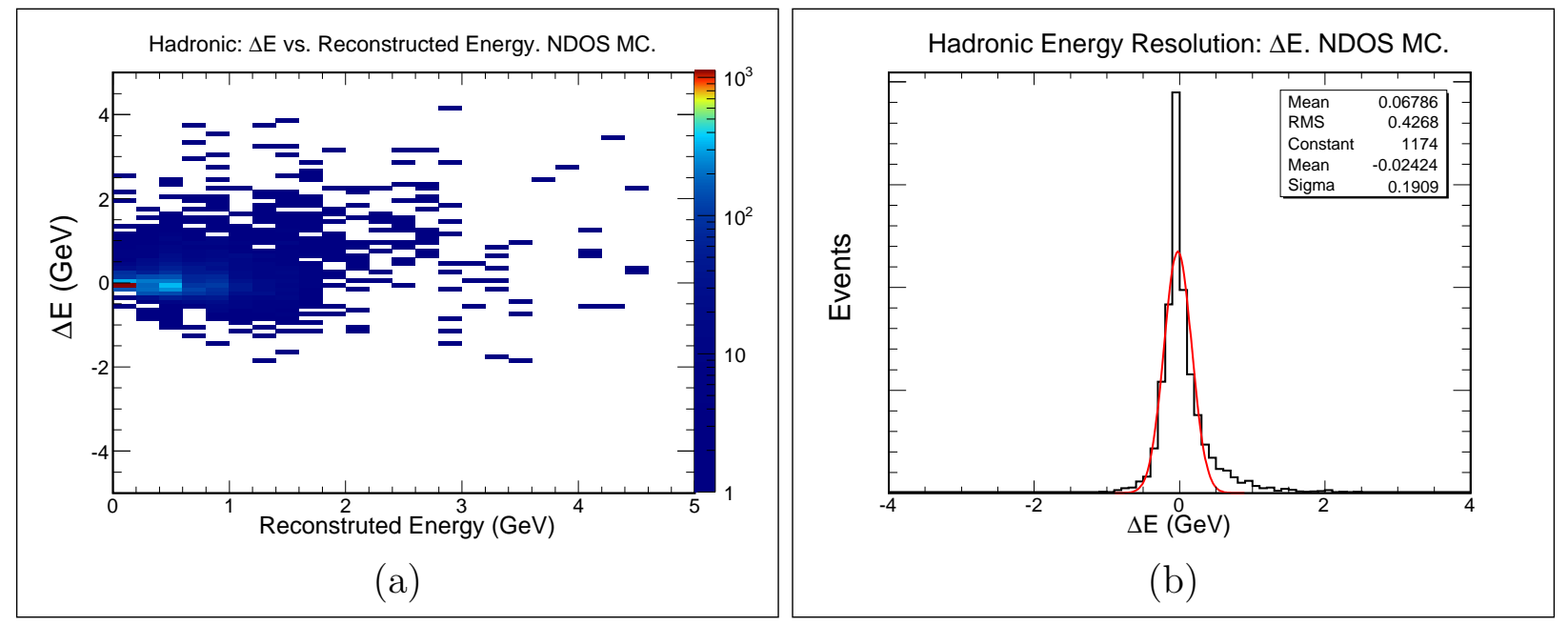

Figure 6.7 Hadronic Energy Resolution. (a) True minus reconstructed hadronic energy $(\Delta E)$ as a function of the reconstructed hadronic energy. The average $\Delta E$ is effectively zero. (b) Overall hadronic energy resolution. MC simulation.

Similar to the muon case, each equation (6.4) requires small corrections, $\mathcal{C}_{g}$, of the order of $5 \%$ of the hadronic energy, per deposited hadronic energy bin, in order to achieve that the mean of the difference of energies $\left(\Delta E=H_{T}-E_{\text {had }}=0\right)$ in the hadronic system is equal to zero, as seen in figure 6.7 a. Finally, the hadronic reconstructed energy, $E_{\text {had }}$, is:

$$
E_{\text {had }}=\sum_{a=0}^{A} q_{a} H_{R}^{a}+C_{g}\left(H_{R}\right) .
$$

The hadronic energy resolution, in figure $6.7 \mathrm{a}$, at $1 \mathrm{GeV}$ is $330 \mathrm{MeV}(33 \%$ ), at $180 \mathrm{MeV}$ is $0.5 \mathrm{GeV}(36.5 \%)$, and it is $70 \mathrm{MeV}$ at $0.2 \mathrm{GeV}(35 \%)$. From the Gaussian fit shown in figure $6.7 \mathrm{~b}$, the overall hadronic energy resolution is $190 \mathrm{MeV}$. The tails account for about $5 \%$ of 


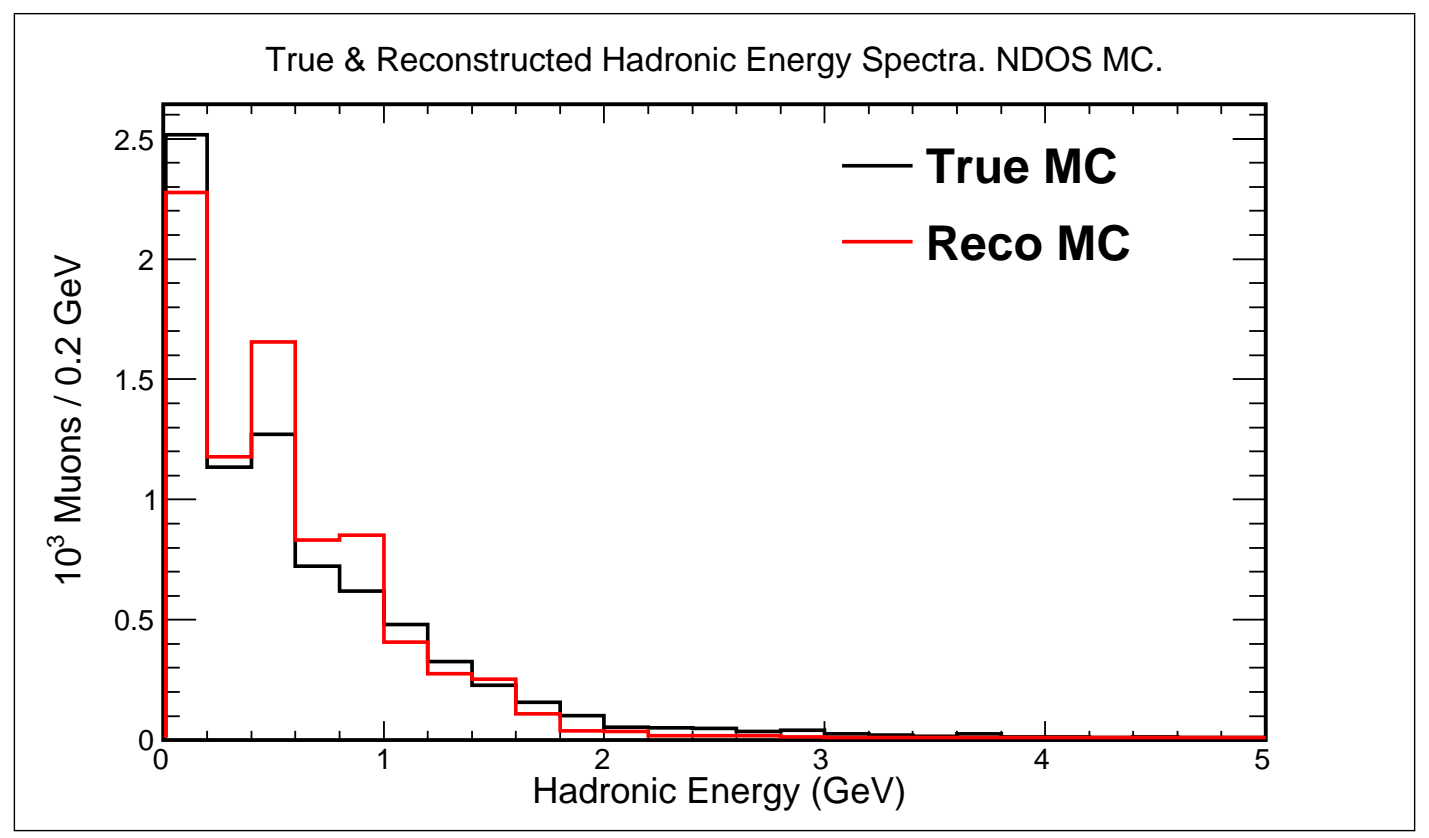

Figure 6.8 Hadronic Energy Distributions. True hadronic (black) and reconstructed (red) energy distributions. MC simulation.

all events.

The distributions in reconstructed and true hadronic energies shown in figure 6.8 have bins of $200 \mathrm{MeV}$. From figure 6.6 it is clear that the spread of the data at energies above $1 \mathrm{GeV}$ introduces deviations from the fits, and therefore underestimation of the hadronic energies larger than $1.6 \mathrm{GeV}$ is seen in figure 6.8 .

\subsection{Quasi-elastic And Non-quasi-elastic Classification}

Section 2.1 presented differences between QE and non-QE interactions. Besides those differences, the two samples also present different efficiencies in the reconstruction process. These two different neutrino interaction samples are used separately to calculate e.g. neutrino fluxes and cross sections. Among the differences presented in section 2.1 is the hadronic energy deposition, which is in general higher for non-QE interactions. This difference in 


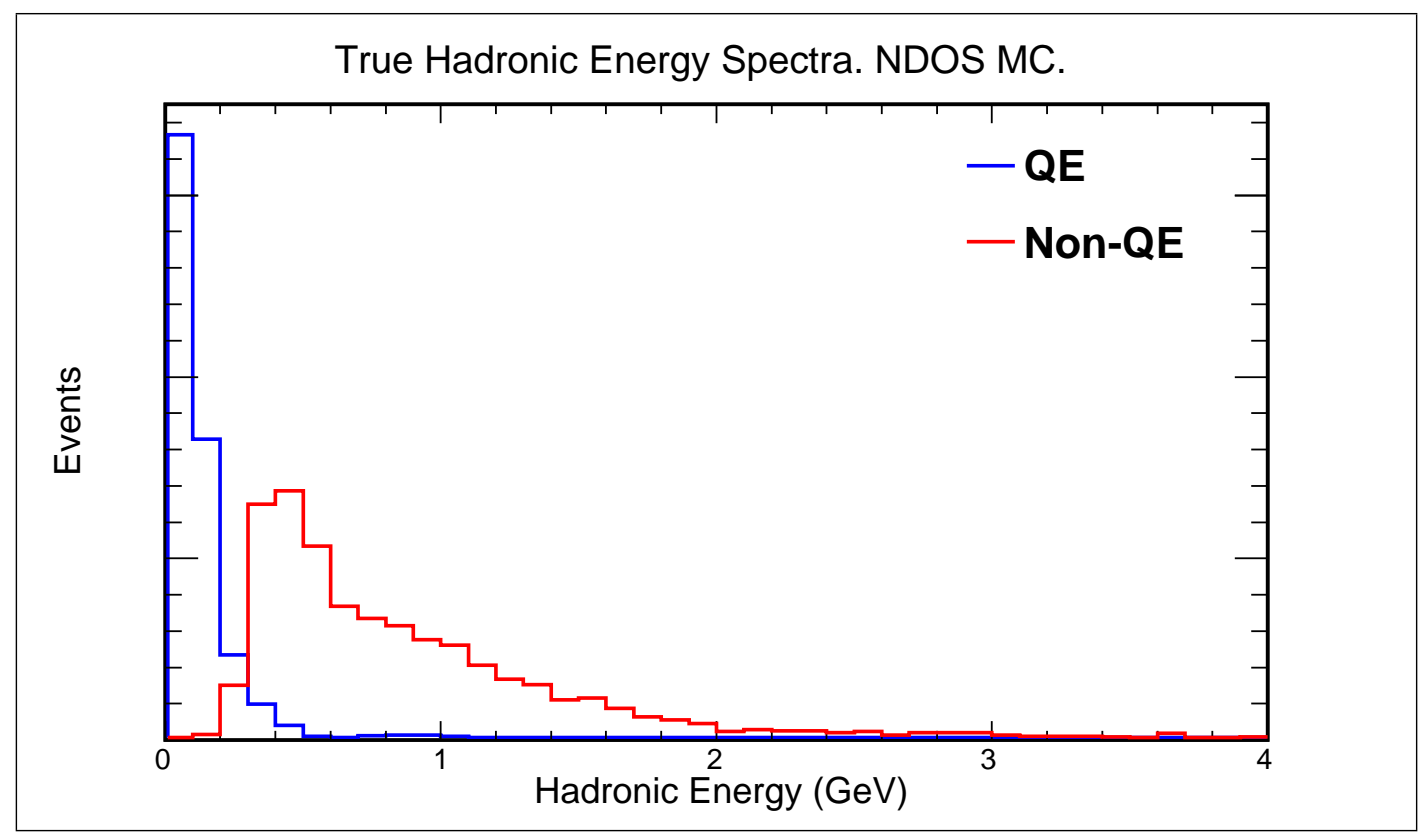

Figure 6.9 QE And Non-QE Hadronic Energy Distributions. True hadronic QE (blue) and non-QE (red) energy distributions. MC simulation.

hadronic energy deposition is presented in figure 6.9. The mean true hadronic energy for the QE sample is $150 \mathrm{MeV}$, while the mean for the non-QE sample is $910 \mathrm{MeV}$. The QE true hadronic energy falls rapidly after $200 \mathrm{MeV}$, and it is at this same energy that the nonQE true hadronic energy starts to become important. Therefore, a separation is possible following the criteria:

$$
E_{\text {had }}<200 \mathrm{MeV} \rightarrow \text { QE, } E_{\text {had }}>200 \mathrm{MeV} \rightarrow \text { non-QE }
$$

With this separation, $84.1 \%$ of all true QE events, and $0.4 \%$ of all true non-QE events have true hadronic energy lower than $200 \mathrm{MeV}$. Relations (6.6) are applied to the reconstructed hadronic energy to make the separation between the QE and the non-QE samples.

The comparisons between true and reconstructed muon and hadronic energy are presented in figure 6.10. In these figures the true $\mathrm{MC}$ distributions represent the $\mathrm{QE}$ and 

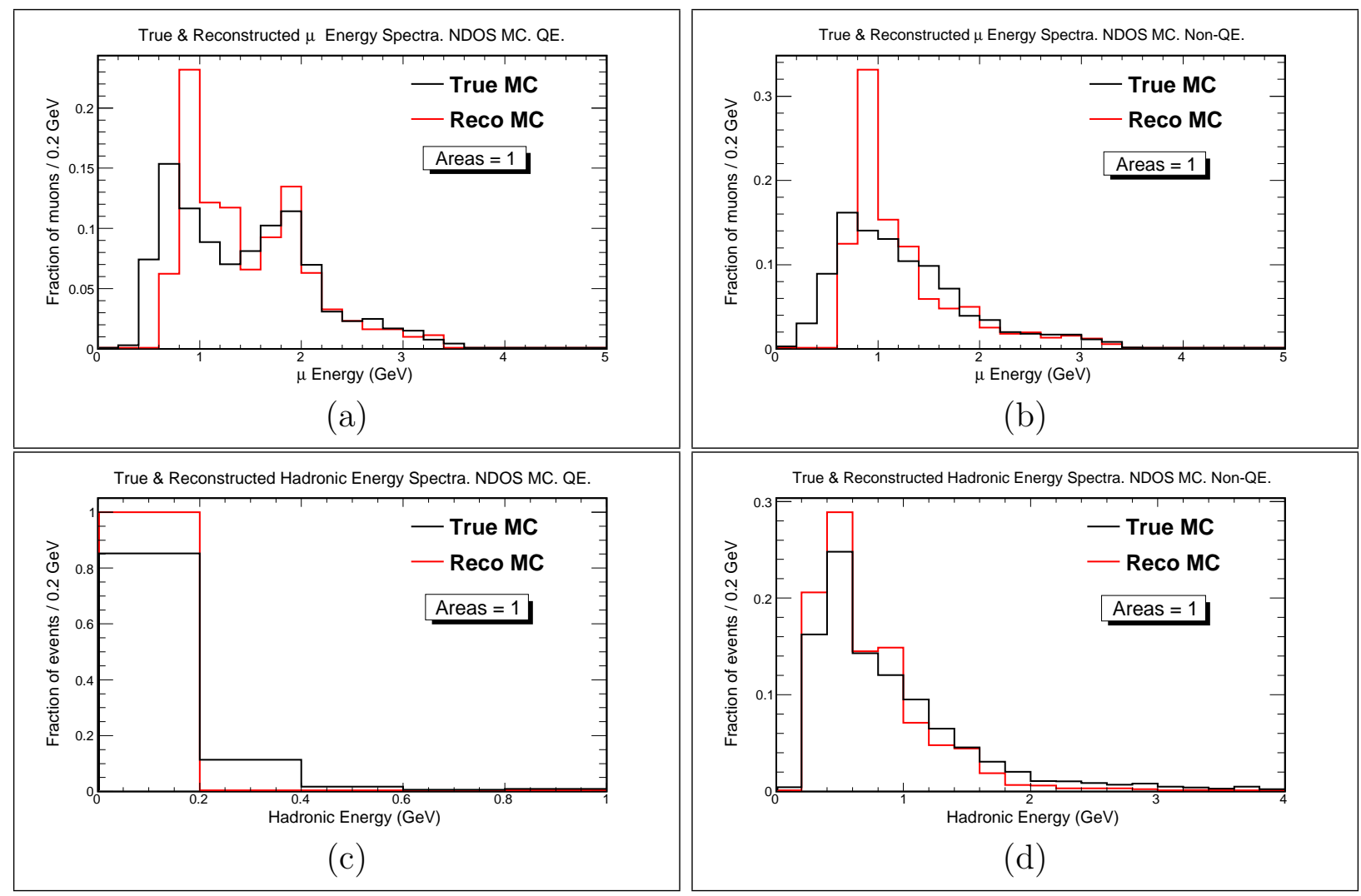

Figure 6.10 Muon And Hadronic Energy Distributions For The QE And Non-QE Events. The energy distributions for the true (black) and reconstructed (red) samples, with muon energy in (a) QE and (b) non-QE events, and hadronic energy in the (c) QE and (d) non-QE events. Areas normalized to 1 in order to compare the shapes of the distributions. MC simulation. 
non-QE interactions as defined in the MC simulation. The reconstructed MC distributions represent the interactions as defined by relations (6.6). The poor tracking coverage for muons immediately downstream of the VR causes muon energies below $0.6 \mathrm{GeV}$ to be systematically reconstructed to energies higher than the true values by $0.2 \mathrm{GeV}$ (in the QE case), and $0.4 \mathrm{GeV}$ (in the non-QE case), as shown in figures 6.10a and 6.10b, respectively. Apart from this bias at low muon energy, the distributions are similar for muon energies above $1.0 \mathrm{GeV}$. The reconstructed hadronic energy in the QE sample is forced by definition to be less than $0.2 \mathrm{GeV}$, as shown in figure 6.10c. The reconstructed hadronic energy in the non-QE sample is shifted slightly toward energies lower than the truth, as shown in figure 6.10d, due to a bias for large hadronic energies to be reconstructed slightly lower in energy.

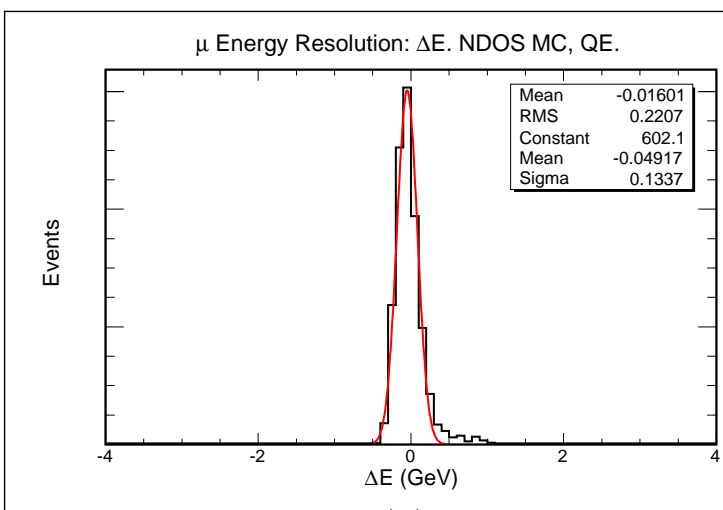

(a)

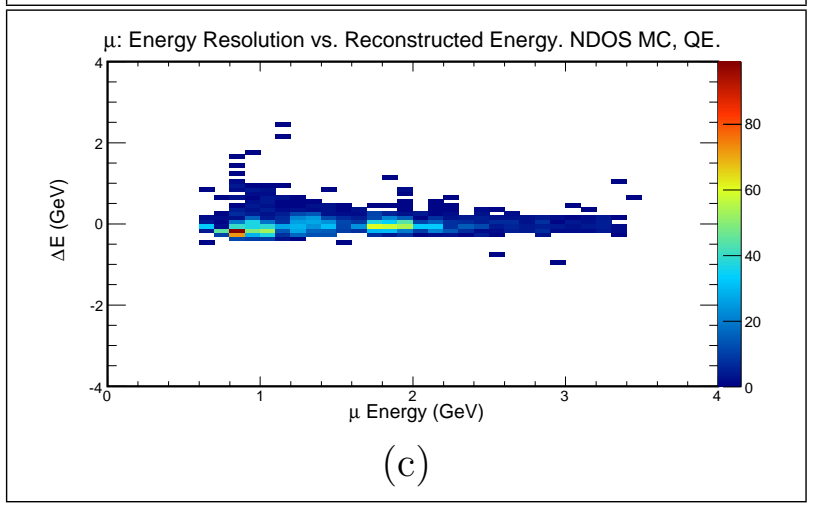

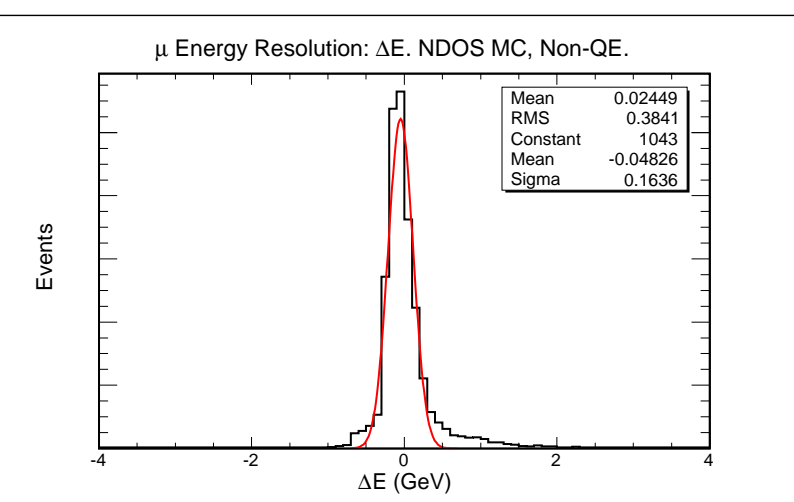

(b)

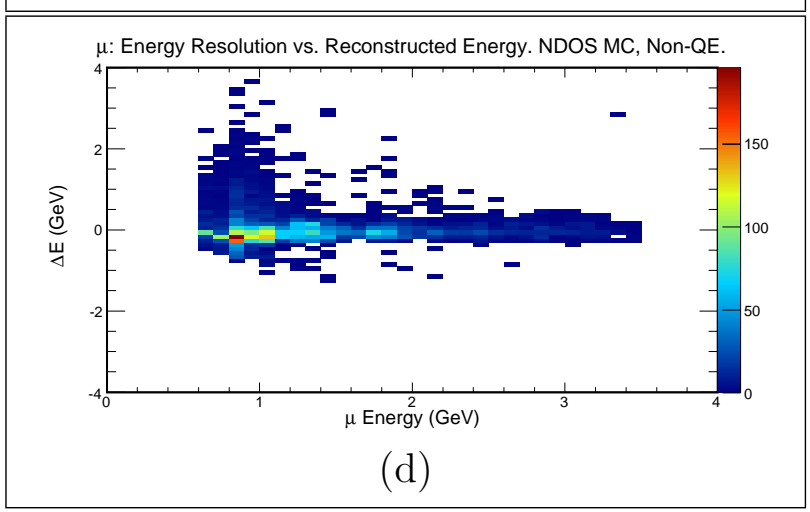

Figure 6.11 Muon Energy Resolutions For QE And Non-QE Events. Overall (a) QE and (b) non-QE energy resolutions. Muon (c) QE and (d) non-QE true minus reconstructed energy $(\Delta E)$ as a function of muon reconstructed energy. The average $\Delta E$ are effectively zero. MC simulation. 
Following the procedure presented in section 6.2 gives the muon and hadronic energy resolutions. The muon energy resolution for the $\mathrm{QE}$ sample at $1 \mathrm{GeV}$ is $150 \mathrm{MeV}(15 \%)$ and at $2 \mathrm{GeV}$ is $100 \mathrm{MeV}(5 \%)$. The overall muon energy resolution for the $\mathrm{QE}$ sample is $130 \mathrm{MeV}$, as shown in figure 6.11a. The muon energy resolution for the non-QE sample at $1 \mathrm{GeV}$ is $150 \mathrm{MeV}(15 \%)$, and at $2 \mathrm{GeV}$ is $130 \mathrm{MeV}(6.5 \%)$. The overall muon energy resolution for the non-QE sample is $160 \mathrm{MeV}$, as shown in figure 6.11b. The true minus reconstructed muon energies as a function of reconstructed muon energy are shown in figure 6.11c, for QE, and in figure 6.11d, non-QE events.

A comparison between figures $6.3 \mathrm{a}$ and $6.11 \mathrm{~d}$ shows that the muon energy resolution around $0.8 \mathrm{GeV}$ comes from non-QE muons that escape the detector, but are reconstructed as contained. This effect was discussed in section 4.3.2. These muons deviate from the original neutrino direction due to the high $y$ of the event.

The hadronic energy resolutions in the QE and non-QE samples are different, as shown in figure 6.12. The overall hadronic energy resolution is: $60 \mathrm{MeV}$ for the QE sample, as shown in figure 6.12a, and $240 \mathrm{MeV}$ for the non-QE sample, as shown in figure 6.12b. Both hadronic energy resolutions, at the average energy deposition in each case, are $30 \%$.
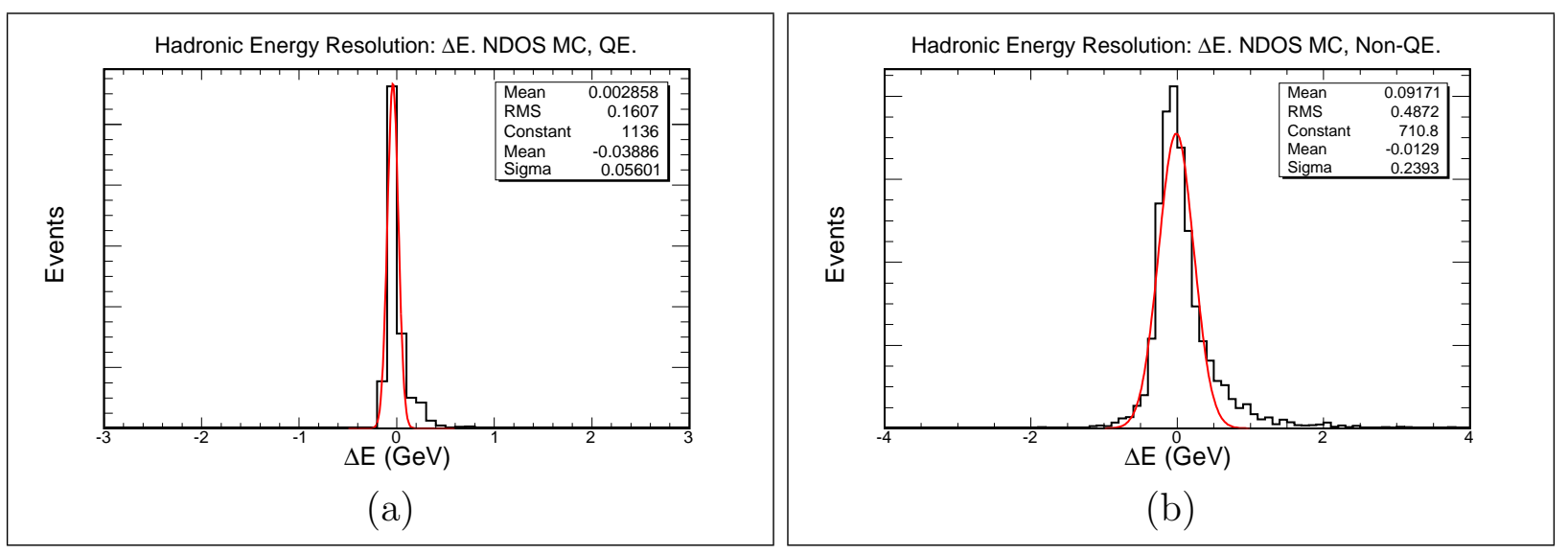

Figure 6.12 Overall Hadronic Energy Resolutions. (a) QE and (b) non-QE events. MC simulation. 


\subsection{Neutrino Energy Estimation}

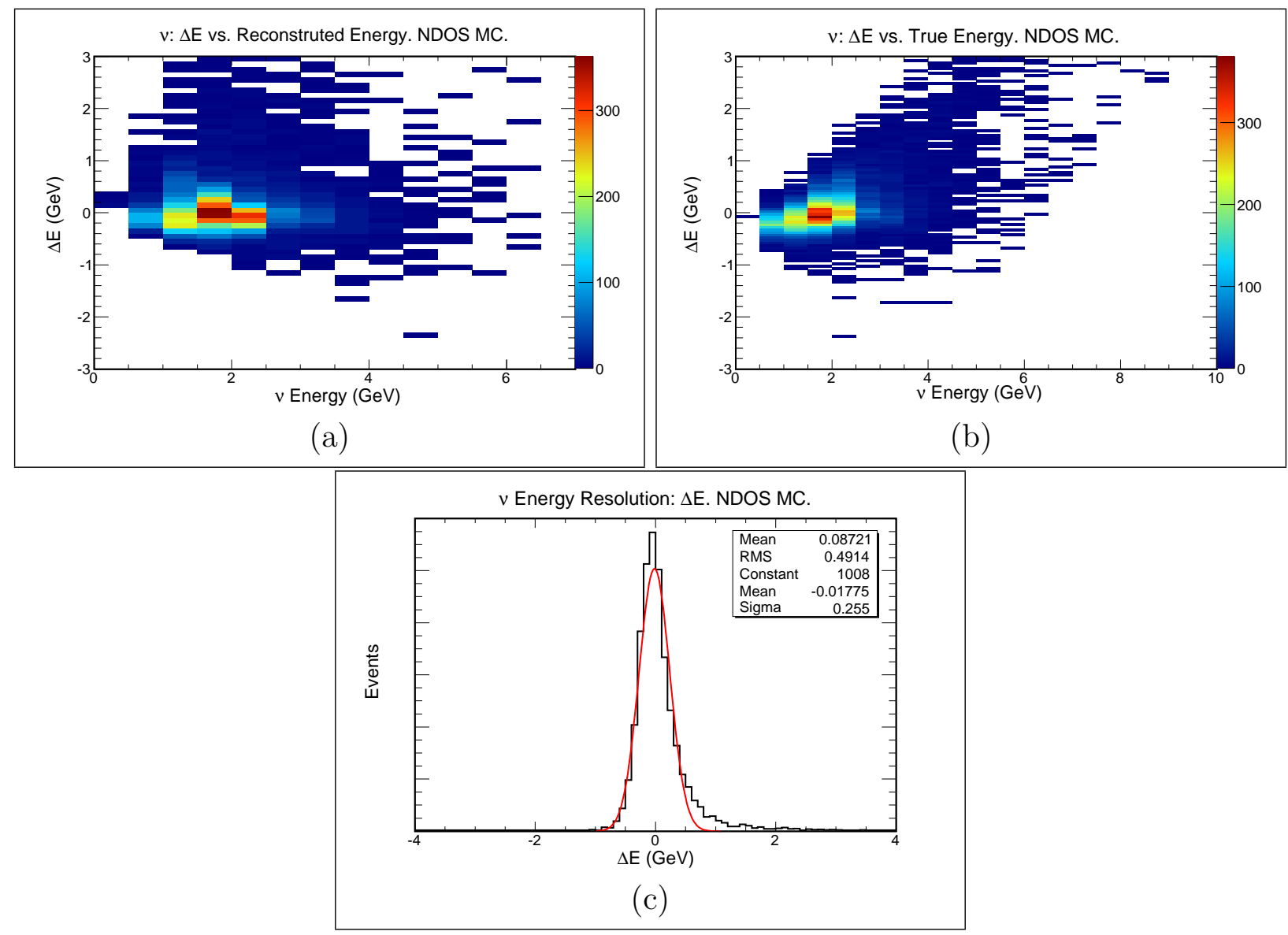

Figure 6.13 Neutrino Energy Resolution. Neutrino true minus reconstructed energy $(\Delta E)$ as a function of the (a) reconstructed (the average $\Delta E$ is effectively zero) and (b) true neutrino energy. (c) Overall neutrino energy resolution. MC simulation.

The reconstructed neutrino energy $\left(E_{\nu}\right)$ in each event is the sum of the two previously estimated energies:

$$
E_{\nu}=E_{R}+E_{\mathrm{had}}+\mathrm{C}_{u}\left(E_{R}+E_{\mathrm{had}}\right),
$$

where $\mathrm{C}_{u}$ are small corrections, of the order of $3 \%$ of the neutrino energy, per neutrino energy bin, introduced in order to achieve the difference between true neutrino energy and reconstructed neutrino energy centered at zero, as seen in figure 6.13a. At the peak of the neutrino energy distribution, near $2.0 \mathrm{GeV}$, the neutrino energy resolution is $200 \mathrm{MeV}(10 \%)$. 
The neutrino energy resolution as a function of the true neutrino energy is presented in figure $6.13 \mathrm{~b}$, where the effect of uncontained muons reconstructed as contained is more evident for energies higher than $3 \mathrm{GeV}$. The overall neutrino energy resolution is $255 \mathrm{MeV}$, as shown in figure $6.13 \mathrm{c}$. The tails account for about $5 \%$ of all events.

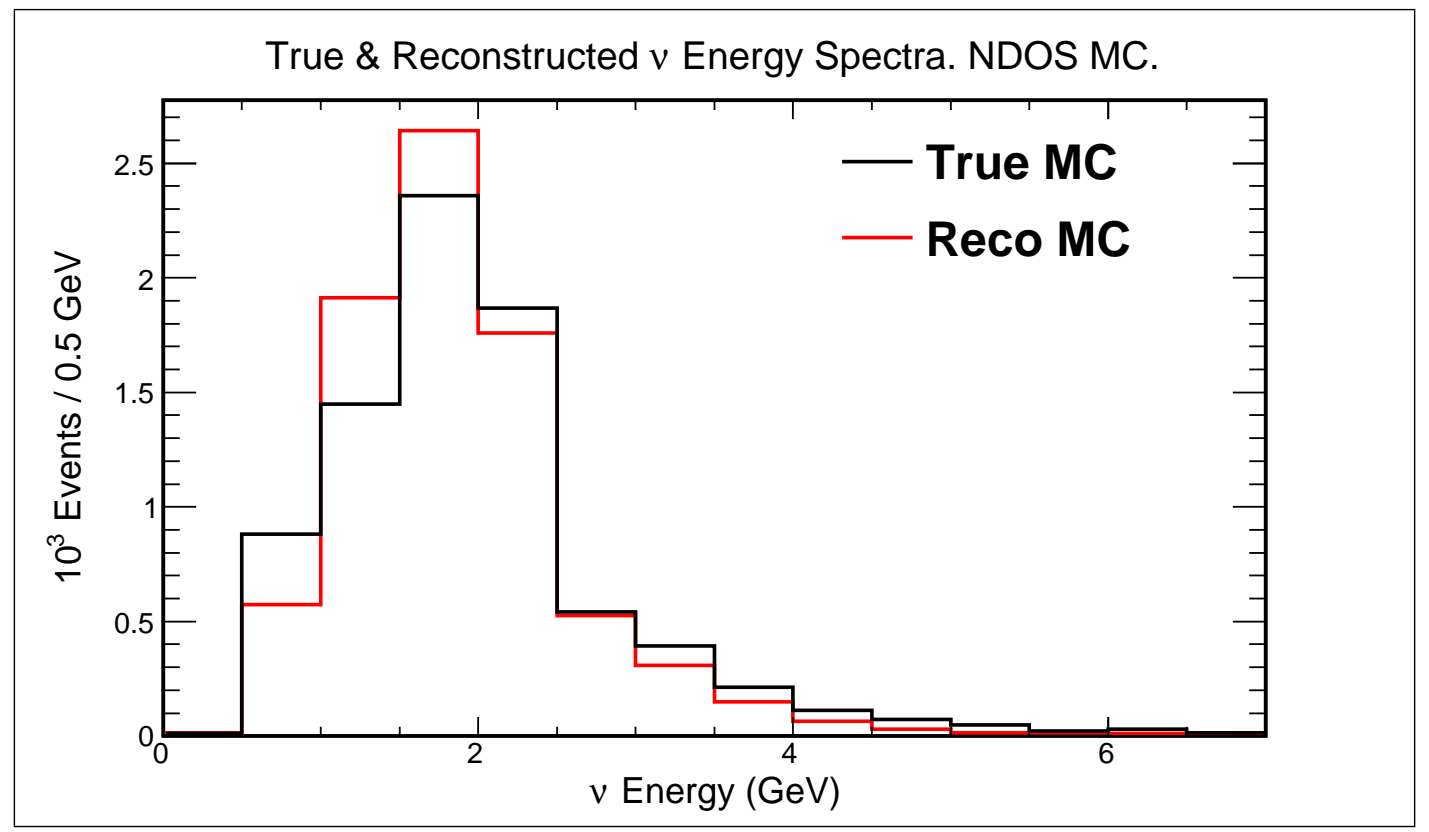

Figure 6.14 Neutrino Energy Distributions. The neutrino true (black) and reconstructed (red) energy distributions. Both distributions have the same number of events. MC simulation.

The neutrino energy distributions, with bins of $500 \mathrm{MeV}$, are in reasonable agreement, as shown in figure 6.14. The effect observed earlier of muons below $0.6 \mathrm{GeV}$ being reconstructed at higher energies is reflected in the movement of events with true neutrino energies between $0.5 \mathrm{GeV}$ and $1.0 \mathrm{GeV}$ to higher values in the reconstructed neutrino energy. Also, inefficiencies in the containment of muons account for the underestimation of the neutrino energies for events with energies higher than $3 \mathrm{GeV}$. The tendency observed earlier to reconstruct hadronic energies above $2.0 \mathrm{GeV}$ to slightly lower values contributes as well. Those events are reconstructed between the $1 \mathrm{GeV}$ and the $2 \mathrm{GeV}$ energy bins. 

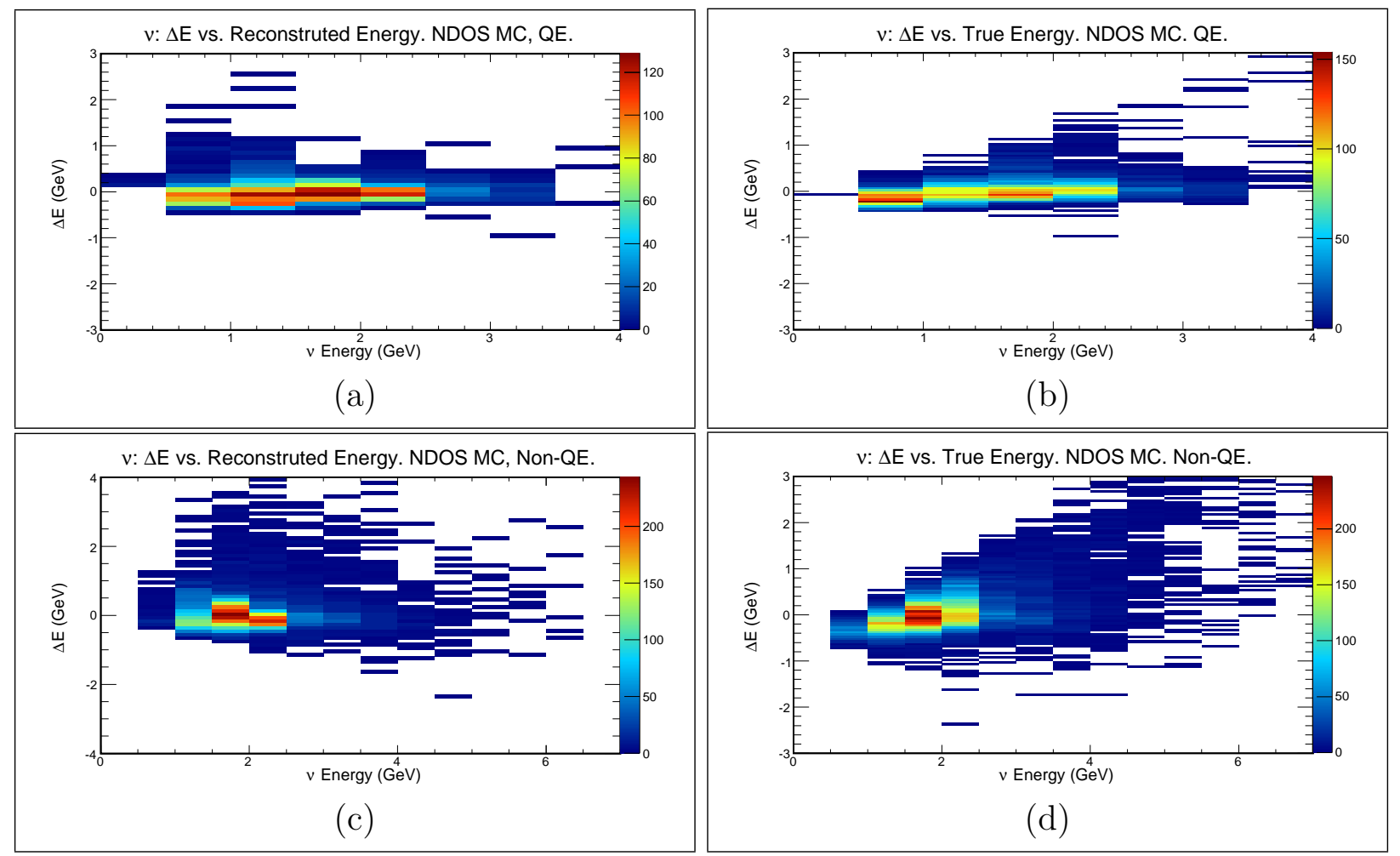

Figure 6.15 QE And Non-QE True Minus Reconstructed Neutrino Energies. QE true minus reconstructed neutrino energy $(\Delta E)$ as a function of (a) reconstructed and (b) true neutrino energy. Non-QE $\Delta E$ as a function of (c) reconstructed and (d) true neutrino energy. MC simulation. 
From the separation of the neutrino sample between QE and non-QE events done in section 6.3 , the neutrino energy resolution, as a function of the reconstructed neutrino energy, are as shown in figures $6.15 \mathrm{a}$ and $6.15 \mathrm{c}$ for the $\mathrm{QE}$ and non-QE samples respectively. The neutrino energy resolution at $2 \mathrm{GeV}$ is: $130 \mathrm{GeV}$ (6.5\%) for the QE sample, and $220 \mathrm{GeV}$ (10.1\%) for the non-QE sample. The neutrino energy resolution as a function of the true neutrino energy are shown in figures $6.15 \mathrm{~b}$ and $6.15 \mathrm{~d}$ for the $\mathrm{QE}$ and non-QE samples respectively. From these figures, the effect of uncontained muons reconstructed as contained is evident in the non-QE. These muons come from high $y$ events, and their angles deviate from the original neutrino angle, therefore the muons escape the detector before stopping.
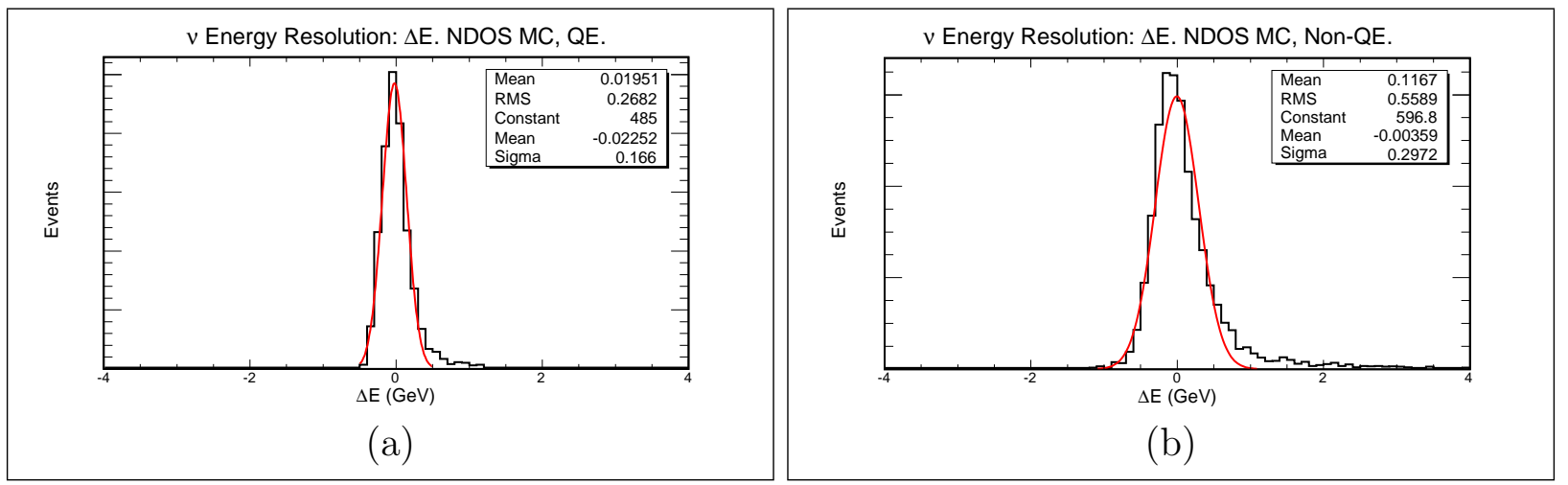

Figure 6.16 QE And Non-QE Neutrino Energy Resolutions. Overall (a) QE and (b) non-QE neutrino energy resolution. MC simulation.

The overall neutrino energy resolution for the $\mathrm{QE}$ sample is $170 \mathrm{MeV}$, as shown in figure 6.16a; and for the non-QE sample it is $300 \mathrm{MeV}$, as shown in figure 6.16b. The tails in figure 6.16 account for about $5 \%$ of the events. The three neutrino energy resolutions, as a function of the reconstructed neutrino energy, are compared in figure 6.17.

The neutrino energy distributions for QE and non-QE are presented in figure 6.18. In these figures the true $\mathrm{MC}$ distributions represent the $\mathrm{QE}$ and non-QE interactions as defined in the MC simulation. The reconstructed MC distributions represent the interactions as 


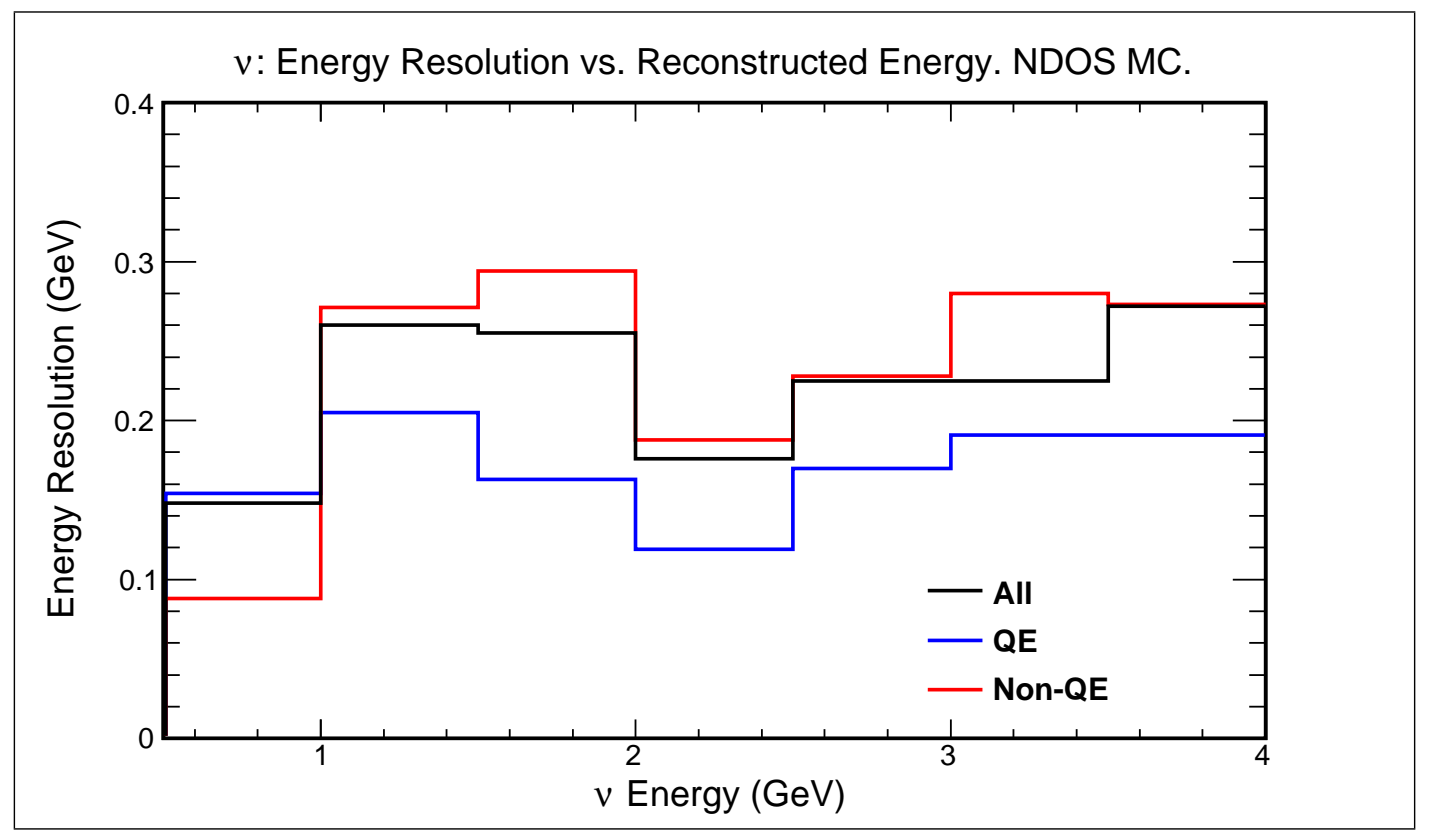

Figure 6.17 Comparison Of Neutrino Energy Resolutions. The neutrino energy resolutions for all (black), QE (blue), and non-QE events. MC simulation.
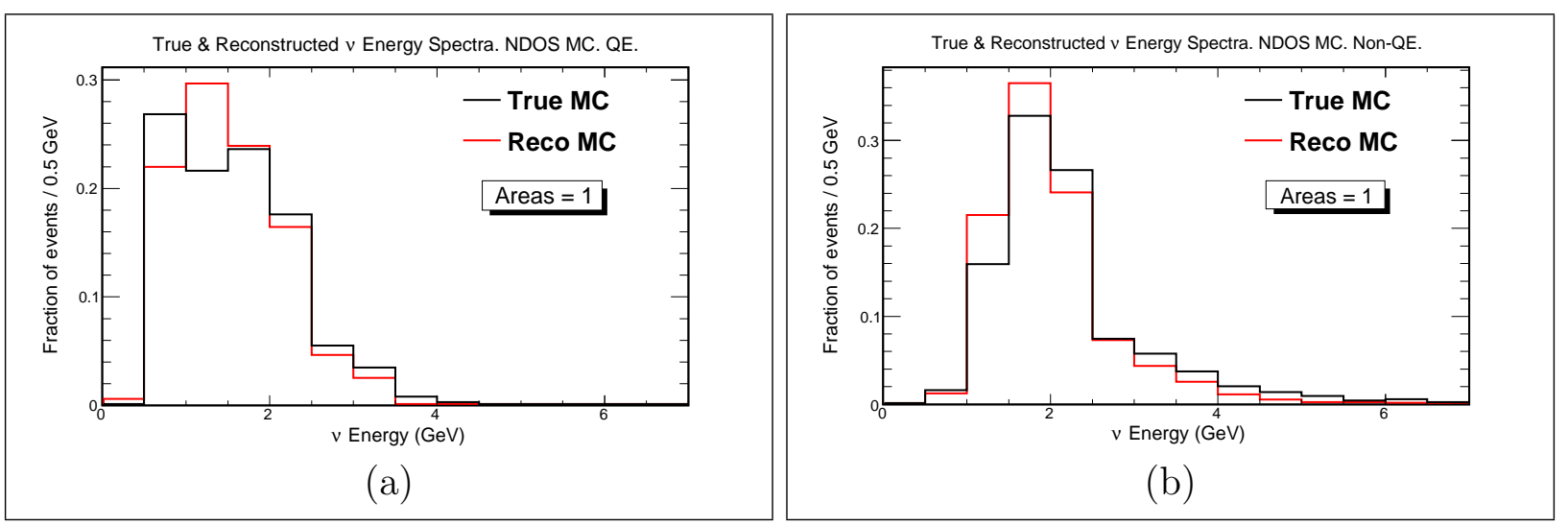

Figure 6.18 QE And Non-QE Neutrino Energy Distributions. (a) QE and (b) non-QE neutrino true (black) and reconstructed (red) energy distributions. Areas normalized to 1 in order to compare the shapes of the distributions. MC simulation. 
defined by relations (6.6). In the QE and non-QE interactions, the area normalized true and reconstructed neutrino energy distributions are in reasonable agreement. The distributions for the QE and non-QE are distinct. The QE, with energy mean 28.4\% smaller than that of the non-QE, slowly varies between $0.5 \mathrm{GeV}$ and $2.5 \mathrm{GeV}$, as shown in figure 6.18a. The non-QE distribution is strongly peaked near $2.0 \mathrm{GeV}$, as shown in figure $6.18 \mathrm{~b}$. These effects are the consequence of the cross sections dependence on the neutrino energy, as was shown in figure 2.4. The two cross sections are equal (for $\nu_{\mu}$ ) at about $1 \mathrm{GeV}$; at this point $\mathrm{QE}$ is reaching a plateau and non-QE is increasing. The event rate in figure 6.18 is dominated by non-QE for energies higher than $1 \mathrm{GeV}$.
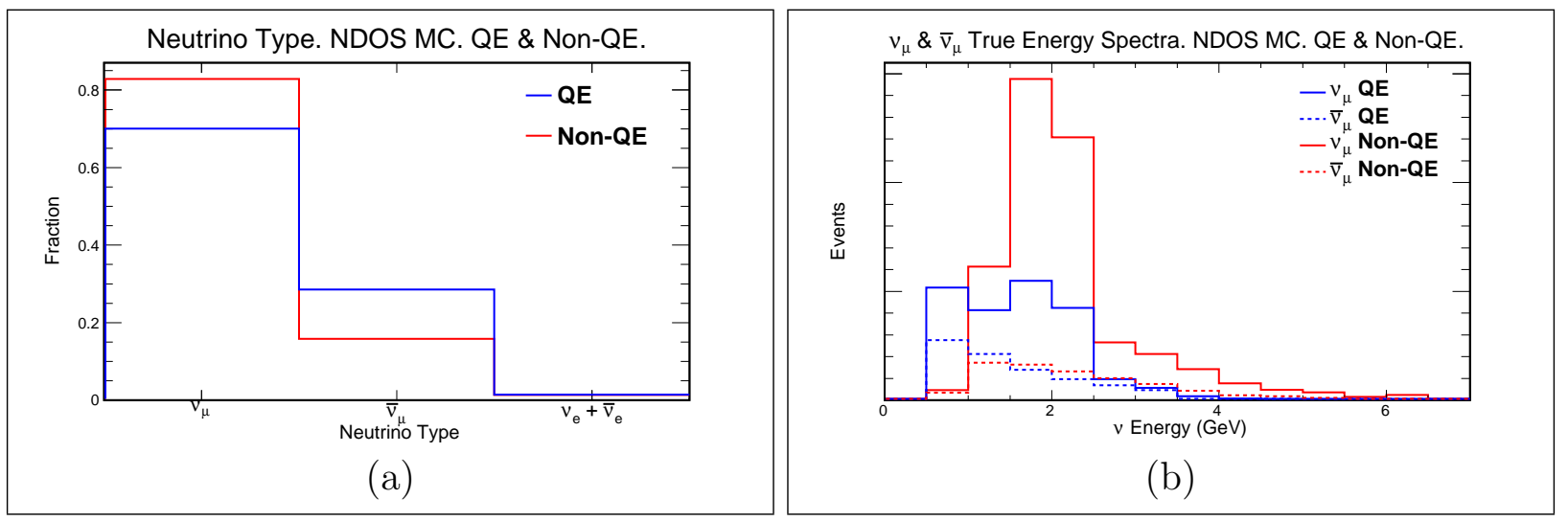

Figure 6.19 Neutrino Types: Fractions And Energy Distributions. (a) Fraction of neutrino types discriminated by the interaction type. (b) Neutrino energy distributions discriminated by interaction type and neutrino type. No scale shown in order to compare the shapes. MC simulation.

Each of the two interaction types is the sum of $\nu_{\mu}$ and $\bar{\nu}_{\mu}$ components. Figure 6.19 presents this composition. The overall fraction of the different neutrino types discriminated by interaction type is presented in figure 6.19a. The non-QE fraction is higher for interactions generated by a $\nu_{\mu}$, and the $\mathrm{QE}$ fraction is higher ${ }^{2}$ for interactions generated by a $\bar{\nu}_{\mu}$. The neutrino energy distributions per neutrino type and interaction type are shown in figure

\footnotetext{
${ }^{2}$ This is true because $\bar{\nu}_{\mu}$ interacts with Hydrogen nuclei in QE interactions and the $\nu_{\mu}$ does not.
} 
6.19b. The $\bar{\nu}_{\mu}$ contribution at the $2 \mathrm{GeV}$ peak is about 10\%. This contribution almost triples at adjacent lower and higher energy bins from the peak, as a result of the various cross sections that originate the interactions. 


\section{Chapter 7}

\section{Systematic Uncertainties}

Systematic uncertainties are often related to measuring devices or measuring methods used in experiments. A measuring device, or a detector, could consistently read the value of a measured quantity with an offset from its real value. This offset could be due to an scale factor, a calibration issue, or the materials and geometry of the detector. A measuring method could under or over estimate a physical quantity due to the way it is designed or implemented, which would result in a measurement that is consistently different from the real value of the measured quantity.

There are six relevant systematic uncertainties on the number of events identified during this analysis. The energy estimation of the neutrino candidate event relies on the absolute energy calibration of the detector. Differences in the calibrations between data and MC simulated events result in systematic uncertainties in the number of selected events per energy bin. The various channels configurations of the detector during the period in which the data was taken introduce systematic uncertainties in the number of selected events since different configurations could disagree on whether a particular event is contained or not in the detector. To minimize this uncertainty, each channels configuration in the data sample was simulated in the MC sample. The uncertainty in the cross sections and the modeled final state physics influence the number of selected events. In the case of the cross sections, the measured number of events is directly proportional to the cross sections; and the model used 
to describe the physics of the final state could increase or decrease the rate at which particular types of events occur under the circumstances of the experiment. The mass of the target region of the detector is also directly proportional to the measured number of events, and therefore its uncertainty creates a systematic uncertainty on the number of selected events. A mathematical procedure known as unfolding (see appendix C) shifts selected events from the energy bins in which these are measured to the most probable energy bin for a particular event. The shift is based on relations between true and reconstructed events that are built into the algorithm. Since the rearrangement of events introduces a change in the number of selected events per energy bin, the unfolding method also introduces a systematic uncertainty on the number of selected events that is related to the parameters of the algorithm that are subject of variation due to optimization criteria. Finally, the counting method of the POT directly affects the relative number of selected events. The same absolute number of selected events changes its interpretation depending on the reported POT. Thus, uncertainties in the POT introduce systematic uncertainties in the number of selected events. Each one of these major systematic uncertainties is presented in the following sections.

\subsection{Energy Estimation Uncertainty}

There are two key features in the energy estimation process: the length of the longest track per event, and the deposited hadronic energy. To determine the systematic uncertainty of the energy estimation of neutrino events in the NDOS, the mean energy deposited per unit length in cosmic tracks are compared to MC simulated muon tracks, as seen in figure 7.1. Differences in the two distributions imply a systematic difference in the estimated energy of measured and simulated neutrinos. The two means of these distributions differ by $9.1 \%$, 


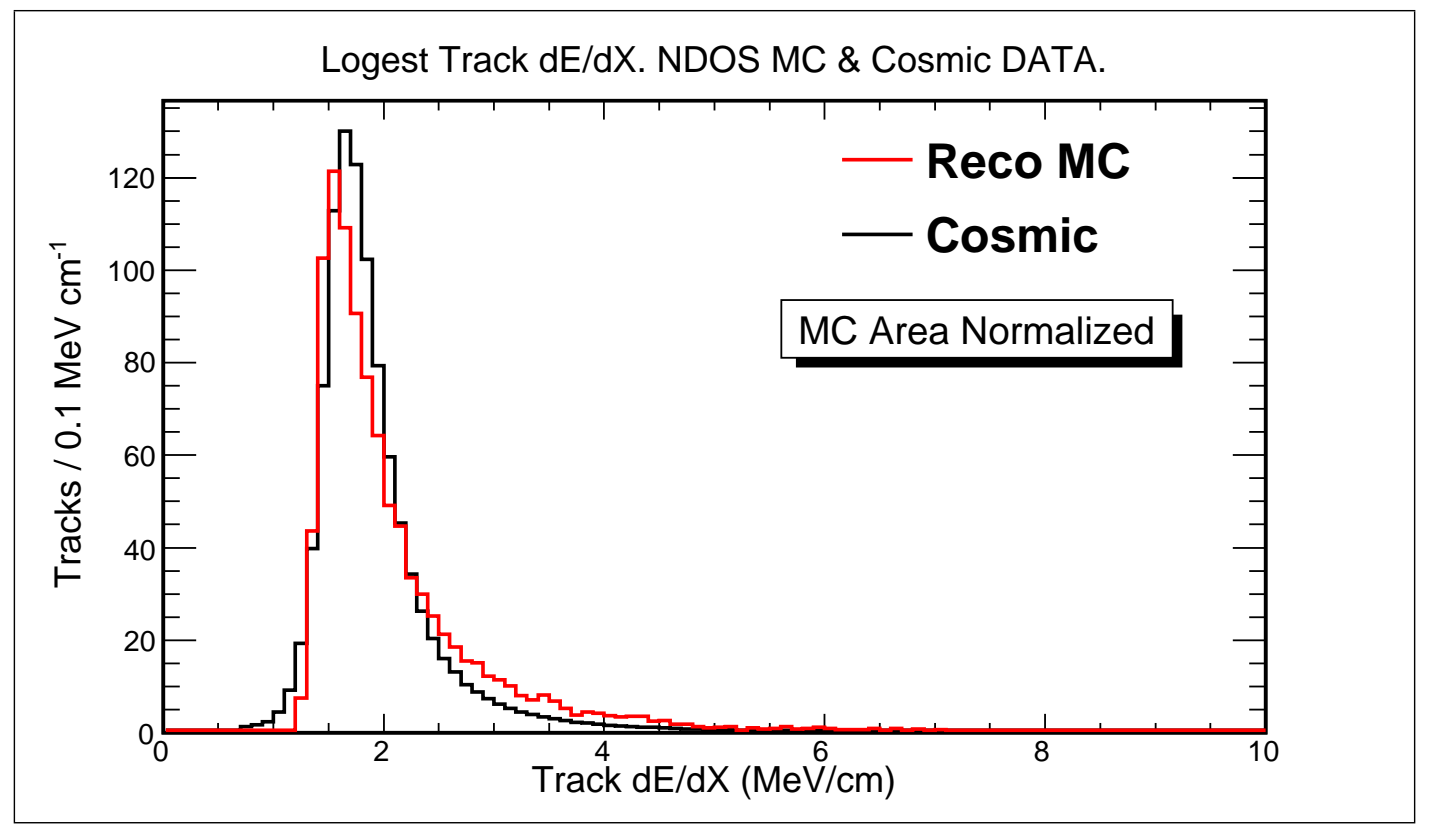

Figure 7.1 Mean Energy Deposition Per Unit Length. $d E / d X$ of cosmic (black) and reconstructed MC simulated tracks (red). The MC simulation area is normalized to data.

which is interpreted as a $10 \%$ systematic uncertainty on the deposited energy.

This $10 \%$ systematic uncertainty is added to and subtracted from the deposited energy in equation (6.5) to find how the hadronic energy estimation changes. These changes affect the MIP fraction of the tracks as well, which is taken into account when evaluating the change in the number of events. Finally, these systematic uncertainty affects the QE and non-QE separation since it is carried out using the estimated energy of the hadronic system.

The muon energy estimation comes from the measured track length using equation (6.2). The comparison between the longest track lengths of reconstructed MC simulated and data tracks is presented in figure 7.2. The mean of the MC simulated track length distribution is 9.6\% larger than that of data. This difference is assumed as a $10 \%$ systematic uncertainty on the track length measurement. This $10 \%$ systematic uncertainty is added to and subtracted from the track length in equation (6.2) to find how the muon energy estimation changes. 


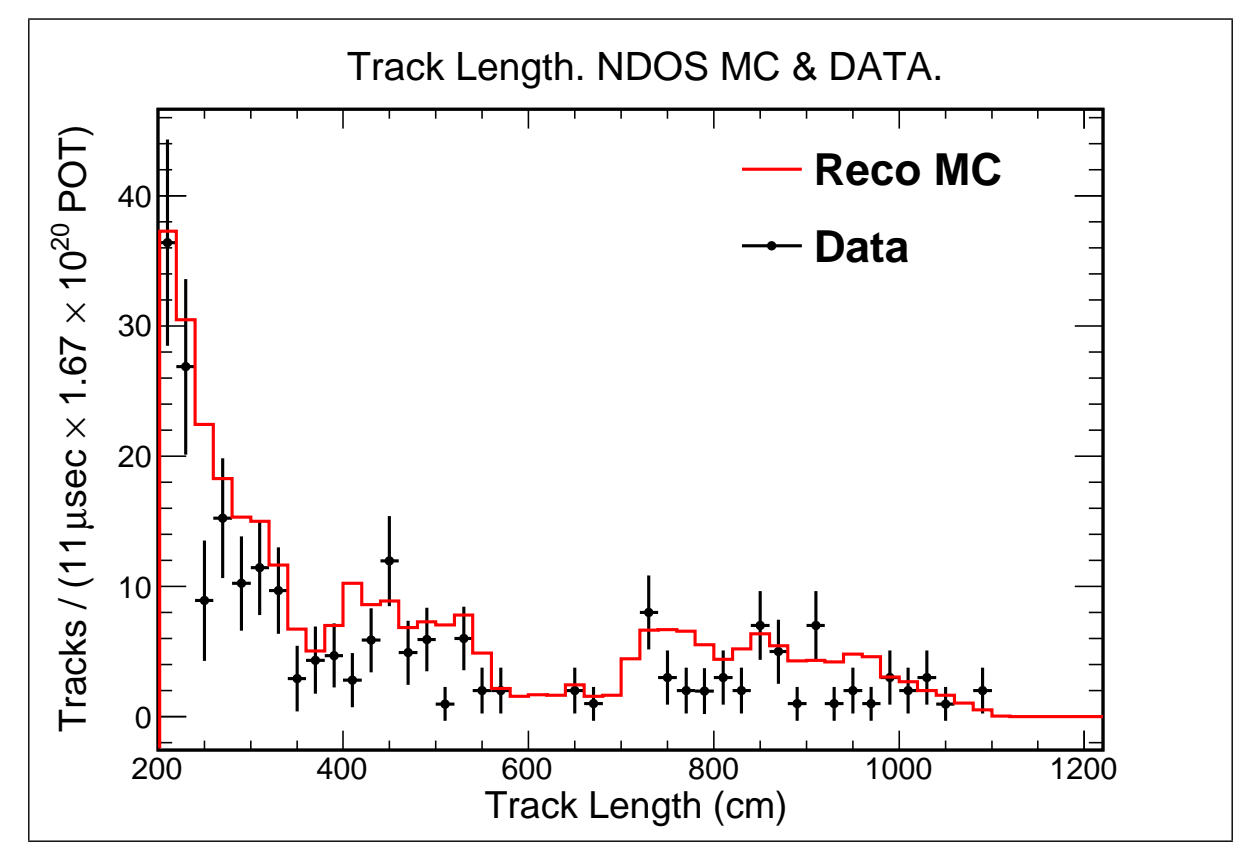

Figure 7.2 Comparison Of Data And MC Simulated Longest Track Length Distributions. Reconstructed MC simulated (red) and data (black) tracks.

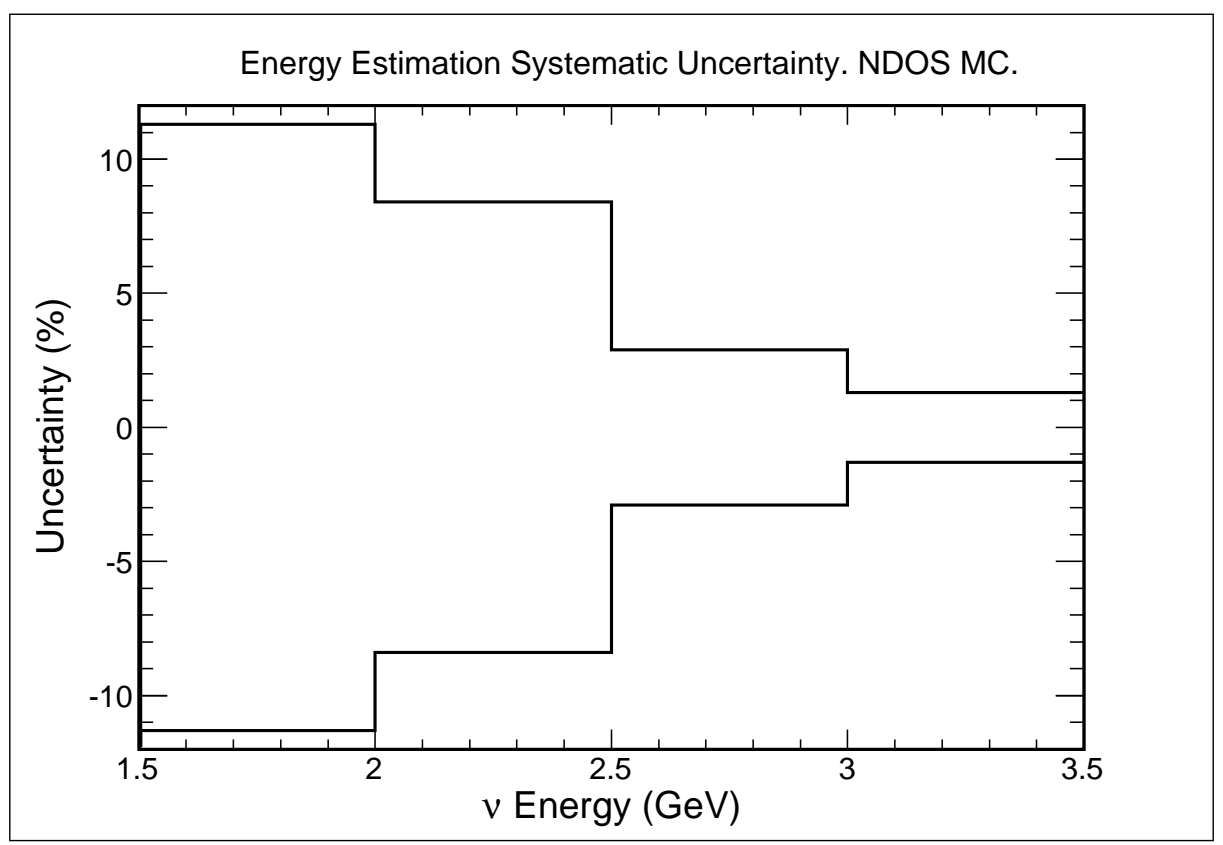

Figure 7.3 Energy Estimation Systematic Uncertainty As A Function Of The Neutrino Energy. 
These changes combined with those presented above, for the deposited energy, give the systematic uncertainties $^{1}$ to the number of events shown in figure 7.3 . The impact of these variations $(\delta E)$ is of the order of $8 \%$.

\subsection{Prototype Detector's Channels Configuration Un- certainty}

The NDOS active channels configuration varied over time, since APDs were added, removed, and shifted during the period when the data were taken. To estimate the systematic uncertainties introduced to the number of events due to these changes in the channels configuration, three different configurations are examined (refer to figure 4.23): run numbers smaller than 13250, run numbers bigger than 13250 and smaller than 13600, and run numbers bigger than 13600. From the first set, the channels configuration of run 13220 is chosen because it has the least number of active channels. From the second set, the channels configuration of run 13258 is chosen because it is the one with the most active channels. Finally, from the third set, the channels configuration of run 13703 is chosen because it has the most active channels.

A set of MC simulated events is reconstructed with each of the three channels configurations, and the number of reconstructed events is examined as a function of the run number. The systematic uncertainties ${ }^{2}$ on the number of events due to the impact of the various channels configurations are shown in figure 7.4. The same event reconstructed under different channels configurations could be selected as contained in some of them, and not selected in

\footnotetext{
${ }^{1}$ Figure 7.3 presents the uncertainties as percentages of the total number of events.

${ }^{2}$ Figure 7.4 presents the uncertainties as percentages of the total number of events.
} 


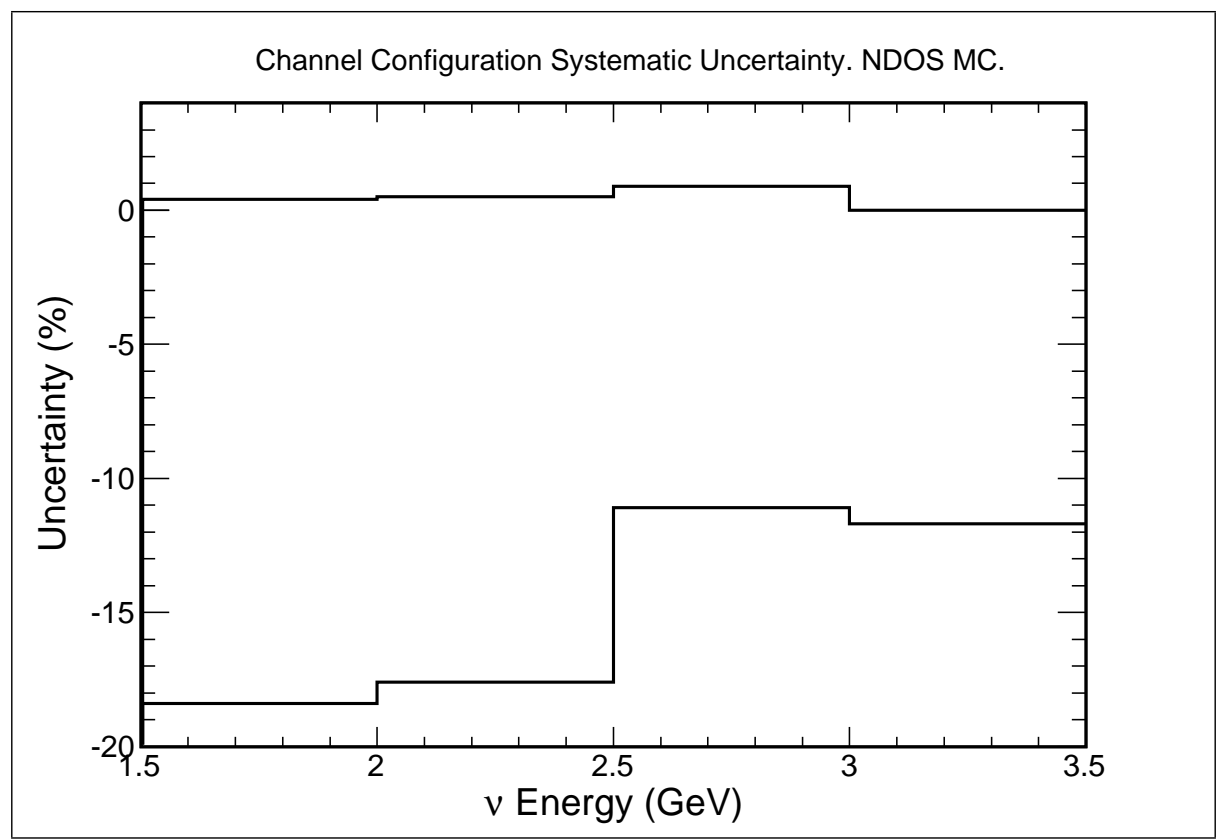

Figure 7.4 Channels Configurations Systematic Uncertainty As A Function Of The Neutrino Energy.

others because it was not reconstructed as contained. The lack of instrumented cells in key points of the detector is responsible for this issue. The variation in the number of selected events introduced by each channels configuration is compared to the nominal one, and the maximum variation is used as the systematic uncertainty. The impact of these variations $(\delta C)$ is of the order of $15 \%$.

\subsection{GENIE Cross Sections And Final State Physics Uncertainty}

In order to give the users the ability to verify the validity of new models explaining nuclear interactions, or study how possible variations to the current models could affect the physics output of the simulations, GENIE has features that allow the users to introduce variations to 
key parameters that will result in changes to the simulated outputs produced by the software. The developers provided a mechanism to assign weights to simulated neutrino interactions, which is implemented by the ReWeight package. The main concept behind this process is that for each input physics quantity $P$, with uncertainty ${ }^{3} \delta P$, which is taken into account, there is a parameter $x_{P}$ such that:

$$
P \rightarrow P^{\prime}=P\left(1+x_{P} \frac{\delta P}{P}\right)
$$

$P$ could be a single physical parameter, a simple function, or a prediction done by a MC simulation. Some of these quantities may not be easy to write analytically or tabulated, therefore it is preferable to formulate the problems in terms of $x_{P}$. The parameter $x_{P}$ is an integer, positive and negative values are allowed. When $x_{P}=0$, there would be no variation to $P . x_{P}= \pm 1$ indicates that $P$ would change by one standard deviation, $\delta P[109]$.

The reweighting package is named according to the final effect that it produces: it assigns weights to individual MC simulated events based on the changes introduced by the $x_{P}$. The tool is very useful since it simplifies the analysis. Instead of running the MC simulation as many times as changes are introduced to the models, the weights reflect the importance of a given event on the sought final result. A weight equal to 1 means that the variation in a given $x_{P}$ had no effect in the final result. A weight greater than 1 indicates that the variation in $x_{P}$ produces an increase in the frequency of such weighted events proportional to the size of the weight. A weight less than 1 means that the variation in $x_{P}$ produces a decrease in the frequency of such weighted events proportional to the size of the weight. The $M_{a}^{\text {ccqe }}$ parameter (defined in table 7.1) is used as an example of the ReWeight package

\footnotetext{
${ }^{3} \delta P$ is the estimated standard deviation of $P$.
} 


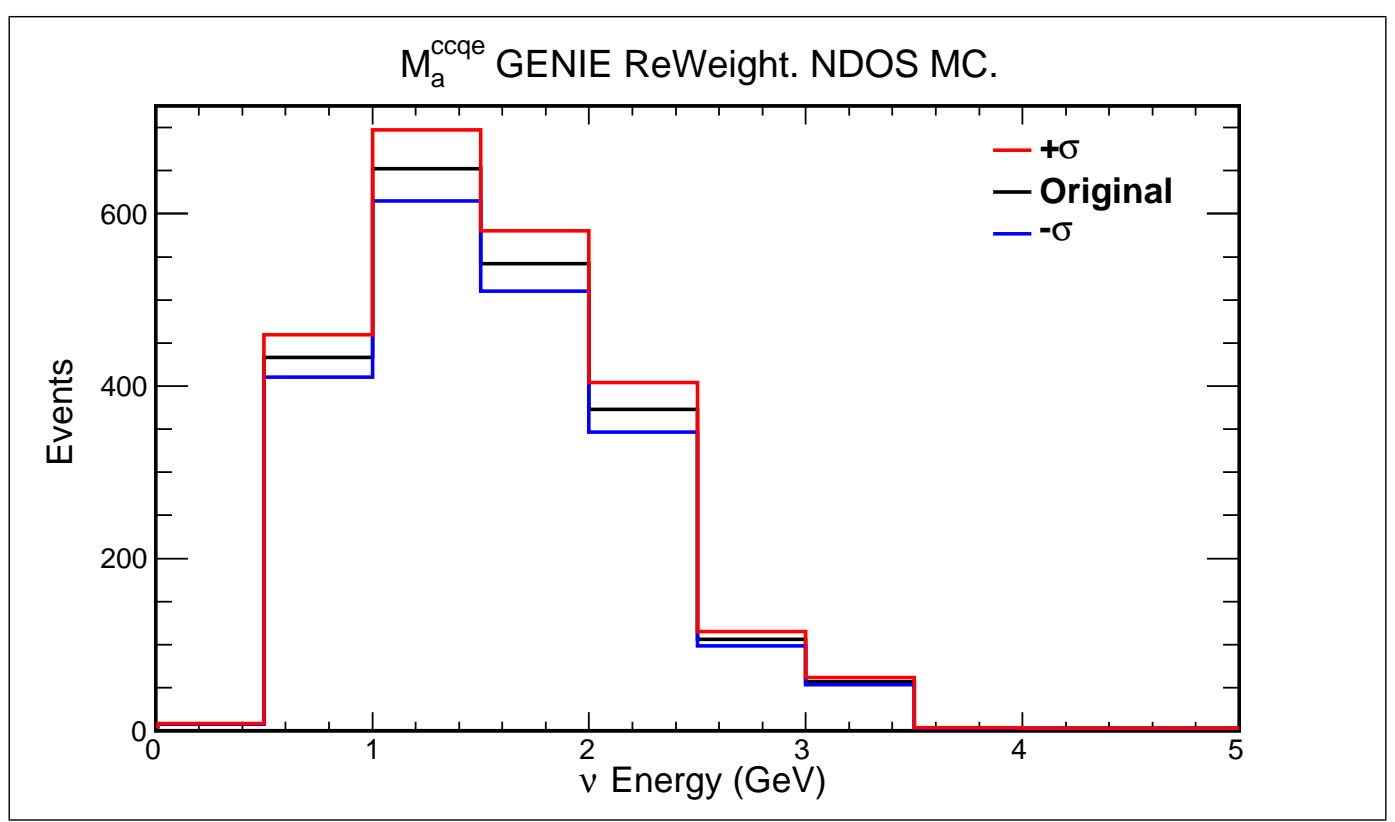

Figure 7.5 Example Of The Re Weight Package Output. The black distribution is the original, unaltered, MC sample. The $+1 \sigma$ variation to $M_{a}^{\text {ccqe }}$ is shown in red, and the $-1 \sigma$ variation in blue.

output, which is presented in figure 7.5. The original neutrino energy spectrum features a MC simulated sample of events that passed all the event selection cuts. The ReWeight package assigned weights to each of the events in the original distribution, and the consequences of such weights are seen in red for the $+1 \sigma$ variation, and in blue for the $-1 \sigma$ variation. Although the number of entries in the three distributions is the same, the integrals in the reweighted distributions change as a result of the applied weights by $7.2 \%$ for $+\sigma$, and by $6.0 \%$ for $-\sigma$. The percentage of variation in the number of events is used as the systematic uncertainty for this particular parameter. This procedure is the same for all the studied parameters.

The dominant parameters that affect this analysis, include the axial mass for $\mathrm{CC}$ QE scattering, the nuclear Pauli suppression effects in CC QE reactions ${ }^{4}$, the non-resonance

\footnotetext{
${ }^{4}$ Modifying the Fermi momentum is the way to affect the Pauli suppression.
} 


\begin{tabular}{|c|c|c|}
\hline$x_{P}$ & Description of $P$ & $\delta P / P$ \\
\hline \hline & $\nu_{\mu}$ interaction cross section systematic parameters & \\
$\mathrm{M}_{a}^{\text {ccqe }}$ & Axial mass for CC QE & $+25 \%-15 \%$ \\
$\mathrm{M}_{a}^{\text {ccres }}$ & Axial mass for CC resonance neutrino production & $\pm 20 \%$ \\
$\mathrm{R}_{\mathrm{bkg}}^{\nu n c c 1 \pi}$ & Non-resonance bkg in $\nu$ CC $1 \pi$ reactions & $\pm 50 \%$ \\
\hline & Hadronization systematic parameters & \\
$\mathrm{fz}$ & Hadron formation zone & $\pm 50 \%$ \\
\hline & Intranuclear hadron transport systematic parameters & \\
$\mathrm{MFP}_{\pi}$ & $\pi$ mean free path (total rescattering probability) & $\pm 20 \%$ \\
$\mathrm{MFP}_{n}$ & Nucleon mean free path (total rescattering probability) & $\pm 20 \%$ \\
$\mathrm{Fr}_{\mathrm{inel}}^{n}$ & Nucleon inelastic reaction probability & $\pm 40 \%$ \\
$\mathrm{Cex}_{n}$ & Nucleon charge exchange probability & $\pm 50 \%$ \\
$\mathrm{Abs}_{n}$ & Nucleon absorption probability & $\pm 20 \%$ \\
$\operatorname{Fr}_{\pi}$ & Nucleon $\pi$ production probability & $\pm 20 \%$ \\
\hline
\end{tabular}

Table 7.1 GENIE Parameters. Taken from [109].

background for all CC $1 \pi$ and $2 \pi$ production channels, CC DIS, intranuclear hadron transport model, and total rescattering probability for hadrons within the target nucleus 5 . Some of these categories have more than one parameter available for the reweighting process. All the parameters tabulated in tables 8.1, 8.2, and 8.3 of [109] are studied in this analysis, however not all of them introduce finite changes to the number of events. All the parameters that introduced finite variations to the number of events are presented in table 7.1, which also shows their standard deviations.

There are three categories in table 7.1: neutrino interaction cross section systematic parameters, intranuclear hadron transport systematic parameters, and hadronization systematic parameters. Hadrons produced in the nuclear environments are not obtained from a single interaction, initially quarks propagate through the nucleus with low probability of interaction as these are not yet part of a hadron. GENIE models this effect by defining

\footnotetext{
${ }^{5}$ This systematic is related to the mean free path of the hadron within the nucleus.
} 


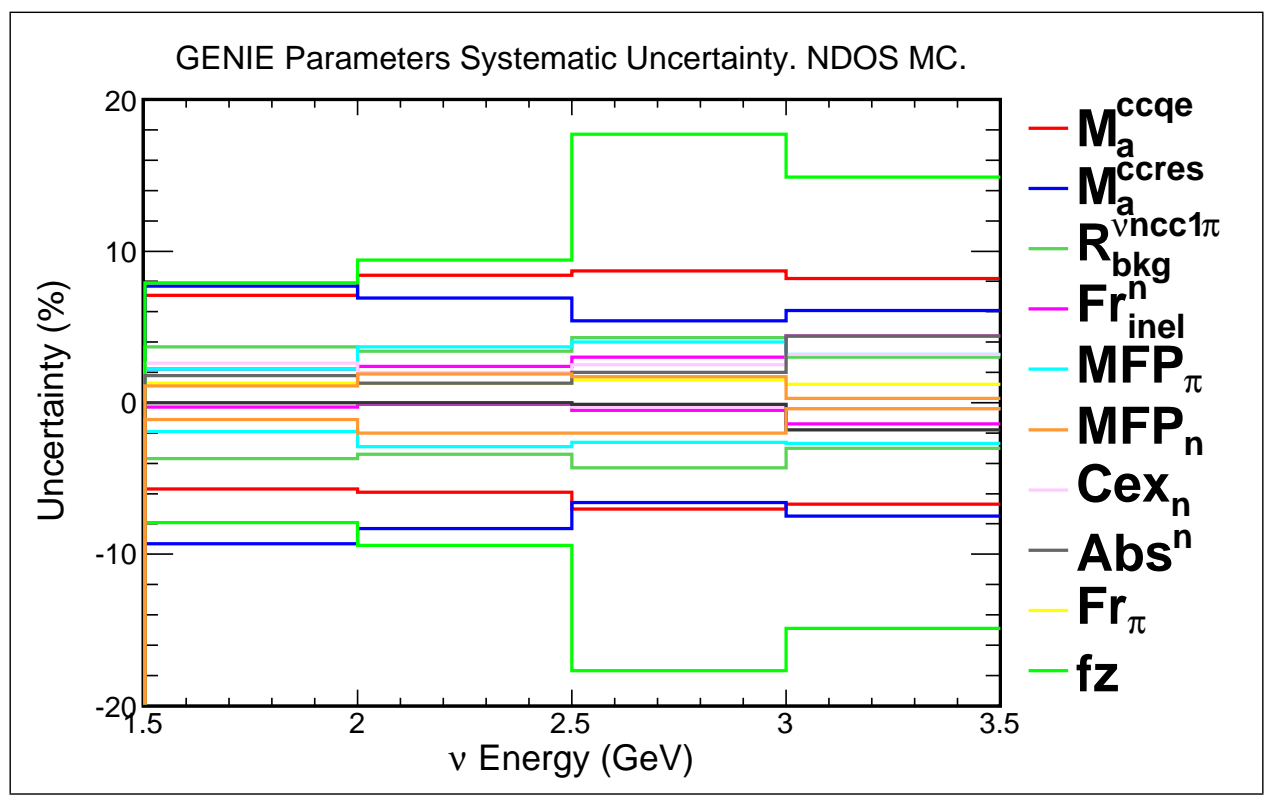

Figure 7.6 GENIE Parameters Systematic Uncertainty As A Function Of The Neutrino Energy. The parameters shown are defined in table 7.1.

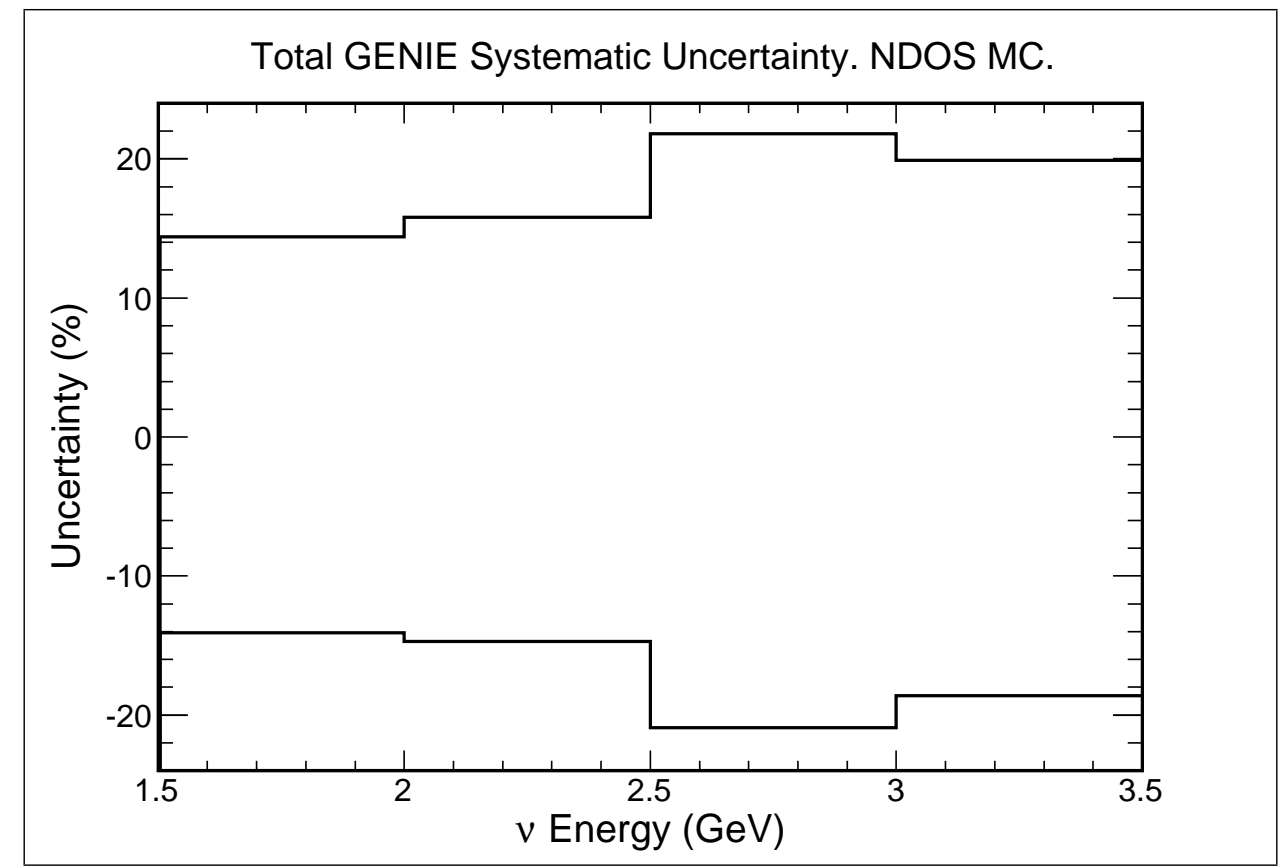

Figure 7.7 GENIE Parameters Total Systematic Uncertainty As A Function Of The Neutrino Energy. The sum of the parameters defined in table 7.1 is done in quadrature. 
a free step, $f_{z}=\mathbf{p} c \tau_{0} / \mathrm{m}$, where $\mathbf{p}$ is the hadron's momentum, $\tau_{0}=0.342 \mathrm{fm} / \mathrm{c}$ is the formation time, $\mathrm{m}$ is the mass of the hadron, and $c$ is the speed of light. The formation zone is defined by the distance between the intranuclear event vertex and the recorder position of the hadron at the beginning of the intranuclear cascade step [109].

The systematic uncertainties ${ }^{6}$ in the number of events obtained for the parameters presented in table 7.1 are summarized in figure 7.6. The impact of the combined variations of all the studied parameters $(\delta G)$ is of the order of $15 \%$, in the energy region where most of the data occurs, i.e. $1.5 \mathrm{GeV}$ to $2.5 \mathrm{GeV}$. The sum of these uncertainties is done in quadrature, and is shown in figure 7.7.

\subsection{Unfolding Systematic Uncertainty}

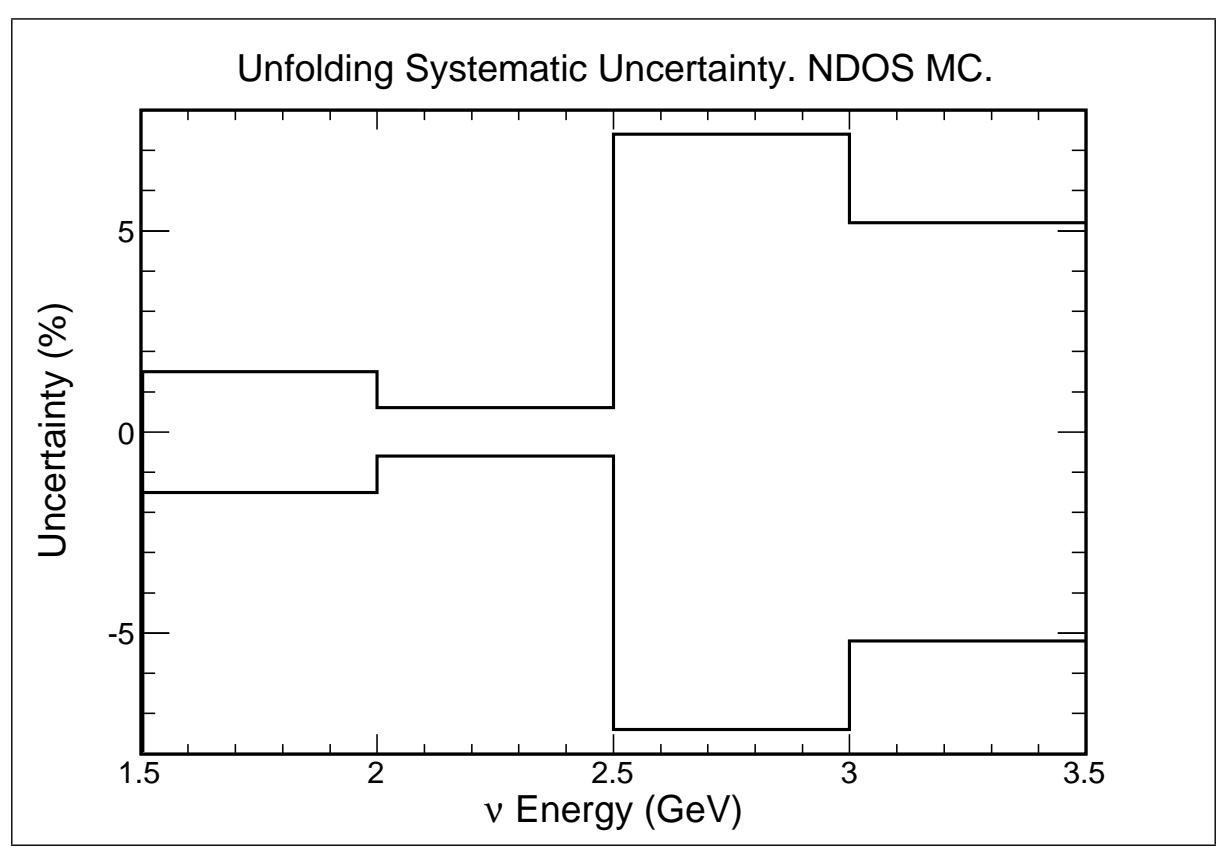

Figure 7.8 Unfolding Algorithm Systematic Uncertainty As A Function Of The Neutrino Energy.

\footnotetext{
${ }^{6}$ Figure 7.6 presents the uncertainties as percentages of the total number of events.
} 
The unfolding procedure presented in appendix $\mathrm{C}$ uses two parameters that can modify the output of the algorithm, hence the number of events would vary accordingly. These parameters are: $k$, which sets the number of statistically significant equations, and $n_{\mathrm{TSVD}}$, which is the number of toy MC simulated events generated by the TSVDUnfold tool to calculate the uncertainties on the unfolded distribution. To find the systematic uncertainty in the unfolded distribution of the number of events, the $k$ parameter is modified to be: 4 and 6 , the original value was 2 ; and the $n_{\mathrm{TSVD}}$ is modified to be: 50 and 200 , the original value was 100 . The systematic uncertainties ${ }^{7}$ in the number of events obtained after varying these two parameters are shown in figure 7.8. The impact of these variations $(\delta U)$ is of the order of $5 \%$.

\subsection{Total Systematic Uncertainty}

Before discussing the total systematic uncertainties, there three more systematic uncertainties to be considered in this analysis: that related to the POT calculation, that related to the fiducial mass, and that related to the fraction of neutrinos resulting from each charged meson decay, i.e., the charged kaons to pions ratio, in the number of selected neutrino candidates. $\mathrm{NO} \nu \mathrm{A}$ uses the same POT counting scheme used by MINOS, thus the $2 \%$ systematic uncertainty $(\delta P)$ used in their study of $\nu_{\mu}$ disappearance [110] is assumed in this analysis. The fiducial mass has a $5 \%$ systematic uncertainty which comes from the comparison between the physical measurements performed on the materials of the detector and the parameters extracted from the MC simulation.

A. Lebedev [111] measured the ratio of charged pions and kaons production by $120 \mathrm{GeV} / \mathrm{c}$

\footnotetext{
${ }^{7}$ Figure 7.8 presents the uncertainties as percentages of the total number of events.
} 
protons incident on a carbon target. The production ratios were measured in longitudinal momenta from $20 \mathrm{GeV} / \mathrm{c}$ to $90 \mathrm{GeV} / \mathrm{c}$. These are not the longitudinal momenta of the mesons involved in the NDOS measurement, however, the lack of measurements in the longitudinal momentum region concerning this analysis (around $7 \mathrm{GeV} / \mathrm{c}$ for kaons) leads to use the measurements in [111] as a constraint on the $K / \pi$ ratio at the momentum region of interest. The ratios in [111] are in agreement with the ratios embedded in FLUKA within 10\%. This value is used as the systematic uncertainty on the number of neutrinos coming from charged pion decays $(\delta R)$ in this analysis.

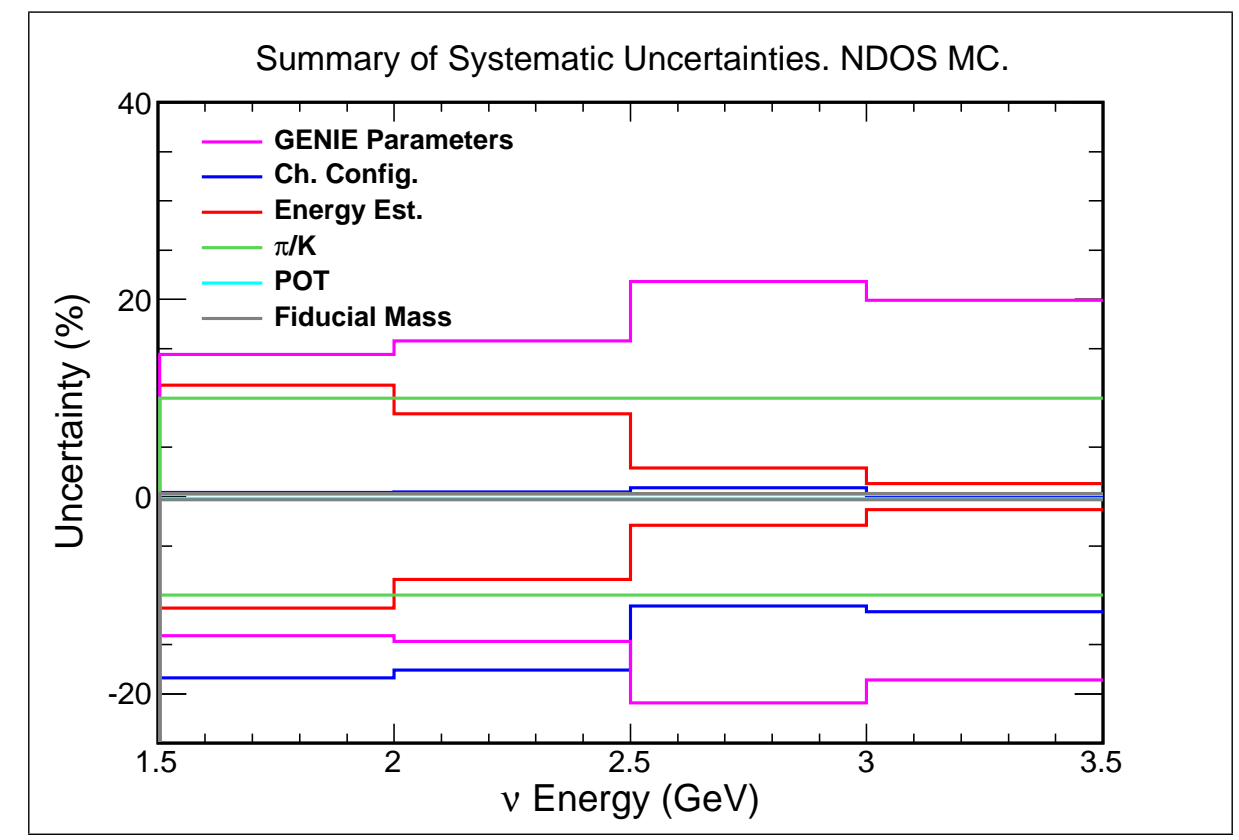

Figure 7.9 Summary Of Systematic Uncertainties. The systematic uncertainties: $\delta G$ (magenta), $\delta C$ (dark blue), $\delta E$ (red), $\delta R$ (green), $\delta P$ (light blue), and $\delta m$ (grey), are presented as a function of the neutrino energy.

The total systematic uncertainty associated to the number of events combines all the systematic uncertainties discussed in the previous sections. The systematic uncertainties presented in figure 7.3, 7.4, and 7.6, for energy estimation $(\delta E)$, channels configuration $(\delta C)$, and GENIE parameters $(\delta G)$, respectively, are combined along with the systematic 


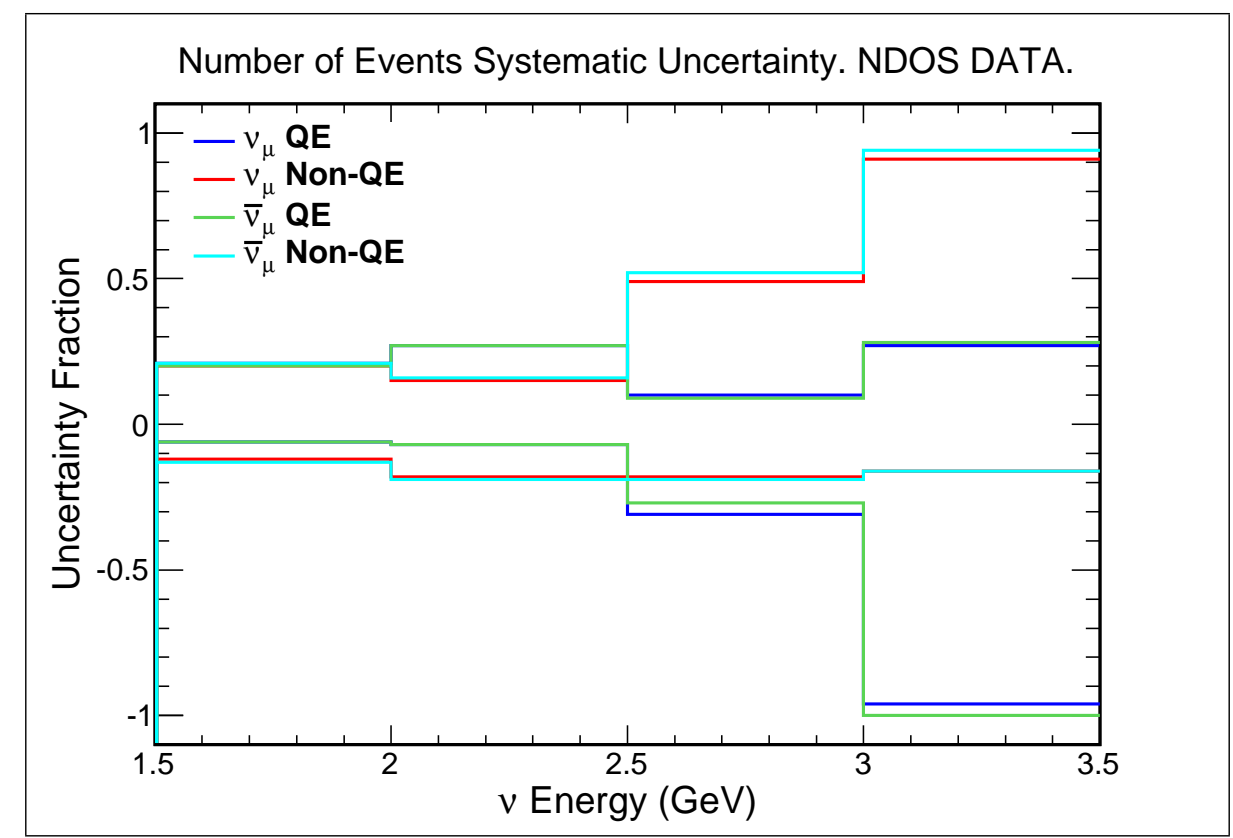

Figure 7.10 Systematic Uncertainty On The Number Of Events As A Function Of The Neutrino Energy. Each neutrino kind and interaction type is represented by a color.

uncertainty associated to the POT $(\delta P)$, the fiducial mass $(\delta m)$, and the charged kaons to pions ratio $(\delta R)$. All these uncertainties are dimensionless, and represent the uncertainty in the number of events due to each of the variables mentioned above. The correlations between all these uncertainties are of the order of the effect themselves, hence these second order effects are ignored, and the uncertainties are considered independent of each other, therefore these can be combined in quadrature:

$$
\delta \mathcal{N}=\sqrt{\delta E^{2}+\delta C^{2}+\delta G^{2}+\delta P^{2}+\delta m^{2}+\delta R^{2}} .
$$

The summary of all these uncertainties, which contribute to obtain the uncertainty in the number of selected events $(\delta \mathcal{N})$ before the unfolding procedure is applied, is shown in figure 7.9. There are four equations (7.2) associated with each energy bin, one for each interaction type and neutrino type: $\nu_{\mu} \mathrm{QE}, \nu_{\mu}$ non-QE, $\bar{\nu}_{\mu} \mathrm{QE}$, and $\bar{\nu}_{\mu}$ non-QE. 


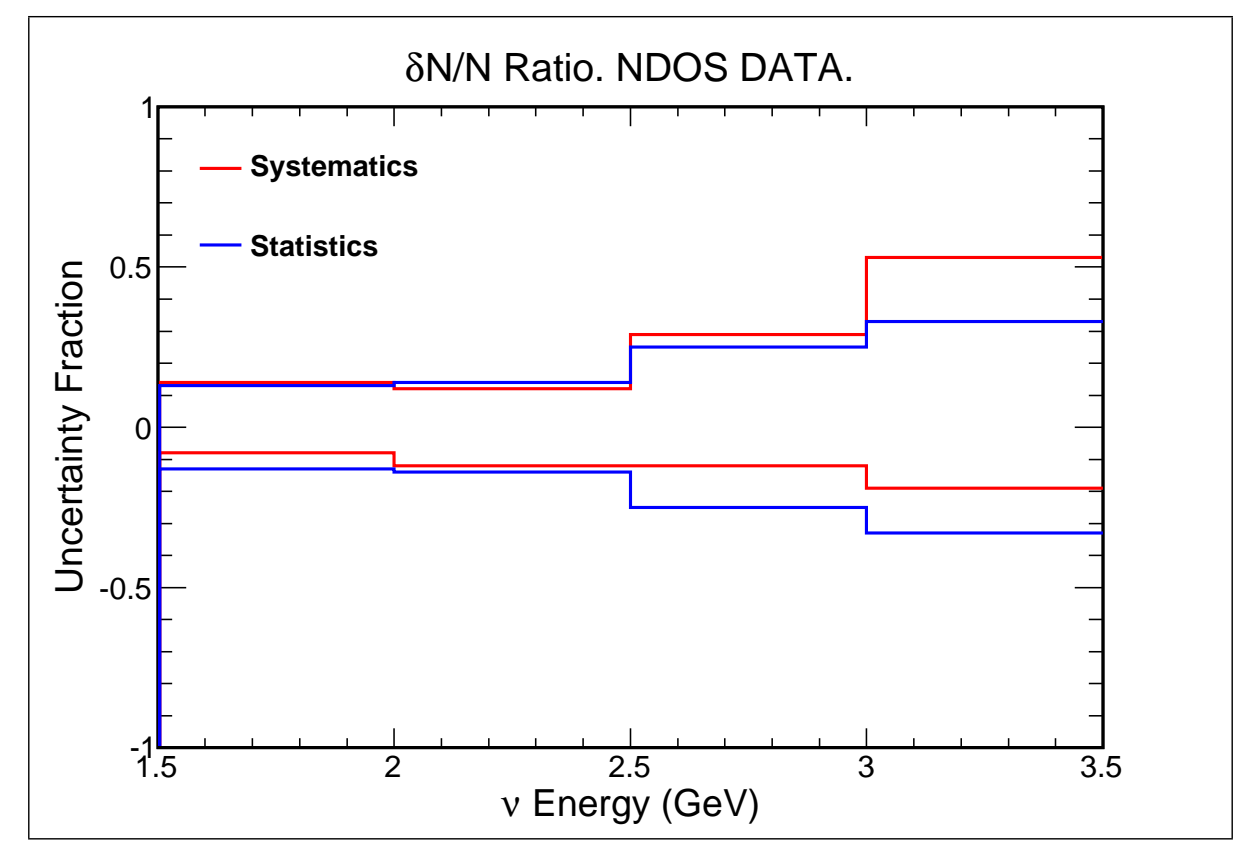

Figure $7.11 \delta N / N$ As A Function Of The Neutrino Energy. Ratios between the systematic uncertainty and the measured number of events (red), and the statistical uncertainty and the measured number of events (blue).

The combination of all the systematic uncertainties given by equation 7.2 are presented in figure 7.10. Once equation 7.2 is computed, the unfolding algorithm is applied, and a new unfolded uncertainty in the number of selected events $\left(\delta \mathcal{N}^{\prime}\right)$ is generated. The total systematic uncertainty on the unfolded number of events $(\delta N)$ is the combination in quadrature of $\delta \mathcal{N}^{\prime}$ and $\delta U$ :

$$
\delta N=\sqrt{\delta \mathcal{N}^{\prime 2}+\delta U^{2}} .
$$

The ratios between the total systematic uncertainties, $\delta N$, and the measured number of events, and the statistical uncertainties and the measured number of events, are compared in figure 7.11. Statistical and systematic uncertainties are of the same order, about $12 \%$, in the region of the charged kaons peak in the neutrino energy, i.e. from $1.5 \mathrm{GeV}$ to $2.5 \mathrm{GeV}$. 


\section{Chapter 8}

\section{Analysis}

The event selection criteria presented in chapter 5, and the energy reconstruction process presented in chapter 6 , are applied to the NDOS data in order to find the observed number of $\nu_{\mu}+\bar{\nu}_{\mu} \mathrm{CC}$ events.

\subsection{Data And Monte Carlo Simulation Comparison}

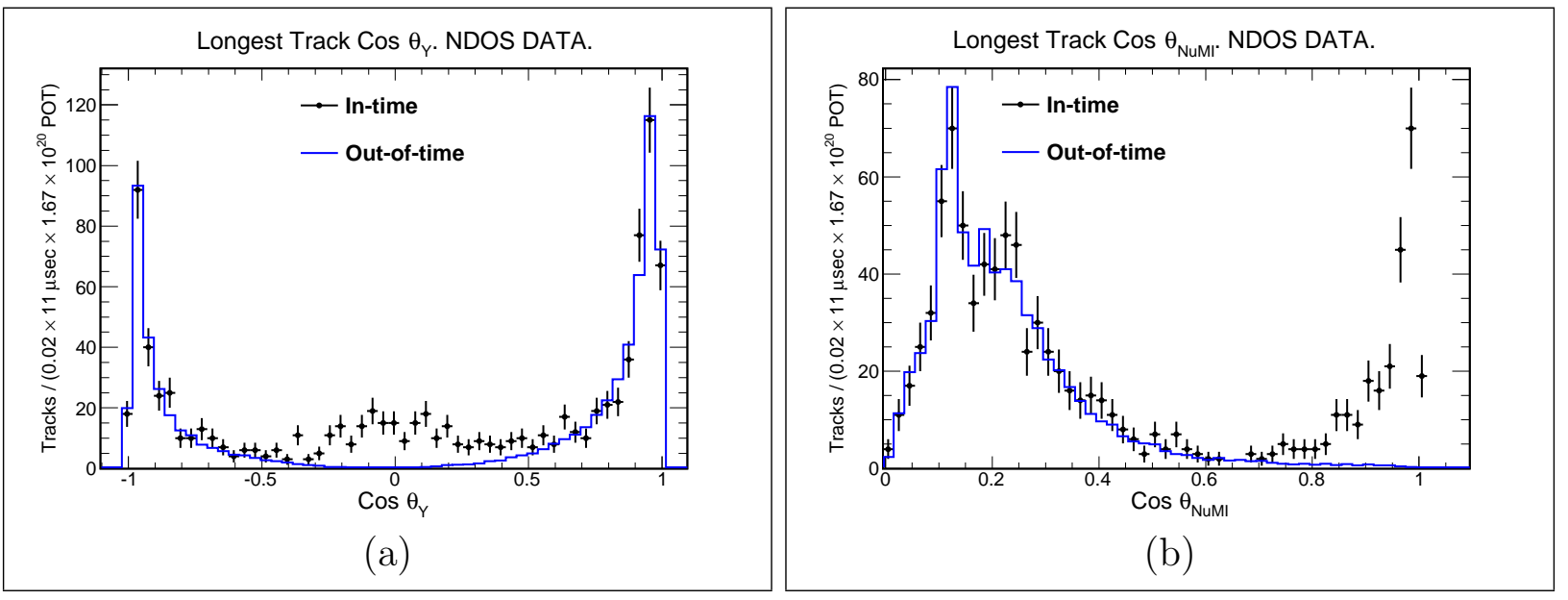

Figure 8.1 Angles Of The Longest Track. (a) Longest track's $\cos \theta_{Y}$ for out-of-time cosmic data (blue), and in-time data (black). (b) Longest track's $\cos \theta_{\mathrm{NuMI}}$.

NDOS data are separated (see section 4.4) into those recorded in-time (within the $11 \mu \mathrm{s}$ NuMI time window), where mixtures of neutrino interactions and cosmic rays are expected, and out-of-time, where only cosmic rays are expected, as shown in figure 8.1. The cosmic ray background expected in the in-time window is the out-of-time data normalized by the 
ratio of the time windows widths, given by:

$$
\text { Cosmic } \rightarrow \text { Cosmic } \times \frac{11}{429}
$$

The regions where cosmic rays are the dominant contribution are: $\left|\cos \theta_{Y}\right|>0.6$, and $\cos \theta_{\text {NuMI }}<0.6$. The in-time distributions without a cosmic ray background, obtained by subtracting the out-of-time data, can be compared with the distributions predicted by the MC simulation, as shown in figures $8.2 \mathrm{a}$ and $8.2 \mathrm{~b}$. In these figures, all the cuts presented in chapter 5 (see equations $(5.1,5.4,5.8$, and 5.13)) are applied, except the angular cuts. The MC simulation distributions are normalized to the data POT such that:

$$
\mathrm{MC} \rightarrow \mathrm{MC} \times \frac{\mathrm{POT}_{\text {Data }}}{\mathrm{POT}_{\mathrm{MC}}}=\mathrm{MC} \times \frac{1.67386 \times 10^{20}}{7.32109 \times 10^{21}}
$$

The background subtracted data has the general characteristics predicted by the MC simulation with the $\cos \theta_{Y}$ distribution peaked near zero, and the $\cos \theta_{\mathrm{NuMI}}$ distribution strongly peaked near 1 . The only significant deviation is that the number of events predicted by the MC simulation is higher than is seen in data, which is evident in the reduced height of the peaks at: 1 in the $\cos \theta_{\mathrm{NuMI}}$, and 0 in the $\cos \theta_{Y}$ distributions. When the shapes of the distributions are compared, these are in good agreement, as shown in figures 8.2c and 8.2d. Figure 8.2 illustrates the effect of eliminating the out-of-time data background. However, in the analysis, the cosmic background subtraction of the data is done at the number of events level (see figure 8.6). In order to verify that the subtraction of out-of-time data from in-time data was adequate, five random $11 \mu$ s time windows from the out-of-time data sample are chosen and treated as in-time data. The remaining out-of-time data are 


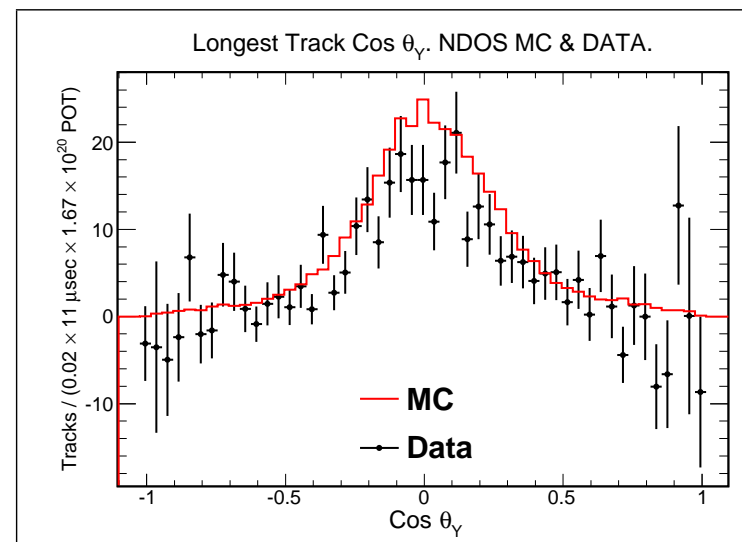

(a)

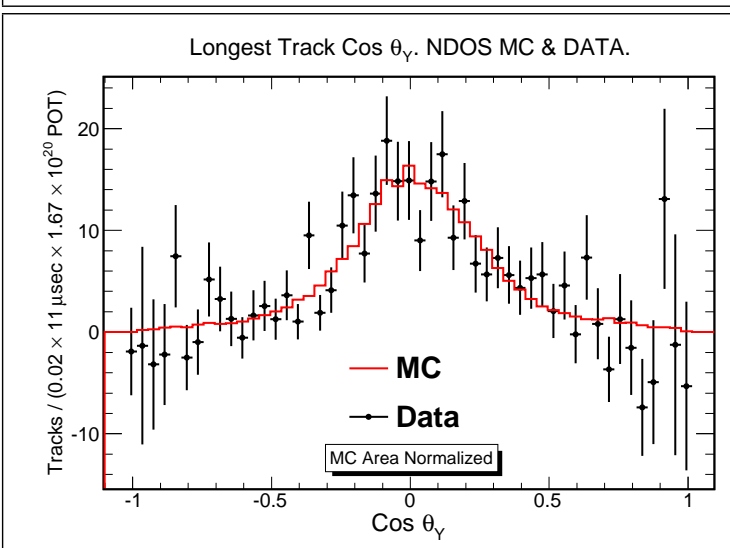

(c)

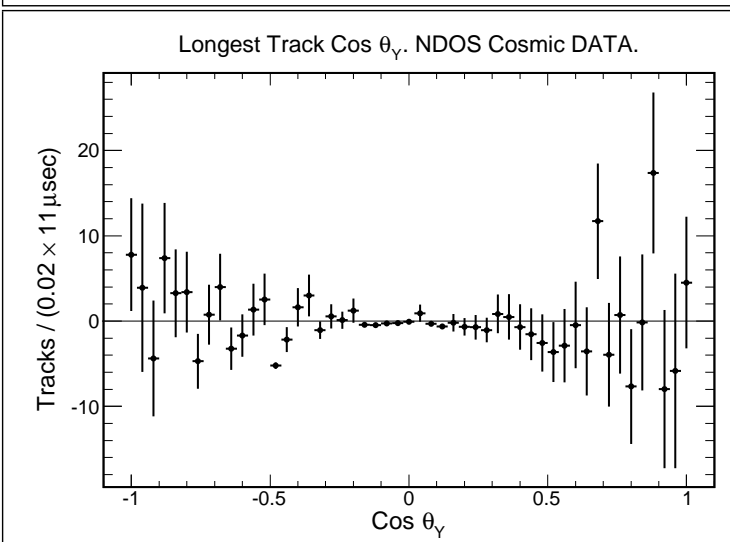

(e)

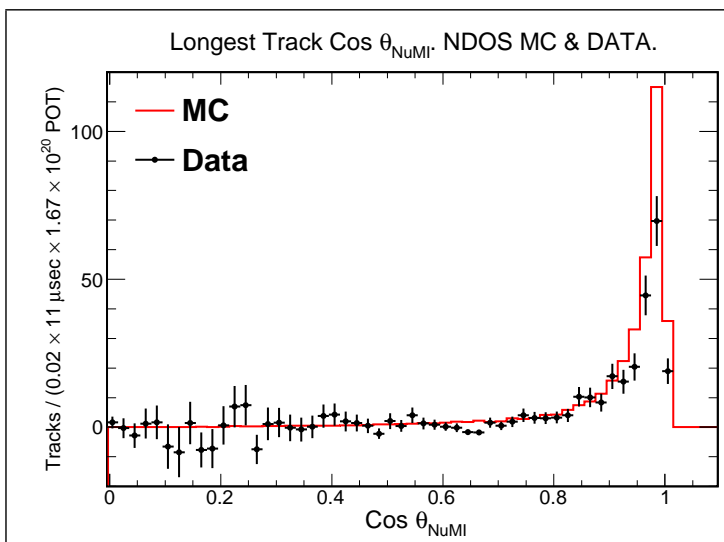

(b)

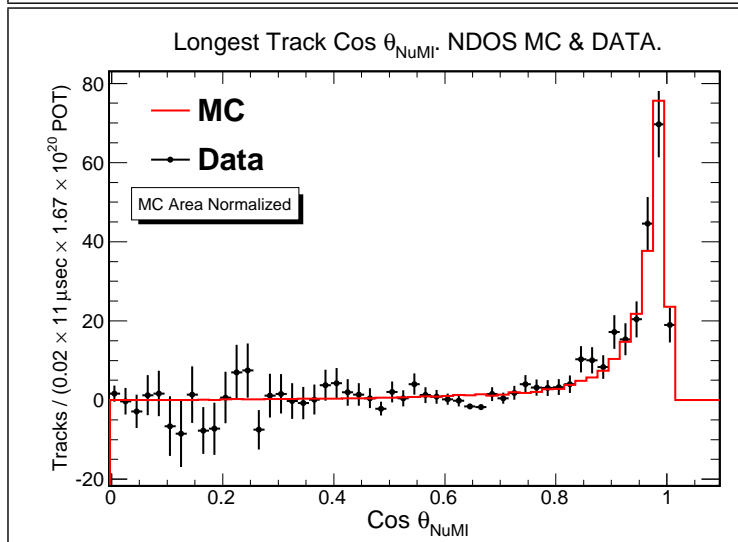

(d)

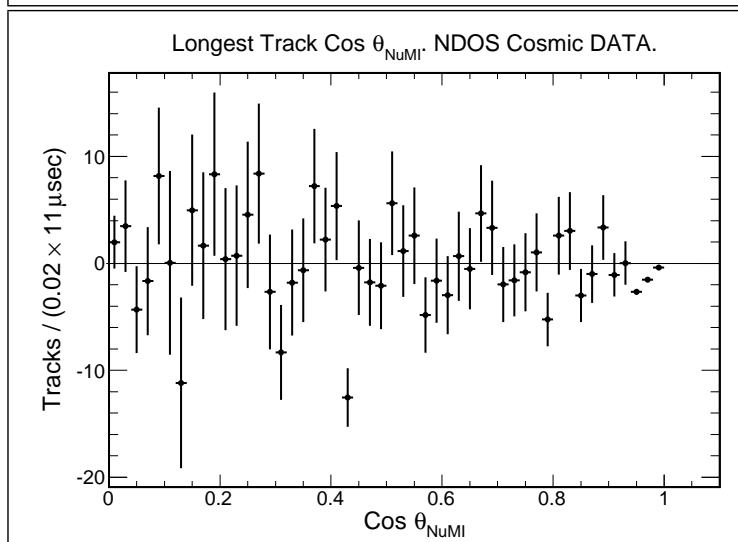

(f)

Figure 8.2 Longest Track Angular Distributions Without Cosmic Background. The angular distributions for the longest track with a subtraction of the cosmic ray background determined from out-of-time data. Longest track's $\cos \theta_{Y}$ MC simulation (red) and data (black) (a) POT normalized and (c) MC simulation area normalized to data. Longest track's $\cos \theta_{\text {NuMI }}$ (b) POT normalized and (d) MC simulation area normalized to data. Data means: in-time data minus out-of-time data. Longest track's (e) $\cos \theta_{Y}$ and (f) $\cos \theta_{\mathrm{NuMI}}$, for fake in-time data minus normalized out-of-time data. 
normalized as indicated in equation (8.1), however this time the factor of 429 is replaced by a factor of 418 to take into account that now two, and not just one, $11 \mu$ s time windows are subtracted from the full $440 \mu$ s time window. The out-of-time data are subtracted from the fake in-time data, and the results are consistent with zero, as seen in figures 8.2e and 8.2f. Each of the five fake in-time data samples chosen from the out-of-time data present similar behaviors to the ones seen in these two figures. The deviations from zero seen in figure 8.2a, for $\left|\cos \theta_{Y}\right|>0.6$, and in figure $8.2 \mathrm{~b}$, for $\cos \theta_{\mathrm{NuMI}}<0.5$, are consistent with the deviations (of statistical origin) seen in figure 8.2e and 8.2f, respectively, for the same intervals. These fluctuations occur in the regions where most of the cosmic data exists, however, the data in these regions is rejected by the cosmic cuts presented in equation (5.11), hence, these do not affect the analysis.
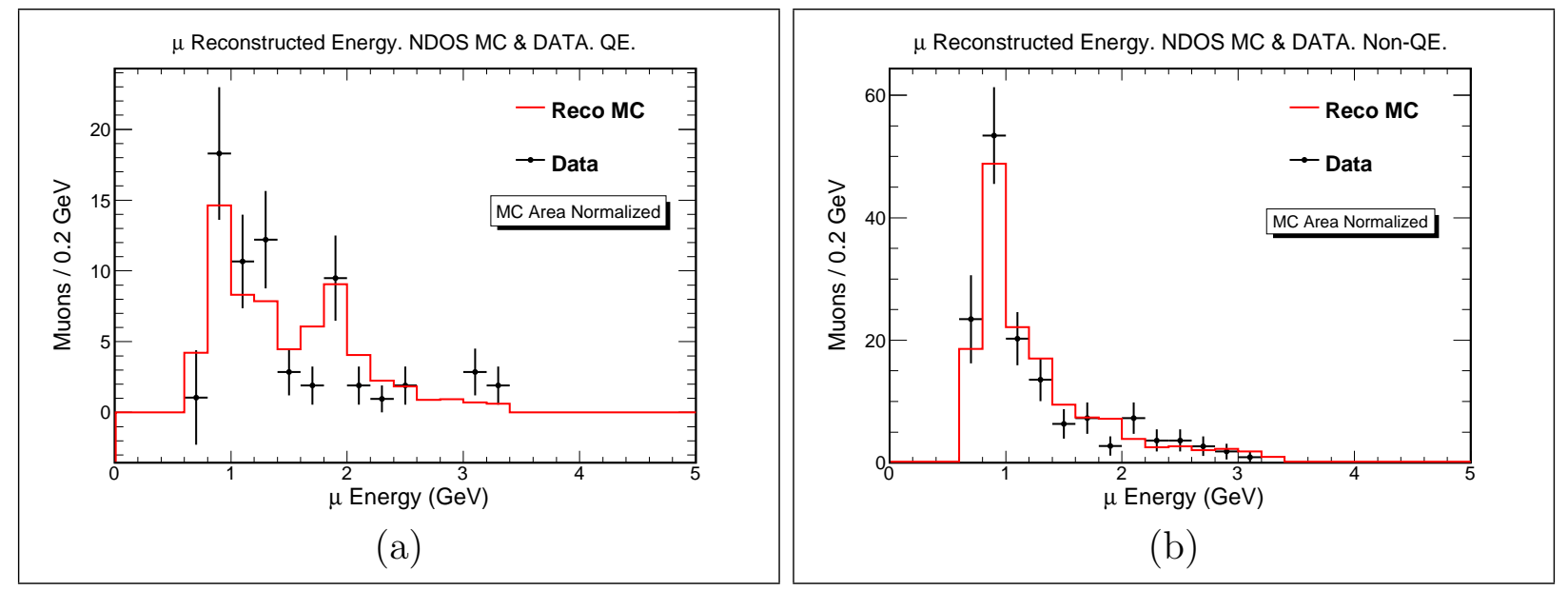

Figure 8.3 Reconstructed Muon Energy Distributions, No Cosmic Background. Reconstructed muon energy in the MC simulation (red) and in-time data (black) for (a) QE and (b) non-QE interactions with a subtraction of the cosmic ray background determined from out-of-time data. MC simulation area normalized to data.

The neutrino energy is reconstructed by adding the calorimetric energy and the muon energy from range. These individual components in the data are compared to the MC simulation, however, given the discrepancy in normalization between the MC simulation and 
the recorded data described above, only shape comparisons of the individual components are made. In addition, a cosmic background subtraction has been applied. The MC simulation provides a good representation for the shape of the muon energy distribution in the non-QE sample, as shown in figure 8.3b. The QE distributions require a deeper analysis because there are several data points below the MC near $2 \mathrm{GeV}$. A Kolmogorov-Smirnov test [112] is done in order to verify if the prediction of the MC simulation agrees with the data. The test results give a maximum deviation $(D)$ of: $D=0.0233$, a Kolmogorov-Smirnov test statistic $(K S)$ of: $K S=0.628$, and a p-value of: $\mathrm{p}=0.825$. Based on these results, the two QE distributions are in good agreement. A p-value greater than 0.1 implies that there is a low probability for the null hypothesis to be false. In this case, the null hypothesis is that the two cumulative distribution functions (CDF) in figure 8.4 are drawn from the same distribution, i.e. these represent the same physics. A CDF describes the probability that a random variable (sample value) $R$, with a given probability distribution $F(R)$, will be found to have a value less than or equal to $r$ [112].

The QE and non-QE distributions in figure 8.3 share the peak around $1 \mathrm{GeV}$. The muon energy distribution for QE interactions exhibits another peak in energy just below $2 \mathrm{GeV}$. This reflects the maximum in the neutrino flux at $2 \mathrm{GeV}$ due to charged kaon decays, and the general lack of energy in the hadronic system of a QE neutrino interaction. These $2 \mathrm{GeV}$ muons are likely to be contained only if these have $\cos \theta_{\mathrm{NuMI}} \approx 1$, otherwise these are likely to leave the detector. There are more contained $2 \mathrm{GeV}$ muons in the QE sample because these are likely to keep the direction of the neutrino, therefore traveling towards the muon catcher. Non-QE events have higher $y$, hence their muons are, in general, less energetic.

The simulated hadronic energy distribution in the non-QE sample is clearly consistent with the recorded data, as shown in figure 8.5. This will enable a reliable neutrino energy 


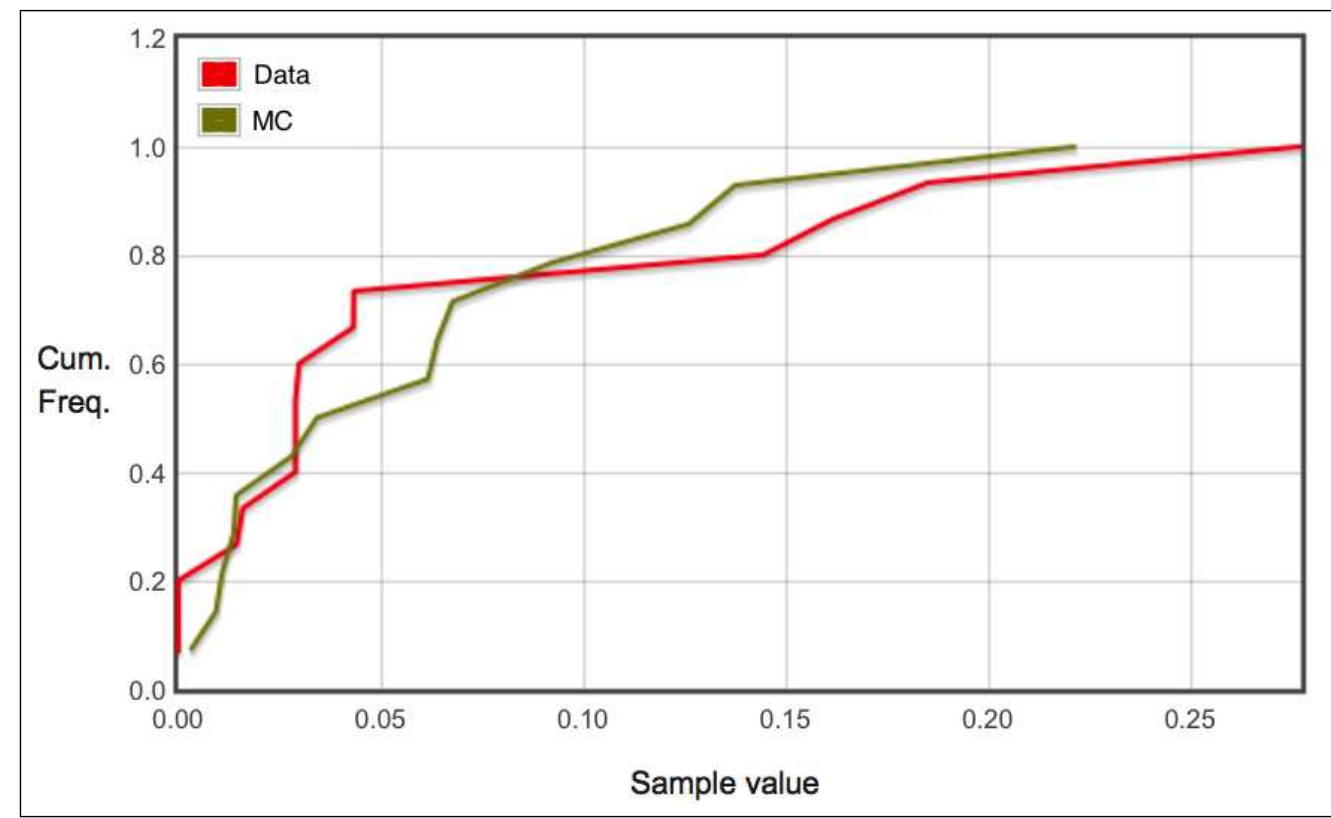

Figure 8.4 Cumulative Distribution Functions For Muons. Data (red) and MC simulation (green).

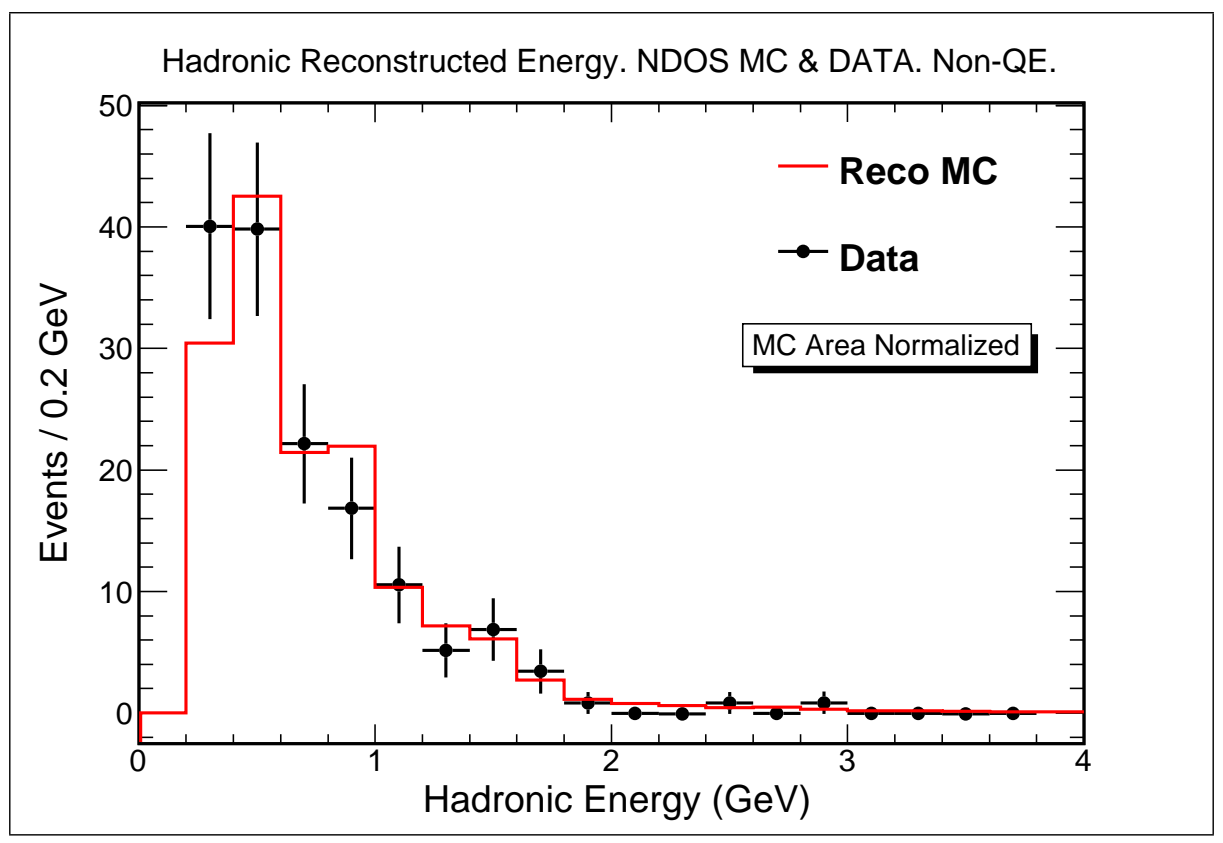

Figure 8.5 Hadronic Energy Distributions, No Cosmic Background. Reconstructed MC simulation (red) and in-time data (black) non-QE energy distributions. MC simulation area normalized to data. Cosmic background subtracted. 
calculation using the sum of the muon and hadronic energies. The mean hadronic energy is around $0.7 \mathrm{GeV}$, which is about half of the mean energy of the muons.

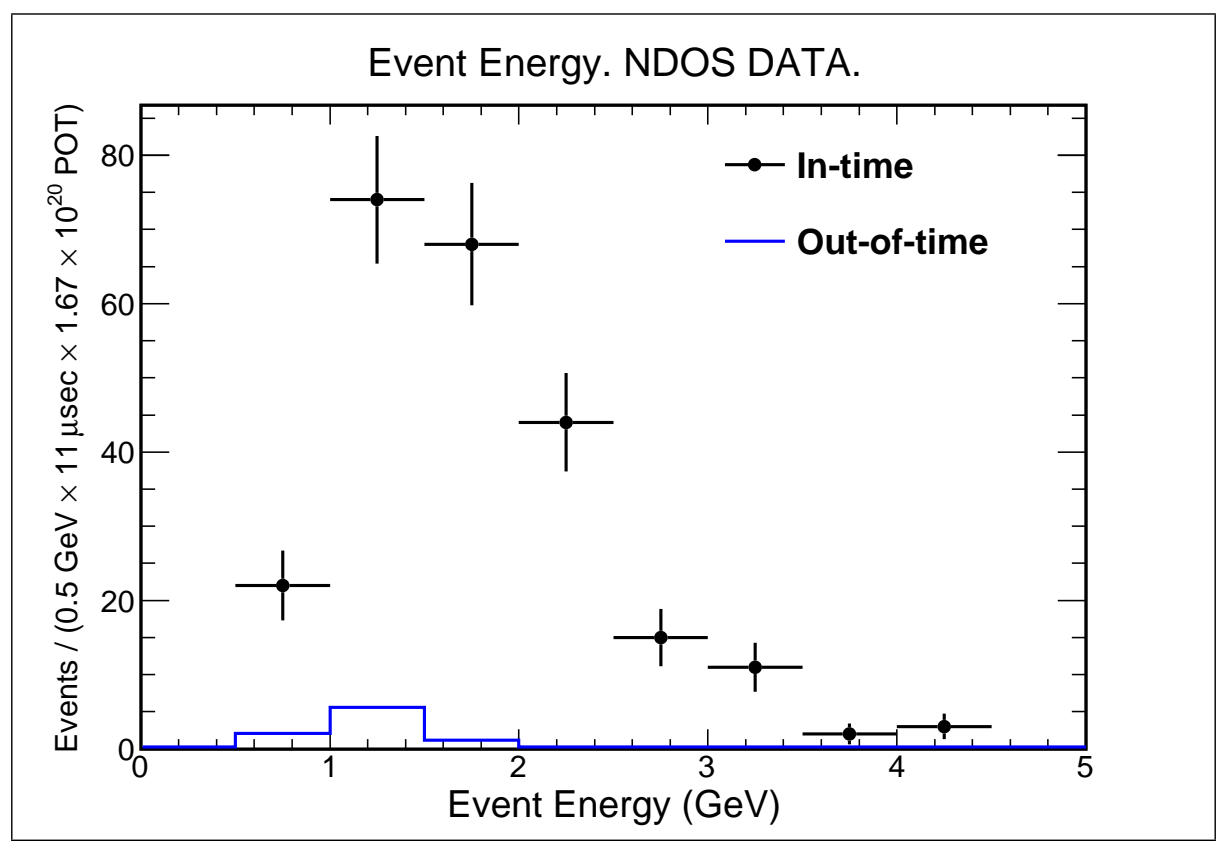

Figure 8.6 Selected Candidate Events Energy Distributions. In-time (black) and out-of-time (blue) data energy distributions with cosmic cuts applied.

The level of the cosmic ray background remaining in the in-time data is obtained by applying all cuts to the out-of-time data. Near $1 \mathrm{GeV}$ this cosmic ray background contribution is approximately $10 \%$ of the number of events seen in the in-time data, as shown in figure 8.6. The consequences of the cuts, presented in table 5.1 and in equation (5.11) on the data sample are summarized in table 8.1. $239 \nu_{\mu}+\bar{\nu}_{\mu}$ CC candidate events are selected. The cosmic background subtraction is done to this set of selected candidate events.

The number of selected QE candidate events $\left(M_{q}\right)$ is the sum of a number of true QE events $\left(T_{q}\right)$ and a number of true non-QE events, or fake QE events $\left(F_{q}\right)$, as seen in the first row of the matrix in figure 8.7. Similarly, the number of selected non-QE candidate events $\left(M_{n}\right)$ is the sum of the rest of the true non-QE events $\left(T_{n}\right)$ and the rest of the true $\mathrm{QE}$ 


\begin{tabular}{|c|c|}
\hline Cut Name & Number of Events \\
\hline \hline All events (no cut) & 146085 \\
\hline 3D & 93722 \\
\hline LTL & 54986 \\
\hline VR & 3715 \\
\hline Containment & 993 \\
\hline MIP & 939 \\
\hline Cosmic & 239 \\
\hline
\end{tabular}

Table 8.1 Event Selection Cuts. Summary of the effect of all the event selection cuts on the data sample. The number of events represents the number of selected neutrino candidate events that passed each cut. Cosmic background subtraction is applied to the events set that passed all these cuts.

\begin{tabular}{|c|c|c|c|c|c|c|c|c|}
\hline$(\mathrm{p}, \epsilon) \backslash \mathrm{E}(\mathrm{GeV})$ & $0.5-1.0$ & $1.0-1.5$ & $1.5-2.0$ & $2.0-2.5$ & $2.5-3.0$ & $3.0-3.5$ & $3.5-4.0$ & $4.0-10.0$ \\
\hline \hline QE & & & & & & & & \\
Purity & $97.4 \%$ & $89.1 \%$ & $86.2 \%$ & $84.0 \%$ & $91.2 \%$ & $87.4 \%$ & $50.0 \%$ & $85.7 \%$ \\
Error & $0.5 \%$ & $0.7 \%$ & $0.6 \%$ & $0.7 \%$ & $1.0 \%$ & $1.3 \%$ & $2.7 \%$ & $1.6 \%$ \\
Efficiency & $78.4 \%$ & $71.7 \%$ & $71.6 \%$ & $68.1 \%$ & $64.4 \%$ & $48.1 \%$ & $22.0 \%$ & $35.3 \%$ \\
Error & $1.2 \%$ & $0.9 \%$ & $0.7 \%$ & $0.8 \%$ & $1.6 \%$ & $2.0 \%$ & $2.3 \%$ & $2.2 \%$ \\
\hline Non-QE & & & & & & & & \\
Purity & $34.6 \%$ & $79.1 \%$ & $88.1 \%$ & $87.5 \%$ & $85.8 \%$ & $82.0 \%$ & $89.8 \%$ & $97.7 \%$ \\
Error & $1.4 \%$ & $0.8 \%$ & $0.5 \%$ & $0.6 \%$ & $1.2 \%$ & $1.5 \%$ & $1.7 \%$ & $0.7 \%$ \\
Efficiency & $84.5 \%$ & $92.4 \%$ & $94.8 \%$ & $94.5 \%$ & $97.2 \%$ & $97.2 \%$ & $96.9 \%$ & $99.8 \%$ \\
Error & $1.0 \%$ & $0.6 \%$ & $0.4 \%$ & $0.4 \%$ & $0.6 \%$ & $0.7 \%$ & $1.0 \%$ & $0.2 \%$ \\
\hline
\end{tabular}

Table 8.2 Quasi-elastic And Non-quasi-elastic Classification Performance. Purities and efficiencies for QE and non-QE sample. The columns represent neutrino energy bins. 


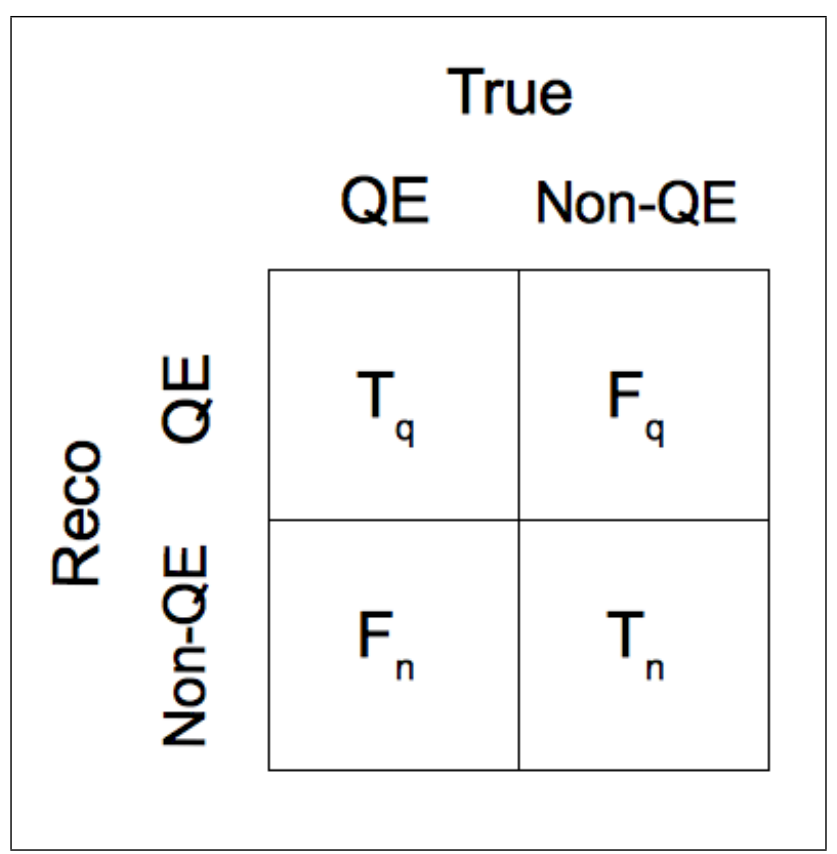

Figure 8.7 Efficiency And Purity Matrix For The QE And Non-QE Samples. This matrix aids to construct the efficiencies and purities related to the selection of reconstructed $\mathrm{QE}$ and non-QE candidate events.

events, or fake non-QE events $\left(F_{n}\right)$, as seen in the second row of the matrix in figure 8.7:

$$
M_{q}=T_{q}+F_{q}, \quad M_{n}=T_{n}+F_{n}
$$

The efficiencies $\left(\epsilon_{q}\right.$ and $\left.\epsilon_{n}\right)$ and purities $\left(\mathrm{p}_{q}\right.$ and $\left.\mathrm{p}_{n}\right)$ for the selection of $\mathrm{QE}$ and non-QE candidate events are defined as:

$$
\begin{gathered}
\epsilon_{q}=\frac{T_{q}}{N_{q}}, \quad \epsilon_{n}=\frac{T_{n}}{N_{n}} \\
\mathrm{p}_{q}=\frac{T_{q}}{M_{q}}, \quad \mathrm{p}_{n}=\frac{T_{n}}{M_{n}}
\end{gathered}
$$


where the total number of real QE events $\left(N_{q}\right)$ is:

$$
N_{q}=T_{q}+F_{n}=M_{q} \mathrm{p}_{q}+M_{n}\left(1-\mathrm{p}_{n}\right)
$$

and the total number of real non-QE events $\left(N_{n}\right)$ is:

$$
N_{n}=T_{n}+F_{q}=M_{n} \mathrm{p}_{n}+M_{q}\left(1-\mathrm{p}_{q}\right)
$$

The purity (see equation (8.4)) of the QE sample is $(89.1 \pm 0.8) \%$, and $(84.4 \pm 0.4) \%$ for the non-QE sample. The efficiency (see equation (8.4)) of the QE sample is $(71.2 \pm 1.0) \%$, and $(94.5 \pm 0.3) \%$ for the non-QE sample. The QE and non-QE samples purity and efficiency per energy bins are summarized in table 8.2. Once the number of selected neutrino candidate events (from the NDOS data), for QE and non-QE, is measured, the efficiencies and purities in table 8.2 are used to obtain the real number of selected candidate events per interaction, and per energy bin.

Three sample MC simulated events to illustrate the kind of topology that the selection, presented in relation (6.6), allows in the QE sample are shown in figure 8.8. The event display in figure 8.8 a represents the following interaction:

$$
\nu_{\mu}(0.8 \mathrm{GeV} / \mathrm{c})+{ }^{12} \mathrm{C} \rightarrow \mu(0.7 \mathrm{GeV} / \mathrm{c})+p(0.2 \mathrm{GeV} / \mathrm{c})
$$

The proton does not leave the interaction cell, and the muon's track is about $3 \mathrm{~m}$ long. There is no track reconstruction shown in these figures, and the colors of the cell hits represent the amount of energy deposited, which follows the color code on the lower right histogram. The reconstructed neutrino energy for this event is: $1.15 \mathrm{GeV}$, and the reconstructed hadronic 


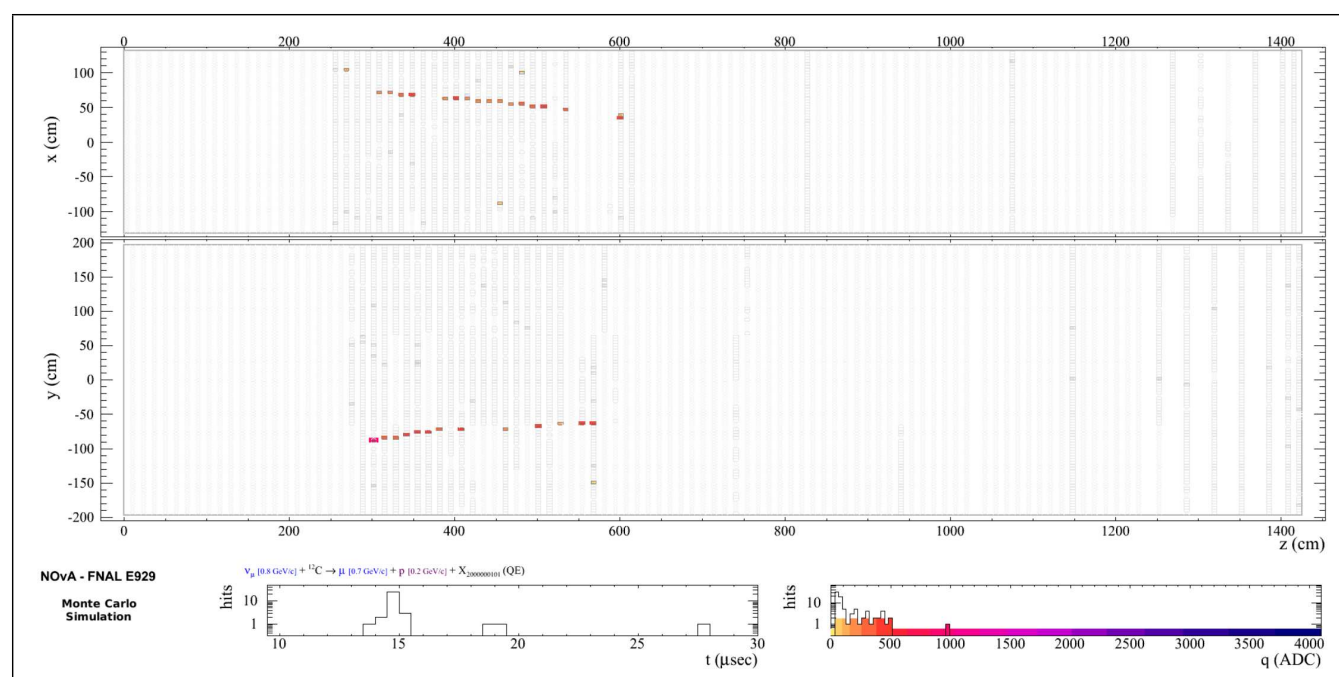

(a)

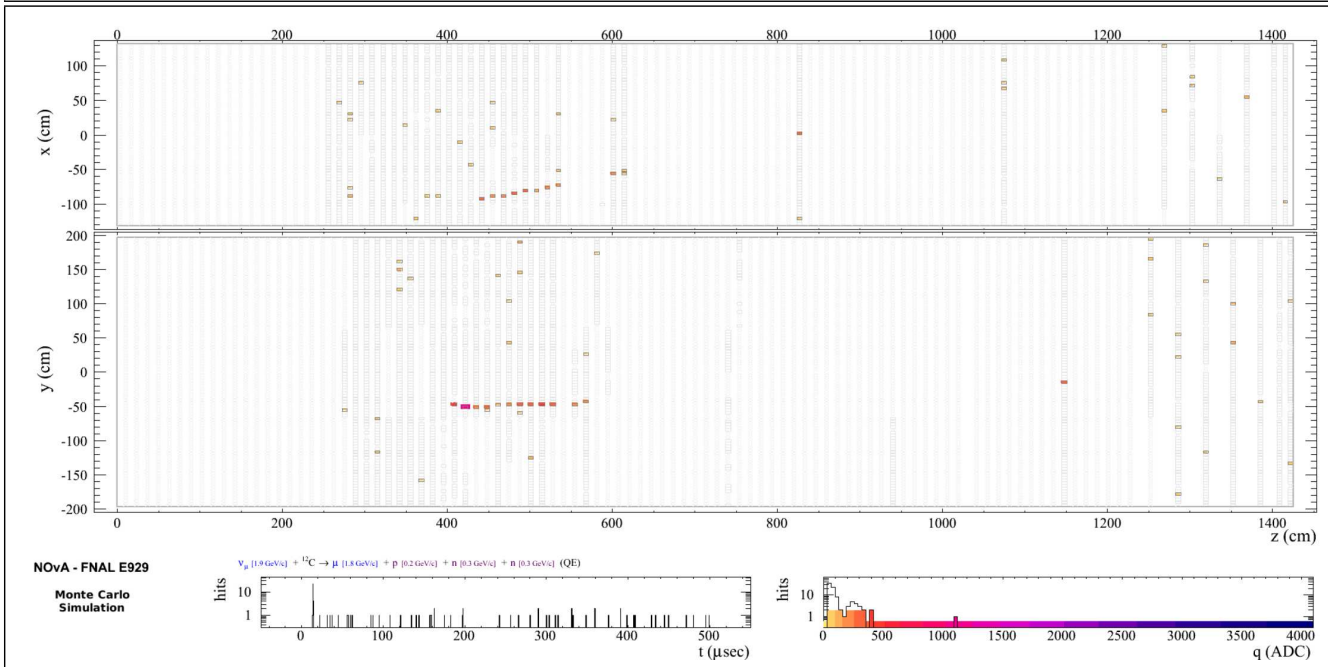

(b)

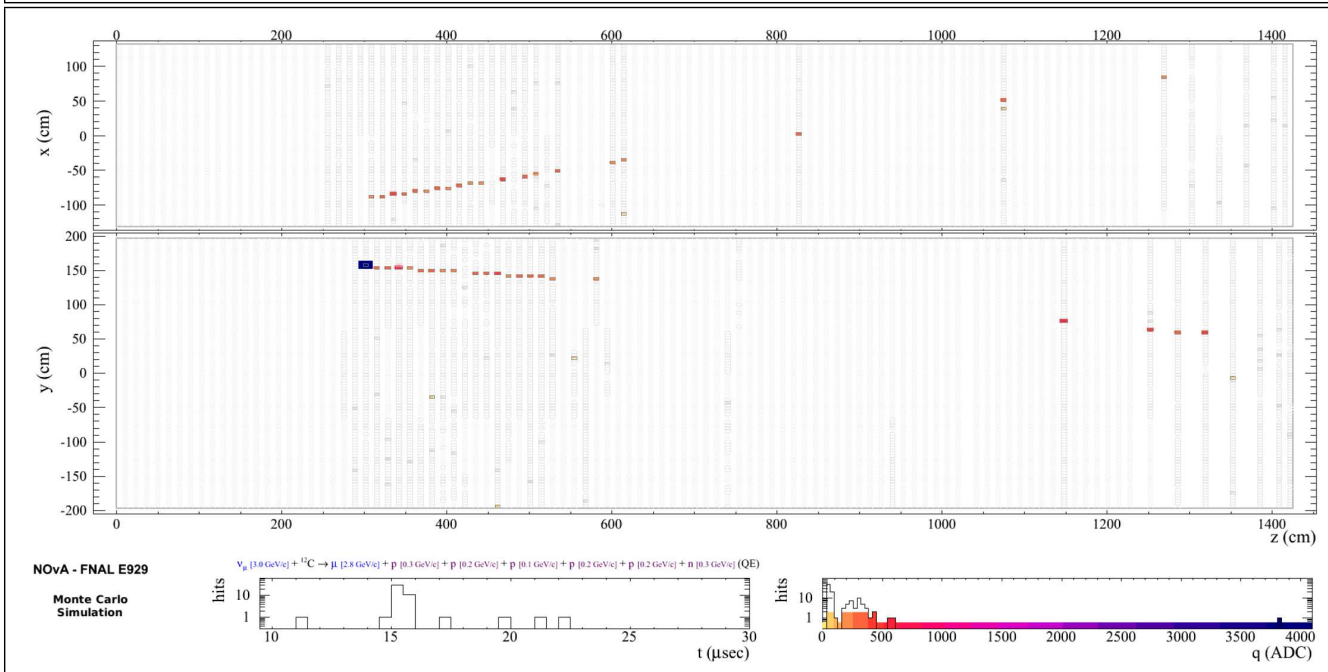

(c)

Figure 8.8 Sample Of Simulated QE Neutrino Events. 
energy is: $0.1 \mathrm{GeV}$. The interaction in the event display shown in figure $8.8 \mathrm{~b}$ is:

$$
\nu_{\mu}(1.9 \mathrm{GeV} / \mathrm{c})+{ }^{12} \mathrm{C} \rightarrow \mu(1.8 \mathrm{GeV} / \mathrm{c})+p(0.2 \mathrm{GeV} / \mathrm{c})+2 n(0.3 \mathrm{GeV} / \mathrm{c}) .
$$

The proton does not leave the interaction cell, and the muon's track is about $7 \mathrm{~m}$ long. The two neutrons do not deposit any reconstructable energy. This is the typical $\nu_{\mu} \mathrm{CC}$ event expected to be found in the NDOS data, at the $2 \mathrm{GeV}$ peak. The reconstructed neutrino energy for this event is: $1.90 \mathrm{GeV}$, and the reconstructed hadronic energy is: $0.1 \mathrm{GeV}$. The event display in figure 8.8c represents the following interaction:

$$
\nu_{\mu}(3.0 \mathrm{GeV} / \mathrm{c})+{ }^{12} \mathrm{C} \rightarrow \mu(2.8 \mathrm{GeV} / \mathrm{c})+4 p+n(0.3 \mathrm{GeV} / \mathrm{c})
$$

There are four protons, each with about $0.2 \mathrm{GeV} / \mathrm{c}$, that deposit all their energy in the interaction cell, and a neutron. The muon has $2.8 \mathrm{GeV} / \mathrm{c}$, which takes it half way into the muon catcher. Muons of these energies are only likely to be contained if these reach the muon catcher. The reconstructed neutrino energy for this event is: $2.85 \mathrm{GeV}$, and the reconstructed hadronic energy is: $0.1 \mathrm{GeV}$. The three sample MC simulated events are good representations of the QE sample, these deposit very little energy in cells that do not belong to the reconstructed track.

In contrast, three sample events selected by relation (6.6) illustrate the kind of topologies allowed in the non-QE sample. The event display in figure 8.9a represents the following interaction:

$$
\nu_{\mu}(2.0 \mathrm{GeV} / \mathrm{c})+p \rightarrow \mu(1.3 \mathrm{GeV} / \mathrm{c})+p(0.4 \mathrm{GeV} / \mathrm{c})+\pi^{+}(0.6 \mathrm{GeV} / \mathrm{c})
$$




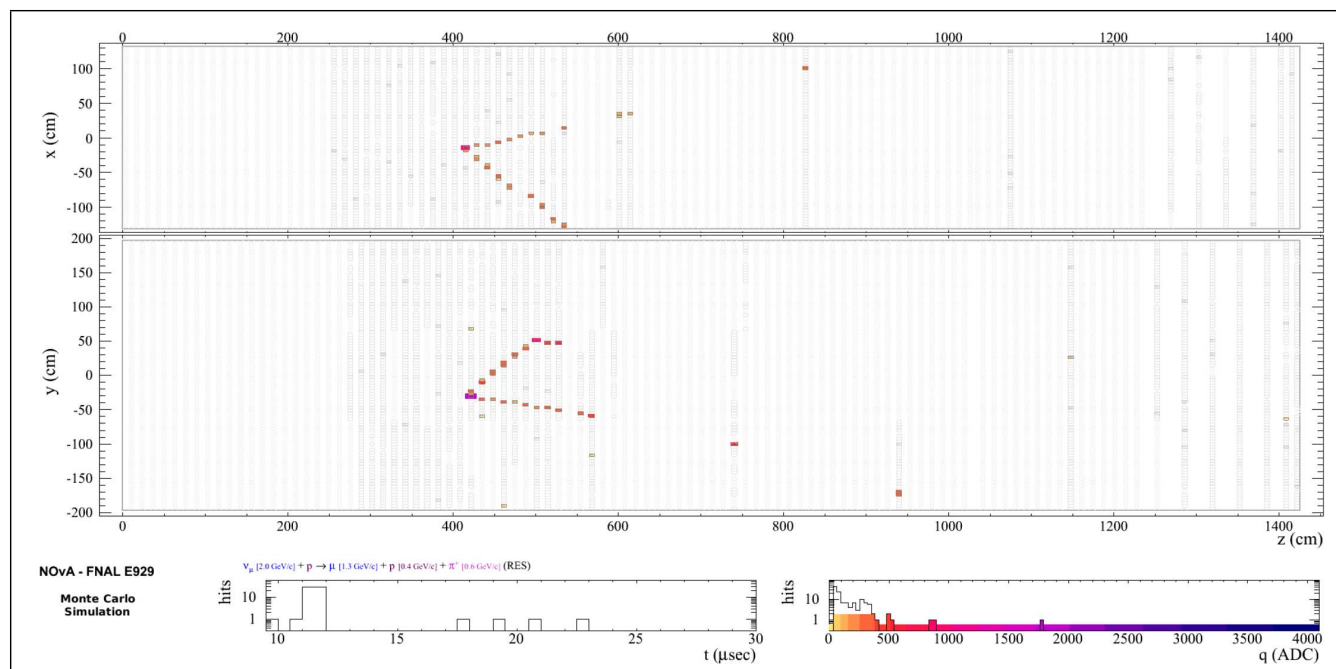

(a)

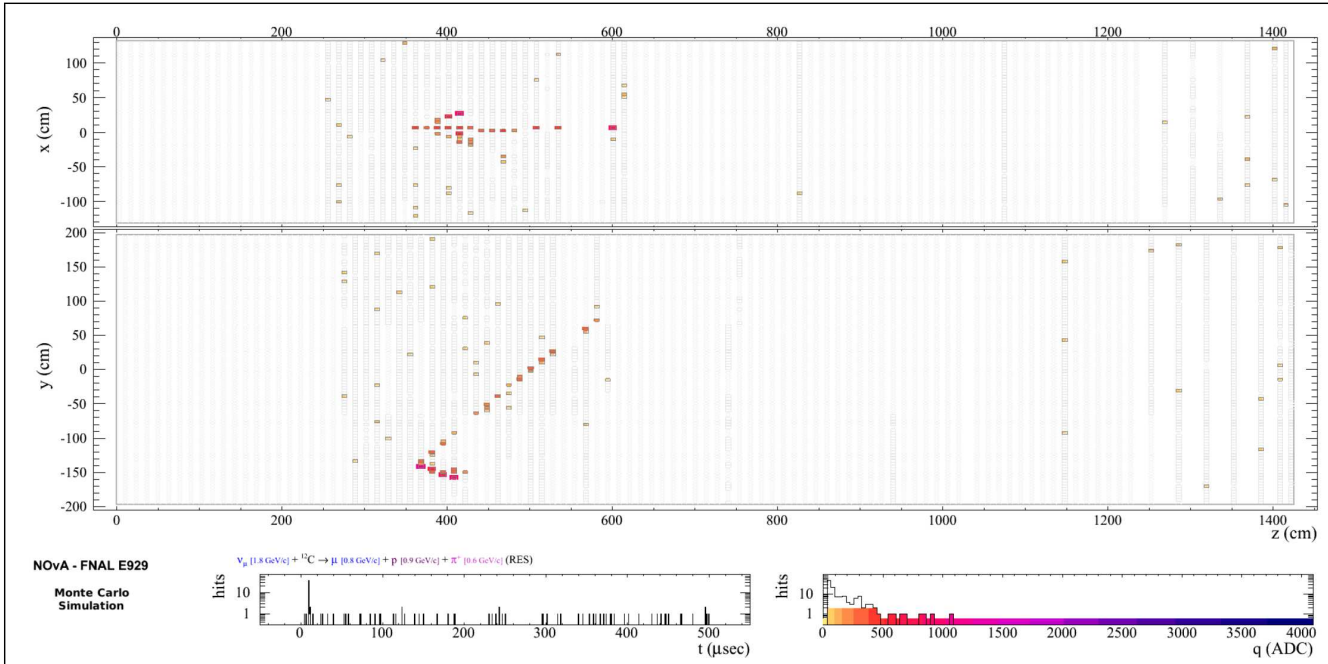

(b)

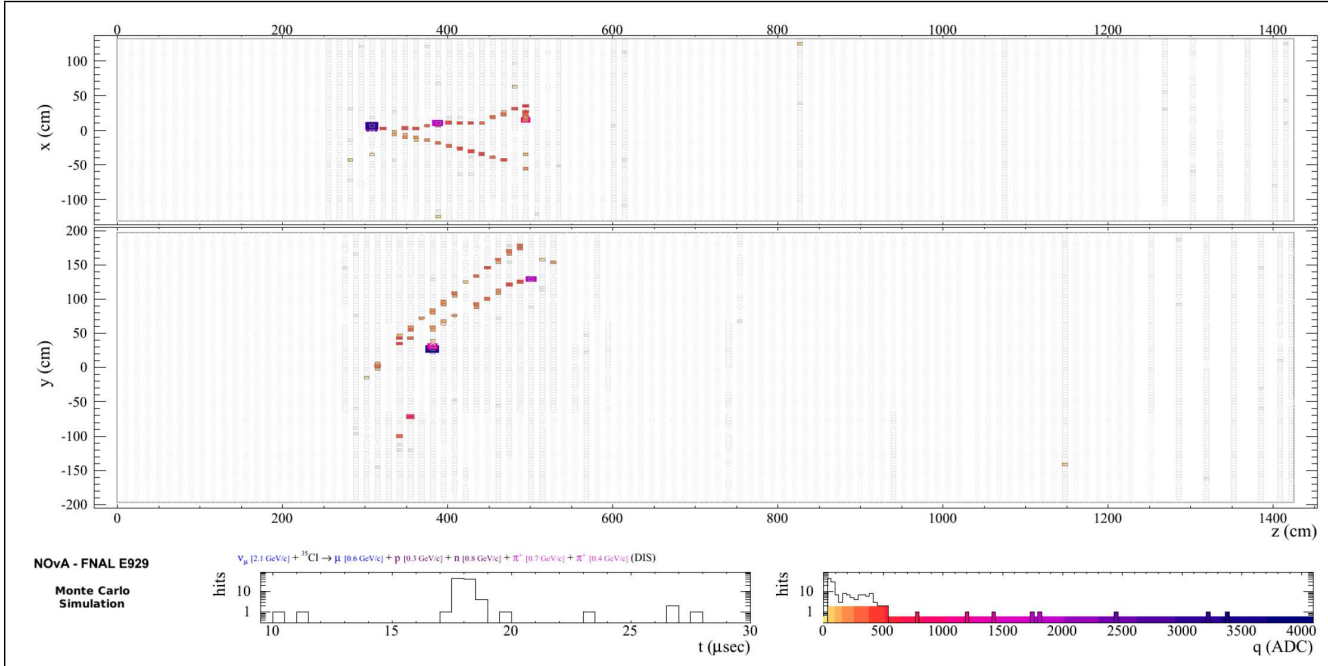

(c)

Figure 8.9 Sample Of Simulated Non-QE Neutrino Events. 
The proton does not leave the interaction cell, the muon's track is about $6 \mathrm{~m}$ long, and the $\pi^{+}$leaves a MIP-like track with a hard scattering at the end. This is a resonance event. The reconstructed neutrino energy for this event is: $2.15 \mathrm{GeV}$, and the reconstructed hadronic energy is: $0.9 \mathrm{GeV}$. The event display in figure $8.9 \mathrm{~b}$ represents the following interaction:

$$
\nu_{\mu}(1.8 \mathrm{GeV} / \mathrm{c})+{ }^{12} \mathrm{C} \rightarrow \mu(0.8 \mathrm{GeV} / \mathrm{c})+p(0.9 \mathrm{GeV} / \mathrm{c})+\pi^{+}(0.6 \mathrm{GeV} / \mathrm{c}) .
$$

Although this interaction is similar to the previous one, in this case the proton leaves a track with high energy depositions (top view, top track), and the $\pi^{+}$leaves a shower with some electromagnetic energy deposition associated with it (top view, bottom track). The muon's track is about $3 \mathrm{~m}$ long. This is a resonance event. The reconstructed neutrino energy for this event is: $1.95 \mathrm{GeV}$, and the reconstructed hadronic energy is: $0.8 \mathrm{GeV}$. The event display in figure $8.9 \mathrm{c}$ represents the following interaction:

$\nu_{\mu}(2.1 \mathrm{GeV} / \mathrm{c})+{ }^{35} \mathrm{Cl} \rightarrow \mu(0.6 \mathrm{GeV} / \mathrm{c})+p(0.3 \mathrm{GeV} / \mathrm{c})+n+\pi^{+}(0.7 \mathrm{GeV} / \mathrm{c})+\pi^{+}(0.4 \mathrm{GeV} / \mathrm{c})$.

The proton deposits most of its energy in the cell around: $Y=20 \mathrm{~cm}$ and $Z=375 \mathrm{~cm}$, the $0.7 \mathrm{GeV} / \mathrm{c} \pi^{+}$leaves a MIP-like track with a hard scattering at the end, the $0.4 \mathrm{GeV} / \mathrm{c}$ $\pi^{+}$leaves a scattered shower going downwards in the side view, the neutron deposits no reconstructable energy, and the muon's track is about $2 \mathrm{~m}$ long. This is a DIS event. The reconstructed neutrino energy for this event is: $2.3 \mathrm{GeV}$, and the reconstructed hadronic energy is: $1.55 \mathrm{GeV}$. The muons in the non-QE sample have energies around $1 \mathrm{GeV}$, and are likely to be contained.

A sample of selected QE neutrino candidates, similar to the one presented in figure 8.8, is 


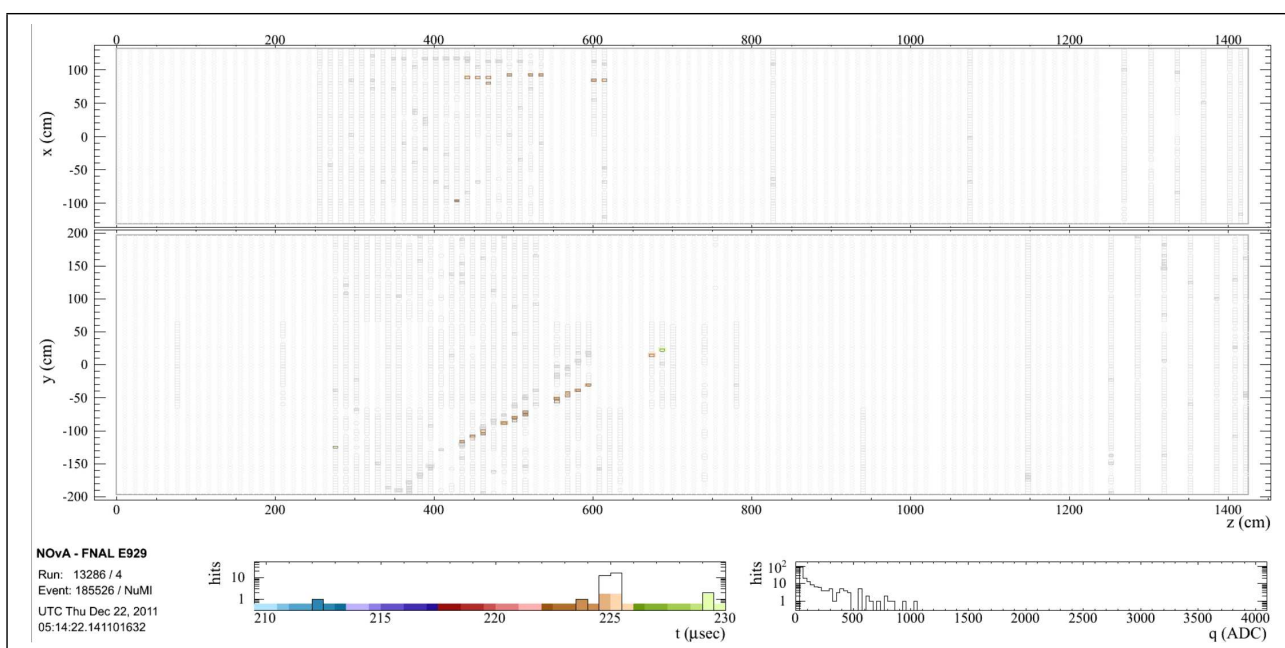

(a)

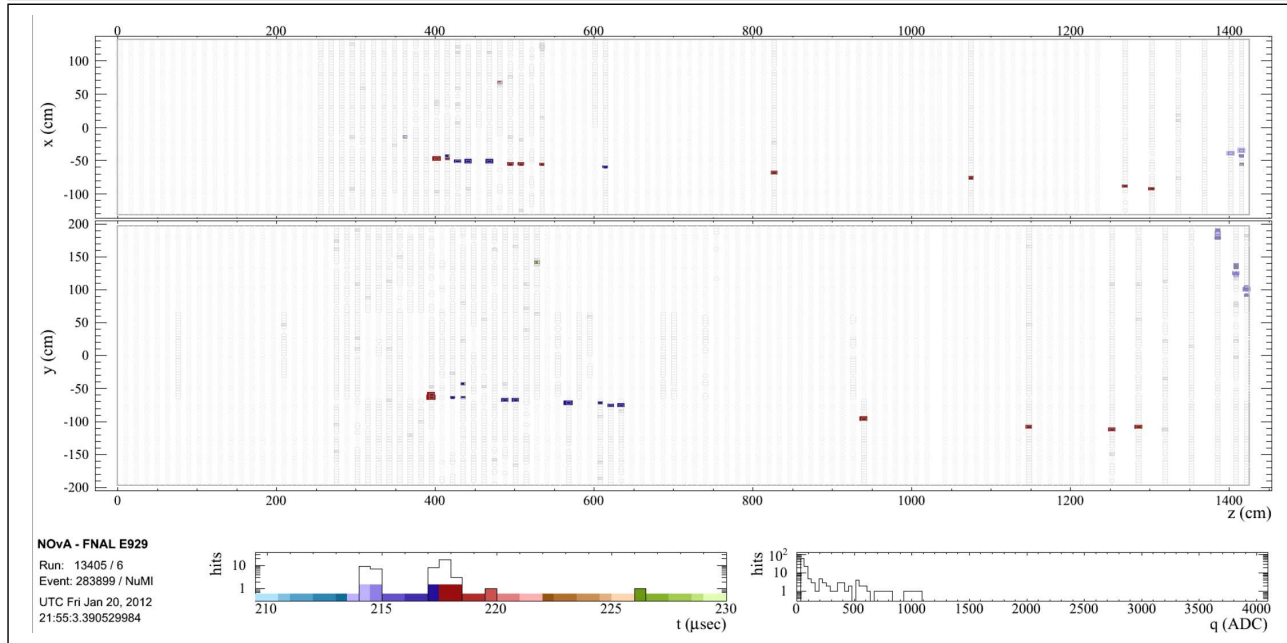

(b)

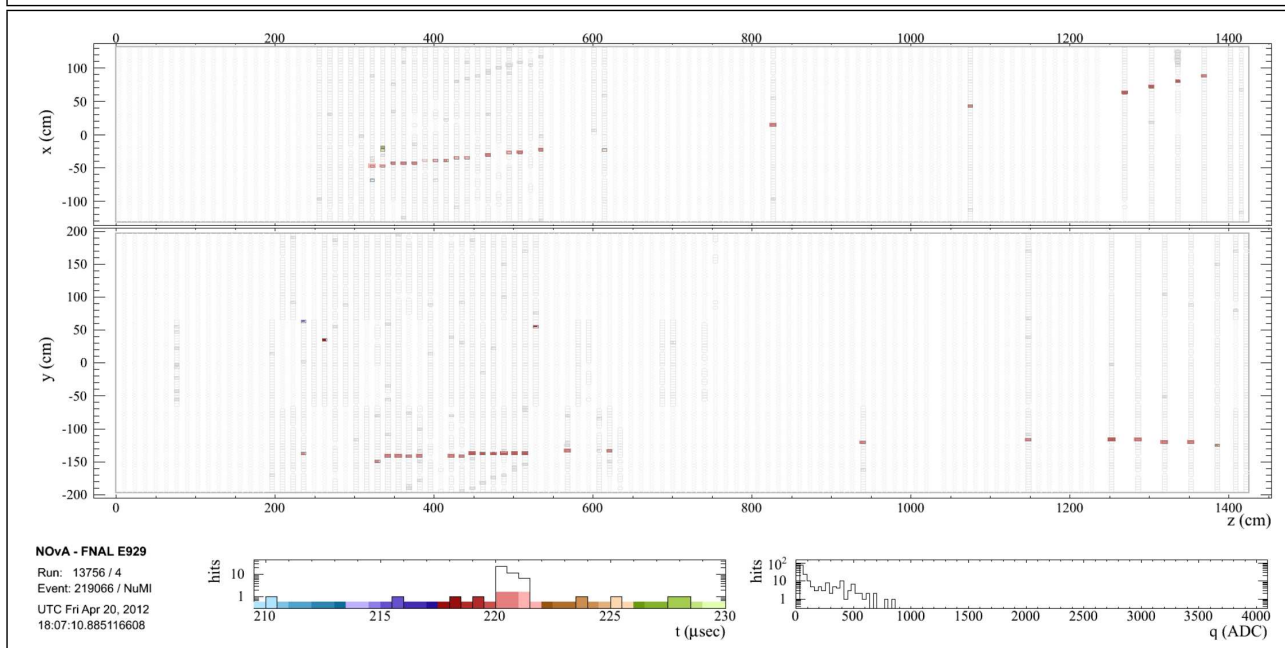

(c)

Figure 8.10 Sample Of QE Neutrino Candidates. (a) Selected neutrino candidate with $1.0 \mathrm{GeV}$ of estimated energy. (b) Selected neutrino candidate with $2.5 \mathrm{GeV}$ of estimated energy. (c) Selected neutrino candidate with $3.3 \mathrm{GeV}$ of estimated energy. 


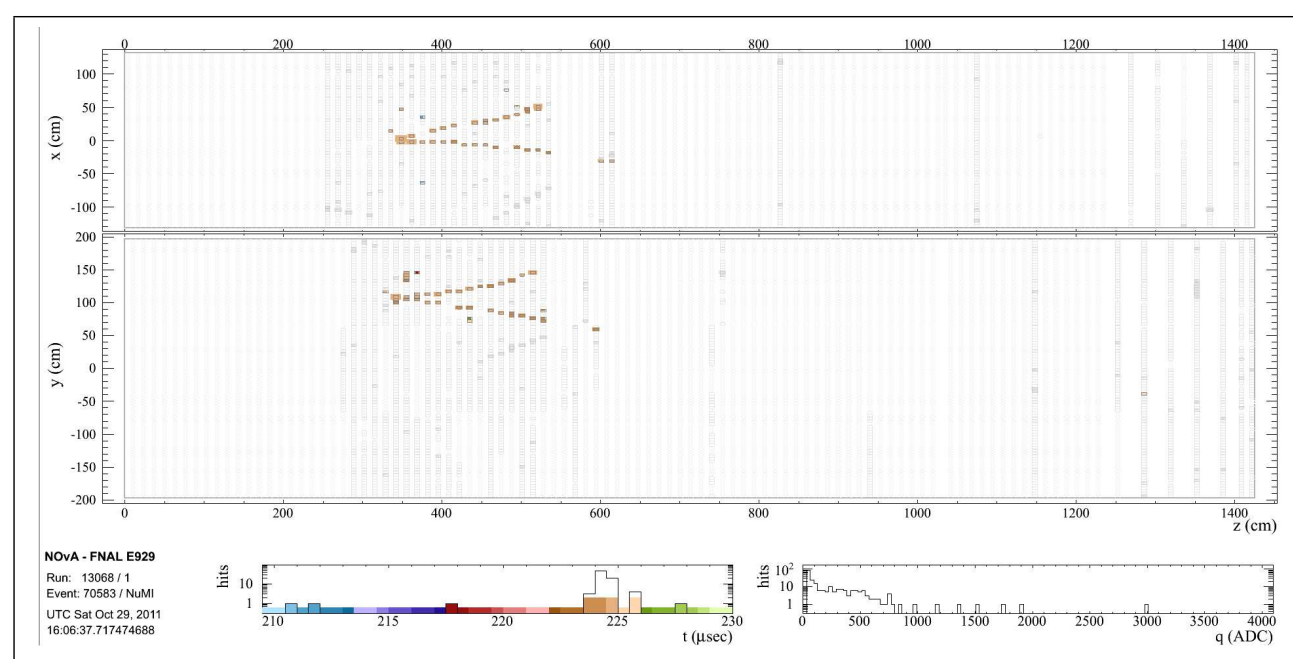

(a)

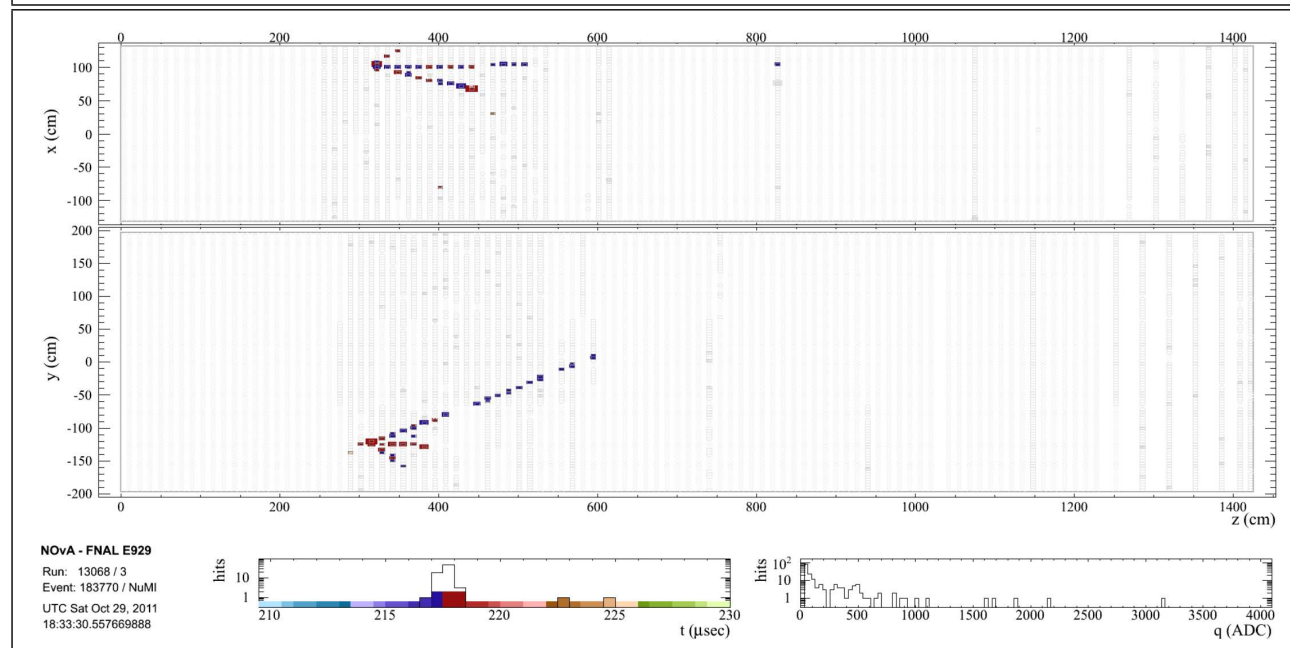

(b)

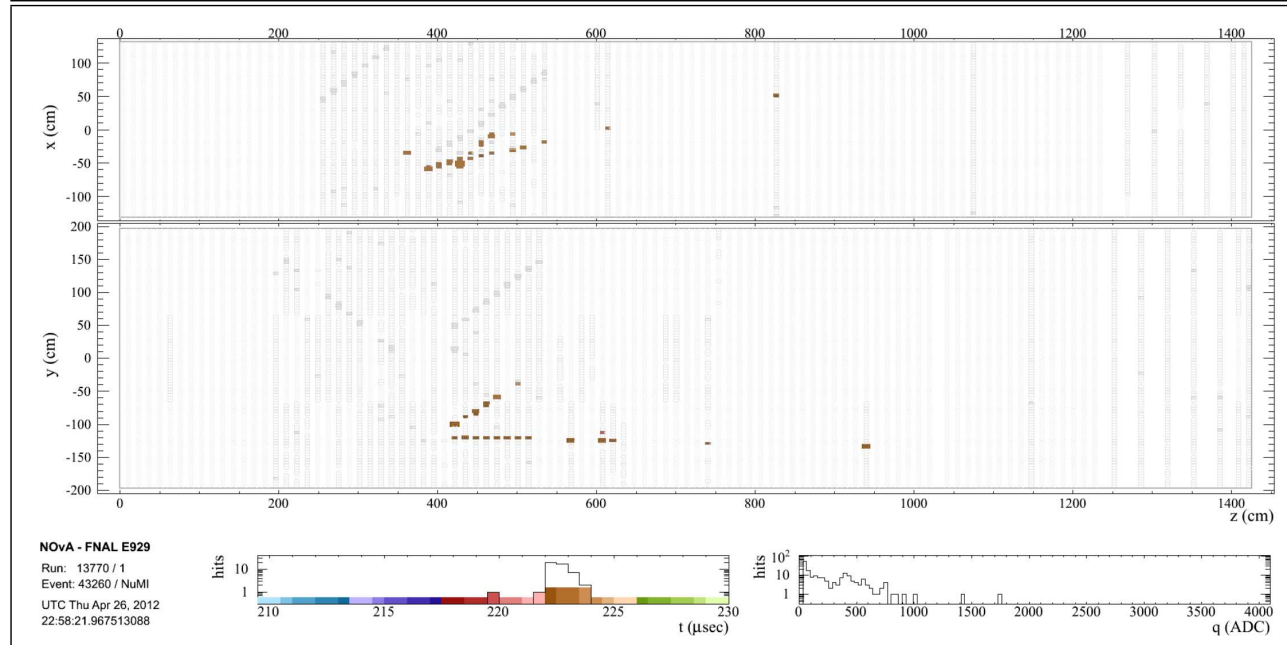

(c)

Figure 8.11 Sample Of Non-QE Neutrino Candidates. (a) Selected neutrino candidate with $1.9 \mathrm{GeV}$ of estimated energy. (b) Selected neutrino candidate with $1.8 \mathrm{GeV}$ of estimated energy. (c) Selected neutrino candidate with $2.0 \mathrm{GeV}$ of estimated energy. 
shown in figure 8.10. The color of the cell hits represent times, and these follow the color code of the timing histogram in the bottom left of figure 8.10. Figure 8.10a presents a selected QE neutrino candidate with $1.0 \mathrm{GeV}$ of reconstructed energy, similar to that presented in figure 8.8a. Figure 8.10b presents a selected QE neutrino candidate with $2.5 \mathrm{GeV}$ of reconstructed energy, similar to that presented in figure 8.8b. Figure 8.10c presents a selected QE neutrino candidate with $3.3 \mathrm{GeV}$ of reconstructed energy, similar to that presented in figure 8.8c. Another sample of selected non-QE neutrino candidates, similar to the one presented in figure 8.9, is shown in figure 8.11. Figure 8.11a presents a selected non-QE neutrino candidate with $1.9 \mathrm{GeV}$ of reconstructed energy, similar to that presented in figure 8.9a. Figure 8.11b presents a selected non-QE neutrino candidate with $1.8 \mathrm{GeV}$ of reconstructed energy, similar to that presented in figure $8.9 \mathrm{~b}$. Figure $8.11 \mathrm{c}$ presents a selected non-QE neutrino candidate with $2.0 \mathrm{GeV}$ of reconstructed energy, similar to that presented in figure $8.9 \mathrm{c}$. These six selected neutrino candidates share similar topologies to the MC simulated ones, hence, MC simulated and data events are likely to share the same type of neutrino interaction, as well as the same final state particles. The full list of selected neutrino candidates is presented in appendix D.

After the subtraction of the cosmic ray background, and the separation of the QE and non-QE samples, the number of neutrino events predicted by the MC simulation is higher, in each case, by about $30 \%$ than is observed in the data, as shown in figure 8.12 . This is agreement with the observation that the MC simulation predicted more muon tracks than appeared in the data. When the MC simulated distribution is normalized to the number of data events, the shapes are quite similar, as shown in figure 8.13.

The distributions presented in figure 8.12 are reconstructed MC simulation and data. There are differences between reconstructed and true MC simulated distributions, as pre- 

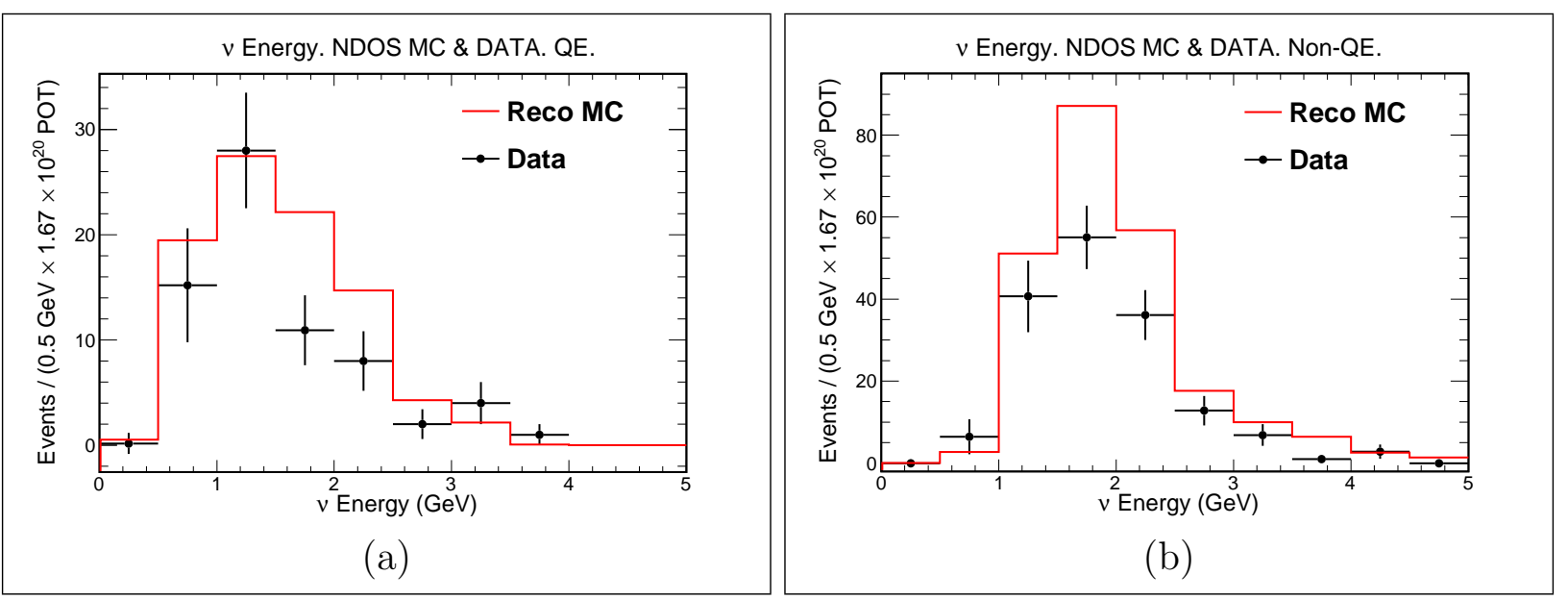

Figure 8.12 Number Of Neutrino Candidates vs. Neutrino Energy, No Cosmic Background. Number of selected neutrino candidate events vs. neutrino energy for (a) QE and (b) non-QE interactions. Reconstructed MC simulation (red) and in-time data (black) energy distributions with a cosmic background subtraction and normalized by POT.
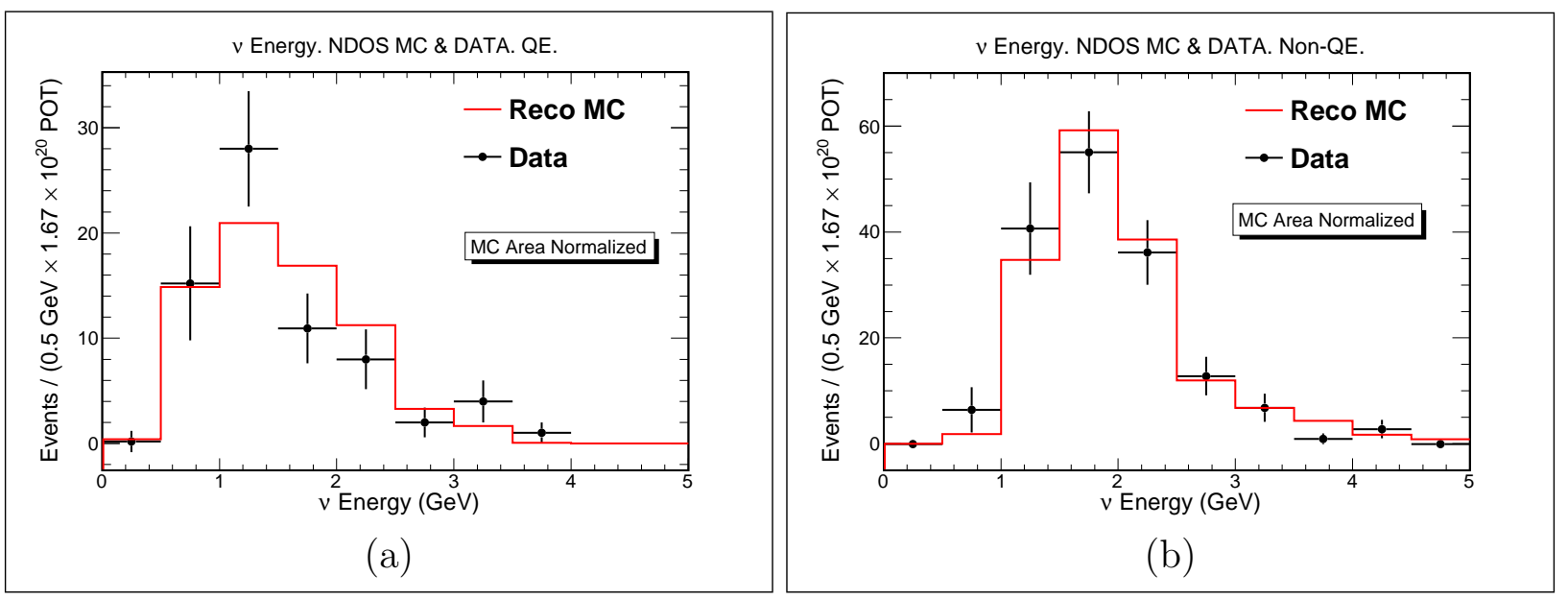

Figure 8.13 Number Of Neutrino Candidates vs. Neutrino Energy, No Cosmic Background, Shape Comparison. (a) QE and (b) non-QE number of neutrino events vs. neutrino energy. Reconstructed MC simulation (red) and in-time data (black) energy distributions. MC simulation areas normalized to data. 
sented in section 6.4, that can only be resolved by the unfolding procedure that accounts for the inefficiencies and resolutions of the detector, and provides the transformation of the reconstructed event energy into corrected energy bins.
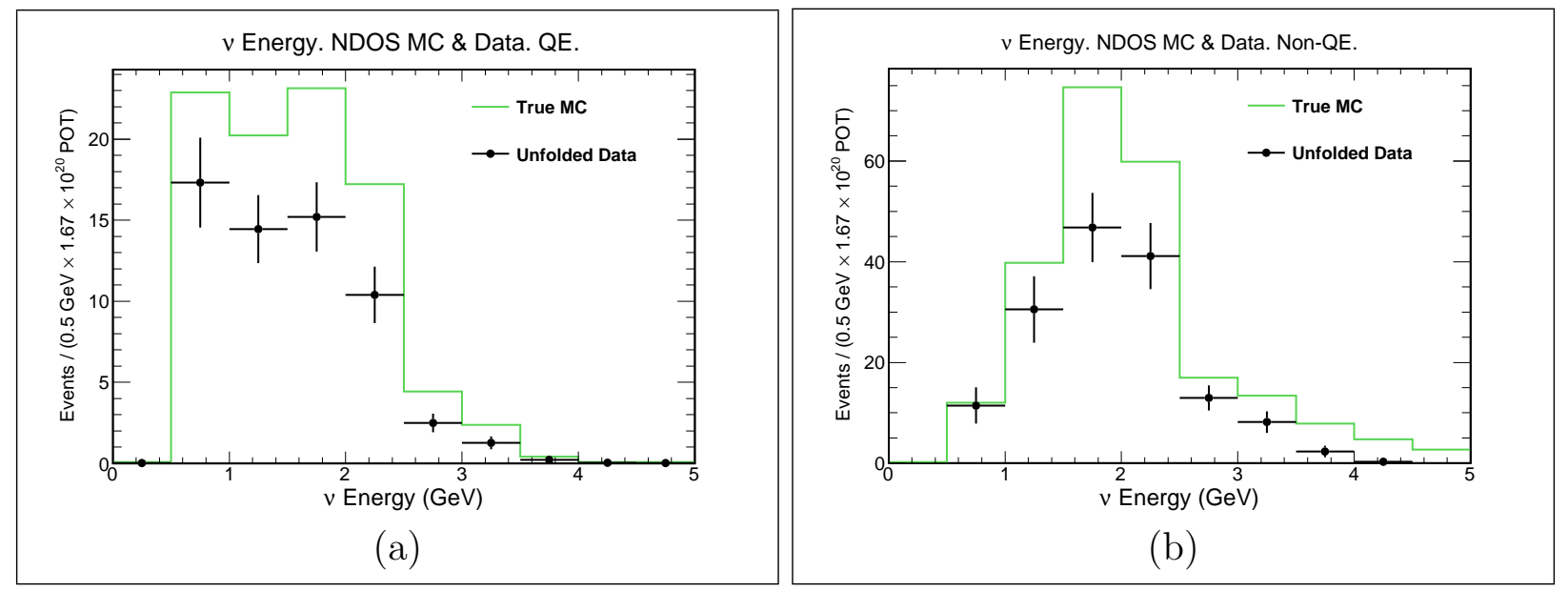

Figure 8.14 QE And Non-QE Unfolded Number Of Neutrino Candidates vs. Neutrino Energy, No Cosmic Background. (a) QE and (b) non-QE number of selected neutrino candidate events vs. neutrino energy after unfolding. True MC simulation (green) and unfolded data (black) energy distributions normalized by POT.

The unfolding algorithm is applied to the data distributions ${ }^{1}$ in figures $8.12 \mathrm{a}$ and $8.12 \mathrm{~b}$, and the results are shown in figure 8.14. The unfolded distributions should be compared to the true $\mathrm{MC}$ simulated distributions. There are $32.4 \%$ more QE predicted events by the MC simulation than unfolded candidate events. There are 35.9\% more non-QE predicted events by the MC simulation than unfolded candidate events. These differences in the predicted and candidate events come, thus, from an extra factor of $1 / 3$ in the normalizations included in the MC simulation. The shapes of the data and MC simulated distributions are in agreement, as see in figure 8.15 , where the MC simulation areas are normalized to data.

\footnotetext{
${ }^{1}$ Figures 8.15 should be compared with figures 8.13 to appreciated the effect of the unfolding procedure.
} 

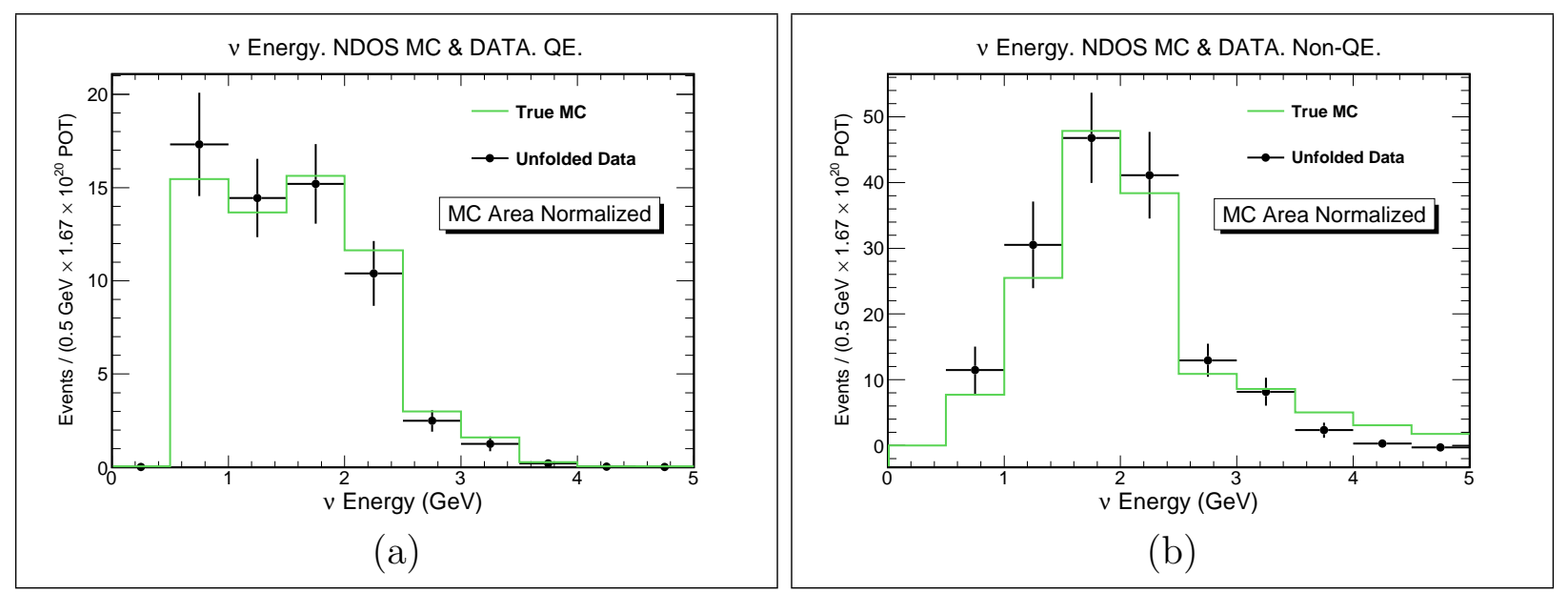

Figure 8.15 QE And Non-QE Unfolded Number Of Neutrino Candidates vs. Neutrino Energy, No Cosmic Background, Shape Comparison. (a) QE and (b) non-QE number of neutrino events vs. neutrino energy after unfolding. True MC simulation (green) and unfolded data (black) energy distributions. MC simulation areas normalized to data.

\subsection{Results}

The inclusive CC event sample is obtained by combining the QE and non-QE neutrino energy distributions presented in figure 8.14. The total number of selected $\nu_{\mu}+\bar{\nu}_{\mu} \mathrm{CC}$ candidate events is: 229.7 per $1.67 \times 10^{20}$ POT. This number is not an integer because it comes from the subtraction of out-of-time data from in-time data. The number of events in the in-time data set is an integer. The out-of-time data set is scaled to the size of the in-time data time window $(11 \mu \mathrm{s})$, and therefore is not necessarily an integer. The sums of the two interactions, which are the reconstructed MC simulation and the data (without unfolding), are shown in figure 8.16a. With the MC simulation normalized to the number of data events, the shapes of the inclusive sample are in good agreement, as seen in figure 8.16b. The true MC simulation, with 328.1 predicted events per $1.67 \times 10^{20}$ POT, exhibits $30 \%$ more predicted events than the unfolded candidate events, as shown in figure 8.17a. Comparing the shapes between the true MC simulated and unfolded data distributions, between $0.5 \mathrm{GeV}$ and $4 \mathrm{GeV}$ in figure $8.17 \mathrm{~b}$, indicates that there is a good agreement among them. The agreement is corroborated 
by the $\chi^{2}$ test, which in this case concludes that $\chi^{2}=5.549$ for $\mathrm{ndf}=6$.
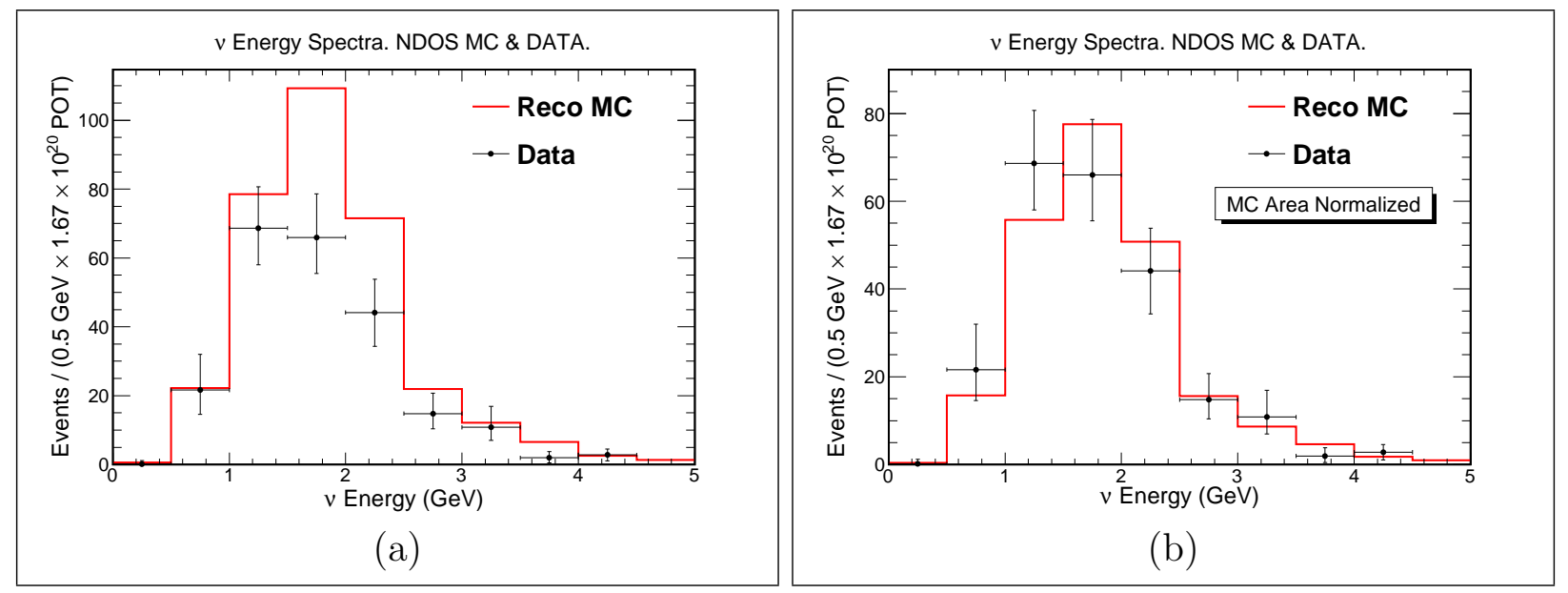

Figure 8.16 Number Of Neutrino Candidates vs. Neutrino Energy, Comparison Of MC Simulation And Data. Energy dependence of the reconstructed MC simulation (red) and data without unfolding (black) normalized by (a) POT, and (b) with MC simulation areas normalized to data.
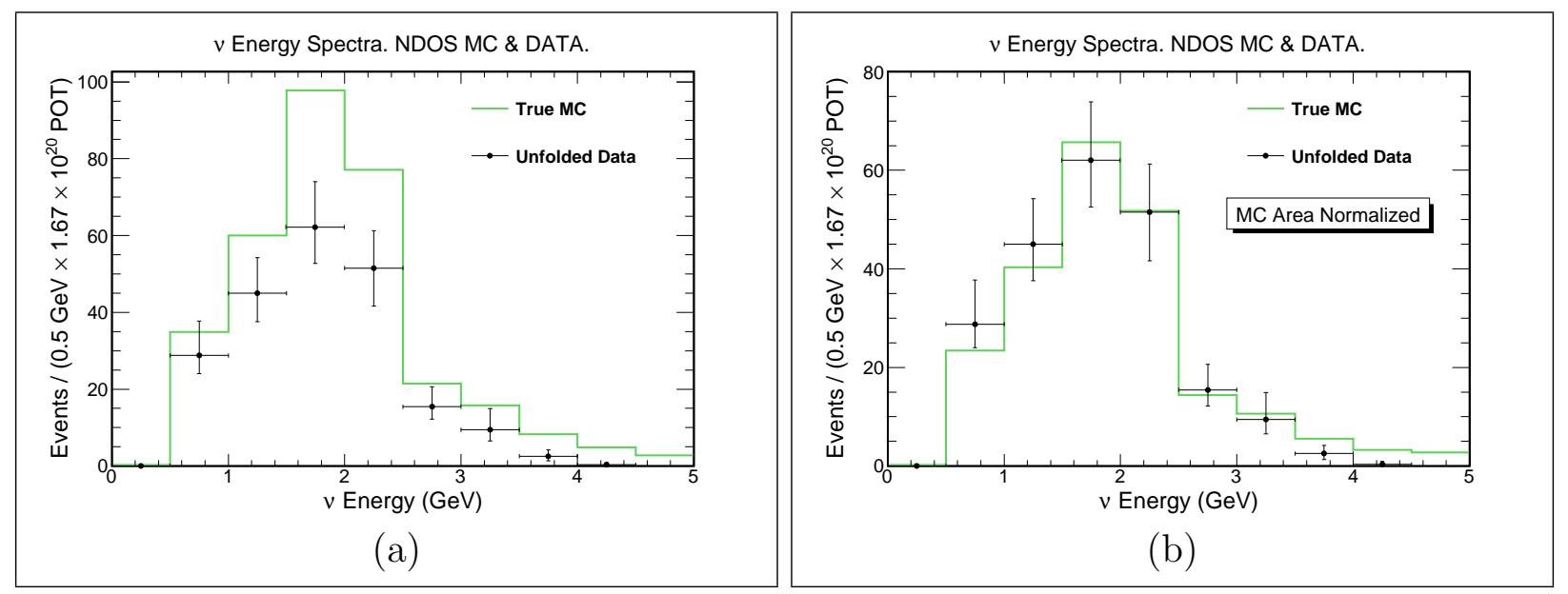

Figure 8.17 Unfolded Number Of Neutrino Candidates vs. Neutrino Energy, Comparison Of MC Simulation And Data. True MC simulation (green) and unfolded data (black) normalized by (a) POT, and (b) with MC simulation areas normalized to data.

The number of selected CC candidate events per energy bin $\left(N_{\mathrm{CC}}^{\mathrm{bin}}\right)$ in figure 8.16 has no cosmic background ${ }^{2}, 2.9 \%$ of overall NC background, and $1.4 \%$ of overall $\nu_{e}+\bar{\nu}_{e} \mathrm{CC}$ background (see section 6.1). Table 8.5, at the end of the chapter, summarizes the percent-

\footnotetext{
${ }^{2}$ Due to cosmic background subtraction.
} 
ages of NC background per energy bin, and table 8.6, at the end of the chapter, summarizes the percentages of $\nu_{e}+\bar{\nu}_{e} \mathrm{CC}$ background per energy bin. These two tables feature the backgrounds present in the $N_{\mathrm{CC}}^{\mathrm{bin}}$.

\begin{tabular}{|c|c|c|c|c|c|c|c|}
\hline$\nu$ kind $\backslash \mathrm{E}(\mathrm{GeV})$ & $0.5-1.0$ & $1.0-1.5$ & $1.5-2.0$ & $2.0-2.5$ & $2.5-3.0$ & $3.0-3.5$ & $3.5-4.0$ \\
\hline \hline$\nu_{\mu} Q E: M_{q}^{\nu \mu}$ & 16.8 & 13.2 & 14.9 & 11.2 & 2.3 & 1.3 & 0.2 \\
\hline$\nu_{\mu}$ Non-QE: $M_{n}^{\nu \mu}$ & 1.8 & 19.8 & 39.0 & 34.1 & 8.4 & 5.2 & 1.6 \\
\hline $\bar{\nu}_{\mu} Q E: M_{q}^{\nu \mu}$ & 8.9 & 6.5 & 3.4 & 2.5 & 1.6 & 1.0 & 0.1 \\
\hline $\bar{\nu}_{\mu}$ Non-QE: $M_{n}^{\nu \mu}$ & 1.2 & 5.5 & 4.7 & 3.7 & 3.2 & 1.9 & 0.6 \\
\hline \hline$N_{\mathrm{CC}}^{\text {bin }}$ & 28.8 & 45.0 & 62.0 & 51.5 & 15.4 & 9.4 & 2.5 \\
\hline
\end{tabular}

Table 8.3 Candidate Events With Background. Number of selected candidate events per neutrino type and interaction type. The cosmic background was subtracted, though these numbers still reflect the $\mathrm{NC}$ and $\nu_{e}+\bar{\nu}_{e} \mathrm{CC}$ background presence. The last row, $N_{\mathrm{CC}}^{\mathrm{bin}}$, is the total number of selected candidate events per energy bin. See equation (8.3) for the definition of the $M_{q}^{\nu}$ and $M_{n}^{\nu}$.

The total number of selected candidate events, $N_{T}$, is the sum of the CC QE $\left(N_{q}\right)$ and CC non-QE $\left(N_{n}\right)$ neutrino candidates (see equations (8.5) and (8.6)), and each of these two numbers is the sum of the $\nu_{\mu}$ and $\bar{\nu}_{\mu}$ contributions:

$$
N_{T}=N_{q}^{\nu \mu}+N_{n}^{\nu \mu}+N_{q}^{\bar{\nu} \mu}+N_{n}^{\bar{\nu} \mu} .
$$

After the background subtraction is applied, the $M_{q}^{\nu}$ and $M_{n}^{\nu}$ in table 8.3 are substituted into equations $(8.5,8.6)$ yielding $N_{q}$ and $N_{n}$ respectively.

The final results, the number of selected $\nu_{\mu}+\bar{\nu}_{\mu}$ CC candidate events per energy bin $\left(N_{T}^{\text {bin }}\right)$, are summarized in table 8.4. Statistical and systematic uncertainties are of the same order, with the systematic error typically larger. The total uncertainty is the sum in quadrature of the two types of uncertainties, as presented in the full discussion on systematic uncertainties in chapter 7 . These $N_{\mathrm{CC}}^{\mathrm{bin}}$ are about $2 / 3$ of the prediction done by the MC 


\begin{tabular}{|c|c|c|c|c|}
\hline$\nu$ Energy $(\mathrm{GeV})$ & Candidates $/ 1.67 \times 10^{20}$ POT & stat. error & sys. error & total error \\
\hline \hline 0.5 to 1.0 & 28.28 & 4.47 & +7.60 & +8.82 \\
\hline 1.0 to 1.5 & 42.30 & 6.50 & -1.38 & -4.68 \\
\hline 1.5 to 2.0 & 58.54 & 6.79 & +8.43 & -6.94 \\
\hline 2.0 to 2.5 & 48.56 & 6.42 & -5.82 & +8.62 \\
\hline 2.5 to 3.0 & 14.06 & 2.29 & -6.75 & -9.94 \\
\hline 3.0 to 3.5 & 8.63 & 1.98 & -1.81 & -9.32 \\
\hline 3.5 to 4.0 & 2.31 & 1.06 & -1.75 & -2.96 \\
\hline
\end{tabular}

Table 8.4 Number Of Candidate Events. Selected $\nu_{\mu}+\bar{\nu}_{\mu}$ CC candidates per neutrino energy bin. Statistical and systematic errors are shown in independent columns. The total error combining the statistical and systematic errors are shown in the last column.

simulation. Chapter 9 addresses the possible causes of the disagreement between NDOS data and the MC simulation.

\begin{tabular}{|c|c|c|c|c|c|c|c|}
\hline$\nu$ kind $\backslash \mathrm{E}(\mathrm{GeV})$ & $0.5-1.0$ & $1.0-1.5$ & $1.5-2.0$ & $2.0-2.5$ & $2.5-3.0$ & $3.0-3.5$ & $3.5-4.0$ \\
\hline \hline$\nu_{\mu} \mathrm{QE}$ & $0.1 \%$ & 0 & $0.9 \%$ & $0.5 \%$ & $1.1 \%$ & $1.1 \%$ & $0 \%$ \\
Uncertainty & $\pm 0.1 \%$ & 0 & $\pm 0.9 \%$ & $\pm 0.5 \%$ & $\pm 1.0 \%$ & $\pm 1.0 \%$ & $0 \%$ \\
\hline$\nu_{\mu}$ Non-QE & 0 & $0.6 \%$ & $1.7 \%$ & $3.9 \%$ & $4.0 \%$ & $6.4 \%$ & $7.1 \%$ \\
Uncertainty & 0 & $\pm 0.6 \%$ & $\pm 1.3 \%$ & $\pm 2.0 \%$ & $\pm 2.1 \%$ & $\pm 2.5 \%$ & $\pm 2.7 \%$ \\
\hline $\bar{\nu}_{\mu}$ Non-QE & 0 & $0.1 \%$ & $0.1 \%$ & $0.2 \%$ & $1.0 \%$ & $0.5 \%$ & $1.1 \%$ \\
Uncertainty & 0 & $\pm 0.1 \%$ & $\pm 0.1 \%$ & $\pm 0.2 \%$ & $\pm 1.0 \%$ & $\pm 0.5 \%$ & $\pm 1.0 \%$ \\
\hline$\nu_{e}$ Non-QE & 0 & 0 & $0.1 \%$ & $0.1 \%$ & $0.3 \%$ & $0.2 \%$ & $0.4 \%$ \\
Uncertainty & 0 & 0 & $\pm 0.1 \%$ & $\pm 0.1 \%$ & $\pm 0.3 \%$ & $\pm 0.2 \%$ & $\pm 0.4 \%$ \\
\hline
\end{tabular}

Table 8.5 Neutral Current Background. The NC background per energy bin as a function of the interaction type. The rows in this table represent the interaction type. 


\begin{tabular}{|c|c|c|c|c|c|c|c|}
\hline$\nu$ kind $\backslash \mathrm{E}(\mathrm{GeV})$ & $0.5-1.0$ & $1.0-1.5$ & $1.5-2.0$ & $2.0-2.5$ & $2.5-3.0$ & $3.0-3.5$ & $3.5-4.0$ \\
\hline \hline$\nu_{e}+\bar{\nu}_{e} Q E$ & $1.6 \%$ & $3.0 \%$ & $2.4 \%$ & $0.9 \%$ & $0.5 \%$ & $1.1 \%$ & 0 \\
Uncertainty & $\pm 1.3 \%$ & $\pm 1.7 \%$ & $\pm 1.5 \%$ & $0 \pm .9 \%$ & $\pm 0.5 \%$ & $\pm 1.0 \%$ & 0 \\
\hline$\nu_{e}+\bar{\nu}_{e}$ Non-QE & $0.8 \%$ & $1.2 \%$ & $0.6 \%$ & $0.5 \%$ & $1.7 \%$ & $1.4 \%$ & $0.2 \%$ \\
Uncertainty & $\pm 0.8 \%$ & $\pm 1.1 \%$ & $\pm 0.6 \%$ & $\pm 0.5 \%$ & $\pm 1.3 \%$ & $\pm 1.2 \%$ & $\pm 0.2 \%$ \\
\hline
\end{tabular}

Table 8.6 Electron Neutrino Background. The $\nu_{e}+\bar{\nu}_{e}$ CC background per energy bin as a function of the interaction type. The rows in this table represent the interaction type. 


\section{Chapter 9}

\section{Discussion And Final Remarks}

The number of selected events $\left(N_{\nu_{\mu}}\right)$ found in section 8.2 is a function of the neutrino flux, and the cross section between the incident neutrinos and the number of target nuclei $(T)$ [113]:

$$
N_{\nu \mu}\left(E_{\nu}\right)=T \sigma\left(E_{\nu}\right) \Phi\left(E_{\nu}\right)
$$

To investigate which of the two considered quantities $\left(\sigma\left(E_{\nu}\right)\right.$ or $\left.\Phi\left(E_{\nu}\right)\right)$ is responsible for the excess of neutrino events predicted by the MC simulation, as discussed in section 8.2, the following sections present the calculations of both quantities using the results already obtained.

\subsection{Flux Calculation}

The flux calculation presented in this section assumes that the cross sections used by GENIE to simulated neutrino interactions are in agreement with the current measurements ${ }^{1}$. The flux of charged kaons produced at the NuMI target can be constrained using neutrinos detected with NDOS since about $93 \%$ of the neutrinos in the $2 \mathrm{GeV}$ peak come from charged kaon decays. Figure 9.1 shows the longitudinal momentum (figure 9.1a) and the transverse momentum (figure 9.1b) of the charged kaons produced at the NuMI target as a function of

\footnotetext{
${ }^{1}$ See section 9.1.2 for a discussion on the cross sections used in the MC simulation.
} 


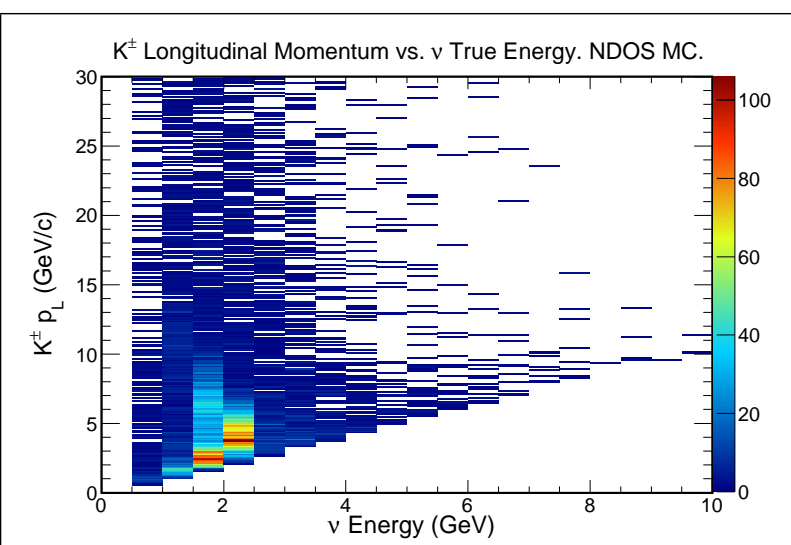

(a)

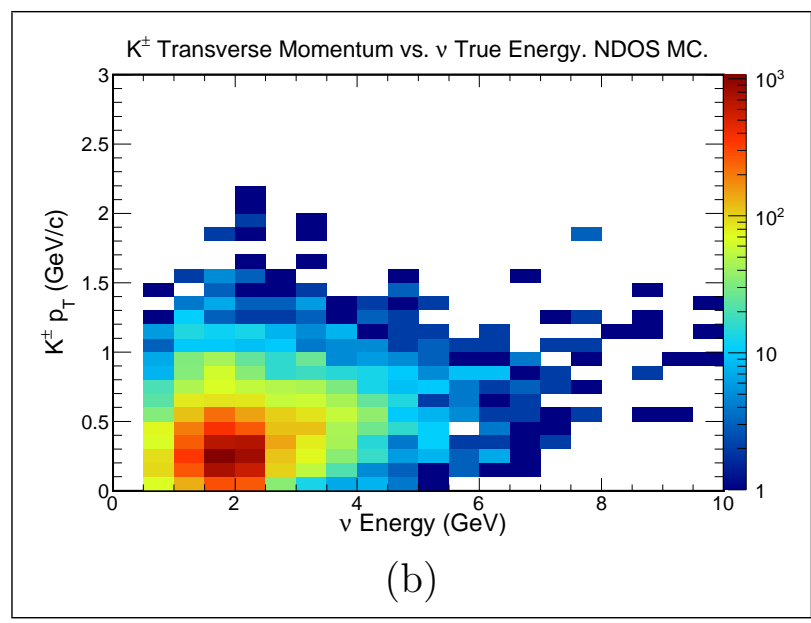

(b)

Figure 9.1 Charged Kaon Longitudinal And Transverse Momenta. (a) Longitudinal and (b) transverse momenta of charged kaons produced at the NuMI target as a function of the daughter neutrino energies. Event selection criteria applied to these MC simulated sample. MC simulation.

the daughter neutrino energy ${ }^{2}$. The average longitudinal momentum $\left(p_{L}\right)$ of charged kaons when the daughter neutrino energy is restricted to:

$$
1.5 \mathrm{GeV}<E_{\nu}<3.5 \mathrm{GeV}
$$

is $6.61 \mathrm{GeV} / \mathrm{c}$. The average transverse momentum $\left(p_{T}\right)$ of charged kaons for the neutrino energies shown in equation (9.2) is $0.34 \mathrm{GeV}$. The average momentum of these charged kaons is therefore $6.62 \mathrm{GeV} / \mathrm{c}$. The neutrinos detected with NDOS at $110 \mathrm{mrad}$ off NuMI axis, thus, constrain the flux of charged kaons produced at the NuMI target with average momentum of $(6.62 \pm 1.72) \mathrm{GeV} / \mathrm{c}$.

The sum of all the neutrinos coming from: charged kaon, charged pion, muon, and $K_{L}$ decays was presented in figure 3.10. In addition, the neutrinos coming from charged kaon decays are dominant for energies higher than $1.5 \mathrm{GeV}$, as shown in figure 9.2. This figure is an updated version of figure 3.12 after all the event selection is applied to the MC simulated

\footnotetext{
${ }^{2}$ Here $K$ means $K^{+}$and $K^{-}$added together.
} 


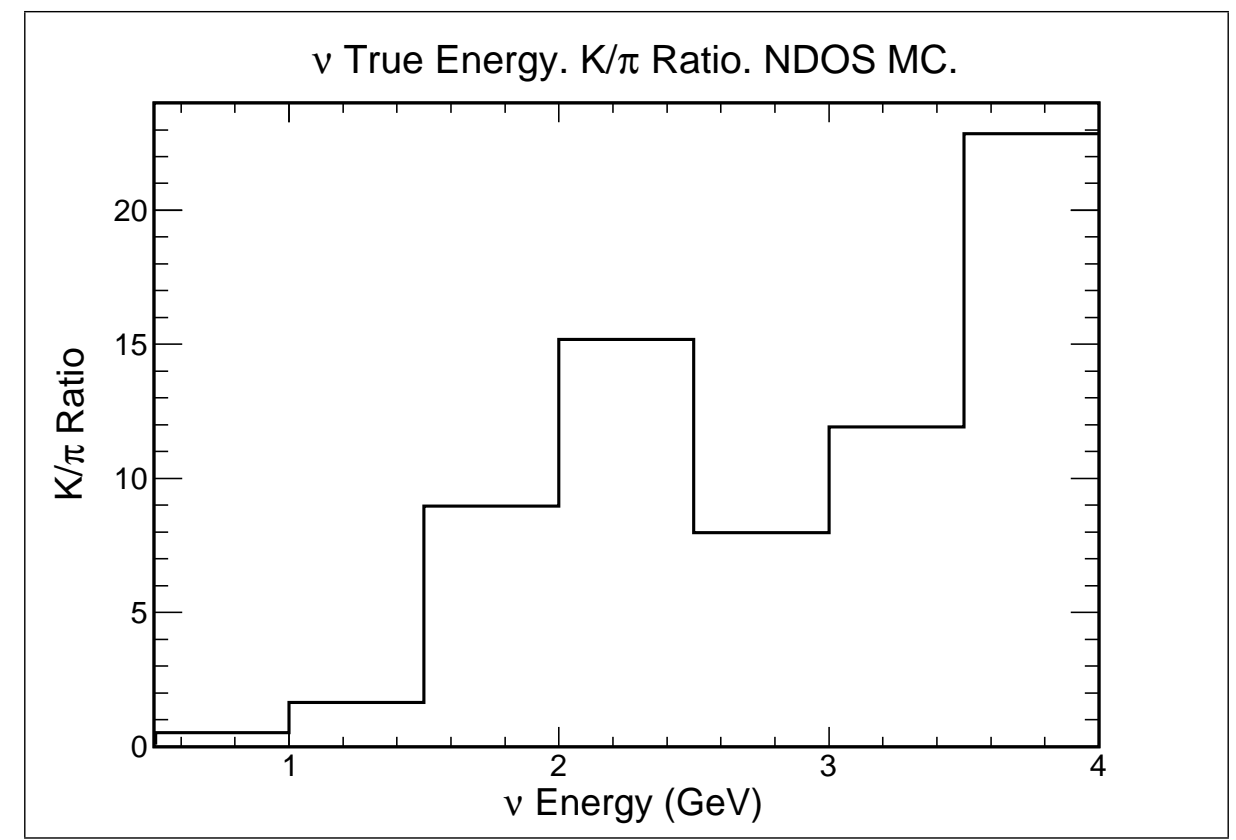

Figure 9.2 Ratio Of Neutrinos From Charged Kaon Decays Over Neutrinos From Charged Pion Decays After Event Selection Criteria Applied. MC simulation.

sample. For these energies, the overall (integrated) background of neutrinos coming from charged pion decays is $7.7 \%$, and from muons $+K_{L}$ decays is $1.1 \%$. Table 9.1 summarizes the percentages of charged pions and muons $+K_{L}$ background per energy bin. The statistically significant data in figure 8.16 have energies less than $3.5 \mathrm{GeV}$, so the charged kaons flux measurement is calculated using data between $1.5 \mathrm{GeV}$ and $3.5 \mathrm{GeV}$.

\begin{tabular}{|c|c|c|c|c|c|c|c|}
\hline Decay $\backslash \mathrm{E}(\mathrm{GeV})$ & $0.5-1.0$ & $1.0-1.5$ & $1.5-2.0$ & $2.0-2.5$ & $2.5-3.0$ & $3.0-3.5$ & $3.5-4.0$ \\
\hline \hline$\pi^{+} \rightarrow \nu_{\mu}, \mathrm{QE}$ & $62.9 \%$ & $20.6 \%$ & $5.7 \%$ & $3.1 \%$ & $2.1 \%$ & $6.3 \%$ & $33.3 \%$ \\
\hline$\pi^{+} \rightarrow \nu_{\mu}$, Non-QE & $37.5 \%$ & $13.6 \%$ & $4.1 \%$ & $2.6 \%$ & $3.4 \%$ & $3.0 \%$ & $1.1 \%$ \\
\hline$\pi^{-} \rightarrow \bar{\nu}_{\mu}, \mathrm{QE}$ & $40.5 \%$ & $17.9 \%$ & $8.9 \%$ & $7.8 \%$ & $19.1 \%$ & $7.4 \%$ & $33.3 \%$ \\
\hline$\pi^{-} \rightarrow \bar{\nu}_{\mu}$, Non-QE & $39.6 \%$ & $8.0 \%$ & $3.0 \%$ & $2.6 \%$ & $4.0 \%$ & $5.5 \%$ & $3.2 \%$ \\
\hline$\mu^{-}$or $K_{L} \rightarrow \nu_{\mu}, \mathrm{QE}$ & $2.2 \%$ & $1.1 \%$ & $0.6 \%$ & $0.5 \%$ & $1.1 \%$ & $1.1 \%$ & 0 \\
\hline$\mu^{-}$or $K_{L} \rightarrow \nu_{\mu}$, Non-QE & $1.7 \%$ & $0.8 \%$ & $0.4 \%$ & $0.3 \%$ & $0.6 \%$ & $0 \%$ & 0 \\
\hline$\mu^{+}$or $K_{L} \rightarrow \bar{\nu}_{\mu}, \mathrm{QE}$ & $1.9 \%$ & $1.2 \%$ & $1.1 \%$ & $0.5 \%$ & $0.5 \%$ & $1.0 \%$ & 0 \\
\hline$\mu^{+}$or $K_{L} \rightarrow \bar{\nu}_{\mu}$, Non-QE & $0.8 \%$ & $0.2 \%$ & $0.2 \%$ & $0.5 \%$ & $0.3 \%$ & $0.5 \%$ & 0 \\
\hline
\end{tabular}

Table 9.1 Background To Neutrinos From Kaon Decays. Percentage of neutrinos not coming from charged kaon decays per energy bin. The rows in this table represent the decaying particle that produces the neutrino. 


\begin{tabular}{|c|c|c|c|c|c|c|c|}
\hline int. $\backslash \mathrm{E}(\mathrm{GeV})$ & $0.5-1.0$ & $1.0-1.5$ & $1.5-2.0$ & $2.0-2.5$ & $2.5-3.0$ & $3.0-3.5$ & $3.5-4.0$ \\
\hline \hline$\nu_{\mu} Q E$ & 9.8 & 9.9 & 13.8 & 10.8 & 2.3 & 1.3 & 0.2 \\
\hline$\nu_{\mu}$ Non-QE & 1.3 & 14.9 & 35.6 & 31.5 & 7.4 & 4.7 & 1.5 \\
\hline $\bar{\nu}_{\mu} Q E$ & 6.5 & 5.2 & 3.1 & 2.4 & 1.3 & 1.0 & 0.1 \\
\hline $\bar{\nu}_{\mu}$ Non-QE & 0.9 & 4.8 & 4.5 & 3.6 & 3.0 & 1.8 & 0.6 \\
\hline \hline$N_{K}^{\text {bin }}$ & $18.5_{-3.1}^{+7.5}$ & $34.7_{-5.6}^{+7.3}$ & $57.1_{-8.6}^{+10.8}$ & $48.3 \pm 9.2$ & $14.0_{-2.9}^{+4.8}$ & $8.7_{-2.7}^{+5.1}$ & $2.4_{-1.2}^{+1.6}$ \\
\hline
\end{tabular}

Table 9.2 Candidate Events From Kaon Decays. Number of candidate events per neutrino type and interaction type, when the parent meson is a charged kaon. Each row in this table represents a neutrino type associated with an interaction type. The last row, $N_{K}^{\text {bin }}$, is the total number of selected candidate events per energy bin. These numbers come after NC, $\nu_{e}+\bar{\nu}_{e}$, and charged pions + muons $+K_{L}$ background subtraction. Tables 8.5 and 8.6 summarize these backgrounds.

The charged kaon decays of interest are:

$$
\begin{aligned}
K^{+} & \rightarrow \nu_{\mu}+\mu^{+}, \\
& \rightarrow \nu_{\mu}+\mu^{+}+\pi^{0}, \\
K^{-} & \rightarrow \bar{\nu}_{\mu}+\mu^{-}, \\
& \rightarrow \bar{\nu}_{\mu}+\mu^{-}+\pi^{0} .
\end{aligned}
$$

To find the total number of candidates coming from charged kaon decays $\left(N_{K}\right)$, the charged pions + muons $+K_{L}$ background is subtracted from $N_{T}$ (see equation (8.13)). Table 9.2 summarized the various $N_{K}^{\text {bin }}$. The flux is a function of: the number of $\nu_{\mu}+\bar{\nu}_{\mu}, N_{K}$, detected with NDOS, their CC cross sections $(\sigma)$ with respect to the nuclei in NDOS, the number of target atoms $(T)$ in the VR, and the reconstruction efficiency $(\epsilon)[113]$ :

$$
\Phi=\sum_{i} \frac{N_{i}^{K}}{\epsilon_{i} \sigma_{i} T} .
$$

All these quantities are a function of the energy bin, $i$. The total flux, $\Phi$, is the sum of the 
individual fluxes per energy bin.

\subsubsection{Number Of Atoms In The Target Region}

The number of atoms available to interact with neutrinos in the VR is calculated using $\mathrm{NO} \nu \mathrm{A}$ design information $[114,83]$, interacting with $\mathrm{NO} \nu \mathrm{A}$ PVC extrusions experts [115], and measuring dimensions and weights of spare NDOS PVC extrusions kept at Argonne National Laboratory. This number of atoms is confined to the NDOS target region (TR) with coordinates restricted by:

$$
\begin{gathered}
|X|<106 \mathrm{~cm}, \\
|Y|<172 \mathrm{~cm}, \\
288 \mathrm{~cm}<Z<452 \mathrm{~cm} .
\end{gathered}
$$

The PVC mass inside the TR ( $\left.m_{\mathrm{pvc}}\right)$ is $4.90 \%$ of the total PVC mass, $M_{\mathrm{pvc}}$ :

- $M_{\mathrm{pvc}}=(38.3 \pm 1.9) \times 10^{3} \mathrm{Kg}$.

- $m_{\mathrm{pvc}}=(1.9 \pm 0.1) \times 10^{3} \mathrm{Kg}$.

The scintillator mass inside the TR $\left(m_{l}\right)$ is $4.94 \%$ of the total scintillator mass, $M_{l}$ :

- $M_{l}=(91.3 \pm 4.6) \times 10^{3} \mathrm{Kg}$.

- $m_{l}=(4.5 \pm 0.2) \times 10^{3} \mathrm{Kg}$.

Even though the amount of WLS fiber, glue, and air within the TR is very small, it is calculated as well:

- WLS fiber mass: $m_{\mathrm{wls}}=(5.1 \pm 0.3) \mathrm{Kg}$. 
- Glue mass: $m_{g}=(3.1 \pm 0.2) \mathrm{Kg}$.

- Air mass: $m_{a}=(6.0 \pm 0.3) \times 10^{-3} \mathrm{Kg}$.

The NO $\nu$ A software has a function, GeometryBase::TotalMass, which provides the numbers discussed above:

- $\mathrm{MC}$ simulated PVC mass: $M_{\mathrm{pvc}}^{\mathrm{MC}}=39.9 \times 10^{3} \mathrm{Kg}$.

- $\mathrm{MC}$ simulated scintillator mass: $M_{l}^{\mathrm{MC}}=87.7 \times 10^{3} \mathrm{Kg}$.

\begin{tabular}{|c|c|c|c|c|c|}
\hline Component & Molar Mass $(\mathrm{g})$ & fraction $(f)$ & Mass $(\mathrm{Kg})$ & Moles & Molecules \\
\hline \hline Scintillator & & & & & \\
$\mathrm{CH}_{2}$ & 14 & 0.9464 & 4270.6 & $3.02 \times 10^{5}$ & $1.83 \times 10^{29}$ \\
$\mathrm{C}_{9} \mathrm{H}_{12}$ & 120 & 0.0523 & 236 & $1.97 \times 10^{3}$ & $1.18 \times 10^{27}$ \\
$\mathrm{C}_{15} \mathrm{H}_{11} \mathrm{NO}$ & 221 & 0.0014 & 6.3 & $2.84 \times 10$ & $1.71 \times 10^{25}$ \\
$\mathrm{C}_{24} \mathrm{H}_{22}$ & 310 & 0.0002 & 0.7 & 2.33 & $1.4 \times 10^{24}$ \\
\hline $\mathrm{PVC}$ & & & & & \\
$\mathrm{C}_{2} \mathrm{H}_{3} \mathrm{Cl}$ & 62 & 0.8 & 1502 & $2.42 \times 10^{4}$ & $1.46 \times 10^{28}$ \\
$\mathrm{TiO}_{2}$ & 80 & 0.15 & 281.6 & $3.52 \times 10^{3}$ & $2.12 \times 10^{27}$ \\
$\mathrm{C}_{36} \mathrm{H}_{70} \mathrm{O}_{4} \mathrm{Ca}$ & 607 & 0.006 & 11.3 & $1.86 \times 10$ & $1.12 \times 10^{25}$ \\
$\mathrm{C}_{20} \mathrm{H}_{24}$ & 264 & 0.009 & 16.9 & $6.4 \times 10$ & $3.85 \times 10^{25}$ \\
$\mathrm{C}_{12} \mathrm{H}_{20} \mathrm{O}_{5}$ & 244 & 0.002 & 3.8 & $1.54 \times 10$ & $9.27 \times 10^{24}$ \\
$\mathrm{C}_{21} \mathrm{H}_{42} \mathrm{O}_{4}$ & 359 & 0.002 & 3.8 & $1.05 \times 10$ & $6.3 \times 10^{24}$ \\
$\mathrm{C}_{5} \mathrm{H}_{8} \mathrm{O}_{2}$ & 100 & 0.031 & 58.2 & $5.82 \times 10^{2}$ & $3.5 \times 10^{26}$ \\
\hline $\mathrm{Air}$ & & & & & \\
$\mathrm{N}$ & 14 & 0.78 & $5.0 \times 10^{-3}$ & 0.33 & $2.01 \times 10^{23}$ \\
$\mathrm{O}$ & 16 & 0.21 & $1.0 \times 10^{-3}$ & 0.08 & $4.72 \times 10^{22}$ \\
Ar & 40 & 0.01 & $1.0 \times 10^{-4}$ & $1.35 \times 10^{-3}$ & $8.13 \times 10^{20}$ \\
\hline WLS Fiber & & & & & \\
$\mathrm{C}_{6} \mathrm{H}_{5} \mathrm{CH}$ & 90 & 1 & 5.1 & $5.68 \times 10$ & $3.42 \times 10^{25}$ \\
\hline Glue & & & & & \\
$\mathrm{C}$ & 12 & 1 & 3.1 & $2.59 \times 10^{2}$ & $1.56 \times 10^{26}$ \\
\hline
\end{tabular}

Table 9.3 Chemical Composition Of The Prototype Detector. NDOS chemical composition, and number of atoms or molecules in each of the elements or compounds that are inside the target region defined in equation (9.5).

The chemical composition of the NDOS components [116] is summarized in table 9.3. With the identity of all the molecules present in the NDOS compounds, the procedure to 
obtain the target mass is straight forward. The molar mass $\left(m_{m}\right)$ of molecules or atoms is the mass of one mole of such substance. Table 9.3 contains the proportions $(f)$ of each element or molecule in each of the compounds of NDOS. The number of target atoms or molecules $\left(T_{x}\right)$, inside the TR, of a given element or compound with mass $m_{x}$ is:

$$
T_{x}=\frac{m_{x} f n_{a}}{m_{m}}
$$

where $n_{a}$ is Avogadro's number. The known cross sections are for individual atoms, hence the number of atoms in each molecule is known from its chemical composition. Table 9.3 summarizes the number of atoms or molecules that make up the mass of the NDOS TR.

\subsubsection{Monte Carlo Cross Sections}

GENIE has tables with the cross sections of many processes labeled under the categories: $\mathrm{QE}$ and non-QE, where GENIE labels the individual non-QE processes as: resonance, deep inelastic or coherent. Such tables contain the various cross sections as a function of the neutrino energy as well as the target nucleus. Since the neutrino energies recorded in the tables are discrete, GENIE interpolates the recorded cross sections in order to obtain the cross section of a particular event for the given energy. The GENIE cross sections used in this analysis are displayed in figure 9.3. All the non-QE cross sections per nucleus are added together.

The cross sections shown in figure 9.3 are calculated assuming that experimental results

on cross sections per nucleon can be extrapolated to give nuclei cross sections. The uncertainties given by GENIE to these cross sections are around 10\%, from the green band shown in figure 9.4. This figure also summarizes the measurements used to calculate the GENIE 

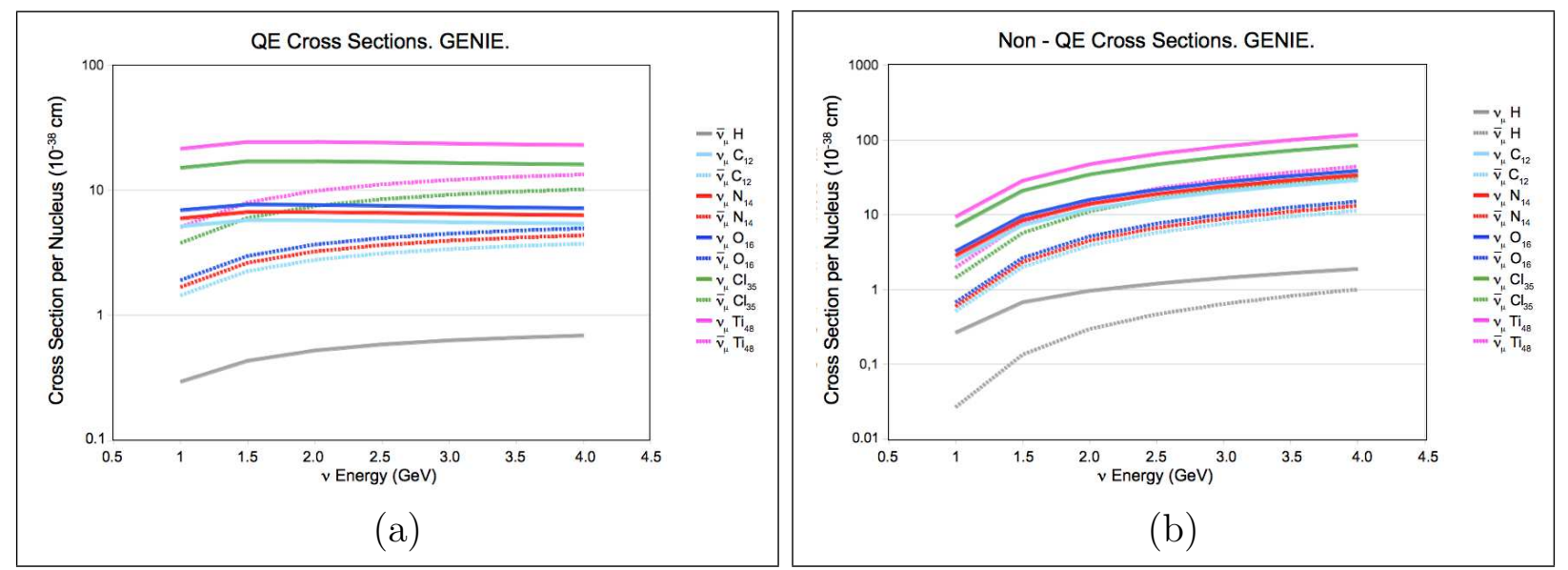

Figure 9.3 GENIE Cross Sections As A Function Of Neutrino Energy. Cross section for each of the elements present in the NDOS target region's mass. The color code is in the right side of the figures, solid lines are for neutrinos and dashed lines are for antineutrinos. Summary of (a) QE and (b) non-QE cross sections.

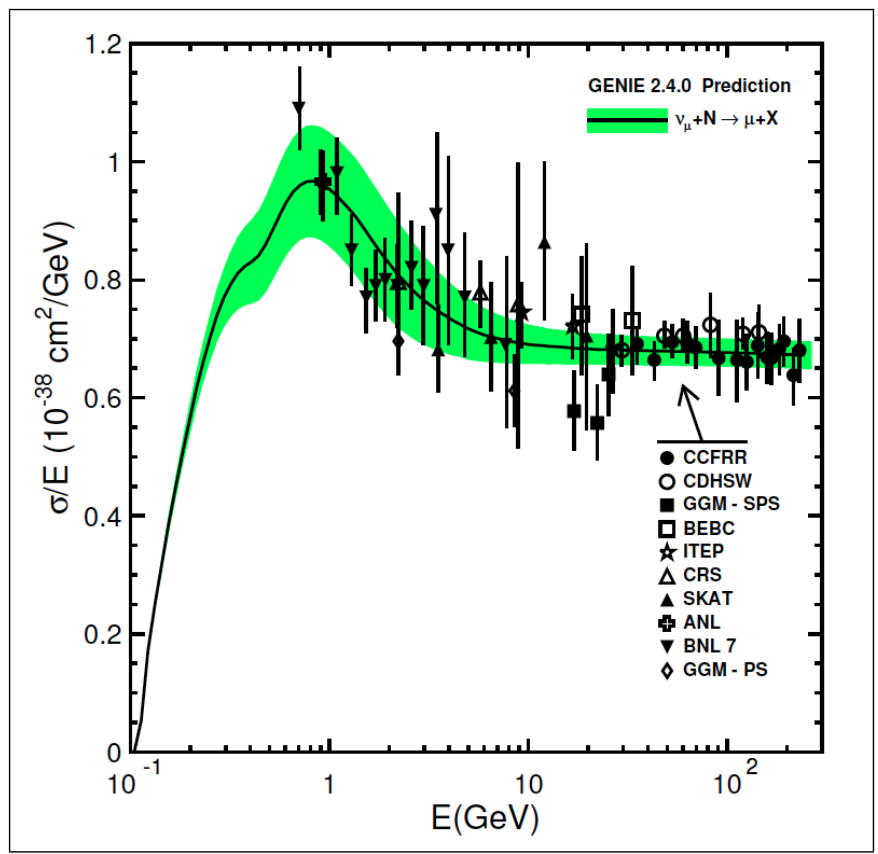

Figure 9.4 GENIE Uncertainty In The Muon Neutrino Cross Section. $\nu_{\mu}$ CC scattering from an isoscalar target. The shaded band indicates the estimated uncertainty on the free nucleon cross section [109]. 
cross sections: [117], [118], [119], [120, 121], [122, 123], [124], [55], and [125].

\subsubsection{Total Reconstruction Efficiency}

The last piece to find the sought flux is the reconstruction efficiency. This efficiency was calculated using the MC simulated sample, and reflects the fraction of events reconstructed given the total number of events available:

$$
\epsilon=\frac{N_{R}}{N_{\mathrm{MC}}},
$$

where $N_{R}$ is the number of reconstructed MC simulated events that passed all the event selection cuts, and $N_{\mathrm{MC}}$ is the number of MC simulated events which had their interaction point inside the VR. The reconstruction efficiency as a function of the neutrino energy, and discriminated per neutrino type and interaction type is presented in figure 9.5.

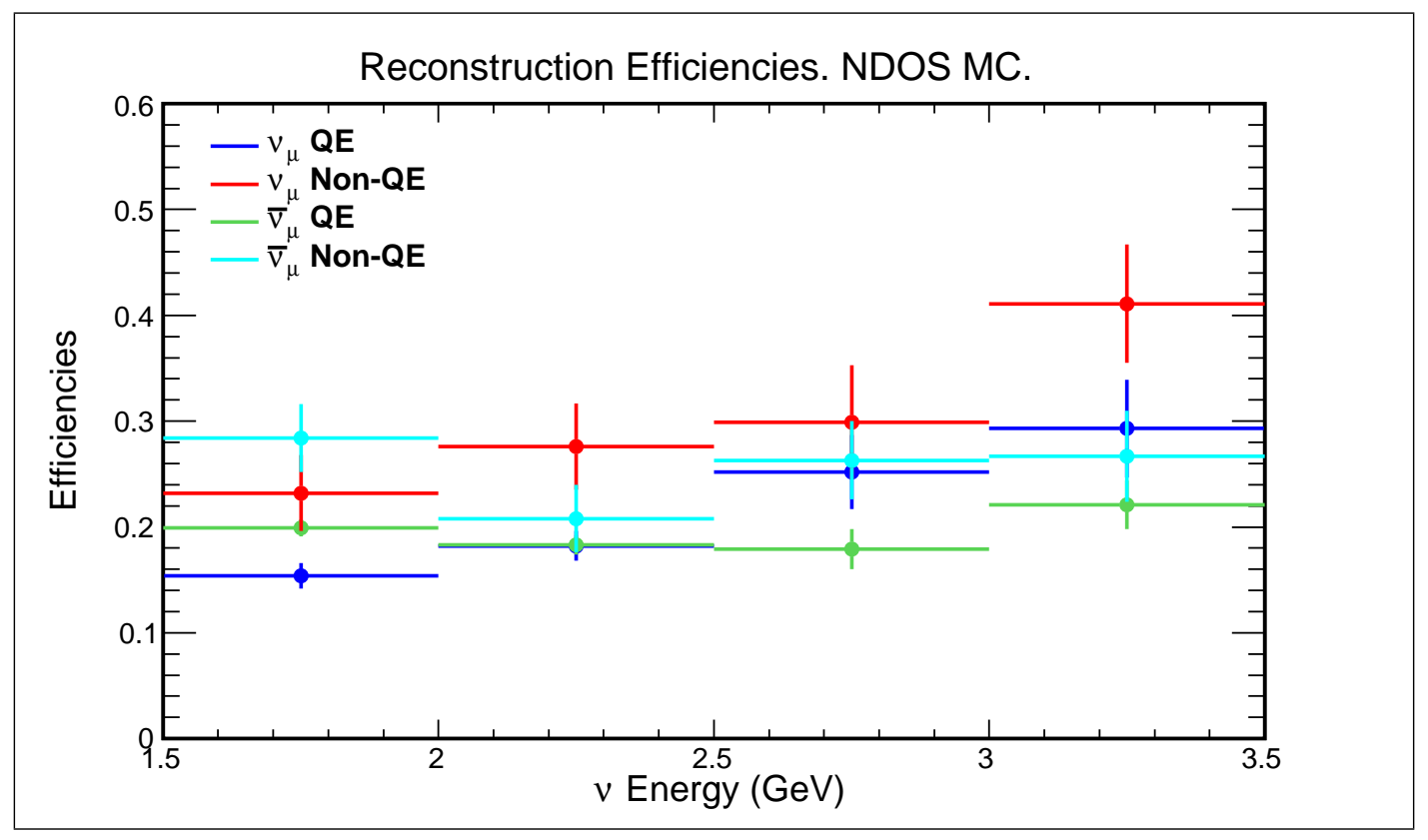

Figure 9.5 Reconstruction Efficiency As A Function Of The Neutrino Energy. Error bars represent the binomial uncertainties. MC simulation. 


\subsection{4 $\nu_{\mu}+\bar{\nu}_{\mu}$ Flux Coming From Charged Kaon Decays}

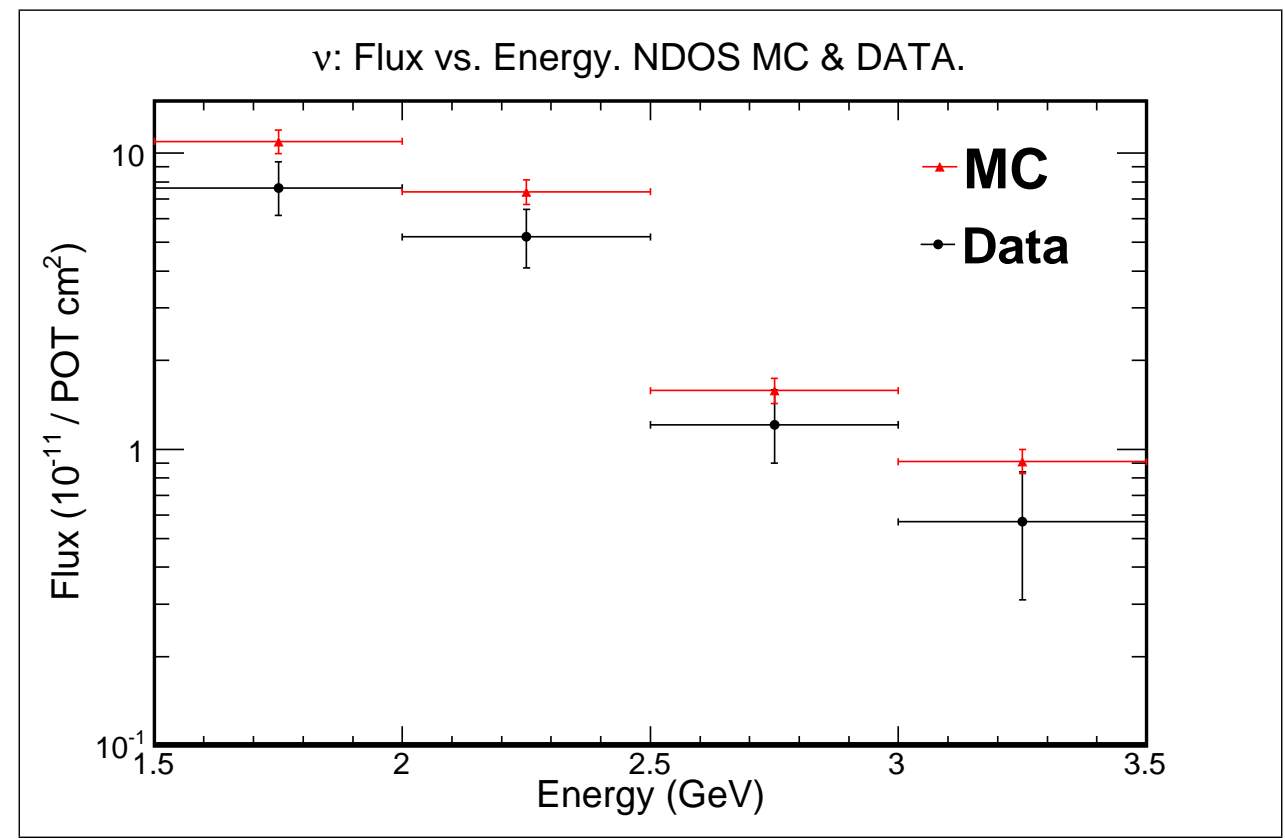

Figure 9.6 Total Flux Of $\nu_{\mu}+\bar{\nu}_{\mu}$ Coming From Charged Kaon Decays. NDOS data (black) and MC simulation (red).

All components of equation (9.4) have been presented so that the flux of $\nu_{\mu}+\bar{\nu}_{\mu}$ produced in charged kaon decays can be determined. The cross sections are given discriminated by interaction type and neutrino type, therefore there are four fluxes:

$$
\Phi_{i}^{\alpha \beta}=\frac{N_{i}^{\alpha \beta}}{\epsilon_{i}^{\alpha \beta} \sigma_{i}^{\alpha \beta} T} ; \alpha=\mathrm{QE}, \text { non-QE; } \beta=\nu_{\mu}, \bar{\nu}_{\mu}
$$

and the total flux is the sum of the four:

$$
\Phi=\sum_{\alpha, \beta} \sum_{i} \Phi_{i}^{\alpha \beta},
$$

where $i$ labels the energy bin. Here, $N$ is the number of neutrino candidate events coming from charged kaon decays $\left(N_{K}\right)$ presented in table 9.2. $T$ is the numbers of target nuclei 
presented in table 9.3. $\sigma$ are the cross sections presented in figure 9.3, and $\epsilon$ are the reconstruction efficiencies presented in figure 9.5. The total flux of $\nu_{\mu}+\bar{\nu}_{\mu}$ coming from charged kaon decays is shown in figure 9.6 in four energy bins of $0.5 \mathrm{GeV}$ size. The uncertainties are the sum in quadrature of statistical and systematic uncertainties. The systematic uncertainties are summarized in figure 9.7. The flux calculated from the NDOS data is systematically lower than that predicted by the NDOS MC simulation (see figure 8.16), within the energy region of interest defined in equation (9.2). The $\chi^{2}$ test for these distributions concludes that $\chi^{2}=9.336$ for ndf $=4$. The ratios between data and MC simulation for the total flux, and also for QE and non-QE interactions, are shown in figure 9.8. The average ratio is about 0.7 .

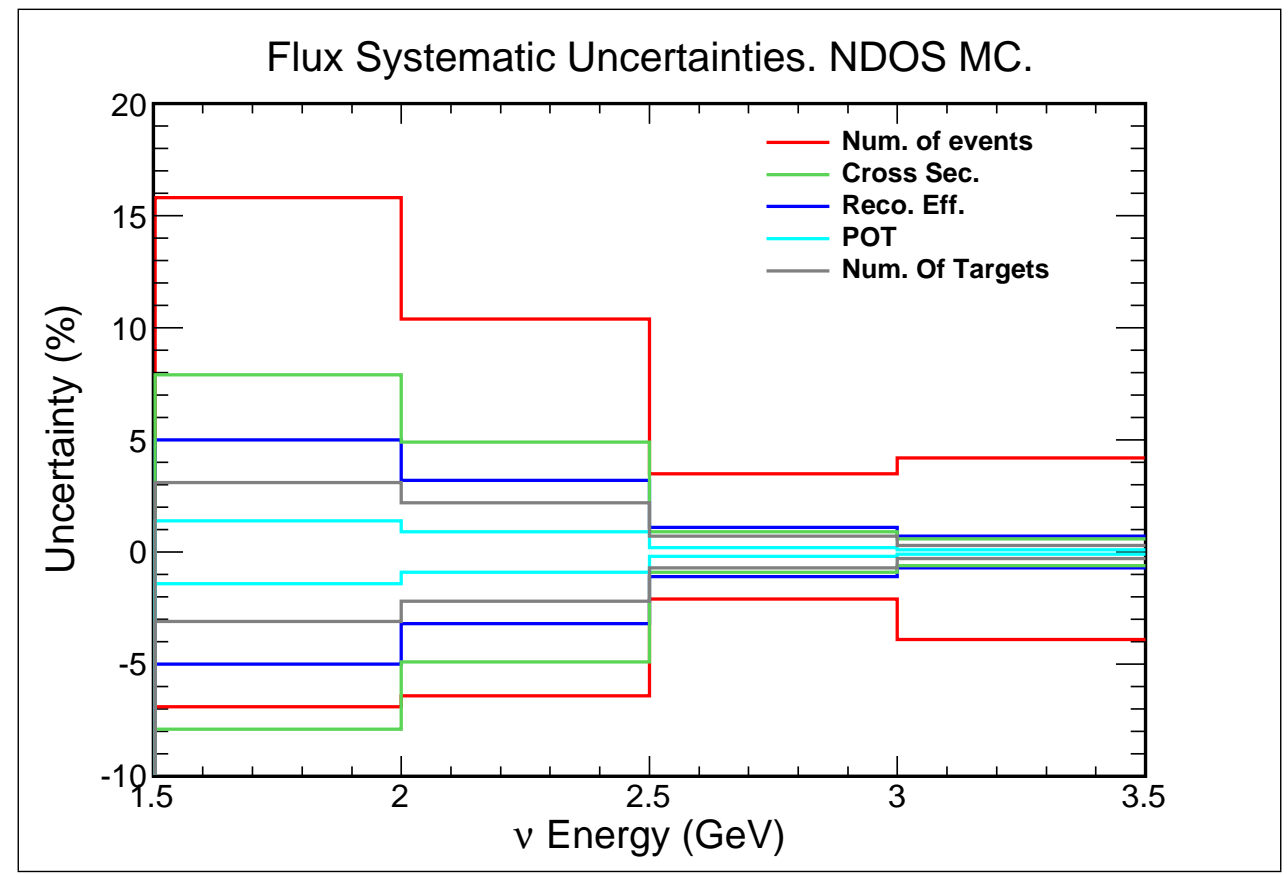

Figure 9.7 Flux Systematic Uncertainties. MC simulation.

Figure 9.9 presents the fluxes discriminated by interaction type. The agreement within error bars of the non-QE fluxes in figure $9.9 \mathrm{~b}$ is better than the one in figure $9.9 \mathrm{a}$ with the 


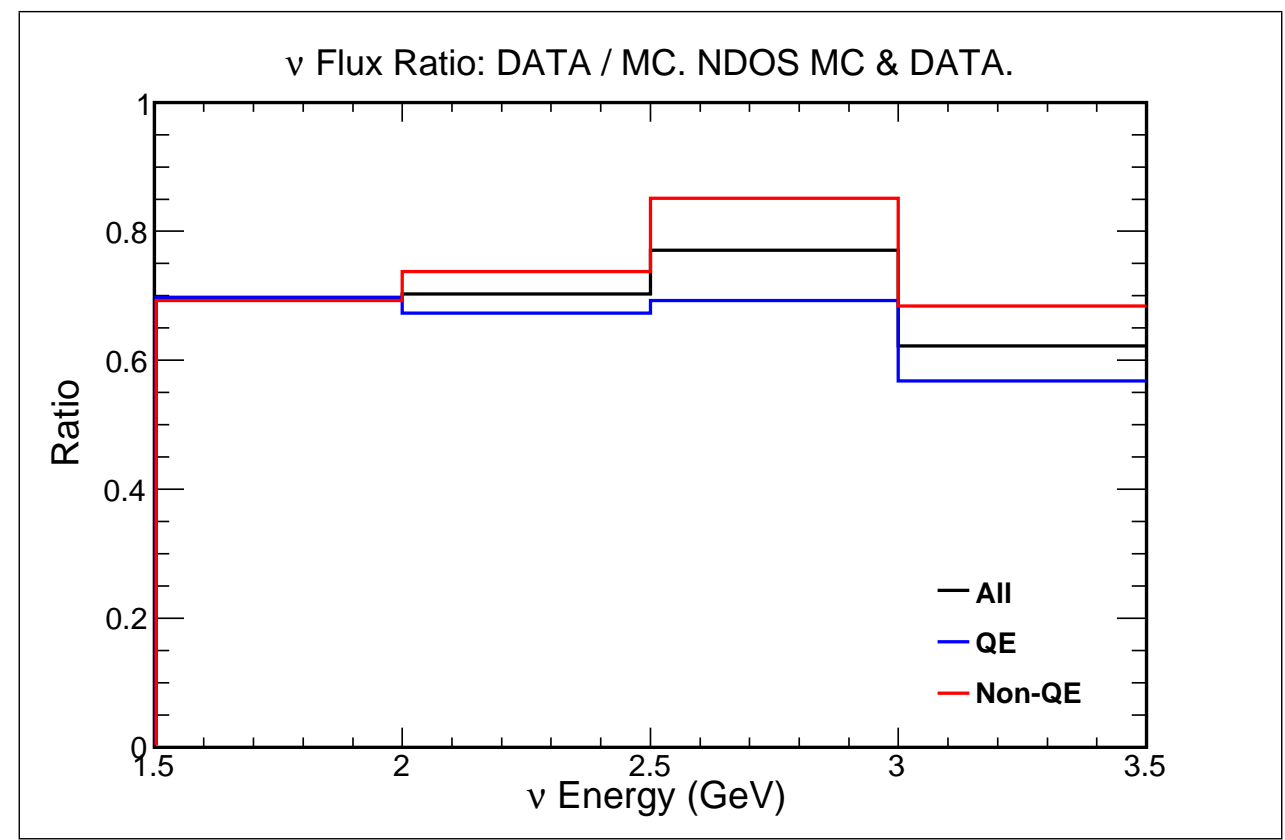

Figure 9.8 Data Over MC Simulated Flux Ratio. Total flux ratio (black), QE flux ratio (blue), and non-QE flux ratio (red) as a function of neutrino energy.
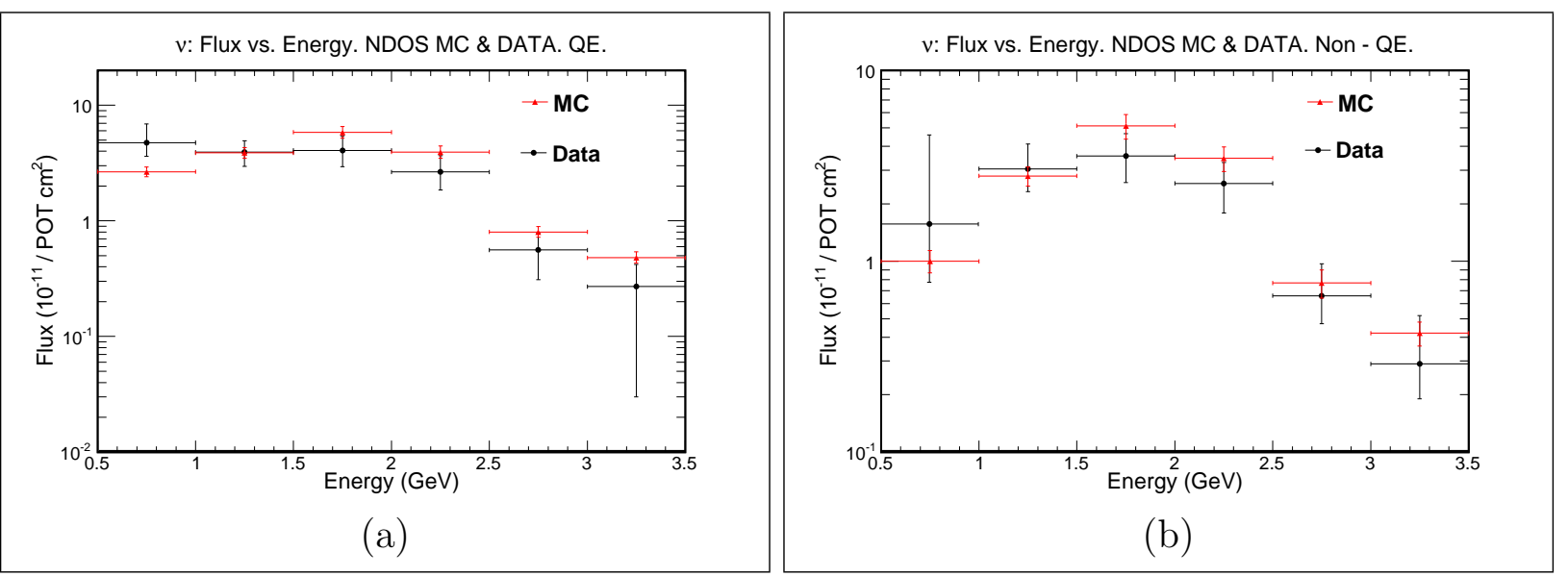

Figure 9.9 Total Flux Of $\nu_{\mu}+\bar{\nu}_{\mu}$ Coming From Charged Kaon Decays Discriminated By Interaction Type. (a) QE and (b) non-QE fluxes. NDOS data (black) and MC simulation (red). 
QE fluxes, though the systematically lower measured flux is present in both interactions. This is corroborated by comparing the two $\chi^{2}$ tests:

$$
\begin{gathered}
\chi_{\mathrm{QE}}^{2}=6.506, \quad \chi_{\mathrm{NQE}}^{2}=3.995, \text { ndf }=4, \\
\frac{\chi_{\mathrm{NQE}}^{2}}{\chi_{\mathrm{QE}}^{2}}=0.614 .
\end{gathered}
$$

Equation (9.10) shows a closer agreement between the non-QE data and the prediction by the $\mathrm{MC}$ simulation than the $\mathrm{QE}$ data does. This result was expected since the non-QE data sample is $60 \%$ larger than the QE one, which is a big difference in statistics, a determinant factor in the analysis. The discrimination between $\mathrm{QE}$ and non-QE flux is useful to check if the two results are consistent with each other, i.e. the flux must be the same, regardless of the interaction, since the difference between them comes from the cross sections. The ratio between the QE over the non-QE fluxes in figure 9.10, is consistent with 1, as expected.

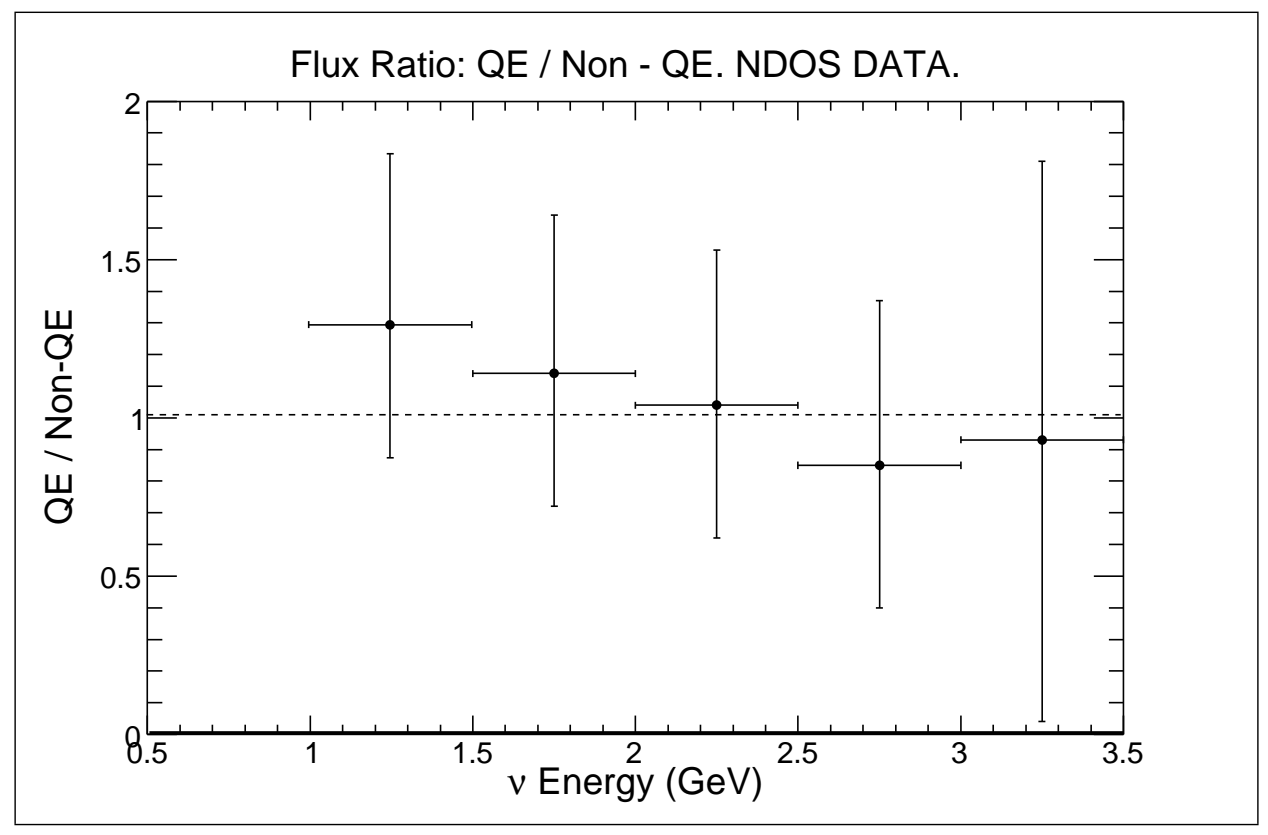

Figure 9.10 QE Over Non-QE Flux Ratio. 
The flux of charged kaons produced at the NuMI target can be constrained comparing the data presented above. From the neutrinos detected with energies stated in equation (9.2), the flux of charged kaons with total average momentum of $6.62 \mathrm{GeV} / \mathrm{c}$ compares to that predicted by the MC simulation as follows:

$$
\begin{aligned}
\Phi_{\text {Data }} & =\left(1.462 \pm 0.154 \mathrm{stat}_{-0.113}^{+0.157} \text { syst }\right) \times 10^{-10} \frac{\nu}{\mathrm{cm}^{2} \mathrm{POT}} \\
\Phi_{\mathrm{MC}} & =\left(2.086_{-0.139}^{+0.141}\right) \times 10^{-10} \frac{\nu}{\mathrm{cm}^{2} \mathrm{POT}} \\
\Phi_{\text {Data }} & =0.701_{-0.094}^{+0.108} \cdot \Phi_{\mathrm{MC}}
\end{aligned}
$$

The observed data and the MC simulated distributions shapes agree, as seen in figures 9.6 and 9.9, though their normalization is not the same, these differ by the amount presented in equation (9.11). This analysis suggests that the neutrino flux of $\nu_{\mu}$ from charged kaon decays obtained from the NDOS data is $30 \%$ lower that is predicted by the MC simulation.

\subsection{Inclusive $\nu_{\mu}$ Charged Current Cross Section Calcu-}

\section{lation}

With the results presented in section 9.1, it becomes interesting to calculate the inclusive $\nu_{\mu} \mathrm{CC}$ cross section using the selected candidates ${ }^{3}\left(N_{\nu_{\mu}}\right)$ presented in table 8.3, and the flux $(\Phi)$ predicted by the MC simulation. With the direct measurement of the number of events done in this analysis it is possible to calculate either the flux, or the cross section, using previous measurements incorporated in the MC simulation of the cross section or the flux, respectively. The excess of predicted events observed in the MC simulation (see

\footnotetext{
${ }^{3}$ The same procedure that led to equation (8.13) applies here for the number of $\nu_{\mu}, N_{\nu_{\mu}}$.
} 
section 8.2) could be the result of overestimating either the flux or the cross section, or a more complicated combination of over and underestimation of the two. A manipulation of equation (9.4) results in the following relation between the cross section and the flux [113]:

$$
\sigma=\sum_{i} \frac{N_{\nu_{\mu}}^{i}}{\epsilon_{i} \Phi_{i} T} .
$$

The two previous direct measurements (see section 2.1.1.4) of the inclusive $\nu_{\mu} \mathrm{CC}$ cross section around $2 \mathrm{GeV}$ (refer to figure 2.4) reported their results per nucleon, therefore the number of targets $(T)$ used in section 9.1.1 needs to be slightly modified from the number of target nuclei to the number of target nucleons. The inclusive cross section per nucleon $\left(\sigma_{N}\right)$ is the sum of the $\mathrm{QE}$ cross section per nucleon $\left(\sigma_{\mathrm{QE}}\right)$ plus the non-QE cross section per nucleon $\left(\sigma_{\mathrm{nQE}}\right)$ :

$$
\sigma_{N}=\sigma_{\mathrm{QE}}+\sigma_{\mathrm{nQE}} .
$$

The number of targets in the TR is:

$$
\begin{aligned}
& T=1.723 \times 10^{30} \text { neutrons, or } \\
& T=3.854 \times 10^{30} \text { nucleons. }
\end{aligned}
$$

The FLUKA $\nu_{\mu}$ flux prediction $\left(\Phi_{i}\right)$, that is embedded in the MC simulated sample, is shown as a function of neutrino energy in figure 9.11. The same FLUKA simulation was used by the MiniBoone collaboration on their measurement of $\nu_{\mu}$ and $\nu_{e}$ events [126]. The MiniBoone detector is also located at $110 \mathrm{mrad}$ off the NuMI beam axis. Their measurements have a $9 \%$ systematic uncertainty, on the NuMI flux, in the energy range between $0.9 \mathrm{GeV}$ and $3.0 \mathrm{GeV}$. In this analysis the systematic uncertainty related to the FLUKA flux 


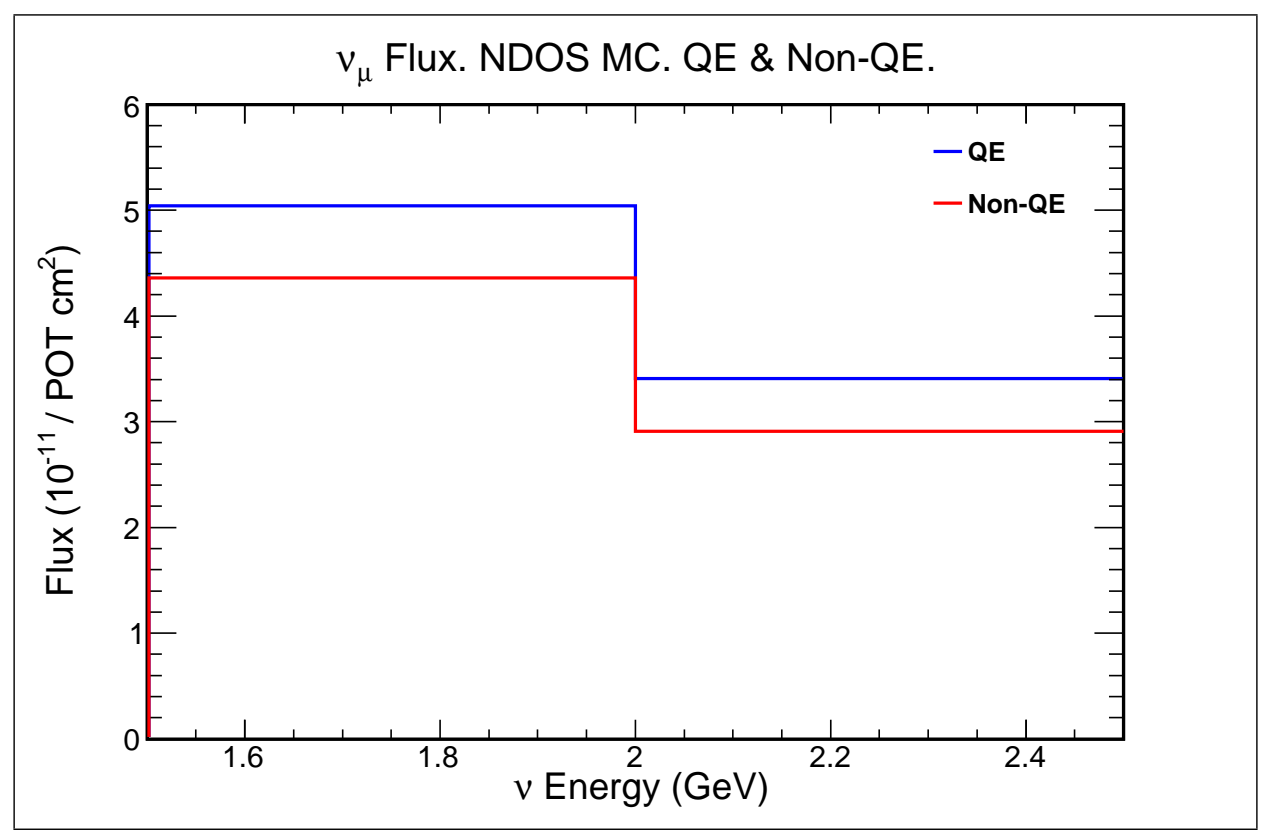

Figure 9.11 Muon Neutrino MC Simulated Flux Prediction.

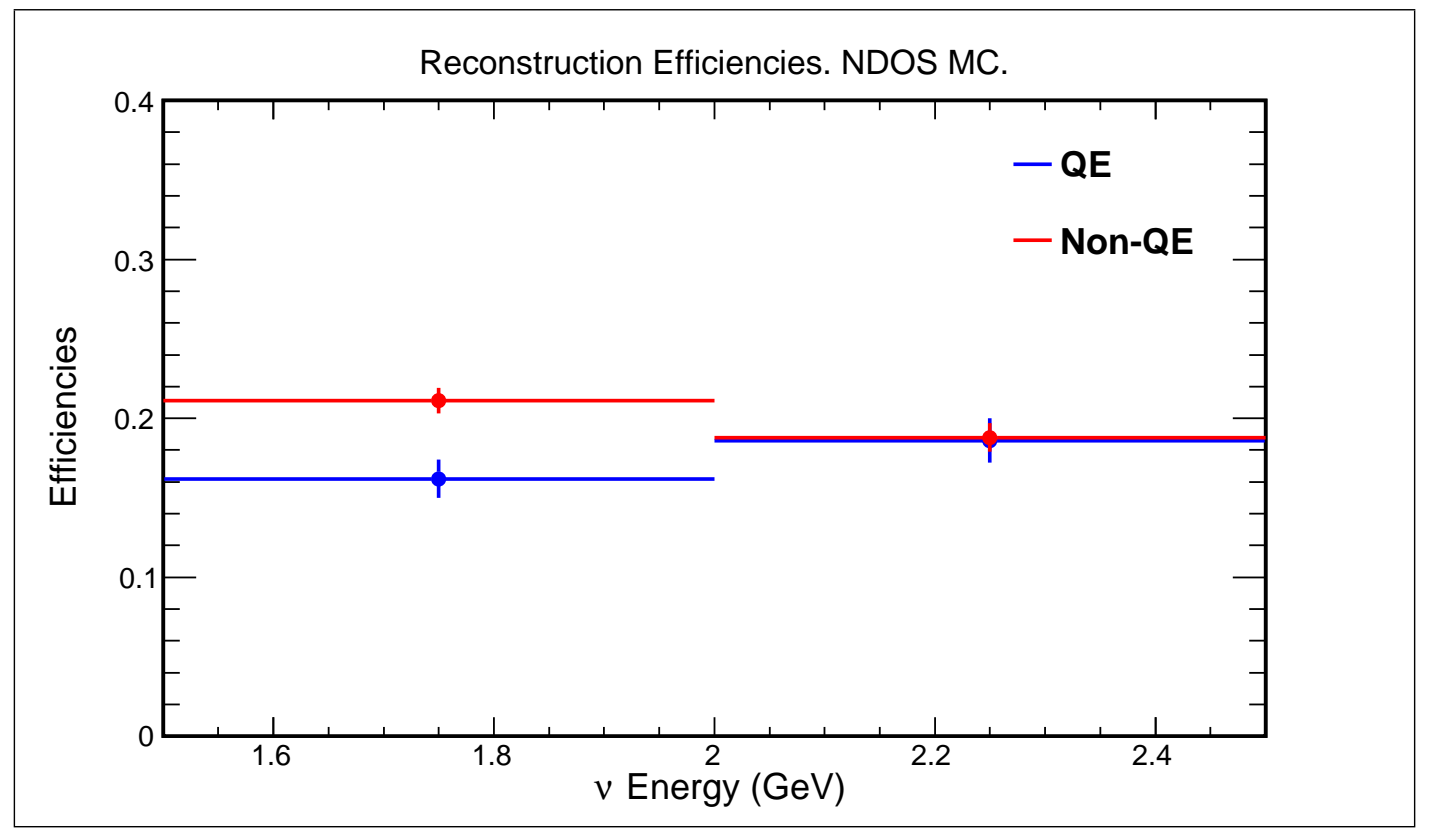

Figure 9.12 Reconstruction Efficiency As A Function Of The Neutrino Energy. Error bars represent the binomial uncertainties. MC simulation. 
prediction is assumed to be $10 \%$ based on the MiniBoone estimation.

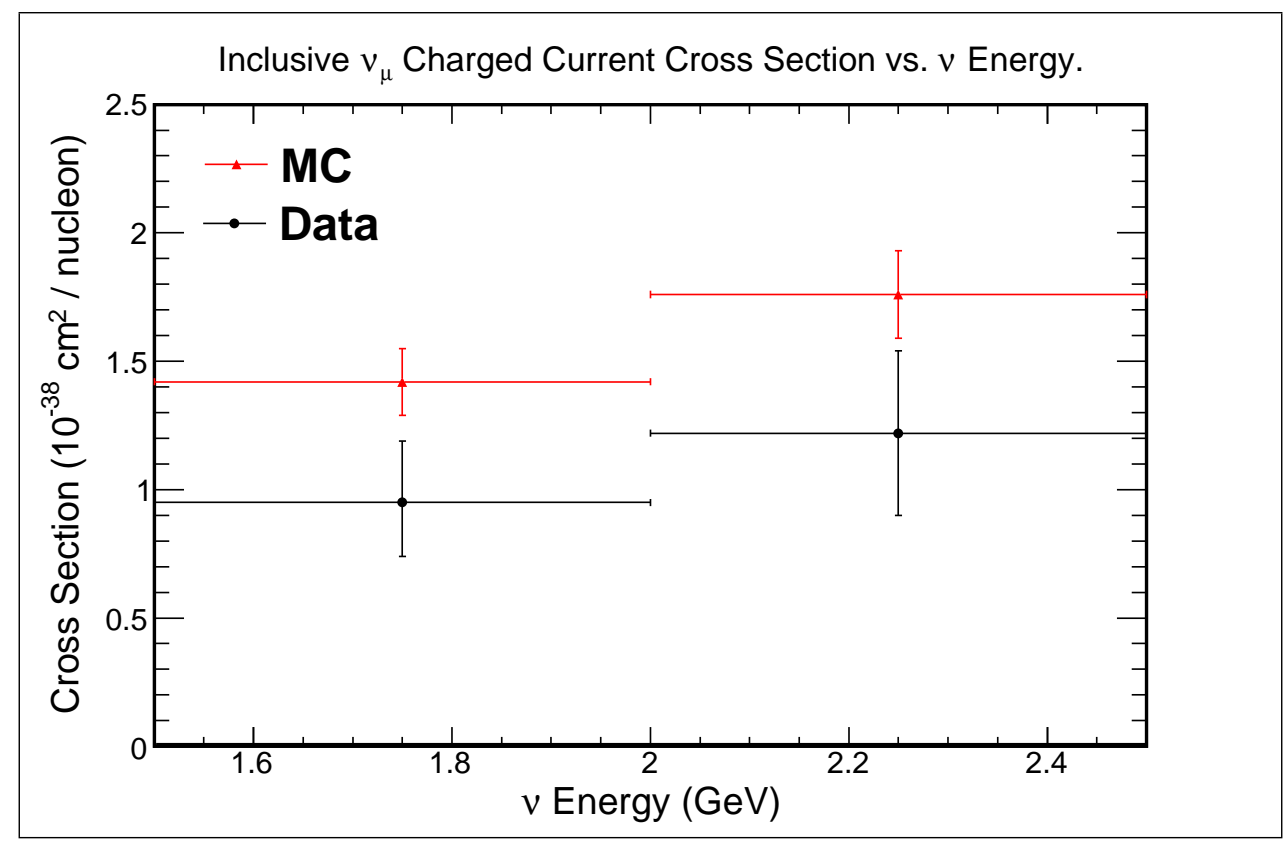

Figure 9.13 Inclusive Muon Neutrino Charged Current Cross Section Per Nucleon On A Carbon Target. NDOS data (black) and MC simulation (red).

The last pieces to calculate the inclusive $\nu_{\mu} \mathrm{CC}$ cross section are the reconstruction efficiencies (defined in equation (9.7)) presented in figure 9.12. Following a procedure similar to that shown in section 9.1.4 leads to the inclusive $\nu_{\mu}$ CC cross section per nucleon on a carbon target ${ }^{4}$. The cross section is presented in two energy bins of $0.5 \mathrm{GeV}$, as seen in figure 9.13. The uncertainties are the sum in quadrature of statistical and systematic uncertainties. The systematic uncertainties are summarized in figure 9.14. The cross section calculated using the NDOS data is systematically lower than that embedded in the NDOS MC simulation. The $\chi^{2}$ test for these distributions concludes that $\chi^{2}=6.803$ for ndf $=2$. The ratio between the data and MC simulated cross sections is shown in figure 9.15, only the error bars in the second bin are consistent with one, which is shown as a dashed line.

\footnotetext{
${ }^{4}$ Refer to table 9.3 .
} 


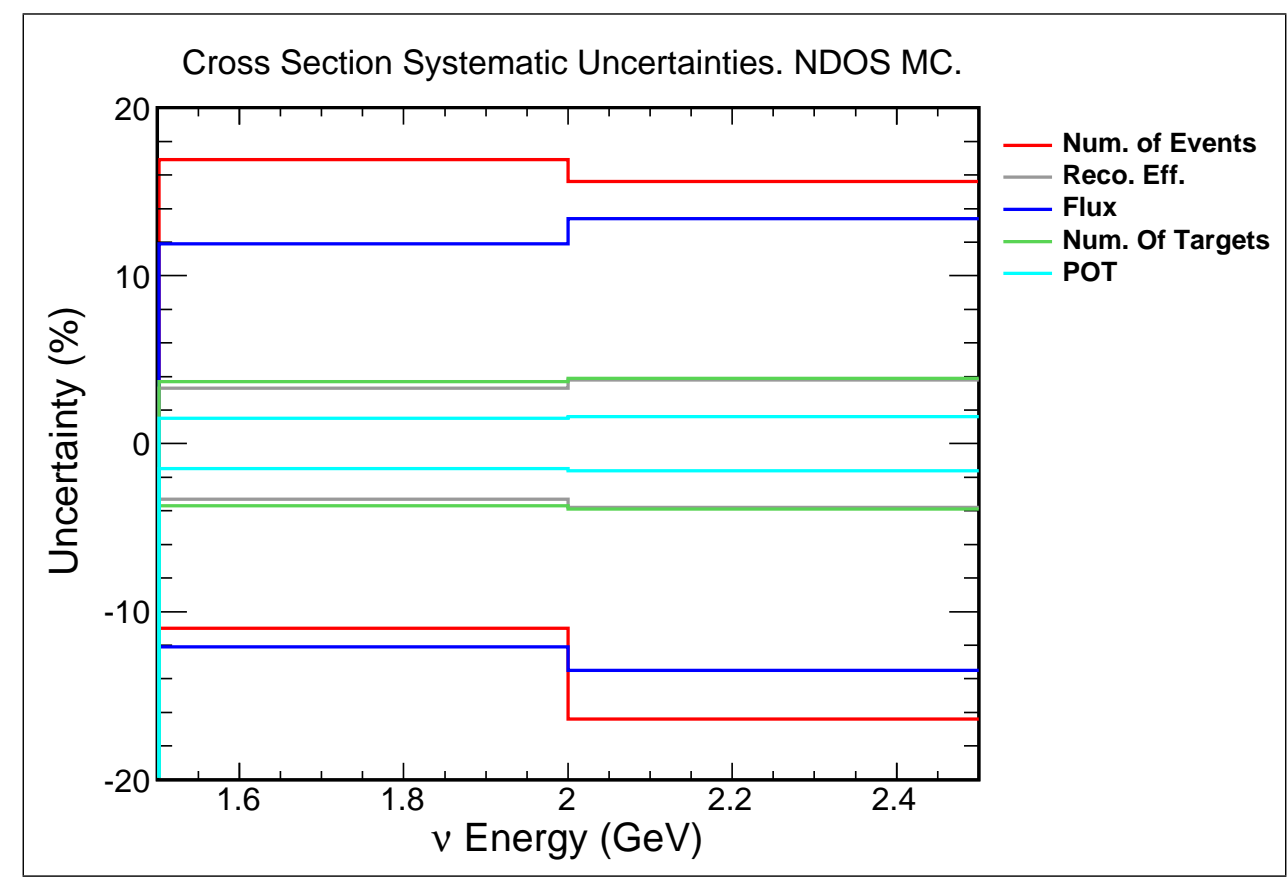

Figure 9.14 Cross Section Systematic Uncertainties. MC simulation.

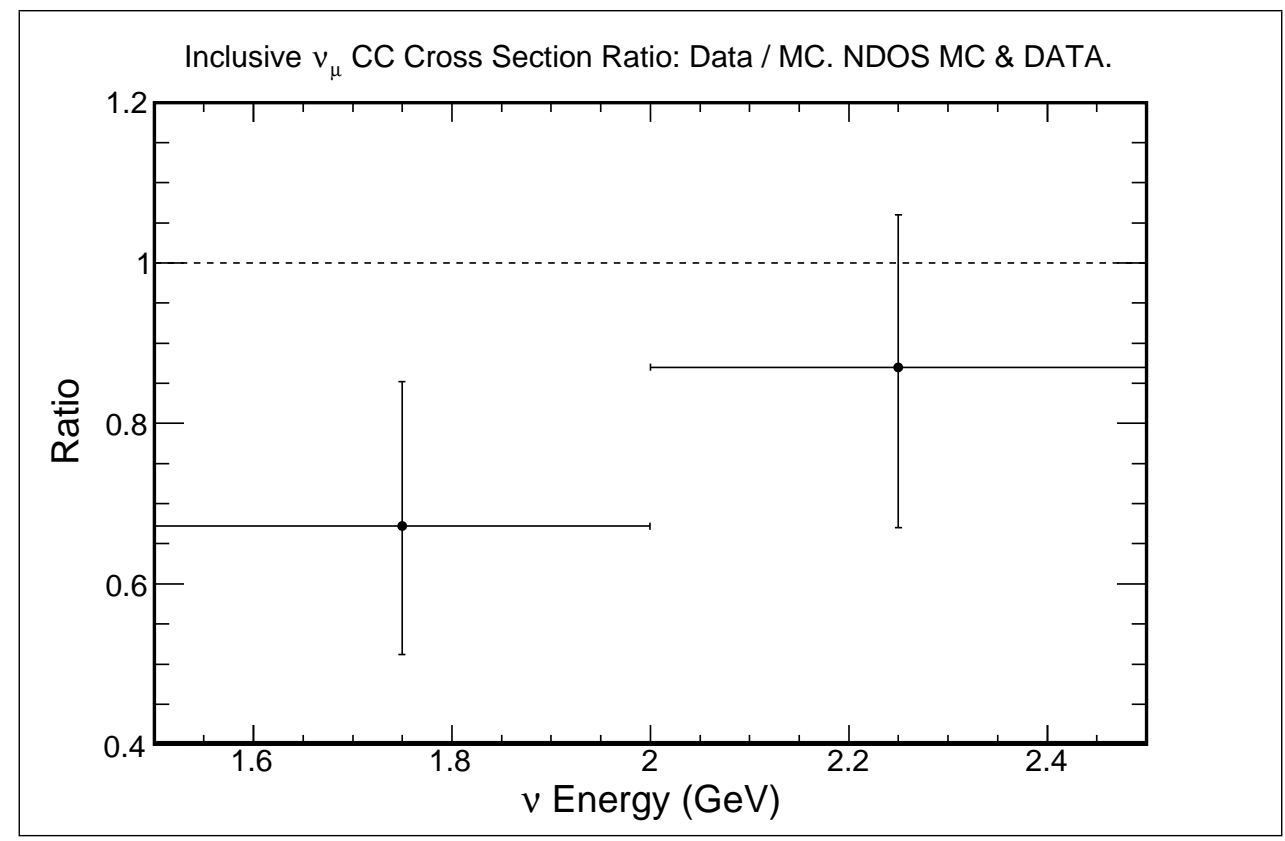

Figure 9.15 Data Over MC Simulated Muon Neutrino Charged Current Cross Section Ratio. 


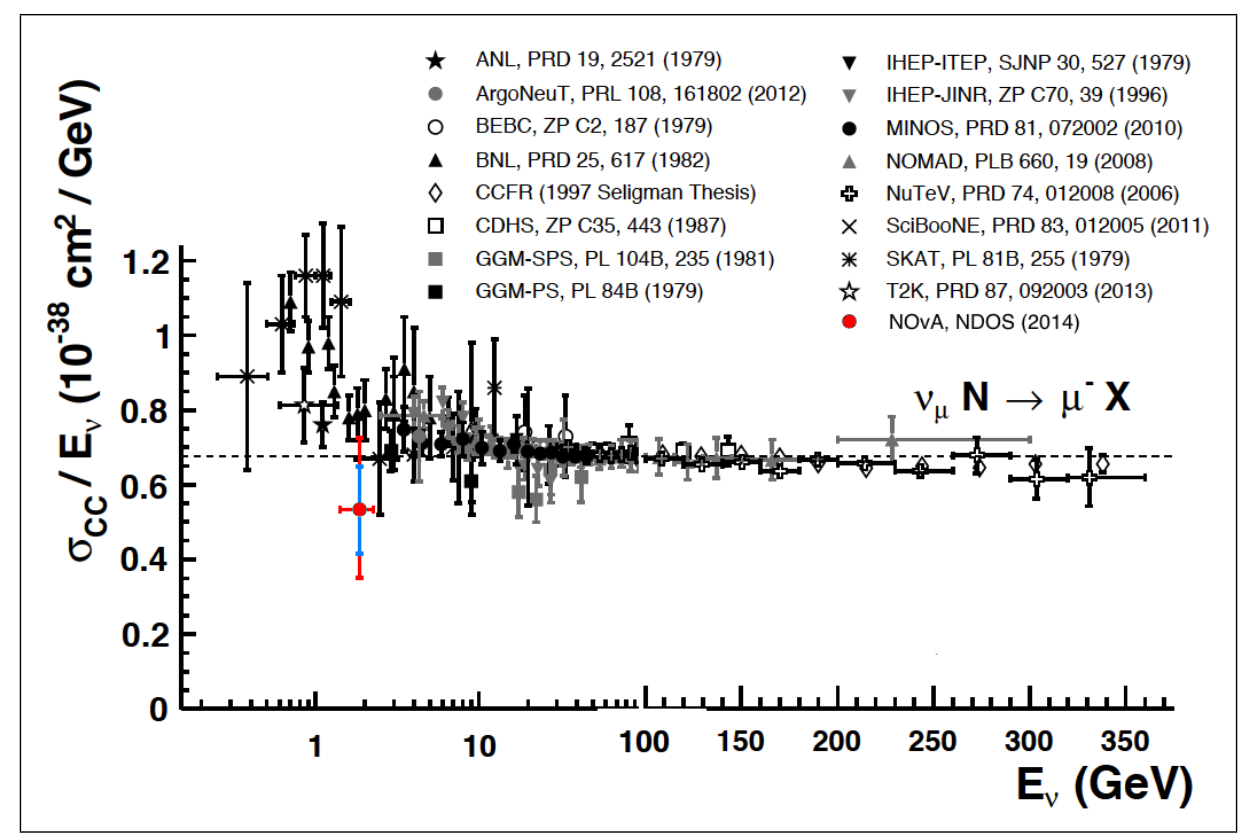

Figure 9.16 Measurements Of Muon Neutrino Charged Current Inclusive Scattering Cross Sections Divided By Neutrino Energy. All the results are cited in the figure. Vertical blue (red) error bars represent statistical (combined statistical and systematic) errors. The NDOS data point is inserted into a figure taken from [44].

The inclusive $\nu_{\mu} \mathrm{CC}$ cross section per nucleon at an average neutrino energy of $1.97 \mathrm{GeV}$ compares to that embedded in the MC simulation as follows:

$$
\begin{aligned}
\sigma_{\text {Data }} & =\left(1.085 \pm 0.219 \mathrm{stat}_{-0.383}^{+0.330} \mathrm{syst}\right) \times 10^{-38} \mathrm{~cm}^{2}, \\
\sigma_{\mathrm{MC}} & =\left(1.588_{-0.210}^{+0.216}\right) \times 10^{-38} \mathrm{~cm}^{2}, \\
\sigma_{\text {Data }} & =0.683_{-0.257}^{+0.266} \cdot \sigma_{\mathrm{MC}} .
\end{aligned}
$$

The comparison of the measured cross section with previous measurements is presented in figure 9.16, which summarizes the measurements of $\nu_{\mu} \mathrm{CC}$ inclusive scattering cross sections divided by the neutrino energy as a function of neutrino energy. Note the transition between logarithmic and linear scales occurring at $100 \mathrm{GeV}$. The red error bars are the sum in quadrature of the statistical and systematic uncertainties. The blue error bars are the 
statistical uncertainties alone. The measured cross section agrees, within error bars, with the two previous results $[55,56]$ mentioned above, and lies below the trend set by the collection of all the results shown. The average cross section over neutrino energy of the wide range of energies shown is represented by the horizontal dashed line.

\subsection{Final Remarks}

The two results presented in equations $(9.11,9.15)$ are the possible consequences of the difference of about $1 / 3$ seen in section 8.2 between the selected NDOS $\nu_{\mu}+\bar{\nu}_{\mu}$ CC candidates and the prediction done by MC simulation. The conclusion is that the FLUKA flux needs to be adjusted, based on the two NDOS measurements available ${ }^{5}$, by $30 \%$ since the inclusive $\nu_{\mu} \mathrm{CC}$ cross section (over neutrino energy) embedded in the MC simulation is consistent with the dashed line in figure 9.16 at $2 \mathrm{GeV}$.

NDOS was designed to serve as a testing instrument that would provide the $\mathrm{NO} \nu \mathrm{A}$ collaboration with a proof of principle for the concepts and technologies developed for the experiment. Measuring the flux of $\nu_{\mu}+\bar{\nu}_{\mu}$ coming from charged kaon decays was a worth pursuing result since those neutrinos show a clean peak around $2 \mathrm{GeV}$, and the flux of charged kaons had not been measured at the angle where NDOS sits. A 30\% excess of events predicted by the MC simulation is now reported by two different analyses of the NDOS data, which should be taken into account by the neutrino oscillations community. $\mathrm{NO} \nu \mathrm{A}$ can verify the results presented in this analysis with its near detector underground which is taking data now.

\footnotetext{
${ }^{5}$ The result of this analysis and that from [22].
} 
APPENDICES 


\section{Appendix A}

\section{Mass Terms In The Weak Interaction}

All leptons participate in the weak interaction. However it is the only interaction in the Standard Model where neutrinos are involved ${ }^{1}$. Leptons and quarks enter in the weak interaction as left-handed weak-isospin doublets ${ }^{2}$ :

$$
L \equiv\left(\begin{array}{l}
\nu \\
l
\end{array}\right), \quad q \equiv\left(\begin{array}{l}
u \\
d
\end{array}\right),
$$

where the left-handed states are:

$$
\begin{aligned}
\nu_{L} & =\frac{1}{2}\left(1-\gamma_{5}\right) \nu, \quad l_{L}=\frac{1}{2}\left(1-\gamma_{5}\right) l, \\
u_{L}=\frac{1}{2}\left(1-\gamma_{5}\right) u, & d_{L}=\frac{1}{2}\left(1-\gamma_{5}\right) d .
\end{aligned}
$$

Here: $u=u, c, t ; d=d, s, b ; l=e, \mu, \tau ;$ and $\nu=\nu_{e}, \nu_{\mu}, \nu_{\tau}$. There is no experimental evidence of the existence of right-handed neutrinos ${ }^{3}$, and since these only interact with other

\footnotetext{
${ }^{1}$ Neutrinos do not participate in the electromagnetic interaction at the tree level. If neutrinos are Dirac particles these can have a magnetic moment, and therefore participate in the electromagnetic interaction through one-loop diagrams. The transition moment, which is relevant to $\nu_{i} \rightarrow \nu_{j}+\gamma(i \neq j)$, may exist for both Dirac and Majorana neutrinos [64]. For a detailed calculation of the one-loop diagram see [127, 128, 129].

${ }^{2}$ The doublet in equation (A.1) is a general case which works for all three families.

${ }^{3}$ Right-handed neutrinos have chirality eigenvalue: +1 . Chirality is an intrinsic property, independent of the reference frame of the observer. Since neutrinos are massive, they can have both eigenvalues of helicity: \pm 1 , which is a property that depends on the reference frame of the observer. Chirality and helicity are only equivalent for massless particles [130].
} 
particles through the weak interaction ${ }^{4}$, the Standard Model excludes them. Right-handed charged leptons and right-handed quarks enter the Standard Model weak interaction as weak-isospin singlets given by:

$$
\begin{gathered}
l_{R}=\frac{1}{2}\left(1+\gamma_{5}\right) l \\
u_{R}=\frac{1}{2}\left(1+\gamma_{5}\right) u, \quad d_{R}=\frac{1}{2}\left(1+\gamma_{5}\right) d .
\end{gathered}
$$

Weak-isospin and weak hypercharge are related by the Gell-Mann-Nishijima [131, 132] relation:

$$
Q=I_{3}+\frac{Y}{2}
$$

where $Q$ is the electric charge, $I_{3}$ is the third component of the weak-isospin, and $Y$ is the weak hypercharge. Weak-isospin can only take two values $I_{3}= \pm 1 / 2$, thus:

$$
Y_{L}=-1, \quad Y_{R}=-2
$$

With the commutator:

$$
\left[I_{3}, Y\right]=0
$$

the two quantities are commuting observables, and the product of the group transformations generated by $I$ and $Y$ is the gauge group $S U(2)_{L} \otimes U(1)_{Y}$ of a gauge theory. Four massless bosons and six massless leptons are the result of these constructions [130]. The Lagrangian of this group is:

$$
\mathcal{L}_{G}=\mathcal{L}_{g}+\mathcal{L}_{\phi}+\mathcal{L}_{f}+\mathcal{L}_{h}
$$

\footnotetext{
${ }^{4}$ The weak interaction only operates on left-handed states [27].
} 
where $\mathcal{L}_{g}$ is the gauge Lagrangian, $\mathcal{L}_{\phi}$ is the scalar Lagrangian, $\mathcal{L}_{f}$ is the fermionic Lagrangian, and $\mathcal{L}_{h}$ is the Yukaga Lagrangian. The gauge part of the Lagrangian is:

$$
\mathcal{L}_{g}=-\frac{1}{4} W_{\mu \nu}^{i} W_{i}^{\mu \nu}-\frac{1}{4} B_{\mu \nu} B^{\mu \nu}
$$

where $W_{\mu \nu}^{i}$ is the $S U(2)$ gauge field that has three and four-point self-interactions, and $B_{\mu \nu}$ is the $U(1)$ gauge field associated with $Y$, which has no self-interaction [130].

The scalar part of the Lagrangian is:

$$
\mathcal{L}_{\phi}=\left(D^{\mu} \phi\right)^{\dagger} D^{\mu} \phi-V(\phi)
$$

where $D^{\mu}$ is a covariant derivative [133], and $V(\phi)$ is a scalar potential. In order to generate the masses of the weak bosons and all the charged leptons, a doublet of scalar fields $(\phi)$ defined as:

$$
\phi \equiv\left(\begin{array}{c}
\phi^{+} \\
\phi^{0}
\end{array}\right)
$$

is introduced. The known masses are generated through a spontaneous symmetry breaking in the potential $V(\phi)$ of the field. This transforms as a $S U(2)_{L}$ doublet which implies: $Y_{\phi}=1$. The invariance and renormalizability [134] of the group restrict $V(\phi)$ to the form:

$$
V(\phi)=\mu^{2} \phi^{\dagger} \phi+|\lambda|\left(\phi^{\dagger} \phi\right)^{2}
$$

where $\mu$ and $\lambda$ are parameters. For $\mu>0$ the potential is quadratic with its only minimum at zero, as shown in figure A.1a, and the vacuum is empty. An exact symmetry is characterized by two conditions: the Lagrangian density is invariant under the symmetry, and the physical 
vacuum is invariant under the symmetry transformations [130]. A model with an exact symmetry contains a degenerate set of massless particles. The Standard Model has symmetry breaking to account for the observed masses in the model. The case for $\mu<0$, in equation (A.11), is shown in figure A.1b. There is a spontaneous symmetry breaking since the scalar field takes one of all the possible minima in the potential.
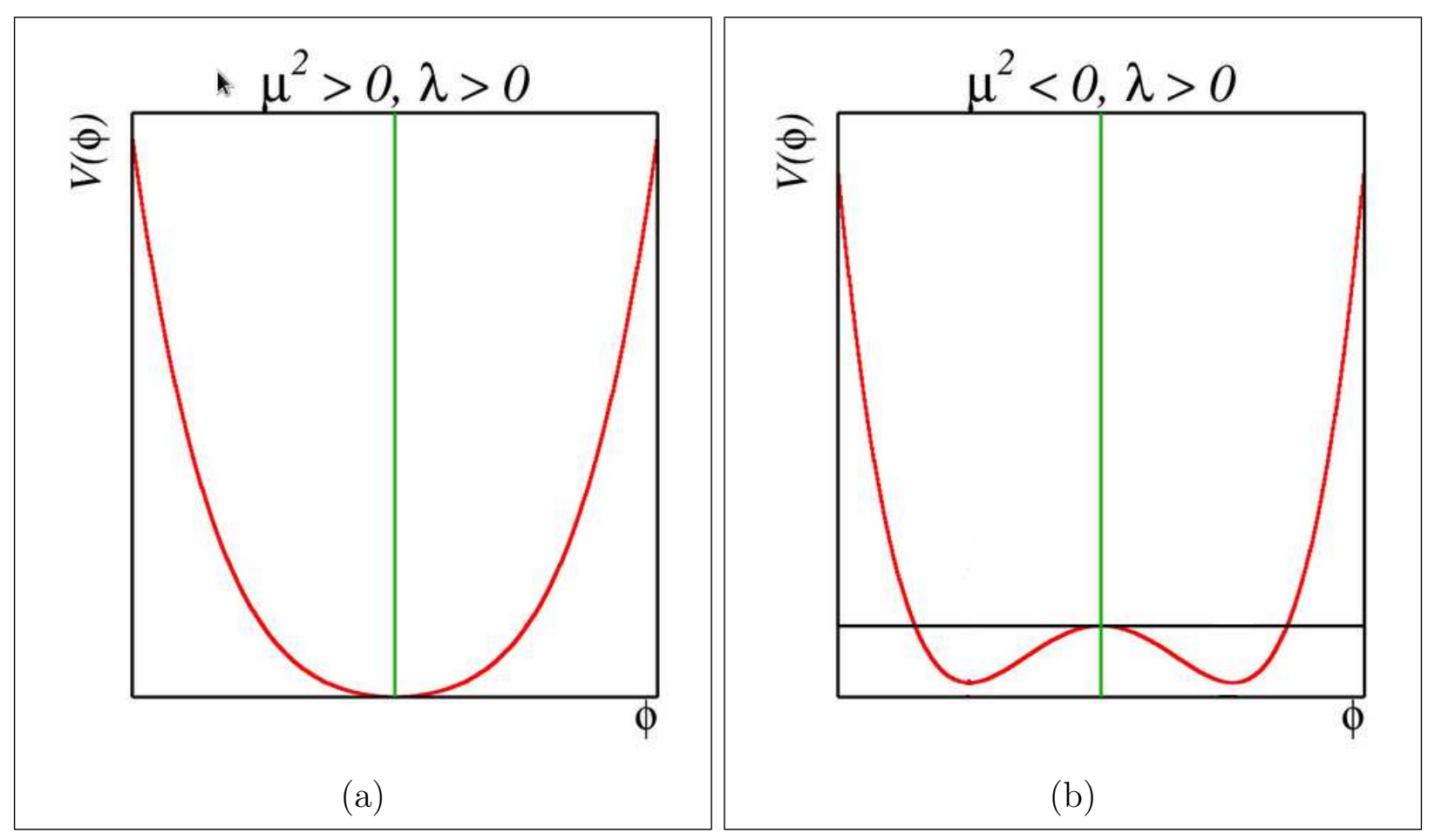

Figure A.1 Scalar Potential. (a) $\mu>0$, and (b) $\mu<0$ [135].

The fermionic part of the Lagrangian is:

$$
\mathcal{L}_{f}=\sum_{k=1}^{3}\left(\bar{q}_{L}^{k} \imath \not D q_{L}^{k}+\bar{l}_{L}^{k} \imath \not D l_{L}^{k}+\bar{u}_{R}^{k} \imath \not D u_{L}^{k}+\bar{d}_{R}^{k} \imath \not D q_{R}^{k}+\bar{l}_{R}^{k} \imath \not D l_{R}^{k}\right),
$$

where $k$ denotes the family, and all fields are weak eigenstates [130]. 
The Yukawa part of the Lagrangian is:

$$
\mathcal{L}_{h}=-\sum_{k, n=1}^{3}\left[\Gamma_{k n}^{u} \bar{u}_{R}^{n}\left(\imath \tau^{2} \phi^{\dagger} q_{L}^{k}\right)+\Gamma_{k n}^{d} \bar{d}_{R}^{n}\left(\imath \tau^{2} \phi^{\dagger} q_{L}^{k}\right)+\Gamma_{k n}^{l} \bar{l}_{R}^{n}\left(\imath \tau^{2} \phi^{\dagger} l_{L}^{k}\right)\right]+\text { h.c. }
$$

where the matrices $\Gamma_{k n}$ describe the Yukawa couplings between the scalar doublet and the various flavors $k$ and $n$ of quarks and leptons [130].

The vacuum expectation values of the various complex scalar fields are the components $\phi_{i}$ of a complex vector $v$ :

$$
v=\langle 0|\phi| 0\rangle
$$

Without any loss of generality the four axes of this space can be chosen so that:

$$
\left\langle 0\left|\phi_{i}\right| 0\right\rangle=0 \text {, for } i=1,2 \text {, and } 4 \text {, and }\left\langle 0\left|\phi_{3}\right| 0\right\rangle=v \text {. }
$$

Here $v$ is determined by writing the scalar potential: $V(\phi) \rightarrow V(v)$, and choosing $v$ such that $V$ is minimized [136]. The quantum theory is obtained by considering the fluctuations around this minimum: $\phi=v+\phi^{\prime}$. Using equation (A.15), the potential in equation (A.11) becomes:

$$
V(\phi) \rightarrow V(v)=\frac{1}{2} \mu^{2} v^{2}+\frac{1}{4}|\lambda| v^{4}
$$

For $\mu>0$ in figure A.1a, the minimum of the potential occurs at $v=0$, and no symmetry breaking occurs. For $\mu<0$ in figure A.1b, the minimum is obtained at a non-zero value of $v$ :

$$
\frac{d V(v)}{d v}=v\left(\mu^{2}+|\lambda| v^{2}\right)=0
$$


which has solution ${ }^{5}$ :

$$
v=\left(-\frac{\mu^{2}}{|\lambda|}\right)^{1 / 2}
$$

With this result, the kinetic part of the scalar Lagrangian in equation (A.9) becomes:

$$
\left(D_{\mu} \phi\right)^{\dagger} D^{\mu} \phi=\frac{1}{2}(0 v)\left[\frac{g}{2} \tau^{i} W_{\mu}^{i}+\frac{g^{\prime}}{2} B_{\mu}\right]^{2}\left(\begin{array}{l}
0 \\
v
\end{array}\right)+\cdots
$$

where $g$ and $g^{\prime}$ are coupling constants of the $S U(2)$ and $U(1)$ gauge groups. After some algebraic manipulation [138], important mass terms are identified ${ }^{6}$ :

$$
\begin{aligned}
1: & \frac{g^{2} v^{2}}{8}\left(W^{1}-\imath W^{2}\right)^{\mu}\left(W^{1}+\imath W^{2}\right)_{\mu}, \\
2: & \frac{\left(g^{2}+g^{\prime 2}\right) v^{2}}{8}\left(\frac{g W^{3}}{\sqrt{g^{2}+g^{\prime 2}}}-\frac{g^{\prime} B}{\sqrt{g^{2}+g^{\prime 2}}}\right)^{\mu}\left(\frac{g W^{3}}{\sqrt{g^{2}+g^{\prime 2}}} W^{3}-\frac{g^{\prime} B}{\sqrt{g^{2}+g^{\prime 2}}}\right)_{\mu} .
\end{aligned}
$$

The masses of the two weak interaction bosons are:

$$
M_{W}=\frac{g v}{2}, \quad M_{Z}=\frac{v \sqrt{g^{2}+g^{\prime 2}}}{2}
$$

and the weak mixing angle $\left(\theta_{W}\right)$ fulfills:

$$
\cos \theta_{W}=\frac{g}{\sqrt{g^{2}+g^{\prime 2}}}, \quad \sin \theta_{W}=\frac{g^{\prime}}{\sqrt{g^{2}+g^{\prime 2}}}
$$

1 and 2 in equation (A.20) can be written as:

$$
1: M_{W}^{2} W^{+\mu} W_{\mu}^{-}, 2: \frac{M_{Z}^{2}}{2} Z^{\mu} Z_{\mu}
$$

${ }^{5}$ The case for $\mu=0$ requires loop corrections, but the symmetry is again spontaneously broken [137].

${ }^{6}$ These are mass terms since the fields couple to themselves. 
where:

$$
W^{ \pm}=\frac{1}{\sqrt{2}}\left(W^{1} \mp \imath W^{2}\right), \text { and } Z=\cos \theta_{W} W^{3}-\sin \theta_{W} B
$$

are the weak interaction bosons. From the form of the covariant derivative, the electron's charge is [133]:

$$
e=\frac{g g^{\prime}}{\sqrt{g^{2}+g^{\prime 2}}}
$$

which allows to estimate the weak interaction bosons' masses. Notice that the term:

$$
A^{\mu} A_{\mu}=\left(\sin \theta_{W} W^{3}+\cos \theta_{W} B\right)^{\mu}\left(\sin \theta_{W} W^{3}+\cos \theta_{W} B\right)_{\mu},
$$

is multiplied by the zeroes in equation (A.19), thus the photon field $(A)$, given by:

$$
A=\sin \theta_{W} W^{3}+\cos \theta_{W} B
$$

is massless. The coefficients $M_{W}$ and $M_{Z}$ (in equation (A.23)) depend on $v$, which is ${ }^{7}$ the vacuum expectation value of the scalar field. This scalar field is known as the Higgs field [139]. The spontaneous symmetry breaking that results in $v \neq 0$ produces three massive weak interaction messengers and a massless electromagnetic interaction one [130]; unifying the weak and electromagnetic interaction into one elegant electroweak interaction.

\footnotetext{
${ }^{7}$ See equations (A.15, A.18).
} 


\section{Appendix B}

\section{NO $\nu$ A Kalman Tracker}

For track propagation in the Kalman Tracker, each track is represented as a system with two parameters: position and slope. A minimum of two cell hits are required to estimate the parameters. Track seeds are formed from pairs of cell hits separated by as much as 3 planes in their view. The track starting point is assumed to be at the center of the cell hit with the highest $Z$ coordinate, and the track propagation is carried on towards lower $Z$ coordinates. Tracks are propagated plane by plane, using the current estimates of the track position and slope, to estimate the location of the expected track's cell hits in the next plane. For cell hits on the projected plane, a $\Delta \chi^{2}$ test is calculated from the inclusion of the new cell hit in the track. If the $\Delta \chi^{2}$ is less than a default value, the cell hit is added to the track. After a new cell hit is added to the track, a new track fit is done to replace the older position and slope parameters. When the estimated track position for the next cell hit falls in an inactive cell, and no other cell hits in that plane are added to the track, the plane will not count for the propagation algorithm. The track propagation continues until three consecutive planes with no cells hits are found.

When multiple cell hits in a given plane are considered to be part of a track, the addition of each cell hit is determined individually and independently from the inclusion of the other cell hits on the plane. When multiple cell hits are added to a track on one plane, the propagation to the next plane is calculated based on the average of all estimated states 
found from adding each cell hit individually. After each propagation step the resulting tracks are filtered. At the initial propagation, the filter provides the best estimated of the track state for the propagation in the opposite direction. At the final propagation, the filter provides the best estimate of the fit to the track.

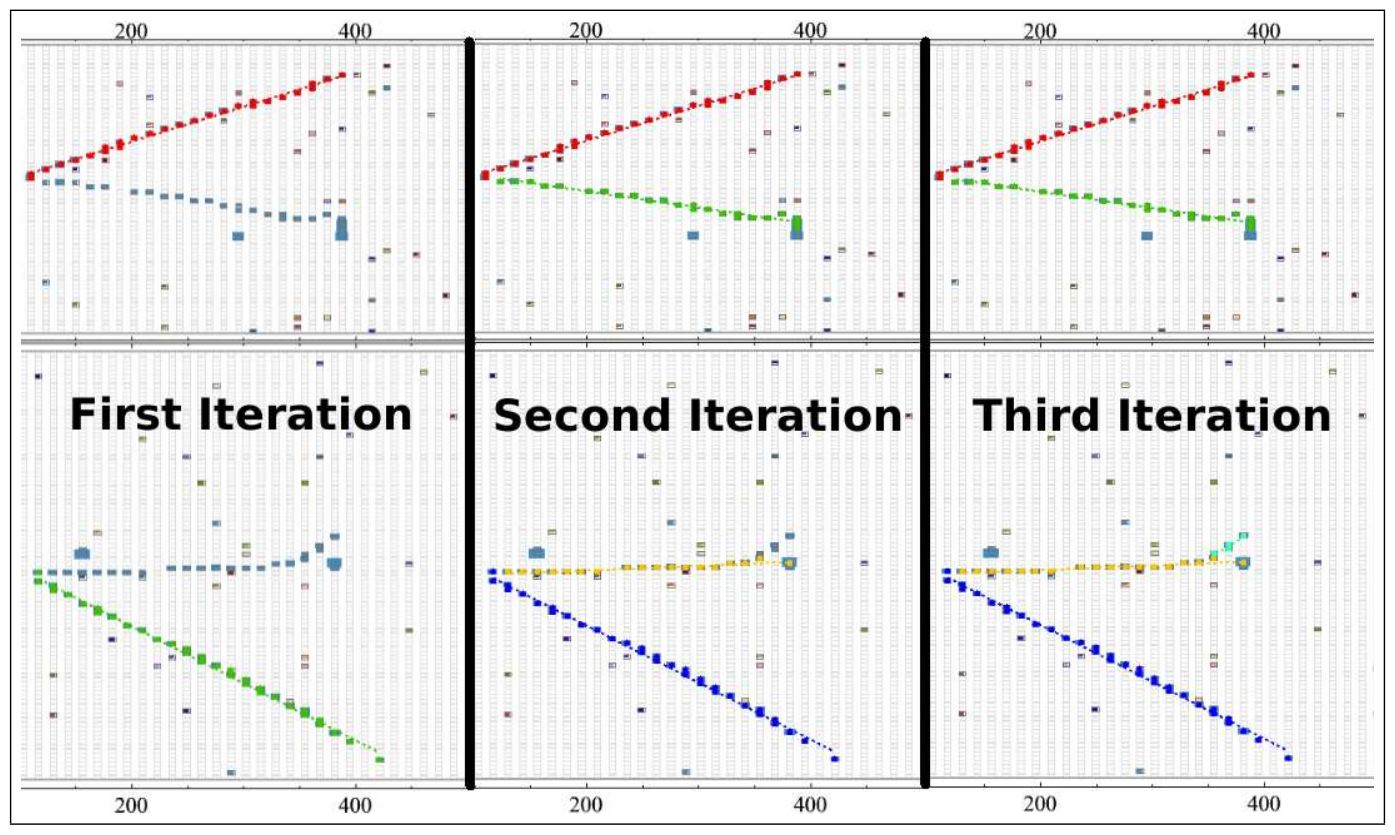

Figure B.1 Sample Event. Illustrates the track finding process. MC simulation.

Before a $2 \mathrm{D}$ track is written in the output the following quality cuts are applied:

- Minimum number of cell hits per track: 4 .

- Minimum number of valid planes crossed by the track: 3 .

- Maximum $\chi^{2}$ per added cell hit: 10.

The cuts reject fake tracks or tracks with poor fits. From the tracks that pass the quality control, the best track found is the one with the lowest $\chi^{2} / N_{\mathrm{ch}}$, where $N_{\mathrm{ch}}$ is the number of cell hits. As mentioned in chapter 4, long muon tracks are expected to undergo multiple scattering at the end of their path. This feature will raise the $\chi^{2} / N_{\mathrm{ch}}$, which is taken into 
account. The best 2D track found is written to the output, and its cell hits are removed from the pool of cell hits in that particular slice. The entire 2D track finding is repeated with the remaining cell hits in the slice to find new tracks.

The algorithm stops when no new tracks pass the cuts, or there are no more available cell hits in the slice. A sample output of the algorithm is presented in figure B.1. From left to right: the first iteration finds one track per view (red and green), the second iteration finds one track per view (green and yellow), and the third iteration finds only one track in the side view (light blue). A flow chart of the algorithm is shown in figure B.2.

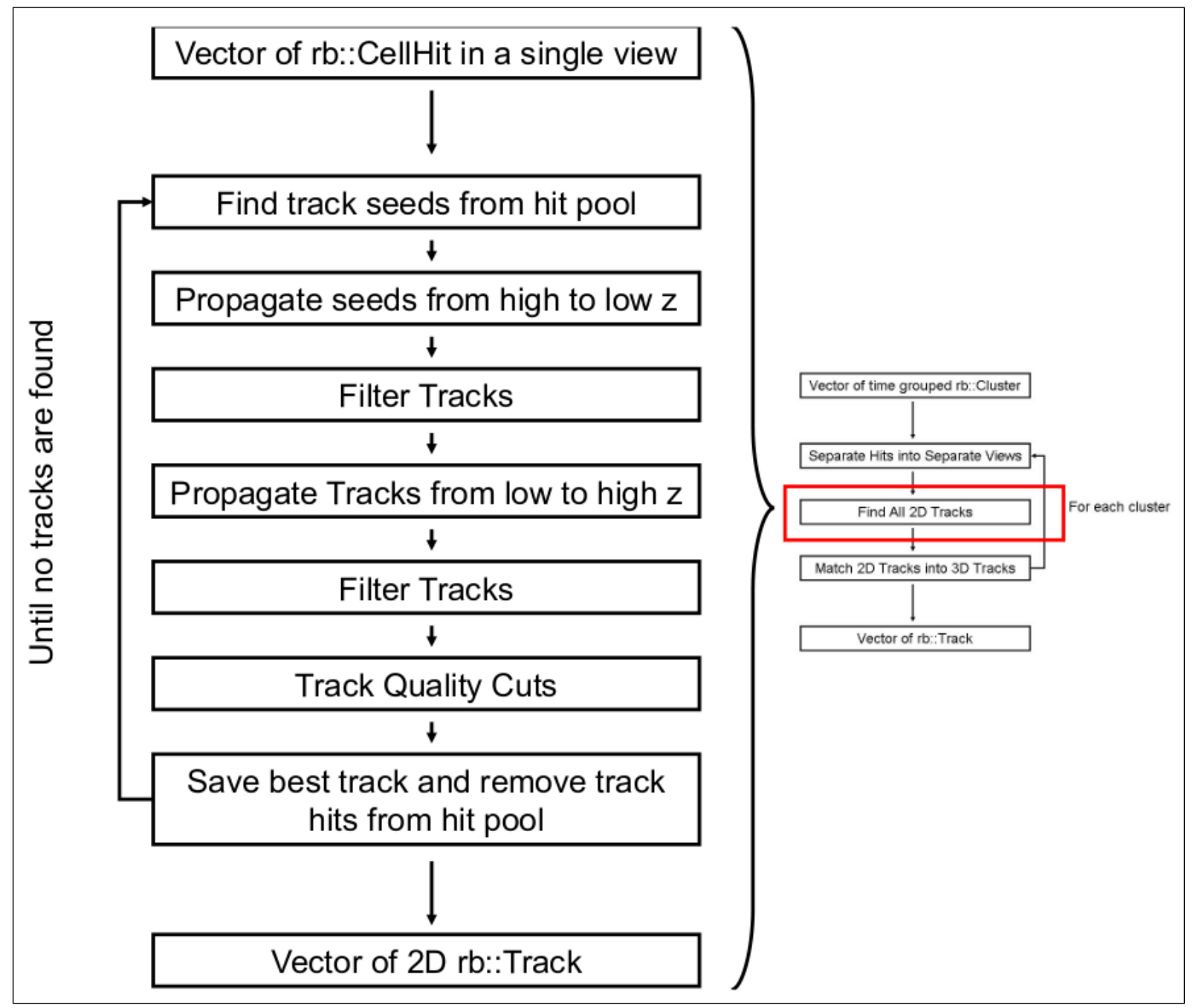

Figure B.2 Flow Chart. Shows how the algorithm finds 2D tracks [140]. 
Within a single slice, 2D tracks from separate views are matched together to form 3D tracks based on a scoring routine. From each pair of $2 \mathrm{D}$ tracks from separate views that overlap in their $Z$ coordinates, a match score is calculated based on the starting and ending $Z$ coordinates of each track:

$$
\text { score }=\frac{\left|Z_{\mathrm{lt}}-Z_{\mathrm{ls}}\right|+\left|Z_{\mathrm{ht}}-Z_{\mathrm{hs}}\right|}{O_{Z}} .
$$

Here $Z_{\mathrm{lt}}$ is the lowest $Z$ coordinated of the $2 \mathrm{D}$ track in the top view, $Z_{\mathrm{ls}}$ is the lowest $Z$ coordinated of the $2 \mathrm{D}$ track in the side view, $Z_{\mathrm{ht}}$ is the highest $Z$ coordinated of the $2 \mathrm{D}$ track in the top view, $Z_{\mathrm{hs}}$ is the highest $Z$ coordinated of the $2 \mathrm{D}$ track in the side view, and $O_{Z}$ is the length of the overlap of the two tracks in the $Z$ coordinate. The two $2 \mathrm{D}$ tracks with the lowest score are matched in each iteration to form a 3D track. The algorithm is repeated until no more 2D tracks overlap in the $Z$ coordinate. Each $2 \mathrm{D}$ track is only allowed to be matched with one $2 \mathrm{D}$ track on the other view. All 3D tracks and unmatched 2D tracks are written to the output. The final output of tracks from the sample event in figure B.1 is presented in figure B.3. Two 3D tracks are reconstructed (red and green), and one 2D track is left unmatched (blue). For a full description of the Kalman Tracker see [140]. 


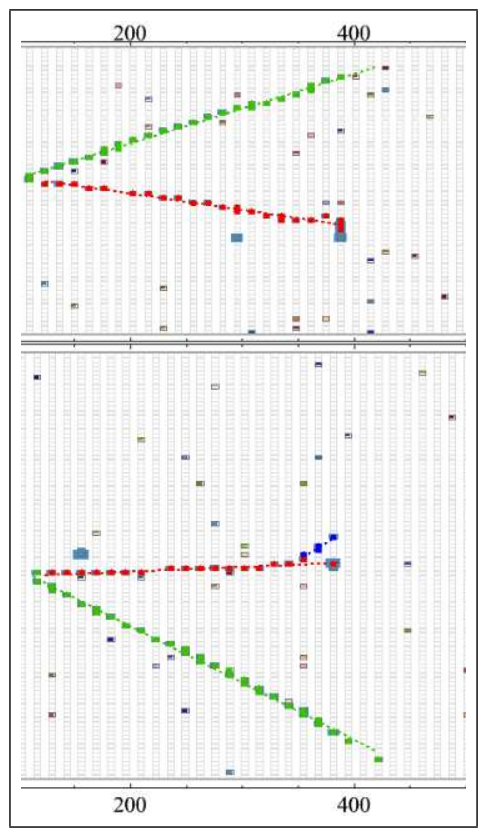

Figure B.3 Sample Event. Final output of tracks from the sample event in figure B.1. 


\section{Appendix C}

\section{Unfolding Algorithm, TSVDUnfold}

One of the many uses of the MC simulated sample is that the reconstructed neutrino energies can be compared to the corresponding true energies. Figure 6.14, for example, is a comparison of true and reconstructed neutrino energies. From this figure it is clear that some events do not have their reconstructed energy in the appropriate energy bin. There are various reason for this to happen, most of them already mentioned. For example figures 6.10a and $6.10 \mathrm{~b}$ show that muons with true energy less than $0.4 \mathrm{GeV}$ attain a reconstructed energy $25 \%$ higher than the true energy. A solution to this issue comes from the use of an unfolding algorithm [141]. This is based on the knowledge of both true and reconstructed energies, which allows to take into account the inefficiencies and resolutions of the detector to try to place the reconstructed events in the appropriate energy bin.

\section{Singular Value Decomposition Unfolding Algorithm}

The unfolding algorithm incorporates the Singular Value Decomposition (SVD) method to find the detector's response matrix. The inputs to the algorithm are [141]:

- $\vec{x}^{\text {ini }}$ Vector of simulated events.

- $\vec{b}^{\text {ini }}$ Vector of reconstructed simulated events. 
The components of the $\vec{x}^{\text {ini }}$ vector are the MC simulated events that realistically represent the underlying physics of the studied situation. The components of the $\vec{b}^{\text {ini }}$ vector are reconstructed $\mathrm{MC}$ simulated events which contain information about the performance of the detector. The goal of the algorithm is to find a probability matrix $\hat{A}$ that will relate the two vectors [141]:

$$
\hat{A} \vec{x}^{\text {ini }}=\vec{b}^{\text {ini }}
$$

Once the matrix is know, the algorithm performs the unfolding procedure that takes the real life measurements gathered in the vector $\vec{b}$ to an unfolded vector $\vec{x}$ that will represent the most probable distribution of events given the performance of the detector [141]:

$$
\hat{A} \vec{x}=\vec{b}
$$

To obtain the vector $\vec{x}$ requires the knowledge of the determinant of $\hat{A}$, which might not be a straightforward endeavor since the components of the matrix could have values ranging from big numbers to very small numbers. The inverses of these numbers could introduce a wide variety of rapidly oscillating solutions to the mathematical problem. The SVD method is a powerful tool that provides useful information about the matrix, e.g. if $\hat{A}$ is orthogonal, all its singular values are 1 . In this method the matrix $\hat{A}$ is represented as the product of three matrices $^{1}[141]$ :

$$
\hat{A}=U S V^{T}
$$

where $U$ and $V$ are orthogonal matrices, and $S$ is a diagonal matrix with non-negative entries called singular values. These represent the reconstruction efficiencies per energy bin.

$$
{ }^{1} V V^{T}=1=V^{T} V, U U^{T}=1=U^{T} U .
$$


As mentioned above, if some of these singular values are small, the system of equations could become very difficult to solve.

With $\vec{b}$ and $\hat{A}$ known, solving for $\vec{x}$ requires the use of the SVD method [141]:

$$
\begin{gathered}
\hat{A} \vec{x}=\vec{b}=U S V^{T} \vec{x}=U S \vec{z}, \\
U^{T} U S \vec{z}=U^{T} \vec{b}=\vec{d}=S \vec{z}, \\
S^{-1} \vec{d}=\vec{z}=V^{T} \vec{x}, \\
\vec{x}=V \vec{z}=V S^{-1} \vec{d}=V S^{-1} U^{T} \vec{b}=\hat{A}^{-1} \vec{b} .
\end{gathered}
$$

The solution means that $\vec{b}$ is a set of orthonormalized functions of a parameter $i=1, \ldots, n_{b}$. The basis of these functions are the columns of the matrix $U$, and the elements of $\vec{d}$ are the coefficients of the decomposition. The vector $\vec{x}$ is decomposed in a series of orthonormalized functions of a parameter $j=1, \ldots, n_{x}$. These functions gather in the columns of the matrix $V$. The coefficients of the decomposition are the components of the vector $\vec{z}$. At the end, the initial problem is reduced to solving a diagonal system:

$$
\vec{z}=S^{-1} \vec{d},
$$

which is just a matter of inverting the diagonal matrix $S$, i.e. inverting the singular values.

If some of the singular values are small, and the measurements in $\vec{b}$ have associated errors, the exact solution does not give any useful information. The following 2 by 2 system 
is presented as an example:

$$
\begin{gathered}
\vec{b}=\left(\begin{array}{c}
b_{1} \\
b_{2}
\end{array}\right), \quad U=V=\frac{1}{\sqrt{2}}\left(\begin{array}{cc}
1 & 1 \\
1 & -1
\end{array}\right), \quad S=\left(\begin{array}{cc}
1 & 0 \\
0 & \epsilon
\end{array}\right), \\
\vec{x}=V S^{-1} U^{T} \vec{b}=\frac{1}{\sqrt{2}}\left(\begin{array}{cc}
1 & 1 \\
1 & -1
\end{array}\right)\left(\begin{array}{cc}
1 & 0 \\
0 & \frac{1}{\epsilon}
\end{array}\right)\left(\begin{array}{cc}
1 & 1 \\
1 & -1
\end{array}\right) \frac{1}{\sqrt{2}} \vec{b} \\
\vec{x}=V S^{-1} U^{T} \vec{b}=\frac{1}{2}\left(\begin{array}{cc}
1 & 1 \\
1 & -1
\end{array}\right)\left(\begin{array}{cc}
1 & 1 \\
\frac{1}{\epsilon} & \frac{-1}{\epsilon}
\end{array}\right) \vec{b}=\frac{1}{2}\left(\begin{array}{cc}
1+\frac{1}{\epsilon} & 1-\frac{1}{\epsilon} \\
1-\frac{1}{\epsilon} & 1+\frac{1}{\epsilon}
\end{array}\right) \vec{b} \\
\vec{x}=\frac{1}{2}\left(\begin{array}{c}
\left(1+\frac{1}{\epsilon}\right) b_{1}+\left(1-\frac{1}{\epsilon}\right) b_{2} \\
\left(1-\frac{1}{\epsilon}\right) b_{1}+\left(1+\frac{1}{\epsilon}\right) b_{2}
\end{array}\right)=\frac{b_{1}+b_{2}}{2}\left(\begin{array}{c}
1 \\
1 \\
1
\end{array}\right)+\frac{b_{1}-b_{2}}{2 \epsilon}\left(\begin{array}{c}
z_{1} \\
-1
\end{array}\right) \\
\vec{x}=\vec{V}_{1}+z_{2} \vec{V}_{2}=\frac{d_{1}}{s_{1}} \vec{V}_{1}+\frac{d_{2}}{s_{2}} \vec{V}_{2} .
\end{gathered}
$$

To determine if the components of the vectors $\vec{V}_{1}$ and $\vec{V}_{2}$ are statistically significant to the problem, the following relation between the coefficients $d_{1}$ and $d_{2}$ needs to be true [141]:

$$
d_{2}^{2} \leq d_{1}
$$

If relation C. 7 is true, the components of $\vec{V}_{2}$ are not statistically significant and $d_{2}$ is simply a random number. However, if:

$$
s_{2}<\frac{1}{\sqrt{d_{2}}}
$$

$\vec{V}_{2}$ dominates over $\vec{V}_{1}$, and the result is meaningless. Since the $s_{i}$ are the efficiencies per energy bin, what this means is that with very low efficiencies, the correct bin for a particular 
event can not be resolved between bin 1 or 2 .

In the general case, for a smooth distribution of measurements, only the first few $d_{i}$ are statistically significant. In a plot of $\log \left|d_{i}\right|$ vs. $i$, the values of $d_{i}$ should exponentially decrease with increasing $i$ until these reach a point of rapid oscillation, therefore the $d_{i}$ are a series of random numbers. Only the $d_{i}$ occurring before the region of rapid oscillation are statistically significant, and useful in the solution of the problem. Once the number of statistically significant $d_{i}$ is established, the system is regularized to avoid possible meaningless results, as mentioned above [141].

To measure the deviations of $\vec{x}$ from $\vec{x}^{\text {ini }}$, a vector $\vec{\omega}$, with components: $\omega_{j}=x_{j} / x_{j}^{\text {ini }}$, is introduced. The product of the $\omega_{j}$ with the columns of the probability matrix $\hat{A}_{i j}$ results in the number of events that were generated in bin $j$ but belong to bin $i$. The result is the matrix $A$, a number-of-events matrix. Rescaling the equations does not change the exact solution. This unfolding problem is an over determined problem and its solution is obtained by means of the least squares approach [141]:

$$
\sum_{i=1}^{n_{b}}\left(\sum_{j=1}^{n_{x}} \hat{A}_{i j} x_{j}-b_{i}\right)^{2}=\min
$$

whenever the errors in the measurements are all equal. Since that is not the case here, i.e. the errors vary from bin to bin, each equation should be weighted by its measured error [141]:

$$
\sum_{i=1}^{n_{b}}\left(\sum_{j=1}^{n_{x}} \frac{\hat{A}_{i j} x_{j}-b_{i}}{\Delta b_{i}}\right)^{2}=\min .
$$

The general equation is:

$$
(\hat{A} \vec{x}-\vec{b})^{T} B^{-1}(\hat{A} \vec{x}-\vec{b})=\min
$$


where $B$ is the covariance matrix of $\vec{b}$, which gathers the errors in the measurements. $B$ is a symmetric and positive-definite matrix, therefore its SVD is:

$$
B=Q R Q^{T}
$$

with:

$$
R_{i i} \equiv r_{i}^{2}>0 \text { and } R_{i j}=0, \text { for } i \neq j .
$$

If the statistics of the MC simulation are one or two orders of magnitude larger than the data statistics, the unfolding error is dominated by the measurement errors, which is the case in the this analysis. Dividing each equation by its measured error gives them equal footing in the problem, and equation (C.11) transforms to [141]:

$$
(A \vec{\omega}-\vec{b})^{T} Q R^{-1} Q^{T}(A \vec{\omega}-\vec{b})=\min ,
$$

with:

$$
\tilde{A}_{i j}=\frac{1}{r_{i}} \sum_{m} Q_{i m} A_{m j}, \quad \tilde{b}_{i}=\frac{1}{r_{i}} \sum_{m} Q_{i m} b_{m}
$$

such that:

$$
(A \vec{\omega}-\vec{b})^{T} Q R^{-1} Q^{T}(A \vec{\omega}-\vec{b})=\min =(\tilde{A} \vec{\omega}-\tilde{b})^{T}(\tilde{A} \vec{\omega}-\tilde{b}) .
$$

Thus,

$$
\sum_{i} \tilde{A}_{i j} \omega_{j}=\tilde{b}_{i}
$$


The regularization is achieved by introducing a stabilization term (see [142], [143], and [144]) into equation (C.11):

$$
(\tilde{A} \vec{\omega}-\tilde{b})^{T}(\tilde{A} \vec{\omega}-\tilde{b})+\tau \cdot(C \vec{\omega})^{T}(C \vec{\omega})=\min ,
$$

where $\tau$ is a stabilization parameter, and $C$ is the a priori condition matrix. These are both problem dependent, and control the equations that present the irregular behavior. The components $C_{i j}$ are chosen such that the components of $\vec{\omega}$ with irregular behavior are suppressed.

The solution to equation (C.18), for any $\tau$, requires the damped least squares approach [144]:

$$
\left[\begin{array}{c}
\tilde{A} C^{-1} \\
\sqrt{\omega} \mathbf{I}
\end{array}\right] C \vec{\omega}=\left[\begin{array}{l}
\tilde{b} \\
\mathbf{0}
\end{array}\right],
$$

from which a similar result as in equation (C.5) is achieved ${ }^{2}$ :

$$
\tilde{d}=\tilde{S}^{-1} S \vec{d}, \quad \tilde{z}=\tilde{S}^{-1} \vec{d}, \quad \vec{\omega}=C^{-1} V \tilde{z}, \quad \tilde{x}_{i}=x_{i}^{\mathrm{ini}} \omega_{i},
$$

where:

$$
\tilde{s}_{i}=\frac{s_{i}^{2}-\tau}{s_{i}},
$$

are the new singular values. The choice of $\tau$ comes from the $\log \left|d_{i}\right|$ vs. $i$ plot. The singular value $\tilde{s}_{k}$ of the last statistically significant equation, $k$, determines it:

$$
\tau=\tilde{s}_{k}^{2} .
$$

\footnotetext{
${ }^{2}$ Detailed calculation in [144], Chapter 25, Section 4.
} 
With all the pieces of the algorithm together, the unfolding procedure is the following:

1. Define the number of bins $n_{b}$, and their boundaries.

2. Define the number of bins $n_{x}$, and their boundaries.

3. Build the condition matrix $C$ introduced in equation (C.18), and calculate its inverse $C^{-1}$

4. Generate the simulated events to find: $\vec{x}^{\text {ini }}$, reconstruct the simulated events to find: $\vec{b}^{\mathrm{ini}}$, and construct $A$, which is the number-of-events matrix introduced in equation (C.14).

5. Calculate the covariance matrix $B$ introduced in equation (C.11).

6. Calculate $\tilde{A}_{i j}$, and $\tilde{b}_{i}$ from equation (C.15).

7. Multiply $\tilde{A} C^{-1}$ to fulfill equation (C.19).

8. Plot $\log \left|d_{i}\right|$ vs. $i$ to determine the last statistically significant equation $k$, and set $\tau$ (see equation (C.22)).

9. Obtain the unfolded distribution of events $\tilde{x}$ from equation (C.20).

ROOT (see $[145,146])$ has a tool, TSVDUnfold, that implements the unfolding algorithm in a very user friendly way. This tool requires the user to provide:

- $\vec{x}^{\text {ini }}$.

- $\vec{b}^{\text {ini }}$.

- $A$. 
With these inputs, TSVDUnfold goes through steps 1 to 8 in the algorithm. Then it asks the user to use the $\log \left|d_{i}\right|$ vs. $i$ plot to provide the parameter $k$. Based on simulated events and reconstructed simulated events, TSVDUnfold calculates the matrices $B$ and $C$. At this point it trained itself to be able to handle real data. Now the user provides a new vector: $\vec{b}^{\text {data }}$, which contains all the real data events. From $\vec{b}^{\text {data }}$ and a set $^{3}$ of toy MC simulated events, generated by TSVDUnfold itself, it calculates a new covariance matrix $B^{\text {data }}$, which is the error matrix for the unfolded distribution. Finally, after training, TSVDUnfold goes through step 9 in the algorithm and gives the unfolded distributions $\tilde{x}$ and $\tilde{x}^{\text {data }} . \tilde{x}$ is the unfolded distribution for the reconstructed simulated events, and $\tilde{x}^{\text {data }}$ is that for the real data events.

\section{Unfolded Distributions}
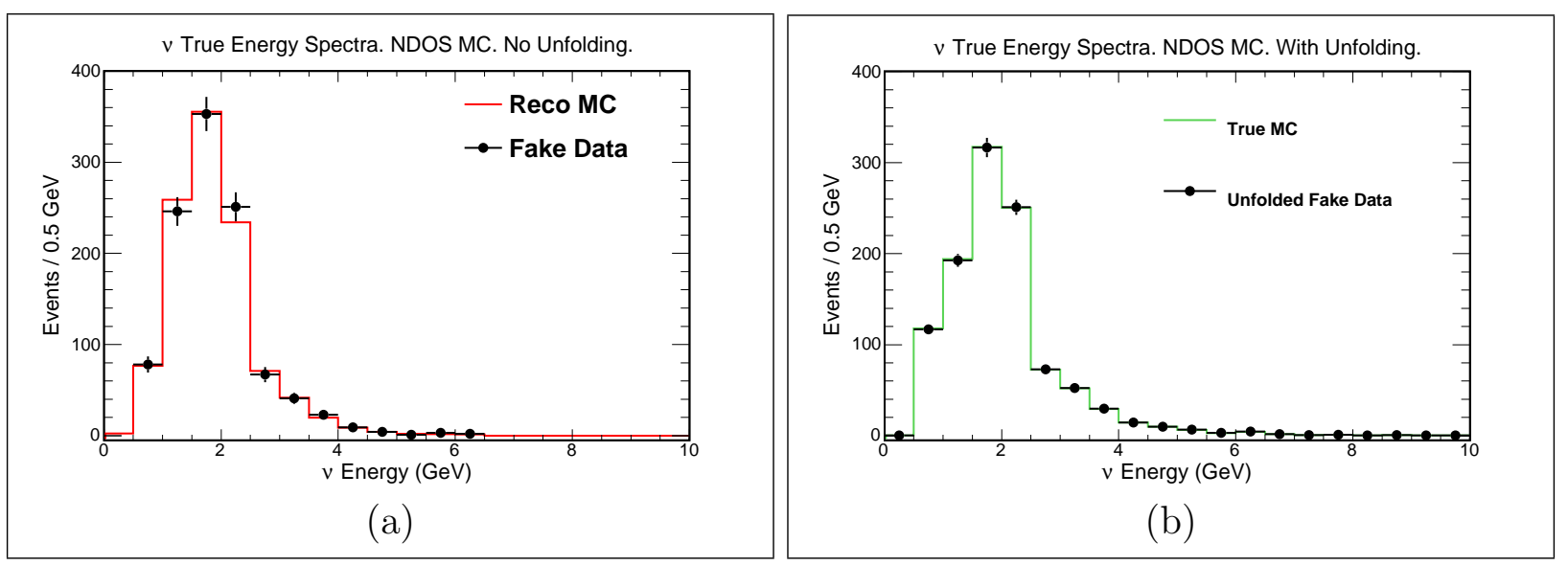

Figure C.1 Neutrino Energy Distributions Before And After Unfolding. (a) Reconstructed MC simulation (red) normalized to fake data (black) area. (b) True MC simulation (green) normalized to unfolded data (black) area. MC simulation.

Following the steps presented at the end of the previous section, and in order to train

\footnotetext{
${ }^{3}$ The size of this toy MC simulated set is entered by the user. The recommended size is $n_{\mathrm{TSVD}}=100$ events.
} 
the algorithm, the MC simulated sample is divided into two sets: one used to build $\vec{x}^{\text {ini }}$, training sample, and the other one ${ }^{4}$ used to build $\vec{b}^{\text {ini }}$, testing sample. The TSVDUnfold is fed with the due inputs, and the results of the training process are presented in figure C.1. As presented in the previous section, the parameter $k$ comes from figure C.2b, which shows that the last statistically significant equation is that with $i=2$, since for higher $i$ the distribution oscillates randomly around $\left|d_{i}\right|=1$. The parameter $k$ is set to:

$$
k=2, \quad \tau=\tilde{s}_{2}^{2}
$$

The migration of events from their reconstructed energy bins to their unfolded energy bins is presented in figure C.2a, which is the covariance matrix $B^{\text {data }}$ mentioned above. The diagonal entries between $1 \mathrm{GeV}$ and $2.5 \mathrm{GeV}$ are $20.1 \%$ of the total entries. In this case the correct bin is reconstructed properly. The off diagonal bins in that same energy region, and colored with light orange, account for $27.2 \%$ of all entries.
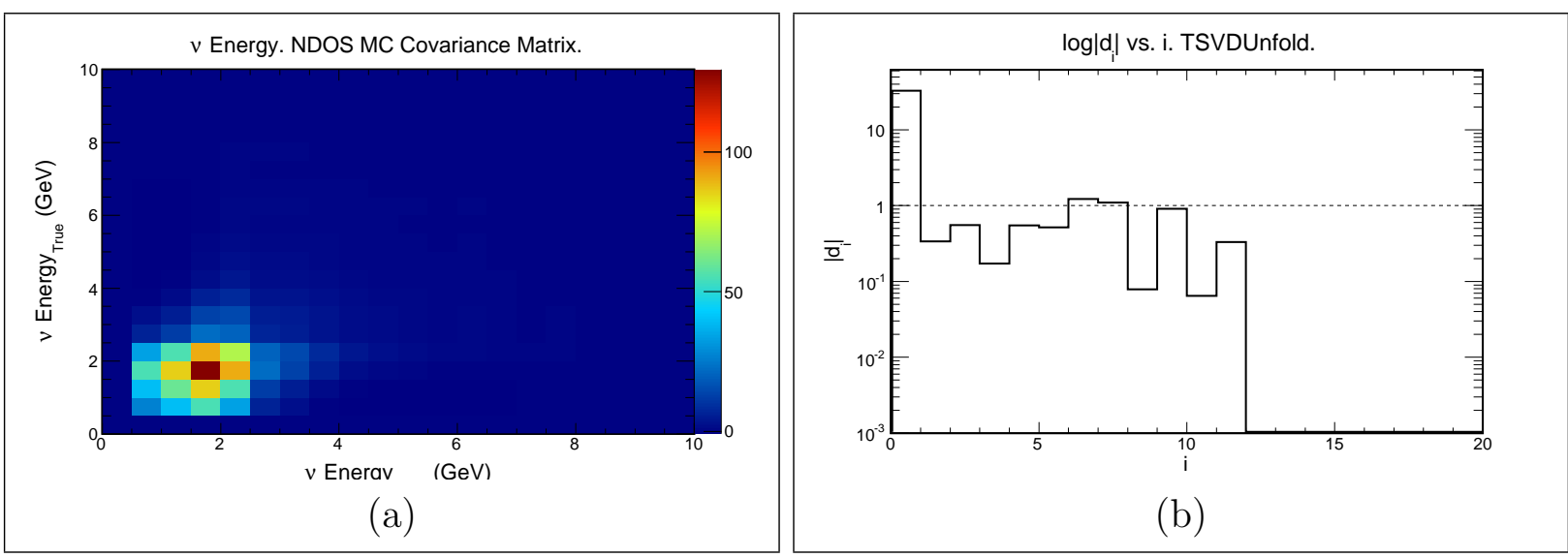

Figure C.2 Results From Unfolding Fake Data. (a) The covariance matrix $B^{\text {data }}$ (b) $\log \left|d_{i}\right|$ vs. $i$. MC simulation.

Figure C.1 shows 4 distributions: The true MC simulated events, the reconstructed MC

${ }^{4}$ About $10 \%$ the size of the first sample. 
simulated events, the fake data ${ }^{5}$ events, and the unfolded events. True MC and Reco MC simulations resemble figure 6.14. The fake data, as part of the MC simulated sample, should have the same shape as the Reco MC simulation does, which is the case in figure C.1a. The unfolded distribution sits on top of the true MC simulation one, which means that the unfolded procedure worked as expected, as seen in figure C.1b. The errors in the unfolded distribution come from the input to the covariance matrix, which in this case are statistical errors in the fake data.

The goal of the unfolding was to place all the fake data events into their most probable energy bins given the performance of the detector embedded in the calculated covariance matrices. This corrects for the discrepancies between reconstructed and simulated events presented in chapter 6 .

The training and testing of the unfolding algorithm are satisfactory, and the algorithm is applied to the NDOS data. In order to find the unfolded data distributions, $\vec{b}^{\text {data }}$ is filled with real NDOS events instead of fake data events, and the covariance matrix $B^{\text {data }}$ is calculated based on the real data measured errors. Everything else is the same as presented above, since it is the result of training the algorithm.

\footnotetext{
${ }^{5}$ The small MC simulated testing sample.
} 


\section{Appendix D}

\section{List Of Muon Neutrino Charged}

\section{Current Candidate Events}

\begin{tabular}{|c|c|c|c|c|c|c|c|c|}
\hline Run & Event & $\begin{array}{c}E_{\nu} \\
(\mathrm{GeV})\end{array}$ & Run & Event & $\begin{array}{c}E_{\nu} \\
(\mathrm{GeV})\end{array}$ & Run & Event & $\begin{array}{c}E_{\nu} \\
(\mathrm{GeV})\end{array}$ \\
\hline 13067_01 & 52339 & 1.1 & 13067_05 & 242768 & 1.3 & 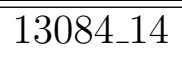 & 636949 & 1.4 \\
\hline 13097_09 & 401527 & 2.0 & 13119_19 & 878982 & 1.4 & 13120_06 & 275972 & 1.5 \\
\hline 13131_08 & 369633 & 2.3 & 13168_09 & 439180 & 2.0 & 13173_00 & 21364 & 1.0 \\
\hline 13178_09 & 423895 & 1.0 & 13209_07 & 351868 & 1.5 & 13210_12 & 558497 & 0.8 \\
\hline 13228_00 & 7822 & 0.9 & 13247_01 & 88704 & 1.0 & 13247_08 & 381991 & 1.3 \\
\hline 13247_10 & 468719 & 1.0 & 13286_00 & 31278 & 0.9 & 13286_04 & 185526 & 1.0 \\
\hline 13289_00 & 3856 & 1.8 & 13352_09 & 455006 & 1.5 & $13352 \_15$ & 686658 & 2.1 \\
\hline 13359_03 & 149922 & 1.3 & 13359_18 & 838462 & 2.1 & 13390_19 & 898936 & 1.2 \\
\hline 13405_00 & 21468 & 2.1 & 13405_06 & 283899 & 2.5 & 13405_08 & 373623 & 2.1 \\
\hline 13405_09 & 423816 & 1.0 & 13406_05 & 269806 & 1.1 & 13406_19 & 895717 & 2.0 \\
\hline 13417_14 & 647470 & 2.0 & $13417 \_40$ & 1815674 & 1.5 & 13485_18 & 898130 & 0.9 \\
\hline 13504_00 & 5587 & 1.6 & 13508_11 & 502607 & 0.9 & 13508_17 & 791080 & 1.4 \\
\hline 13521_07 & 320076 & 1.7 & 13523_09 & 429603 & 2.0 & 13525_00 & 30494 & 3.1 \\
\hline 13526_03 & 173654 & 1.4 & 13528_16 & 704288 & 0.9 & 13531_02 & 100521 & 1.1 \\
\hline 13531_02 & 100521 & 1.1 & 13540_09 & 432385 & 1.0 & 13543_04 & 197375 & 0.9 \\
\hline 13550_01 & 67262 & 1.0 & 13557_20 & 853445 & 3.5 & 13567_00 & 32484 & 1.1 \\
\hline 13648_05 & 224998 & 1.9 & 13659_03 & 154457 & 2.1 & 13659_07 & 333945 & 1.2 \\
\hline 13659_12 & 576285 & 1.2 & 13675_05 & 251706 & 1.7 & 13679_08 & 402161 & 1.9 \\
\hline 13679_12 & 565281 & 1.8 & 13679_13 & 624373 & 1.1 & 13709_18 & 838184 & 0.9 \\
\hline 13730_20 & 948244 & 1.0 & 13731_18 & 858628 & 1.4 & 13732_01 & 83757 & 1.5 \\
\hline 13732_04 & 224730 & 1.4 & 13732_05 & 233639 & 1.0 & 13732_06 & 281760 & 3.1 \\
\hline 13737_02 & 112479 & 1.2 & 13737_04 & 188755 & 0.9 & 13737_08 & 413309 & 1.0 \\
\hline 13743_03 & 176473 & 1.0 & 13754_01 & 62591 & 1.0 & 13754_02 & 123870 & 2.5 \\
\hline 13756_04 & 219066 & 3.3 & 13759_05 & 248715 & 1.2 & 13763_04 & 162894 & 0.6 \\
\hline 13767_01 & 68409 & 1.2 & 13767_02 & 81374 & 3.4 & - & - & - \\
\hline
\end{tabular}

Table D.1 Selected QE Candidate Events. 
All the selected QE neutrino candidates before cosmic background subtraction are presented in table D.1. All the selected non-QE neutrino candidates before cosmic background subtraction are presented in tables D.2 and D.3.

\begin{tabular}{|c|c|c|c|c|c|c|c|c|}
\hline Run & Event & $\begin{array}{c}E_{\nu} \\
(\mathrm{GeV})\end{array}$ & Run & Event & $\begin{array}{c}E_{\nu} \\
(\mathrm{GeV})\end{array}$ & Run & Event & $\begin{array}{c}E_{\nu} \\
(\mathrm{GeV})\end{array}$ \\
\hline "13068_01 & 70583 & $\bar{~} 1.9$ & "13068_02 & 133375 & $\bar{~} 1.1$ & "13068_03 & 183770 & 1.8 \\
\hline 13071_05 & 273089 & 1.5 & 13075_15 & 704141 & 2.0 & 13075_17 & 781439 & 2.5 \\
\hline 13084_01 & 87787 & 1.6 & 13087_01 & 57985 & 3.2 & 13094_05 & 265850 & 2.0 \\
\hline 13097_11 & 516437 & 2.4 & 13097_11 & 517102 & 1.8 & 13097_13 & 604309 & 2.0 \\
\hline 13117_03 & 151666 & 0.9 & 13120_00 & 38220 & 1.3 & 13123_04 & 158468 & 1.3 \\
\hline 13131_01 & 73464 & 2.4 & 13160_01 & 61236 & 1.3 & 13160_02 & 112807 & 2.0 \\
\hline 13160_11 & 512920 & 1.5 & 13160_15 & 687140 & 2.3 & 13166_09 & 410288 & 1.3 \\
\hline 13173_12 & 576650 & 1.6 & 13176_04 & 244242 & 1.7 & 13176_11 & 539526 & 1.4 \\
\hline 13178_04 & 206510 & 1.6 & 13178_05 & 266544 & 2.1 & 13178_07 & 323898 & 4.5 \\
\hline 13189_02 & 95469 & 1.5 & 13190_00 & 7437 & 2.4 & 13208_03 & 140024 & 1.9 \\
\hline 13209_01 & 71489 & 1.5 & 13209_14 & 655627 & 2.0 & 13209_19 & 868527 & 0.8 \\
\hline 13210_13 & 608565 & 3.1 & 13213_02 & 94255 & 1.8 & 13214_03 & 177158 & 1.4 \\
\hline 13220_01 & 84251 & 2.2 & 13236_05 & 246984 & 2.4 & 13241_11 & 541964 & 1.1 \\
\hline 13247_01 & 55167 & 1.6 & 13247_09 & 430374 & 1.8 & 13262_01 & 55638 & 4.0 \\
\hline 13262_01 & 70110 & 1.6 & 13265_04 & 229559 & 2.6 & 13265_06 & 292238 & 1.3 \\
\hline 13277_01 & 79372 & 1.9 & 13286_01 & 48964 & 2.3 & 13286_02 & 120280 & 1.5 \\
\hline 13312_06 & 315859 & 1.7 & 13320_15 & 685032 & 1.6 & 13330_02 & 117981 & 1.9 \\
\hline 13333_06 & 285527 & 1.9 & 13333_10 & 488169 & 1.3 & 13333_12 & 546198 & 2.7 \\
\hline 13333_15 & 694421 & 3.0 & 13339_00 & 20224 & 2.2 & 13339_03 & 135737 & 1.9 \\
\hline 13348_06 & 271122 & 1.5 & 13352_05 & 269563 & 1.7 & 13352_10 & 494823 & 2.0 \\
\hline 13352_15 & 706818 & 1.2 & 13353_13 & 611234 & 1.1 & 13359_12 & 558228 & 1.7 \\
\hline 13360_05 & 260305 & 2.0 & 13365_02 & 118904 & 1.7 & 13365_03 & 141066 & 1.3 \\
\hline 13389_02 & 98800 & 2.9 & 13389_11 & 533620 & 2.8 & 13393_07 & 326670 & 1.6 \\
\hline 13393_19 & 880031 & 2.3 & 13393_20 & 933931 & 1.6 & 13399_06 & 300556 & 2.0 \\
\hline 13399_10 & 470075 & 1.7 & 13399_12 & 578886 & 1.5 & 13399_12 & 589675 & 1.4 \\
\hline 13405_06 & 281676 & 2.3 & 13405_18 & 857049 & 3.0 & 13405_20 & 936462 & 2.1 \\
\hline 13406_08 & 396159 & 2.6 & 13406_13 & 641569 & 1.1 & 13406_20 & 945997 & 1.5 \\
\hline 13407_00 & 40739 & 1.9 & 13407_09 & 435601 & 2.1 & 13409_14 & 668614 & 1.8 \\
\hline 13441_06 & 320584 & 1.4 & 13443_09 & 398666 & 1.9 & 13443_14 & 664933 & 1.3 \\
\hline
\end{tabular}

Table D.2 Selected Non-QE Candidate Events, I. 


\begin{tabular}{|c|c|c|c|c|c|c|c|c|}
\hline Run & Event & $\begin{array}{c}E_{\nu} \\
(\mathrm{GeV})\end{array}$ & Run & Event & $\begin{array}{c}E_{\nu} \\
(\mathrm{GeV})\end{array}$ & Run & Event & $\begin{array}{c}E_{\nu} \\
(\mathrm{GeV})\end{array}$ \\
\hline "13444_09 & 445873 & 2.0 & 13444_13 & 632045 & 1.3 & 13485_14 & 680500 & 1.5 \\
\hline 13490_00 & 27697 & 2.2 & 13490_00 & 43841 & 1.9 & 13495_15 & 714932 & 2.2 \\
\hline 13504_03 & 163755 & 2.9 & 13504_03 & 170439 & 2.8 & 13505_06 & 317921 & 2.4 \\
\hline 13508_00 & 31211 & 3.4 & 13508_12 & 560534 & 1.7 & 13508_13 & 610384 & 2.7 \\
\hline 13512_06 & 292369 & 2.2 & 13512_14 & 667424 & 2.4 & 13516_01 & 55536 & 1.5 \\
\hline 13521_04 & 219941 & 1.3 & 13521_07 & 334779 & 1.7 & 13523_03 & 153709 & 3.7 \\
\hline 13523_12 & 573584 & 1.7 & 13523_22 & 1005777 & 1.8 & 13525_06 & 275923 & 1.1 \\
\hline 13531_08 & 373797 & 1.3 & 13533_07 & 337448 & 3.3 & 13533_17 & 806866 & 1.6 \\
\hline 13534_00 & 18678 & 1.3 & 13539_03 & 169453 & 2.3 & 13540_20 & 917878 & 1.6 \\
\hline 13557_07 & 261561 & 2.4 & 13557_14 & 619026 & 2.1 & 13557_15 & 669256 & 1.8 \\
\hline 13611_04 & 184605 & 1.2 & 13619_04 & 205187 & 1.1 & 13619_11 & 525351 & 1.5 \\
\hline 13619_18 & 843304 & 1.2 & 13659_11 & 534603 & 1.9 & 13660_05 & 239565 & 1.5 \\
\hline 13660_07 & 338739 & 1.6 & 13660_10 & 493358 & 2.3 & 13709_16 & 746592 & 1.8 \\
\hline 13710_18 & 864719 & 2.2 & 13711_03 & 150155 & 1.5 & 13711_06 & 300659 & 4.1 \\
\hline 13717_12 & 589572 & 2.3 & 13717_15 & 717932 & 1.3 & 13727_13 & 608348 & 3.3 \\
\hline 13731_14 & 688158 & 2.0 & 13731_19 & 917338 & 1.4 & 13732_03 & 151136 & 1.7 \\
\hline 13732_05 & 236320 & 1.3 & 13737_04 & 197897 & 1.4 & 13737_05 & 263039 & 1.5 \\
\hline 13740_00 & 11613 & 3.0 & 13740_21 & 972374 & 1.8 & $13742 \_05$ & 270585 & 2.0 \\
\hline 13743_00 & 7658 & 1.0 & 13743_08 & 406626 & 1.2 & 13743_23 & 1069794 & 1.8 \\
\hline 13744_04 & 188689 & 1.2 & 13744_05 & 272594 & 2.6 & 13744_07 & 362545 & 2.0 \\
\hline $13744 \_10$ & 495266 & 1.4 & 13746_23 & 1096829 & 2.6 & 13751_15 & 700296 & 1.5 \\
\hline 13754_01 & 85788 & 1.2 & 13754_12 & 588024 & 2.8 & 13754_15 & 691009 & 1.4 \\
\hline 13754_16 & 773307 & 1.8 & 13754_16 & 821744 & 1.5 & 13759_19 & 888335 & 0.9 \\
\hline 13760_17 & 750963 & 2.2 & 13763_01 & 70828 & 1.9 & 13763_09 & 358811 & 1.0 \\
\hline 13764_00 & 4051 & 1.1 & 13765_03 & 146213 & 1.5 & 13767_02 & 91379 & 1.6 \\
\hline 13770_01 & 43260 & 2.0 & 13770_09 & 344492 & 2.3 & 13778_22 & 831025 & 1.4 \\
\hline
\end{tabular}

Table D.3 Selected Non-QE Candidate Events, II. 


\section{BIBLIOGRAPHY}




\section{BIBLIOGRAPHY}

[1] S. Ando, K. Sato, T. Totani, Astropart. Phys. $18,307$.

[2] P. J. E. Peebles. Astrophys. J. 146, 542.

[3] C. Hayashi, Prog. Theor. Phys. 5, 224.

[4] W. Pauli, Open letter to the group of radioactive people at the Gauverien meeting in Tübingen, 1930.

[5] C. L. Cowan, Jr., F. Reines, F. B. Harrison, E. C. Anderson, F. N. Hayes, Phys. Rev. 90, 493.

[6] G. Danvy, J. M. Gaillard, K. Goulianos, L. M. Lederman, N. Mistry, M. Schwartz, J. Steinberger, Phys. Rev. Lett. 9, 36.

[7] [http://www.bnl.gov/bnlweb/history/nobel/nobel_88.asp].

[8] B. J. Bjorken, S. L. Glashow, Phys. Lett. 11 (3), 255.

[9] J. Aubert et al., Phys. Rev. Lett. 33 (23), 1404.

[10] J. Augustin et al., Phys. Rev. Lett. 33 (23), 1406.

[11] S. W. Herb et al., Phys. Rev. Lett. 39 (5), 252.

[12] M. L. Perl et al., Phys. Rev. Lett. 35 (22), 1489.

[13] K. Kodama et al., Phys. Lett. B 504 (3), 218.

[14] B. Pontecorvo, Zh. Eksp. Teor. Fiz 33, 549.

[15] J. N. Bahcall, Sci. Am. 221 (1), 28.

[16] Y. Fukuda et al., Phys. Rev. Lett. 81, 1562. 
[17] Q. R. Ahmad et al., Phys. Rev. Lett. 87 (7), 071301.

[18] Y. Abe et al., Phys. Rev. Lett. 108, 131801.

[19] F. P. An et al., Phys. Rev. Lett. 108, 171803.

[20] J. K. Ahn et al.. Phys. Rev. Lett. 108, 191802.

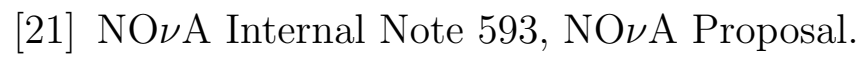

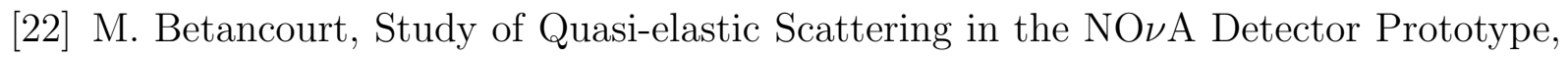
Ph.D. Thesis (2013).

[23] S. L. Glashow, Nucl. Phys. 22, 579.

[24] S. Weinberg, Phys. Rev. Lett. 19, 1264.

[25] A. Salam, Elementary Particle Theory: Relativistic Groups and Analyticity (Nobel Symposium No. 8). N. Svarttholm, Almqvist and Wiksell, Stockholm, 1968.

[26] E. Fermi, Z. Phys. 88, 161.

[27] E. A. Paschos, Electroweak Theory. Cambridge University Press, 2007.

[28] N. Cabibbo, Phys. Rev. Lett 10, 531.

[29] M. Kobayashi, T. Maskawa, Prog. Theor. Phys. 49, 652.

[30] Z. Maki, M. Nakagawa, and S. Sakata, Prog. Theor. Phys. 28, 870.

[31] B. Pontecorvo, Zh. Eksp. Teor. Fiz 33, 1717.

[32] A. A. Aguilar-Arevalo et al., Phys. Rev. D 81, 092005.

[33] S. J. Barish et al., Phys. Rev. D 16, 3103.

[34] D. Allasia et al., Nucl. Phys. B 343, 285.

[35] N. J. Baker et al., Phys. Rev. D 23, 2499. 
[36] G. Fanourakis et al., Phys. Rev. D 21, 562.

[37] T. Kitagaki et al., Phys. Rev. D 28, 436.

[38] S. Bonetti et al., Nuovo Cimento A 38, 260.

[39] N. Armenise et al., Nucl. Phys. B 152, 365.

[40] L. B. Auerbach et al., Phys. Rev. C 66, 015501.

[41] V. Lyubushkin et al., Eur. Phys. J. C 63, 355.

[42] S. V. Belikov et al., Z. Phys. A 320, 625.

[43] J. Brunner et al., Z. Phys. C 45, 551.

[44] [http://pdg.lbl.gov/2014/reviews/rpp2014-rev-nu-cross-sections.pdf], figures 49.1 and 49.2 .

[45] D. Rein, L. M. Sehgal, Nucl. Phys. B 223, 29.

[46] R. P. Feynman, M. Kislinger, F. Ravndal, Phys. Rev. D 3, 2706.

[47] H. M. Gallagher, M. C. Goodman, NuMI-112. PDK-626. Nov. 101995.

[48] J. A. Formaggio, G. P. Zeller, Rev. Mod. Phys. 84, 1307.

[49] A. J. Buras, Rev. Mod. Phys. 52, 199.

[50] R. M. Barnett, Phys. Rev. D 14, 70.

[51] I. Schienbein et al., J. Phys. G 35, 053101.

[52] A. Sirlin, W. Marciano, Nucl. Phys. B 189, 442.

[53] S. Moch, J. Vermaseren, Nucl. Phys. B 573, 853.

[54] C. H. Albright, C. Jarlskog, Nucl. Phys. B 84, 467. 
[55] N. J. Baker et al., Phys. Rev. D 25, 617.

[56] Y. Nakajima et al., Phys. Rev. D 83, 012005.

[57] Y. Hayato, Acta Phys. Polon. B 40, 2477.

[58] D. Casper, Nucl. Phys. Proc. Suppl. 112, 161.

[59] C. A. Bertulani, C. de Conti, Phys. Rev. C 81, 064603.

[60] F. J. Hasert et al., Phys. Lett. B 46, 121.

[61] A. A. Aguilar-Arevalo et al., Phys. Rev. D 82, 092005.

[62] C. H. Lewellyn-Smith, Nucl. Phys. B 228, 205.

[63] [https : //cds.cern.ch/record/161219/files/CM - P00060026.pdf].

[64] M. Fukugita, T. Yanagida, Physics of Neutrinos and Applications to Astrophysics. Springer, 2003. ISBN 3-540-43800-9.

[65] R. N. Mohapatra, G. Senjanovic, Phys. Rev. Lett. 44 (14), 912.

[66] B. Kayser, arXiv:hep-ph/0211134, Neutrino Mass, Mixing, and Flavor Change, 2008.

[67] R. Arnold et al., Phys. Rev. D 89, 111101(R).

[68] S. Davidson, E. Nardi, Y. Nir, arXiv:hep-ph/0802.2962, Leptogenesis, 2008.

[69] Y. Katayama, K. Matumoto, S. Tanaka, E. Yamada, Prog. Theor. Phys. 28, 675.

[70] M. C. Gonzalez-Garcia et al., arXiv:hep-ph/1209.3023, Global fit to the three neutrino mixing: critical look at present precision.

[71] [http://www - numi.fnal.gov/numwork/tdh/TDH_V2_1_Introduction.pdf].

[72] [http://www-nova.fnal.gov/nova_beam_anu.html].

[73] [http://www-fmi.fnal.gov/Preform\%20Goals/Chapter5.pdf]. 
[74] J. J. Evans, arXiv:hep-ph/1307.0721, The MINOS experiment: results and prospects, 2013.

[75] [http : //www - numi.fnal.gov/numwork/tdh/TDH_V2_2_Overview.pdf].

[76] [http://www-bdnew.fnal.gov/operations/accgloss/gloss.html].

[77] [http : //pdg.lbl.gov/2013/listings/rpp2013 - list - K - plus - minus.pdf].

[78] [http: //pdg.lbl.gov/2013/listings/rpp2013 - list - pi-plus - minus.pdf].

[79] P. Adamson et al., Phys. Rev. D 82, 051102.

[80] H. Minakata, H. Nunokawa, JHEP 0110, 001.

[81] [http://www - nova.fnal.gov/plots_and_figures/plot_and_figures.html\#1300_ Theta13_Mass_Hierarchy_CP_phase].

[82] A. J. Norman, NO $\nu$ A Internal Note 11398, Plenary talk for the Neutrino 2014 conference.

[83] R. Talaga, NO $\nu$ A Internal Note 5829, PVC Report.

[84] NO $\nu$ A Internal Note 6075, NDOS photographs by R. Hahn.

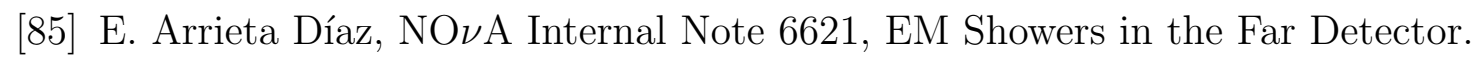

[86] S. Mufson, NO $\nu$ A Internal Note 3865, WBS 2.2 Plenary Liquid Scintillator.

[87] [http://pdg.lbl.gov/2009/reviews/rpp2009-rev-passage-particles-matter.pdf].

[88] E. Arrieta Díaz et al., NO $\nu$ A Internal Note 5187, Wavelength Shifting Fiber Quality Assurance for the $\mathrm{NO} \nu \mathrm{A}$ Experiment.

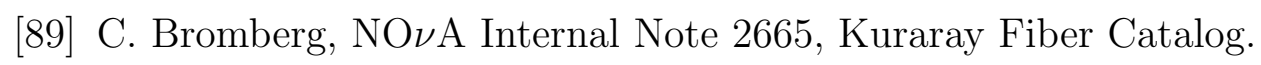

[90] C. Backhouse and P. Shanahan, private communication.

[91] A. J. Norman, J. Phys.: Conf. Ser. 396, 012035. 
[92] S. Kasahara, $\mathrm{NO} \nu \mathrm{A}$ Internal Note 6455, $\mathrm{NO} \nu \mathrm{A} \mathrm{DAQ}$.

[93] C. Backhouse, $\mathrm{NO} \nu \mathrm{A}$ Internal Note 7410, Calibration of the $\mathrm{NO} \nu \mathrm{A}$ Detectors.

[94] E. Arrieta Díaz, NO $\nu$ A Internal Note 7599, Track Multiplicity in NDOS.

[95] T. Kutnink, Detector Response Calibration for the NO $\nu$ A Quasi-elastic Cross Section measurement, M. Sc. Thesis (2012).

[96] A. Fassò et al., The Physics Models of FLUKA: Status and Recent Developments, Proc. CHEP03, La Jolla (2003).

[97] S. Agostinelli et al., Nucl. Instr. and Meth. in Phys. Res. A 506, 250.

[98] A. I. Himmel, NO $\nu$ A Internal Note 5388, The NuMI beam Simulation with Flugg.

[99] C. Andreopoulos et al., The GENIE Neutrino Monte Carlo Generator, Nucl. Instr. and Meth. A 614, 87.

[100] S. Lein, NO $\nu$ A Internal Note 6805, Slicer Overview and Status.

[101] E. Arrieta Díaz, NO $\nu$ A Internal Note 7189, Trackers' Performance.

[102] R. E. Kalman, J. Basic Eng. 81, 35.

[103] E. Arrieta Dìaz, NO $\nu$ A Internal Note 9144, Muon Energy Estimation in NDOS Run II.

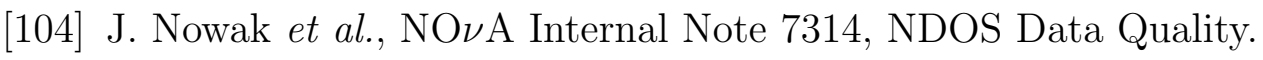

[105] [http ://www - nova.fnal.gov/plots_and_figures/plot_and_figures.html].

[106] R. Hatcher, $\mathrm{NO} \nu \mathrm{A}$ Internal Note 6636, On NO $\nu$ A Flux-Detector Coordinate Transformations.

[107] S. Magill, $\mathrm{NO} \nu \mathrm{A}$ Internal Note 7801, The $\mathrm{NO} \nu \mathrm{A}$ Neutrino Calorimeter.

[108] E. Arrieta Díaz, NO $\nu$ A Internal Note 8824, Muon Energy Estimation in NDOS Run II. 
[109] [http://genie.hepforge.org/manuals/

GENIE_PhysicsAndUserManual_20130615.pdf], figure 2.1.

[110] P. Adamson et al., Phys. Rev. D 77, 072002.

[111] A. V. Lebedev, Ratio of Pion Kaon Production in Proton Carbon Interactions, Ph.D. Thesis (2007).

[112] [http://www - cdf.fnal.gov/physics/statistics/notes/ cdf 1285_KS_test_after_fit.pdf].

[113] M. R. Salzgeber, Measurement of the Inclusive $\nu_{\mu}$ Charged Current Cross Section in the Near Detector of the T2K Experiment, Ph.D. Thesis (2012).

[114] K. Kephart, NO $\nu$ A Internal Note 1136, Near Detector Parameters.

[115] R. Talaga, Private documents and private communication.

[116] [https://cdcvs.fnal.gov/redmine/projects/novaart/repository/entry/trunk/ Geometry/gdml/ndos - $\left.2 x 3-4 b l o c k \_n o m c-x t r u-v a c u u m . g d m l\right]$.

[117] D. MacFarlane et al., Z. Phys. C 26, 1.

[118] J. P. Berge et al., Z. Phys. C 35, 443.

[119] S. Ciampolillo et al., Phys. Lett. B 84, 281.

[120] D. C. Colley et al., Z. Phys. C 2, 187.

[121] P. Bosetti et al., Phys. Lett. B 110, 167.

[122] A. I. Mukhin et al., Sov. J. Nucl. Phys. 30, 528.

[123] D. S. Baranov et al., Phys. Lett. B 81, 255.

[124] S. J. Barish et al., Phys. Rev. D 19, 2521.

[125] T. Eichten et al., Phys. Lett. B 46, 274. 
[126] P. Adamson et al., Phys. Rev. Lett. 102, 211801.

[127] W. J. Marciano, A. I. Sanda, Phys. Lett. B 67, 303.

[128] B. W. Lee, R. E. Shrock, Phys. Rev. D 16, 1444.

[129] P. B. Pal, L. Wolfenstein, Phys. Rev. D 25, 766.

[130] C. Quigg, Gauge Theories of the Strong, Weak, and Electromagnetic Interactions. The Benjamin/Cummings Publishing Company, 1983. ISBN 0-8053-6020-4.

[131] M. Gell-Mann, Nuovo Cimento 4 (S2), 848.

[132] T. Nakano, N. Nishijima, Prog. Theor. Phys. 10 (5), 581.

[133] J. D. Bjorken, S. Drell, Relativistic Quantum Fields. McGraw-Hill, 1965. ISBN 0-07005494-0.

[134] G. 't Hooft, Nucl. Phys. B 33, 173.

[135] [http : //dorigo.wordpress.com/2007/11/10/the-goldstone-theorem-for-realdummies/].

[136] P. Langacker, arXiv:hep-ph/0901.0241, Introduction to the Standard Model and Electroweak Physics, 2009.

[137] S. R. Coleman, E. Weinberg, Phys. Rev. D 7, 1888.

[138] D. F. Griffiths, Introduction to Elementary Particles. WILEY-VCH Verlag GmbH and Co, 2008. ISBN 978-3-527-40601-2.

[139] P. W. Higgs, Phys. Rev. Lett. 13 (16), 508.

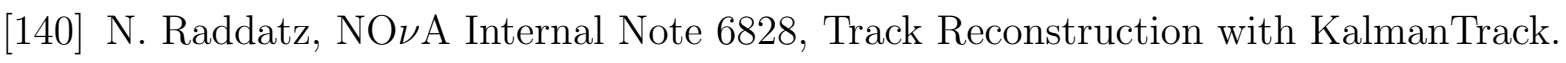

[141] A. Hoecker, V. Kartvelishvili, Nucl. Instrum. Meth. A 732, 469.

[142] V. Blobel, Unfolding methods in high-energy physics experiment, DESY 84-118 (1984). 
[143] M. Schmelling, Nucl. Instr. and Meth. in Phys. Res. A 340, 400.

[144] C. E. Lawson, R. J. Hanson, Solving Least Square Problems, Prentice-Hall Inc., Englewood Cliffs, 1974.

[145] R. Brun, F. Rademakers, ROOT - An Object Oriented Data Analysis Framework, Proc. AIHENP'96 Workshop, Lausanne, Sep. 1996, Nucl. Instr. and Meth. in Phys. Res. A 389, 81.

[146] [http://root.cern.ch/root/html/TSVDunfold.html]. 